TE WHARE WĀNANGA O TE ŪPOKO O TE IKA A MĀUI

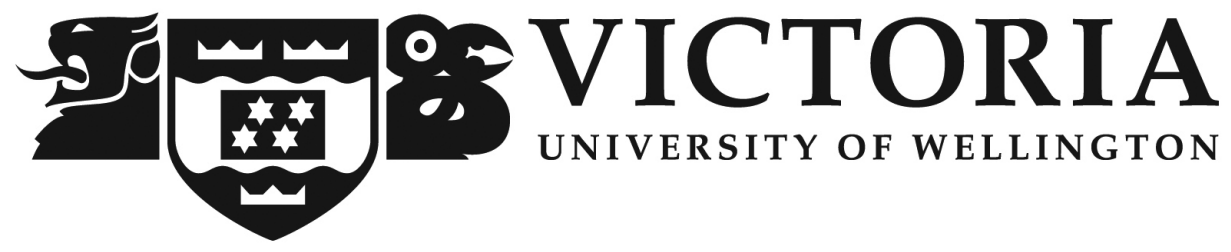

\title{
Diffuse Extragalactic Emission at Low Frequencies within the Epoch of Reionization 0-hour Field \\ MSc Thesis
}

\section{Stefan William Duchesne}

\author{
Supervisor:
}

Assoc. Prof. Melanie Johnston-Hollitt

A thesis submitted to the Victoria University of Wellington in fulfilment of the requirements for the degree of Master of Science in Physics. Victoria University of Wellington 2017 



\begin{abstract}
Low-frequency radio imaging of the southern sky has become available with the advent of the Murchison Widefield Array (MWA). The topic of this thesis is the study of extended, low-frequency radio emission, with a primary focus on the non-thermal synchrotron emission associated with the intra-cluster medium (ICM) of galaxy clusters. We do not limit the study to such emission, however, and investigate a small sample of other interesting and extended radio emission from objects in the southern sky.
\end{abstract}

A significant portion of this work is invested in detecting, and characterising, extended, diffuse radio emission from galaxy clusters within a 45 degree by 45 degree region of the southern sky centred on R.A. $=0^{\circ}$, decl. $=-27^{\circ}$. This field is chosen as a deep MWA image has been made available which is sensitive to extended structures. Within the field we search for low-frequency, diffuse cluster emission, previously detected or otherwise. In doing so we find 34 diffuse radio sources, 3 of which are newly detected haloes, 1 newly detected relic with many new candidates of each. Further, we detect a new phoenix candidate as well as 2 candidate dead radio galaxies at the centre of clusters. We confirm previous observations of such emission as well, and measure properties such as their integrated flux densities, spectral indices, and sizes where possible. We compare our sample of haloes with previously detected haloes and revisit established scaling relations of the radio halo power $\left(P_{1.4}\right)$ with the cluster X-ray luminosity $\left(L_{\mathrm{X}}\right)$ and mass $\left(M_{\mathrm{YZ}, 500}\right)$. We find that both scaling relations are consistent with previous findings despite the increase in sample size, though note that the raw scatter in the data for best-fitting parameters increases with increase in sample size. In this, we demonstrate the utility of low-frequency radio telescopes like the MWA in detecting such emission, showing that the MWA is pushing into higher-redshift, lower-mass systems, though we caution that the low resolution of the MWA can work against us.

We follow-up on two galaxy clusters found to host extended emission-Abell S1136 and Abell S1063. In the case of Abell S1136 we observe the emission at its centre with the Australia Telescope Compact Array (ATCA) and determine the presence of a core, suggesting the emission to be that of an ancient episode of an active galactic nucleus in the central elliptical of the cluster, ESO 470-G020. After reducing archival ATCA data for Abell S1063 we find no evidence of a halo and consider the source to be constructed of blended point sources. We close with a description of a strong double-lobed radio source associated with a nonelliptical host ESO 472-G013, likely a spiral or irregular galaxy, that was found serendipitously whilst searching for diffuse cluster emission. We explore the host within the context of star-formation, and consider the possible origins of the AGN and lobes due to interaction with either the nearby spiral, ESO 472-G012, or a past or ongoing merger event. 


\section{Statement of Originality}

I certify that this work contains no material which has been accepted for the award of any other degree or diploma in any university or other tertiary institution and, to the best of my knowledge and belief, contains no material previously published or written by another person, except where due reference has been made in the text. In addition, I certify that no part of this work will, in the future, be used in submission for any other degree or diploma in any university or other tertiary institution without the prior approval of the Victoria University of Wellington and where applicable, any partner institution responsible for the joint-award of this degree. I give consent to this copy of my thesis, when deposited in the University Library, being made available for loan and photocopying, subject to the provisions of the Copyright Act 1994. I also give permission for the digital version of my thesis to be made available on the web, via the University's digital research repository, the library catalogue and also through web search engines, unless permission has been granted by the University to restrict access for a period of time.

In preparation of this manuscript and this work, I acknowledge Dr. Siamak Dehghan for providing optical density analyses for §ii.2.2.25 and Dr. Qian Zheng who organised, calibrated, and flagged data from the ATCA presented in §iii.2.

List of publications being prepared based on work in this thesis (titles pending):

- Duchesne, S. W., Johnston-Hollitt, M., Offringa, A. R., Pratt, G. W., Zheng, Q., and Dehghan, S., (in prep.) Diffuse galaxy cluster emission at $168 \mathrm{MHz}$ within the Murchison Widefield Array Epoch of Reionization 0-hour field.

- Duchesne, S. W., Johnston-Hollitt, M., and Zheng, Q., (in prep.) The curious AGN associated with the star-forming galaxy ESO 472-G013.

List of publications being prepared, that the author has been involved in during the course of this thesis and topically related (titles pending):

- Johnston-Hollitt, M., Duchesne, S. W., Dehghan, S., and Zheng, Q., (submitted to PASA) Serendipitous discovery of a double-lobed AGN in the diskhosting galaxy PGC 17721.

- Johnston-Hollitt, M., Duchesne, S. W., Zheng, Q., et al., (in prep.) A catalogue of diffuse cluster sources with the GaLactic and Extragalactic All-sky MWA survey.

- Schellenberger, G., Vrtilek, J., David, L., et al., (in prep.) NGC 741 Mergers and AGN feedback on galaxy group scale. 
List of publications that the author has been involved in during the course of this thesis, though not topically related to the work in this thesis:

- Curran, S. J., Duchesne, S. W., Divoli, A., Allison, J. R., (2016). A comparative study of intervening and associated H I 21-cm absorption profiles in redshifted galaxies, Mon. Not. R. Astron. Soc., 462:4197-4207.

- Allison, J. R., Zwaan, M. A., Duchesne, S. W., Curran, S. J., (2016). Using $21 \mathrm{~cm}$ absorption surveys to measure the average H I spin temperature in distant galaxies, Mon. Not. R. Astron. Soc., 462:1341-1350.

- Allison, J. R., Moss, V. A., Macquart, J.-P., Curran, S. J., Duchesne, S. W., et al., (in press.) Illuminating the past 8 billion years of cold gas towards two gravitationally lensed quasars, Mon. Not. R. Astron. Soc. 


\section{Acknowledgements}

First and foremost, I would like to thank my supervisor, Assoc. Prof. Melanie Johnston-Hollitt, who through the course of this work has been a wealth of knowledge and motivation; providing myriad ideas and support for the direction in which this work has progressed. I must also thank her for the funding provided, without which I would not be involved in this research.

I thank Dr. Siamak Dehghan, Dr. Qian Zheng, and Dr. Sara Shakouri, who have been more than helpful and were always willing to answer questions, ranging from "Where do I start with MIRIAD?" to "Do you know how to truncate the bibliography entries for BIBTEX?" I would also like to thank Susannah Keel for pointing out the diffuse emission within Abell S1099.

I would like to thank Dr. Gabriel Pratt, who provided processed X-ray data from deep XMM-Newton observations of a sample of galaxy clusters, and Dr. Philip Edwards who awarded director's discretionary time for observations with the ATCA. I also thank Dr. Emil Lenc who provided the baseline data of the MWA, and Prof. Thomas Reiprich, who provided a look at the X-ray emission from Abell 2496 prior to its public availability. I must also thank Dr. André Offringa who provided the EoR0 field, the image upon which this work is based.

Last, but very much not least, I would like to thank my parents, Tracey and Guy Duchesne, who have supported me throughout this work, but also through all previous studies. 


\section{Contents}

i Introduction $\quad 1$

i.1 Extragalactic radio emission . . . . . . . . . . . . . . . . 1

i.1.1 Synchrotron emission . . . . . . . . . . . . . . . 1

i.1.2 Radio galaxies . . . . . . . . . . . . . . . 5

i.1.3 Clusters of galaxies . . . . . . . . . . . . . 6

i.1.3.1 Relics ................. 7

i.1.3.2 Haloes .................. . . 8

i.1.3.3 Mini-haloes . . . . . . . . . . . . . . . . . . . 10

i.1.3.4 Re-acceleration mechanisms for diffuse emission . . 10

i.2 Radio instruments and surveys at multiple wavelengths . . . . . . . 11

i.2.1 Australian radio telescopes . . . . . . . . . . . . . . . . 11

i.2.1.1 The Murchison Widefield Array . . . . . . . . . . . 12

i.2.1.2 The Australia Telescope Compact Array . . . . . . 13

i.2.2 Survey science . . . . . . . . . . . . . . . . . . . . 13

i.2.2.1 Radio surveys . . . . . . . . . . . . . . . . 13

i.2.2.2 Other surveys and archival data . . . . . . . . 14

ii Diffuse cluster emission within the EoR0 field $\quad 15$

ii.1 The search for diffuse cluster emission . . . . . . . . . . . . . . . 15

ii.1.1 The Epoch of Reionization 0-hour field . . . . . . . . . . . . 15

ii.1.2 Catalogues of galaxy clusters . . . . . . . . . . . . . . 17

ii.1.3 Source detection and measurement . . . . . . . . . . . . 18

ii.1.3.1 Manual source-finding: eyeballing galaxy clusters . 18

ii.1.3.2 Noise and flux densities . . . . . . . . . . . . . . 19

ii.1.3.3 Spectral indices and source sizes . . . . . . . . 21

ii.2 Results from the EoR0 field . . . . . . . . . . . . . . . . . . 23

ii.2.1 Diffuse cluster emission at $168 \mathrm{MHz} \ldots \ldots . . . . . . .23$

ii.2.2 Individual galaxy clusters . . . . . . . . . . . . . 26

ii.2.2.1 Abell $0013 \ldots \ldots \ldots \ldots$. . . . . . . . . . 26

ii.2.2.2 Abell $0022 \ldots \ldots \ldots . \ldots . . \ldots 28$

ii.2.2.3 Abell $0033 \ldots \ldots$. . . . . . . . . . . . . 30

ii.2.2.4 Abell $0085 \ldots \ldots \ldots$. . . . . . . . . . 31

ii.2.2.5 Abell $0122 \ldots \ldots \ldots 33$

ii.2.2.6 Abell $0133 \ldots \ldots \ldots . \ldots \ldots$

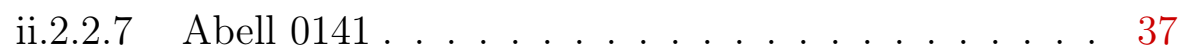

ii.2.2.8 Abell 2496. . . . . . . . . . . . . 39

ii.2.2.9 Abell 2556 and Abell 2554 . . . . . . . . . . . 41

ii.2.2.10 Abell 2680 . . . . . . . . . . . . . . . 42 
ii.2.2.11 Abell 2693 . . . . . . . . . . . . . . . 43

ii.2.2.12 Abell 2721. . . . . . . . . . . . . . 44

ii.2.2.13 Abell 2744. . . . . . . . . . . . . . . 46

ii.2.2.14 Abell 2751 and APMCC 039 . . . . . . . . . 50

ii.2.2.15 Abell 2798. . . . . . . . . . . . . . 51

ii.2.2.16 Abell 2811 . . . . . . . . . . . . . . 52

ii.2.2.17 Abell 3964................. . . 53

ii.2.2.18 Abell 4038 . . . . . . . . . . . . . . . . 54

ii.2.2.19 Abell S0084 . . . . . . . . . . . . . . 55

ii.2.2.20 Abell S1063 . . . . . . . . . . . . . . . . . 55

ii.2.2.21 Abell S1099 . . . . . . . . . . . . . . . . . . . . . . . . . . . . . . 58

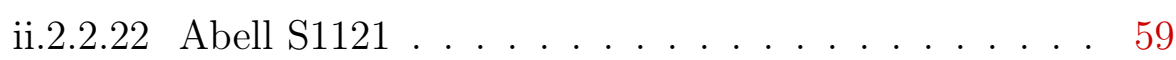

ii.2.2.23 Abell S1136 . . . . . . . . . . . . . 61

ii.2.2.24 PSZ1 G307.55-77.87 . . . . . . . . . . . . 62

ii.2.2.25 RXC J2351.0-1954 . . . . . . . . . . . . 63

ii.2.2.26 MACS J2243.3-0935 . . . . . . . . . . . . . . . . . 66

ii.2.2.27 WHL J235151.0-085929 . . . . . . . . . 68

ii.2.3 On the scaling relations of cluster radio haloes . . . . . . . 69

ii.2.3.1 The $P_{1.4}-L_{\mathrm{X}}$ relation . . . . . . . . . . . . . . 69

ii.2.3.2 The $P_{1.4}-M_{500}$ relation . . . . . . . . . . . . 72

ii.2.4 The detection rate of diffuse cluster emission within the

EoR0 field . . . . . . . . . . . . . . . . . . 73

ii.3 Conclusions about the EoR0 field . . . . . . . . . . . . . 76

$\begin{array}{ll}\text { iii Serendipity and follow-up observations } & 79\end{array}$

iii.1 Diffuse emission within Abell S1136 . . . . . . . . . . . . . . 79

iii.1.1 A dying radio galaxy associated with ESO 470-G020 . . . . 79

iii.1.2 Follow-up analysis with GLEAM . . . . . . . . . . . 81

iii.1.3 Follow-up observations of Abell S1136 at 2.1 GHz . . . . . . 82

iii.1.3.1 Observing and calibrating .......... 83

iii.1.3.2 Imaging the data . . . . . . . . . . . . . . . 84

iii.1.4 Results with the ATCA . . . . . . . . . . 86

iii.1.5 Discussion and conclusion on the nature of the emission within Abell S1136 . . . . . . . . . . . . . . 87

iii.2 Diffuse emission within Abell S1063 . . . . . . . . . . . . . 90

iii.2.1 ATCA data of Abell S1063 . . . . . . . . . . . . . . . . . . 91

iii.2.2 Analysis of the central cluster region . . . . . . . . . . . . . 91

iii.2.3 Conclusion on the nature of the emission within Abell S1063 94

iii.3 A curious AGN host . . . . . . . . . . . . . . . . . . . 95

iii.3.1 Disk galaxies hosting AGN . . . . . . . . . . . . . . 95

iii.3.2 The curious host: ESO 472-G013 . . . . . . . . . . . . 95

iii.3.3 Visible and ultraviolet wavelengths . . . . . . . . . . . . . . 96

iii.3.4 At radio wavelengths . . . . . . . . . . . . . . . . . 97

iii.3.4.1 Radio flux density . . . . . . . . . . . 97 
iii.3.4.2 Spectral energy distribution from $72-8400 \mathrm{MHz}$. . 99

iii.3.4.3 The $P-D$ diagram . . . . . . . . . . . . . 103

iii.3.5 At mid-infrared wavelengths . . . . . . . . . . . . . . . 103

iii.3.6 Star-formation rates . . . . . . . . . . . . . . . . . . . 103

iii.3.7 Scenarios for the origin of the curious morphology . . . . . 106

iii.3.8 Conclusion . . . . . . . . . . . . . . . . . . . 107

$\begin{array}{lr}\text { iv Summary } & 109\end{array}$

A Source-finding and measuring with Witchwood 127

A.1 The floodfill algorithm . . . . . . . . . . . . . . . . . . 129

A.2 Calculating the flux density and other measurements . . . . . . . 129

A.2.1 Flux density . . . . . . . . . . . . . . . . . . . . . 129

A.2.2 Other quantities . . . . . . . . . . . . . . 130 


\section{List of Figures}

i.1 Example spectral energy distributions . . . . . . . . . . . . . . . 4

i.2 Example galaxies with radio emission . . . . . . . . . . . . 5

i.3 Example galaxy cluster . . . . . . . . . . . . . . . 6

i.4 Example radio relics in Abell 4038 and Abell 3667 . . . . . . . . . 8

i.5 Example radio haloes . . . . . . . . . . . . . . . . . . . 9

i.6 MWA baselines . . . . . . . . . . . . . . . . . . . 12

ii.1 The EoR0 field . . . . . . . . . . . . . . . . . 16

ii.2 Flux densities differences between GLEAM and the EoR0 field . . . 20

ii.3 Calibrator flux densities . . . . . . . . . . . . . . . . . . 21

ii.4 Abell $0013 \ldots \ldots \ldots$. . . . . . . . . . . . . . . 27

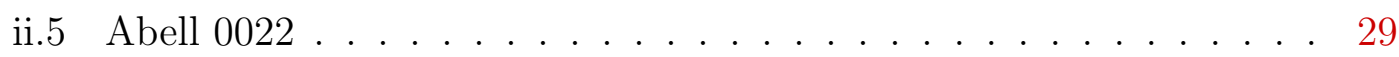

ii.6 Abell $0033 \ldots \ldots \ldots$. . . . . . . . . . . . . . . 30

ii.7 Abell $0085 \ldots \ldots \ldots \ldots$. . . . . . . . . . . . . . . . 32

ii.8 Abell $0122 \ldots \ldots \ldots \ldots$. . . . . . . . . . . . . . . . . 34

ii.9 Abell $0133 \ldots \ldots \ldots$. . . . . . . . . . . . . . . . . . . 35

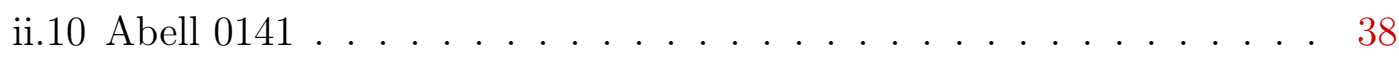

ii.11 Abell $2496 \ldots \ldots$. . . . . . . . . . . . . . . . 40

ii.12 Abell 2556 and Abell $2554 \ldots \ldots$. . . . . . . . . . . 41

ii.13 Abell $2680 \ldots \ldots$. . . . . . . . . . . . . . . . . . . 42

ii.14 Abell 2693 . . . . . . . . . . . . . . . . . . . . . 43

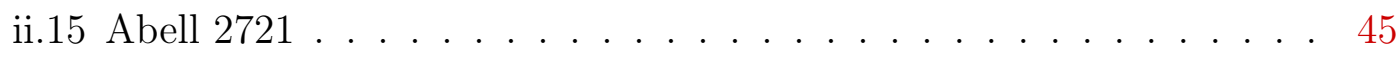

ii.16 Abell 2744 . . . . . . . . . . . . . . . . . . . . . 4 47

ii.17 Abell 2751 and APMCC $039 \ldots \ldots$. . . . . . . . . . . . 49

ii.18 Abell 2798 . . . . . . . . . . . . . . . . . . . 51

ii.19 Abell $2811 \ldots \ldots \ldots$. . . . . . . . . . . . . . . . . 52

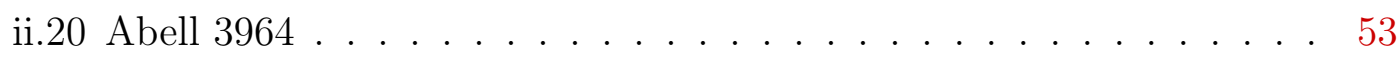

ii.21 Abell $4038 \ldots \ldots \ldots \ldots$. . . . . . . . . . . . . . 54

ii.22 Abell S0084 . . . . . . . . . . . . . . . . . 56

ii.23 Abell S1063 . . . . . . . . . . . . . . . . . . . . . . . . . . . . . . . . . . . . . . 57

ii.24 Abell S1099 . . . . . . . . . . . . . . . . . . . 59

ii.25 Abell S1121 . . . . . . . . . . . . . . . . . . 60 60

ii.26 Abell S1136 . . . . . . . . . . . . . . . . . 62

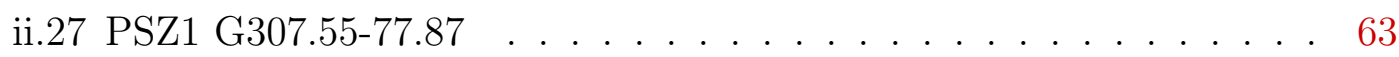

ii.28 RXC J2351.0-1954 f . . . . . . . . . . . . . . . . 64

ii.29 Optical density map of RXC J2351.0-1954 . . . . . . . . . . . . 65

ii.30 MACS J2243.3-0935 . . . . . . . . . . . . . . . 67

ii.31 WHL J235151.0-085929 . . . . . . . . . . . . . . 68

ii.32 The $P_{1.4}-L_{\mathrm{X}}$ diagram . . . . . . . . . . . . . . . . 70 


\section{LIST OF FIGURES}

ii.33 The $P_{1.4}-M_{500}$ diagram . . . . . . . . . . . . . . . . 74

ii.34 Difference in $M_{\mathrm{X}, 500}$ and $M_{\mathrm{YZ}, 500}$ measurements . . . . . . . . . . 76

ii.35 Detections and non-detections for the EoR0 field . . . . . . . . . . . 77

iii.1 RGB image of Abell S1136 . . . . . . . . . . . . . . . . 80

iii.2 SED of the diffuse emission within Abell S1136 . . . . . . . . . 82

iii.3 RFI flagging and calibration strategy for initial sub-bands . . . . . 83

iii.4 ATCA imaging process for Abell S1136 . . . . . . . . . . . . . 85

iii.5 The centre of Abell S1136 . . . . . . . . . . . . . . . . . . . . . . . . . . . . . . 87

iii.6 Stacked ATCA images of Abell S1136 . . . . . . . . . . . . . 88

iii.7 SED of ESO 470-G020 in the $16 \mathrm{~cm}$ band . . . . . . . . . . . . . 89

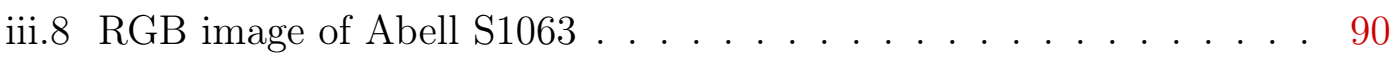

iii.9 ATCA images of Abell S1063 . . . . . . . . . . . . . . . . . 92

iii.10 Three-colour HST image of the centre of Abell S1063 . . . . . . . . 93

iii.11 RGB image of ESO 472-G013 . . . . . . . . . . . . . . . 96

iii.12 GLEAM image of ES0 472-G013 . . . . . . . . . . . . . . . . . . . . . . . . . . 97

iii.13 SED of ESO 472-G013 for the core and lobes . . . . . . . . . . . . . 100

iii.14 Spectral index maps of ESO 472-G013 . . . . . . . . . . . . 101

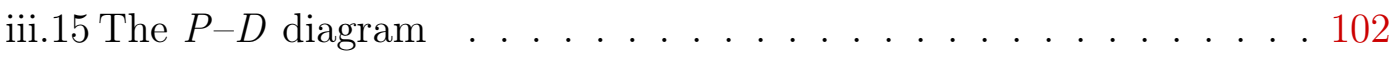

iii.16 WISE colour-colour plot . . . . . . . . . . . . . . . . . . . 104

A.1 Example source-finding with WITCHWOOD . . . . . . . . . 128

A.2 Example output during single-source-finding with WITCHWOOD . . 128 


\section{List of Tables}

ii.1 Existing sky surveys used as auxiliary data to the EoR0 field . . . . 18

ii.2 Summary of diffuse emission within the EoR0 field . . . . . . . . . 24

ii.3 Sources within Abell 2721 . . . . . . . . . . . . . . . . . . . . . 44

ii.4 Sources within RXC J2351.0-1954 . . . . . . . . . . . . . 65

ii.5 Best-fitting parameters from BCES fitting . . . . . . . . . . 73

ii.6 Clusters with diffuse emission and available mass measurements . . 75

iii.1 Measured flux densities of the emission within Abell S1136 . . . . . 81

iii.2 Sub-band properties of the ATCA observations of Abell S1136 . . . 86

iii.3 Properties of ATCA observations of Abell S1063 . . . . . . . . . . . 91

iii.4 Sub-band and image properties of the ATCA observations of Abell S1063 . . . . . . . . . . . . . . . . . 93

iii.5 Flux densities of ESO 472-G013 across the narrow GLEAM bands . 98 iii.6 WISE data for ESO 472-G013 . . . . . . . . . . . . . . . . 103 


\section{§i. Introduction}

\section{§i.1. Extragalactic radio emission}

When observing the sky, radio emission is found to come from within and beyond the Milky Way. Even within our own solar system radio emission is abundant; the positions of Jupiter and the Sun, for instance, need to be taken into account when observing with radio telescopes lest they interfere with observations. Beyond the solar system, but within our galaxy, radio emission may originate from starsvariable (Hewish et al., 1968) or otherwise - or their decaying husks in the form of supernova remnants (SNR, e.g. the Crab Nebula; Bolton et al., 1949), as well as the most compact objects known: the emission from the proposed black hole at the centre of the Milky Way, Sagittarius A* (Balick and Brown, 1974; Brown, 1982; Doeleman et al., 2008).

However it is radio emission of extragalactic origin that is the topic of this work. Extragalactic radio emission was first discovered by Bolton et al. (1949) when they considered the association of the radio source Centaurus A with the optical galaxy NGC 5128. After this, radio sources began to be associated with optical galaxies (e.g. Baade and Minkowski, 1954) and the study of radio galaxies and their associated emission has become an important part of astronomy. It is now known that typical radio galaxies are so-called because of the central monster withinusually an active galactic nucleus (AGN; Scheuer, 1974; Blandford and Rees, 1974) that is centrally located, associated with a black hole and likely an accretion disk. These engines emit across the electromagnetic spectrum, but classes of these object show comparatively strong radio emission. It is not only AGN that will produce radio emission from a galaxy. Star formation - especially in blue, dusty spiral galaxies - will leave a distinctive radio tracer (see e.g. Condon, 1992). These starforming galaxies do not tend to have radio-loud AGN. Instead, their radio emission is generated by the ionized hydrogen (H II) regions which themselves plot potential star-formation sites. There is a final class of extragalactic radio emission that needs to be considered, especially at low radio frequencies: non-thermal, diffuse radio emission associated with galaxy clusters. This emission, associated with the intracluster medium (ICM) rather than any particular galaxy, is found in only a small selection of clusters and the main purpose of this research has been the search for this emission.

\section{§i.1.1. Synchrotron emission}

To understand extragalactic radio emission, we must first consider the radiation mechanism responsible for most extragalactic emission. This is synchrotron emission from charged electrons travelling along helical paths adjacent to magnetic field 
lines. The following is adapted from Rybicki and Lightman (2004). We begin with a discussion of relativistic electromagnetic potentials: the vector potential $\mathbf{A}(\mathbf{r}, t)$ and scalar potential $\phi(\mathbf{r}, t)$. Within the context radiation from charged electrons, we consider the Liénard-Wiechart potentials described by the following

$$
\begin{aligned}
\mathbf{A}(\mathbf{r}, t) & =\frac{\mu_{0}}{4 \pi} \frac{q \mathbf{v}\left(t^{\prime}\right)}{\left(1-\mathbf{R} \cdot \mathbf{v}\left(t^{\prime}\right) / c\right) R}, \\
\phi(\mathbf{r}, t) & =\frac{1}{4 \pi \epsilon_{0}} \frac{q}{\left(1-\mathbf{R} \cdot \mathbf{v}\left(t^{\prime}\right) / c\right) R},
\end{aligned}
$$

where $\mathbf{v}\left(t^{\prime}\right)$ is the particle's measured velocity, $\mathbf{R}=\mathbf{r}(t)-\mathbf{r}_{0}\left(t^{\prime}\right), R=\left|\mathbf{r}(t)-\mathbf{r}_{0}\left(t^{\prime}\right)\right|$, and $t^{\prime}$ is the retarded time, defined as

$$
t^{\prime}=t-\frac{\left|\mathbf{r}(t)-\mathbf{r}_{0}\left(t^{\prime}\right)\right|}{c}
$$

for a particle at position $\mathbf{r}$ measured at $\mathbf{r}_{0}$. This represents the finite speed of light and transmission of information in general. An important result of these potentials arises due to the retarded time - this is the result that charged particles acting within these potentials radiate electric and magnetic fields. From Maxwell's equations, Eq. i.1 and i.2 are used to give the electric and magnetic radiation fields, $\mathbf{E}_{\mathrm{rad}}(\mathbf{r}, t)$ and $\mathbf{B}_{\mathrm{rad}}(\mathbf{r}, t)$ as

$$
\begin{gathered}
\mathbf{E}_{\mathrm{rad}}(\mathbf{r}, t)=\frac{q}{4 \pi \epsilon_{0}}\left\{\frac{(\hat{\mathbf{R}}-\boldsymbol{\beta})\left(1-\beta^{2}\right)}{(1-\hat{\mathbf{R}} \cdot \dot{\boldsymbol{\beta}})^{3} R^{2}}+\frac{\hat{\mathbf{R}}}{(1-\hat{\mathbf{R}} \cdot \dot{\boldsymbol{\beta}})^{3} R} \times \frac{1}{c}[(\hat{\mathbf{R}}-\boldsymbol{\beta}) \times \dot{\boldsymbol{\beta}}]\right\} \\
\mathbf{B}_{\mathrm{rad}}(\mathbf{r}, t)=\frac{1}{c} \hat{\mathbf{R}} \times \mathbf{E}_{\mathrm{rad}}(\mathbf{r}, t),
\end{gathered}
$$

with $\boldsymbol{\beta}=\mathbf{v}\left(t^{\prime}\right) / c$. A further implication of the retarded time is the beaming effect which is more apparent as $v$ approaches the speed of light. Radiation directly in front of the particle's trajectory will be strongest. These results can be used to show that the power radiated from a moving particle is

$$
P^{\prime}=\frac{2 q^{2}}{3 c^{3}}\left|\mathbf{a}^{\prime}\right|^{2}
$$

which, in the instantaneous rest-frame of the particle, with ${a_{0}}^{\prime}=0$ can be written as

$$
P=\frac{2 q^{2}}{3 c^{3}} \gamma^{4}\left(a_{\perp}^{2}+\gamma^{2} a_{\|}^{2}\right)
$$

since $\mathrm{d} W=\gamma \mathrm{d} W^{\prime}$ and $\mathrm{d} t=\gamma \mathrm{d} t^{\prime}$ imply that $P^{\prime}=P$. With this framework, we can now consider synchrotron radiation from a charged particle under the influence of a magnetic field, $\mathbf{B}$ and electric field, E. The four-vector of acceleration for a particle with four-velocity $U^{\nu}$, mass $m_{0}$, charge $e$, and the electromagnetic field tensor $F^{\mu}{ }_{\nu}$, is

$$
a^{\nu}=\frac{q}{m c} F_{\nu}^{\mu} U^{\nu}
$$


This result can be used to show that the equations of motion for a particle acted on by electric and magnetic fields are

$$
\begin{aligned}
\frac{\mathrm{d}}{\mathrm{d} t}(\gamma m \mathbf{v}) & =\frac{q}{c} \mathbf{v} \times \mathbf{B}, \\
\frac{\mathrm{d}}{\mathrm{d} t}\left(\gamma m c^{2}\right) & =q \mathbf{v} \cdot \mathbf{E} .
\end{aligned}
$$

Eq. 1.10 is simply 0 as we are considering the case of no (or negligible) electric field. The implication of this is that $|\mathbf{v}|=$ constant, and from that we can see that $\mathbf{v}_{\|}=$constant and $\left|\mathbf{v}_{\perp}\right|=$ constant which means we have circular motion about the magnetic field in a plane with normal vector in the direction of $\mathbf{B}$. Thus, for a particle moving parallel to the magnetic field the overall motion will be the combination of this circular motion and its inherent linear motion, creating a helical path. We can calculate the frequency of this motion (the gyration frequency, $\left.\omega_{B}\right)$ as

$$
\omega_{B}=\frac{q B}{\gamma m c} .
$$

The perpendicular component of the acceleration is related to this gyration frequency by $a_{\perp}=\omega_{B} v_{\perp}$, and with no parallel acceleration component we can use the relativistic Larmor formula, Eq. i.7, to show that the power radiated by a given particle in the simplest case of parallel motion to the magnetic field is

$$
P=\frac{2 q^{2}}{3 c^{3}} \gamma^{4} \frac{q^{2} B^{2}}{\gamma^{2} m^{2}} \beta_{\perp}^{2} .
$$

This result is more useful if we integrate over all possible pitch angles, $\theta$, which is the angle between the field and the direction of velocity of the electron. Thus $\left\langle\beta_{\perp}{ }^{2}\right\rangle=2 \beta^{2} / 3$ and

$$
P=\left(\frac{2}{3}\right)^{2} \frac{q^{4} \gamma^{2} B^{2}}{c^{2} m^{2}} \beta^{2} .
$$

However this is the power radiated from a single electron. To determine the total power radiated per unit frequency for a population of electrons, $P_{\text {tot }}(\nu)$, we must integrate over the entire population including their corresponding energies. If we assume the electron population can be described by a powerlaw with index $p$, we have $N(E) \mathrm{d} E \propto E^{-p} \mathrm{~d} E$. Thus the total power per unit frequency is

$$
P_{\text {tot }}(\nu) \propto \int_{E_{1}}^{E_{2}} P(\nu) E^{-p} \mathrm{~d} E
$$

where $E_{1}<E<E_{2}$. We define here a critical frequency, $\nu_{c}$, such that

$$
\nu_{c}=\frac{3}{2} \omega_{B} \gamma^{3} \sin \theta
$$

which allows the definition of a dimensionless, constant function $F\left(\nu / \nu_{c}\right)$ proportional to the radiated power of a single electron. Hence Eq. i.14 can be written

$$
P_{\text {tot }}(\nu) \propto \int_{E_{1}}^{E_{2}} F\left(\frac{\nu}{\nu_{c}}\right) E^{-p} \mathrm{~d} E .
$$



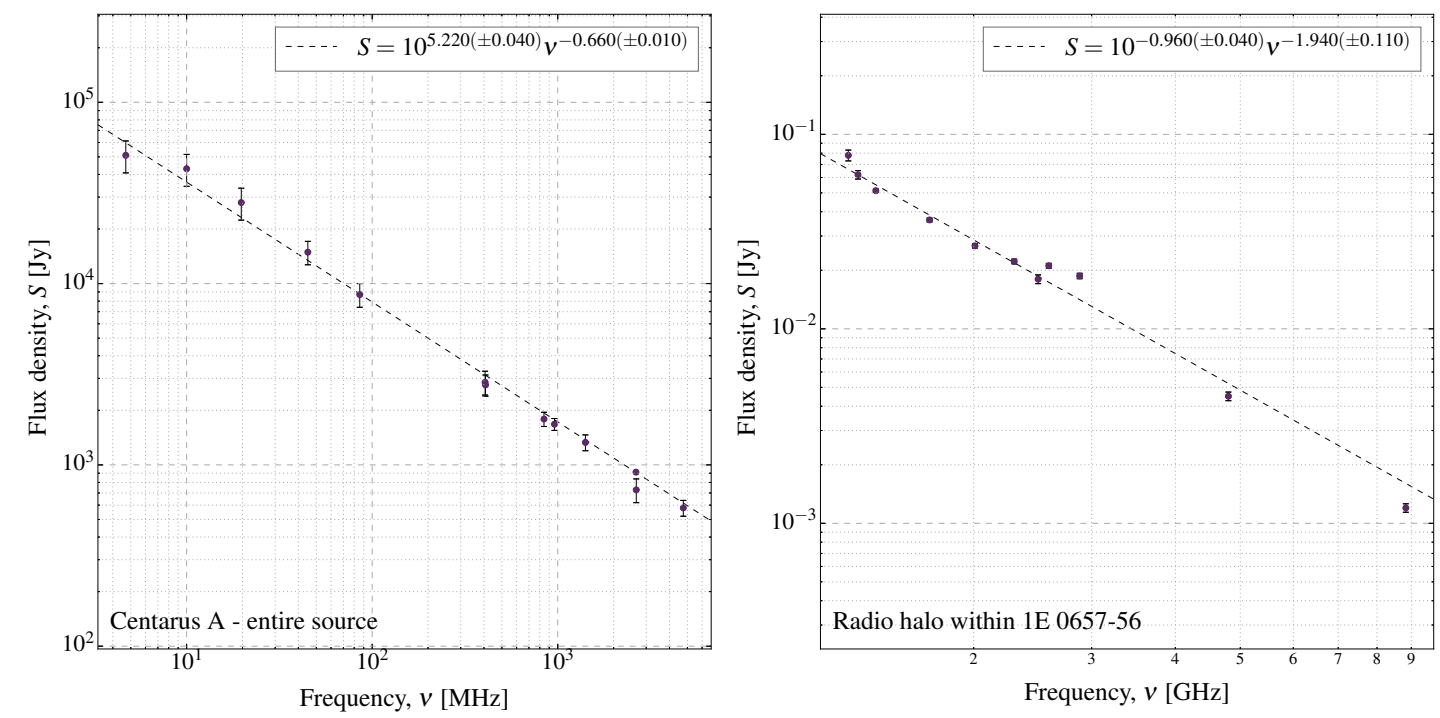

Figure i.1: Example spectral energy distributions. Left: The spectral energy distribution of the radio galaxy, Centaurus A (Alvarez et al., 2000, and references therein). Note that the index is shallower than that calculated by Alvarez et al. as the $4.7 \mathrm{MHz}$ measurement is included here. Right: The same for the radio halo within the Bullet Cluster, 1E 0657-56 (Srinivasan, 2015, and references therein). Note that the index is shallower than that calculated by Srinivasan as all data are included here. Both distributions are shown for illustrative purposes.

With an introduction of a change-of-variable, $x=\nu / \nu_{c}$, and integrating with sufficiently large limits allows the integral to be treated as approximately constant. The resultant expression for $P_{\text {tot }}(\nu)$ is then

$$
P_{\text {tot }}(\nu) \propto \nu^{-(p-1) / 2},
$$

from which we define the spectral index, $\alpha=-(p-1) / 2$. In the literature the spectral index is sometimes defined with the opposite sign and for the this work we use the convention $S_{\nu} \propto \nu^{\alpha}$, where $S_{\nu}$ is the flux density per unit frequency. Fig. i.1 shows two example spectral energy distributions (SEDs) with associated spectral indices.

For a more complete derivation, see the aforementioned Rybicki and Lightman (2004) or e.g. Pacholczyk (1970), Jackson (1975), and Longair (1994). An important observed property of synchrotron radiation is the high-frequency intensity fall-off. This occurs due to higher energy - hence emitting at higher frequencyelectrons losing energy more quickly than those at lower frequencies (see e.g. Scheuer and Williams, 1968). This is known as spectral ageing. It is this phenomenon that gives low-frequency telescopes an advantage in detecting radio emission. For most radio telescopes this comes at the cost of resolution or sensitivity. 

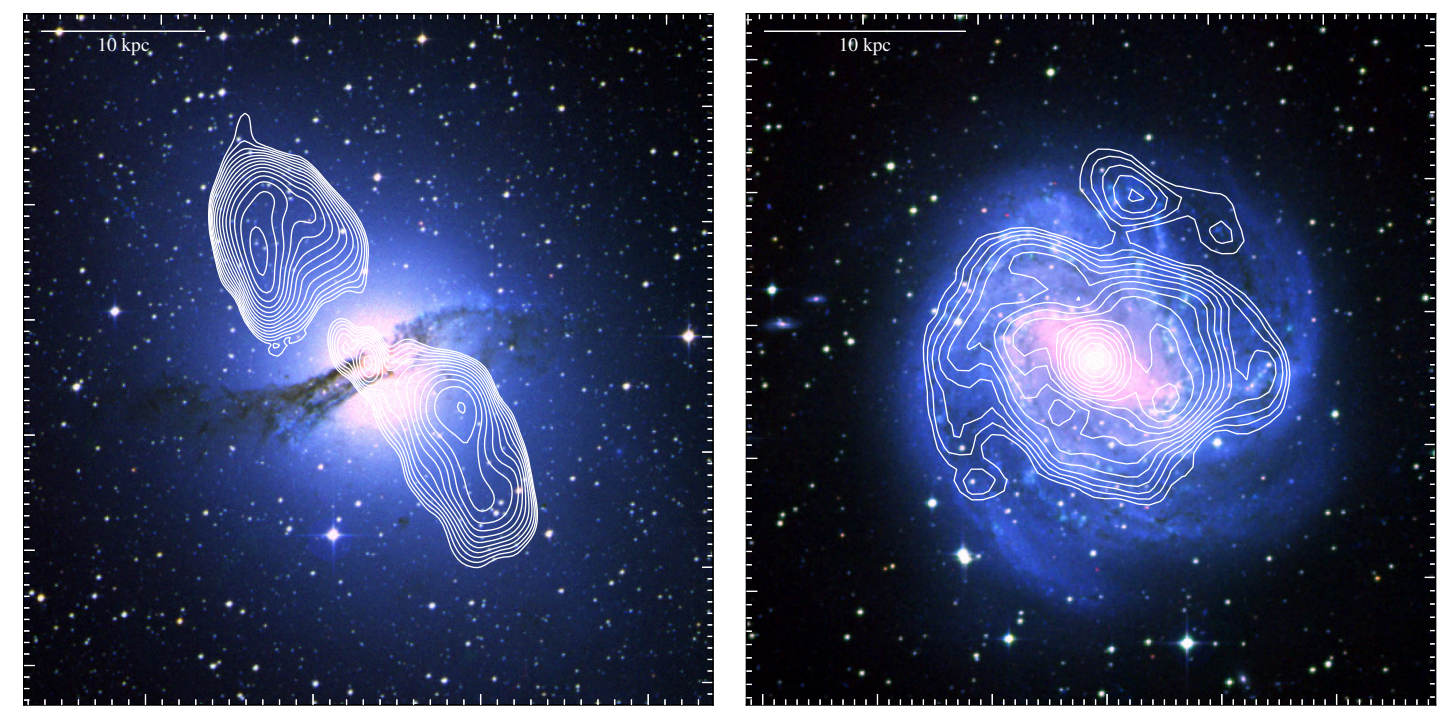

Figure i.2: Examples of radio emission from galaxies. Left: The inner lobes of Centaurus A. The background image is a three-colour image using the Digitized Sky Survey (DSS2) bands, with $150 \mathrm{MHz}$ radio contours from the TFIR GMRT ${ }^{1}$ Sky Survey Alternate Data Release 1 (TGSS ADR1; Intema et al., 2016) overlaid. Right: The star-forming spiral galaxy Messier 83. The background image is as in the left panel, however the radio contours here are at $1.4 \mathrm{GHz}$ from the NRAO VLA ${ }^{2}$ Sky Survey (NVSS; Condon et al., 1998). Figures in this section use survey data that will be expanded upon in §i.2.2.

\section{§i.1.2. Radio galaxies}

When looking at radio images, distant radio galaxies make up the vast majority of sources seen. This is true whether the source is extended or if it is simply a point the size of the synthesized beam of the radio telescope used. A radio galaxy is typically an elliptical galaxy with an AGN. This AGN is hiding a monster withinusually a super-massive black hole $(\mathrm{SMBH})$ that is the primary cause of the radio emission seen. The emission from the SMBH is likely from accreting matter falling into the black hole, with a transfer of gravitational potential energy to radiation of electromagnetic waves. Spiral and lenticular galaxies do not typically show such AGN with emission beyond a few pc, and particularly loud AGN-hosting disk galaxies are seen in only a handful of examples (Centaurus A, the left panel of Fig. i.2 is one such example of a lenticular galaxy hosting a radio-loud AGN). Perpendicular to the rotation axis of the SMBH, a magnetic field is often found, and along these magnetic fields electrons travel at relativistic velocities. This results in synchrotron radiation, as the electron is accelerated in a helical path along the magnetic field lines. In a radio image of such a galaxy seen perpendicular to these magnetic fields, collimated jets of synchrotron emission can be seen as in the left panel of Fig. i.2. These jets can extended up and beyond $1 \mathrm{Mpc}$, though the larger

\footnotetext{
${ }^{2}$ Tata Institute of Fundamental Research Giant Metrewave Radio Telescope

${ }^{2}$ National Radio Astronomy Observatory Very Large Array
} 


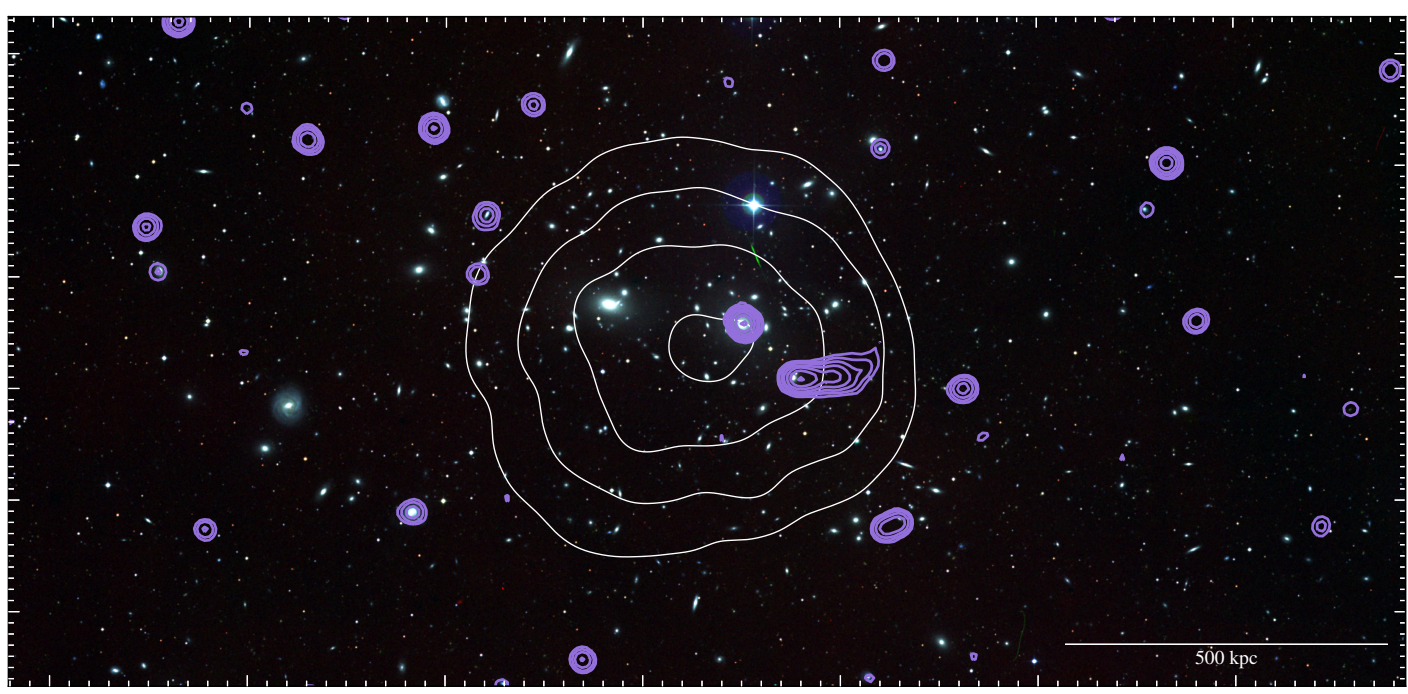

Figure i.3: The Coma Cluster with corresponding three-colour image. The mediumpurple contours are at $1.4 \mathrm{GHz}$ from the NVSS and the white are smoothed archival Chandra data (Obs. ID 13994, PI Sanders). The linear scale is at the redshift of the cluster.

sized jets are typically only seen in under-dense locations and thus will be found either on their own or with a partner or small group, but only rarely seen in large groups or clusters (see e.g. Saripalli et al., 2005). Hotspots of new, fresh electrons (and relatively young or flat spectra) are often seen at the ends of radio lobes, and lobe structures may also feature smaller, inner lobes such as those pictures in the left panel of Fig. i.2 of Centaurus A.

However, radio galaxies with jets are a common occurrence within dense media (e.g. within clusters) and the medium and their motion through it can have a significant effect on their morphology. Miley et al. (1972) describe a class of radio galaxies called head-tail (HT) radio galaxies and suggest that their bent lobes are due to their motion through some medium. There has been work to explore the relationship between the morphology of radio lobes and the environment they find themselves in (see e.g. Gunn and Gott, 1972; Mao et al., 2009; Pratley et al., 2013; Dehghan et al., 2014), and to see whether such objects might act as tracers of dense media such as that within galaxy clusters.

\section{§i.1.3. Clusters of galaxies}

Galaxy clusters are the largest virialized structures in the Universe; thought to be formed hierarchically through mergers of galaxies, groups, and sub-clusters of varying sizes (Peebles, 1980), galaxy clusters are an important tool in understanding how the Universe behaves on the largest scales. The galaxy clusters themselves are primarily dark matter, diffuse gas that makes up the ICM, and the galaxies for which they are named. Clusters are found to host magnetic fields on the order 0.1-1 pG (Clarke et al., 2001; Johnston-Hollitt, 2003; Bonafede et al., 2010). The Milky Way is within the Virgo Super Cluster - a conglomeration of galaxy groups 
and clusters including the Local Group (of which the Milky Way is a member), and the closest proper cluster to us, the Virgo Cluster. Fig. i.3 shows the Coma Cluster, hosting X-ray-emitting plasma and various radio galaxies. Redshift is a measure of wavelength attenuation due to cosmological expansion and peculiar motion of the astronomical object. At cosmological distances, the peculiar motion becomes negligible and redshift becomes an indicator of distance. A discussion of cosmological distance measures can be found in the summary by Hogg (1999).

Galaxy clusters host a wealth of interesting astronomical objects, including the aforementioned HT galaxies. However the purpose of this work is to investigate emission that is not associated with individual galaxies, rather that which is associated with the ICM itself. This type of emission is extended, diffuse, and is thought to be mostly synchrotron in origin. The emission is typically cluster-wide, though ranges in scale from $\sim 100 \mathrm{kpc}$ up to $\sim 2 \mathrm{Mpc}$. The emission in question comes in two main types: radio relics, and radio haloes. They differ in a number of key ways, though have similarities that suggest the same synchrotron emission processes along with similar origins for synchrotron emitting electrons.

\section{§i.1.3.1. Relics}

Radio relics themselves come in two main types: kpc-scale phoenices and Mpcscale cluster relics. For the remainder of this thesis, these will be referred to as phoenices and relics. Fig. i.4 demonstrates two such examples.

Phoenices trace relic synchrotron emission from ancient lobes of long-dead AGN (Komissarov and Gubanov, 1994). After the central engine of a double-lobed galaxy dies, the electrons that once radiated as they spiralled along the magnetic fields running parallel to the black hole rotation axis now radiate along the magnetic field of whatever galaxy cluster they find themselves in. Fundamentally, the synchrotron emission is no different, except that the spectra of phoenices tend to be much steeper than their radio galaxy counterpart $(\alpha<-1.5$; Kempner et al., 2004). This apparent steepening of the spectral index is thought to be due to turbulent re-acceleration and the compression of magnetic fields from the same turbulence in the ICM (Enßlin and Brüggen, 2002). The morphology of phoenices is typically elongated, but still roundish. This apparent shape is unsurprising given their origins as double-lobed radio sources, where the emission might look like a slightly smeared radio galaxy, representing the slow diffusion of the old lobes into the surrounding ICM. Phoenices are often found towards the centre of clusters, and it is likely these objects are the husks of centrally located ellipticals, which often host large-scale radio lobe structures. The typical scale of phoenices ranges from $\sim 100-400 \mathrm{kpc}$, and beyond this size the emission can be considered as proper cluster relic emission. Beyond $400 \mathrm{kpc}$, the turbulent re-acceleration required for the steep SEDs seen is required to be on the cluster-wide scale rather than localised.

Mpc-scale relics are usually more elongated than phoenices, and are found fur-

\footnotetext{
${ }^{3}$ Murchison Widefield Array
} 

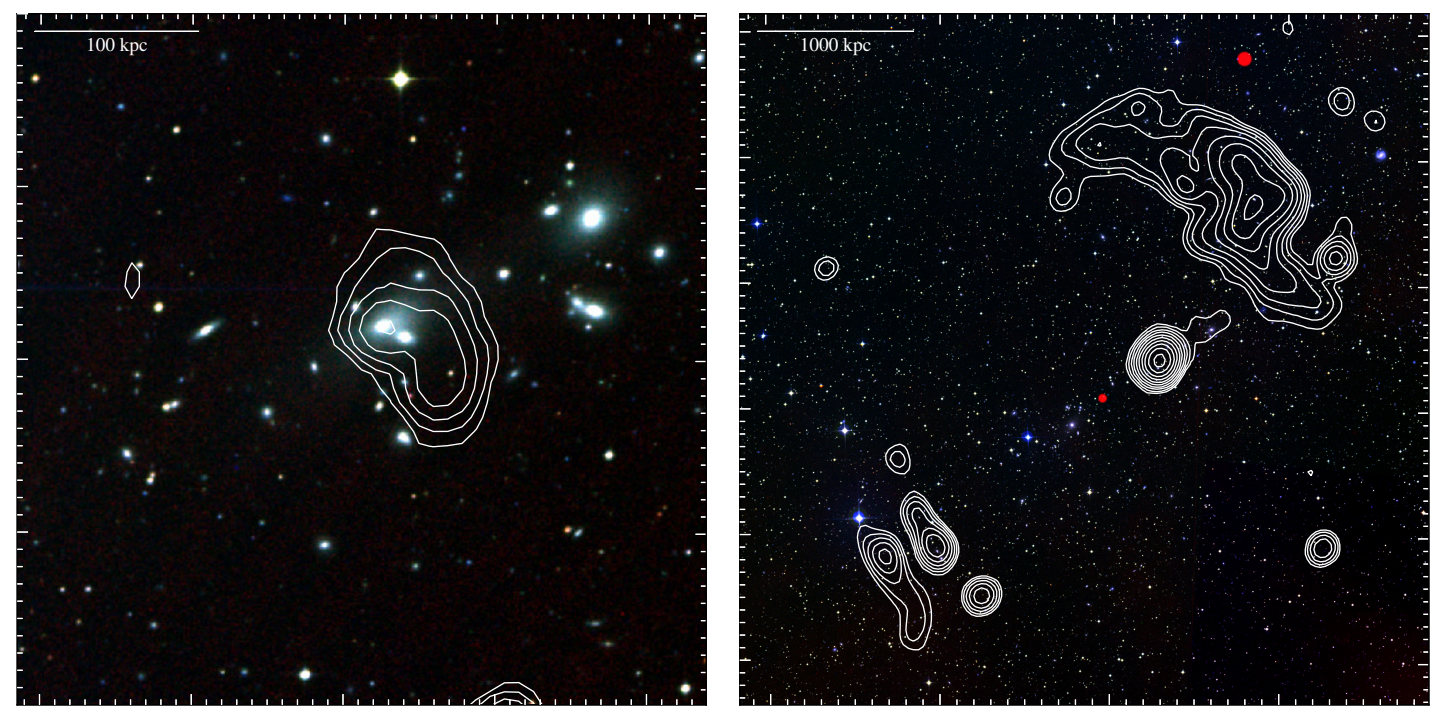

Figure i.4: Example relics. Left: A radio phoenix at the centre of Abell 4038, first reported by Slee and Reynolds (1984). The background is a three-colour image from the DSS2 with $1.4 \mathrm{GHz}$ radio contours from the NVSS. Right: The double relic system associated with Abell 3667, (see e.g. Schilizzi and McAdam, 1975; Röttgering et al., 1997; Johnston-Hollitt, 2003). The background is a three-colour image from the DSS2, with contours at $200 \mathrm{MHz}$ from the GaLactic and Extragalactic All-sky MWA ${ }^{3}$ survey (GLEAM; Wayth et al., 2015) overlaid. The two relics sit on opposite sides of the cluster, a little over $1 \mathrm{Mpc}$ from the cluster's centre. The linear scales in each panel are at the redshifts of the respective clusters.

ther towards the peripheries of clusters. These objects are thought to trace largescale shocks through the ICM during and after massive merger events. These relics are often found to coincide with the major-merger axis, and can come in adjacent pairs known as double relics (e.g. Abell 3667; Johnston-Hollitt 2003, Abell 3376; Bagchi et al. 2006, PSZ1 G108.18-11.53; de Gasperin et al. 2015). These double relics are good observational evidence for shock-re-acceleration as the generation mechanism for the steep SEDs as the double relics are always found on opposing sides of a cluster. Both relics and phoenices exhibit strong fractional polarization which, at the largest scales, can exceed 30 per cent (see e.g Johnston-Hollitt, 2003). Due to projection effects, this can be a good way to distinguish between an intervening relic and a cluster halo, described in the next section.

\section{§i.1.3.2. Haloes}

The large-scale, centrally located, diffuse synchrotron emission found in galaxy clusters is called a halo.

Cluster haloes are centrally located within the cluster, morphologically regular, and are often found to coincide with the X-ray-emitting plasma of the ICM. Two example haloes are shown in Fig. i.5. The X-ray luminosities of clusters with known haloes range from $0.63-35.5 \times 10^{44} \mathrm{erg} \mathrm{s}^{-1}$ in the $0.1-2.4 \mathrm{keV}$ band, 

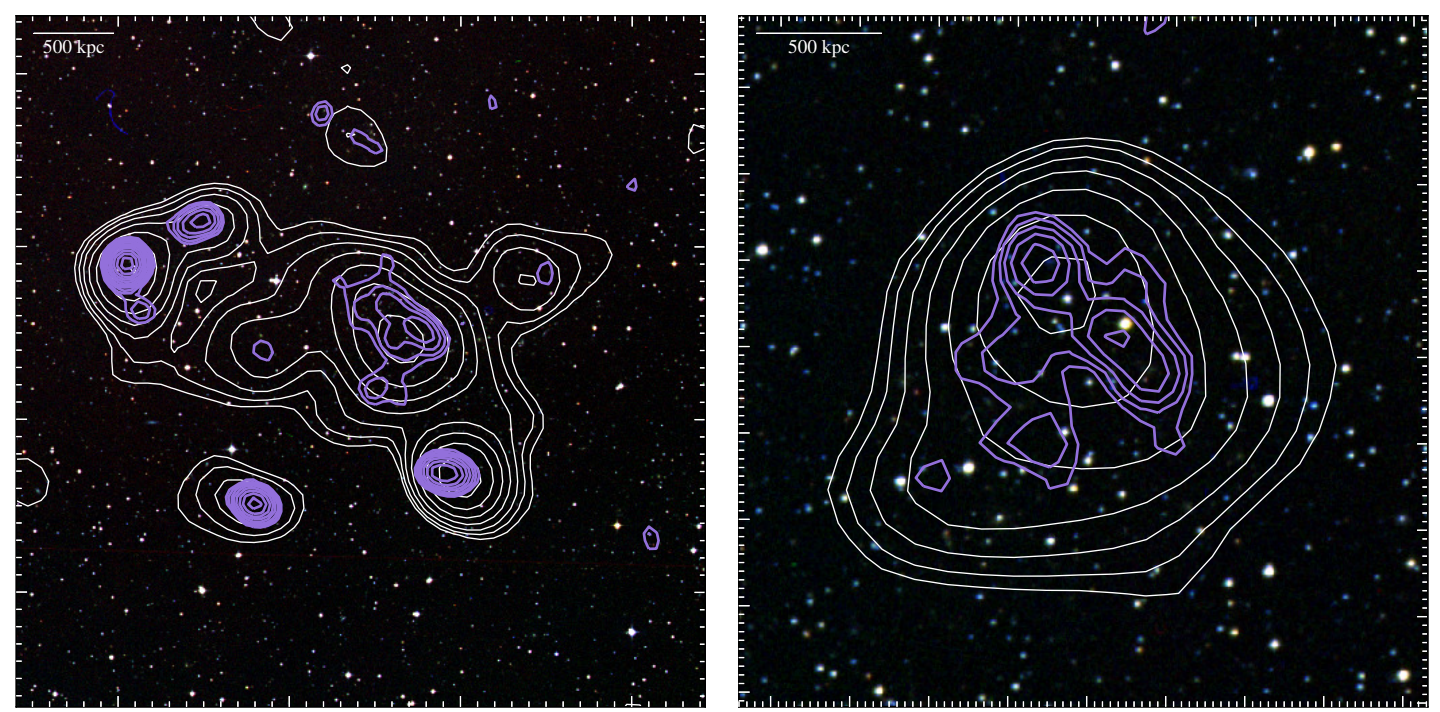

Figure i.5: Left: An example of a giant radio halo in the distant cluster Abell 2163 first reported by Herbig and Birkinshaw (1994). Right: A giant radio halo in RXC J2003.5-2323 detected by Venturi et al. (2007). The background in both panels is a three-colour image with the DSS2, with $200 \mathrm{MHz}$ GLEAM contours in white and $1.4 \mathrm{GHz}$ NVSS contours in medium-purple. The linear scales are at the redshifts of the clusters.

with an average luminosity of $6.7 \times 10^{44} \mathrm{erg} \mathrm{s}^{-1}$. The emission mechanism for the X-ray-emitting plasma is thermal Bremsstrahlung, where electrons experience an acceleration due to their interaction with ions in the plasma. Haloes do not normally show any significant fractional polarisation however this is likely a limitation of the resolution of current-generation radio interferometers (Govoni et al., 2013). The mechanism that generates these radio haloes is still under investigation. The primary, re-acceleration model of halo generation suggests the synchrotron emission occurs after electrons are re-accelerated through mergerdriven turbulence in the magnetised ICM (see e.g. Brunetti et al., 2001; Buote, 2001; Petrosian, 2001; Petrosian and East, 2008; Cassano et al., 2012). An alternate model is that of secondary, hadronic origin (see e.g. Dennison, 1980; Dolag and Enßlin, 2000). In this secondary model, electrons are generated as secondary products of collisions between cosmic ray protons and ICM protons. Pions, a product in these proton-proton collisions, produce electrons as well as $\gamma$-rays. The electrons are subsequently accelerated by magnetic fields and emit synchrotron radiation. This model not only requires $\gamma$-ray emission from clusters, but also that all galaxy clusters host radio haloes albeit at low levels. The synchrotron emission from electrons produced through these proton-proton collisions will be significantly weaker than that seen through re-acceleration via turbulence (Blasi and Colafrancesco, 1999). So far only upper limits for $\gamma$-ray emission have been presented (Prokhorov and Churazov, 2014), and with current generation radio telescopes, the necessary sensitivity to detect haloes generated solely through the secondary model has not been reached. The primary and secondary models 
are not mutually exclusive, and there has been work to combine the two models (e.g. Brunetti and Blasi, 2005; Brunetti and Lazarian, 2011, 2016). The primary model is observationally supported by the fact that only clusters with strong Xray emitting cores are known to have radio haloes. However radio halo detection had been biased toward those clusters hosting highly X-ray luminous plasma as these are the clusters often targeted (e.g. Giovannini et al., 1999; Venturi et al., 2007, 2008; Kale et al., 2013, 2015). Only recently have surveys been conducted to search for diffuse cluster emission without preselecting clusters based solely on their X-ray luminosities. For example, Bernardi et al. (2016) select clusters based on mass, and Shakouri et al. (2016) survey clusters over a wide range of X-ray luminosities.

\section{§i.1.3.3. Mini-haloes}

As well as cluster radio haloes, there also exists a class of diffuse emission called mini-haloes. Mini-haloes show similar properties to cluster haloes, with steep spectral indices, extended structure, and central location within clusters. However they differ observationally in two key ways: mini-haloes are always found coincident with a BCG with significant radio emission, though they are not necessarily associated with this embedded galaxy. The second differences is their observed association with dynamically relaxed clusters, indicating a lack of dynamical requirement suggested for cluster halo formation (for a recent review see Bravi et al., 2016).

\section{§i.1.3.4. Re-acceleration mechanisms for diffuse emission}

Whether haloes or relics, it is likely the mechanism responsible for the observed steep spectra and general properties is that of electron re-acceleration. Due to spectral ageing, for such steep spectra to occur there is a requirement that the electrons responsible for the emission be of great age, at least in comparison to electron populations one might find in normal radio galaxies. As these electrons are part of old populations, they must have energy injected in situ, which can be explained by re-acceleration, either through violent merger events causing largescale shocks through the ICM or through some local ICM turbulence. Jaffe (1977) originally considered a number of possibilities for the observed steep spectrum in the Coma cluster halo. One such possibility includes electrons leaking from one or more cluster radio galaxies, though this is hindered by the long diffusion times and large scales of the cluster halo. However, it is Fermi acceleration that is likely responsible for the properties of both cluster haloes and relics.

Fermi acceleration is of two forms: first order (Fermi-I) and second order (Fermi-II); so-called due to increase in particle energies being proportional to $\beta$ and $\beta^{2}$ respectively (see Protheroe, 1999, and references therein). In the case of Fermi-I, we can consider the scenario of a shock front passed through the magnetised ICM which cleaves the region in two; an upstream component corresponding 
to the pre-shocked ICM and a downstream component corresponding to region that the shock has passed through. Electrons are accelerated by magnetic fields in the upstream region, allowing them to pass through the shock front an into the downstream region. This produces an increase in energy proportional to the velocity of the shock. Within the downstream region, they may be accelerated again by the magnetic fields and may cross back over the shock into the upstream region, increasing the energy further. For $k$ crossings, the energy can be shown to be

$$
E=E_{0}\left(1+\frac{\Delta E}{E}\right)^{k}
$$

for $E_{0}$ the initial energy and $\Delta E$ the energy change due to crossing the shock. Fermi-II acceleration occurs when electrons are accelerated by magnetic clouds of compressed ICM after or during turbulent events. The energy gain is small as energy gain is almost equal to energy loss - electrons colliding with magnetised clouds with velocities in the same directions lose energy whereas those that collide head-on gain energy. The overall energy gain is proportional to $\beta^{2}$, and since $\beta<1$ energy is always less than in Fermi-I acceleration. Fermi acceleration of both orders have been shown via simulations (see Miniati et al., 2001) to be consistent with observations.

Whilst Fermi acceleration (of both types) does well to explain the emission and spectra seen in haloes and relics, radio phoenices may be explained by a different mechanism - adiabatic compression of the magnetic field resulting in the boost to the energy of the ageing electron populations (Enßlin and Gopal-Krishna, 2001). This process revives radio plasma once associated with a long-dead AGN, where the central engine has shut off, and the lobes - now a radio plasma of electrons and magnetic fields - have diffused into the surrounding ICM, eventually having settled to a point where the diffuse pressure balances the pressure exerted by ICM. The radio plasma comprises of magnetic fields and electrons which, in the presence of a shock or turbulence within the ICM, can be compressed adiabatically.

\section{§i.2. Radio instruments and surveys at multiple wave- lengths}

\section{§i.2.1. Australian radio telescopes}

This work makes heavy use of radio data from two prominent Australian telescopes: the Murchison Widefield Array (MWA; Tingay et al., 2013; Bowman et al., 2013) and the Australia Telescope Compact Array (ATCA; Frater et al., 1992). The following section briefly describes these two instruments. 


\section{§i.2.1.1. The Murchison Widefield Array}

The MWA is an array consisting of 128 individual aperture arrays called tiles. The MWA is situated within the Murchison Radio-astronomy Observatory (MRO), which is operated by CSIRO ${ }^{4}$. The MRO lies in an isolated region of Western Australia and has very little radio frequency interference (RFI); other Australian telescopes such as the Australia Square Kilometre Array Pathfinder (ASKAP) also make use of this site for the same reason. In this work, we make use of an deep, $45^{\circ}$ by $45^{\circ}$ image created for use in the study of the Epoch of Reionzation (EoR0). This image will be briefly described in §ii.1.1 and is described in detail in Offringa et al. (2016).

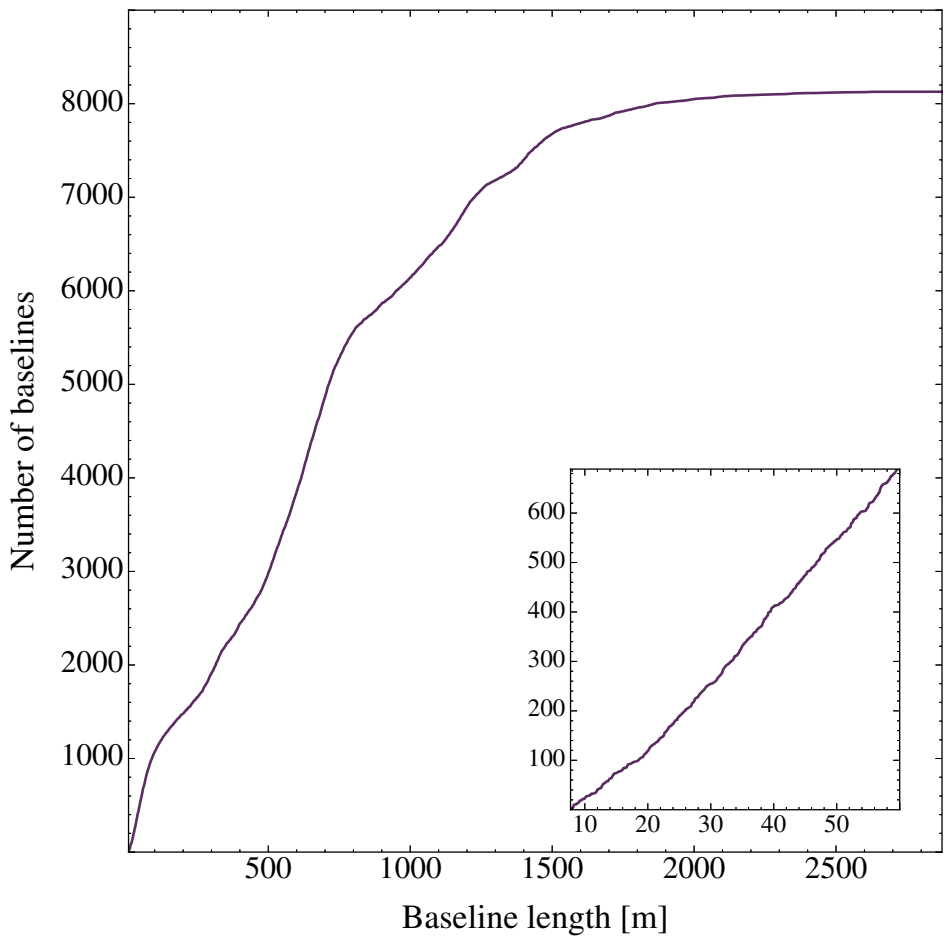

Figure i.6: Number of baselines greater than a given baseline length for the MWA. Inset: As before but for baselines $\leq 60 \mathrm{~m}$ (see Lenc et al., 2016).

The 128 tiles of the MWA perform radio imaging at frequency ranges from 72-300 MHz. The tiles are arranged in groups of 8 , with a receiver connected to each group. The tiles are spaced to attempt to provide smooth $u v$ coverage, which is provided by an $r^{-2}$ radial distribution for the central region, with extra tiles being placed at larger distances. The minimum baseline, or separation of tiles, is $7.7 \mathrm{~m}$, and the maximum is $\sim 3 \mathrm{~km}$. This minimum baseline, as well as number of smaller baselines, provides good sensitivity to extended, diffuse structures. Fig. i.6 shows the number of baselines available greater than a given baseline length with an inset showing the baselines less than $60 \mathrm{~m}$. The largest baseline provides a limit on the angular resolution, as well as the sensitivity to compact structures.

\footnotetext{
${ }^{4}$ Commonwealth Scientific and Industrial Research Organisation
} 


\section{§i.2.1.2. The Australia Telescope Compact Array}

The ATCA is a six-antenna Earth rotation aperture synthesis array operated as part of the Austrlia Telescope National Facility (ATNF) and is located near Narrabri, $~ 550 \mathrm{~km}$ from Sydney, at the Paul Wild Observatory. The six antennas are $22 \mathrm{~m}$ in diameter each, and have a maximum spacing of $6 \mathrm{~km}$ and minimum spacing of $31 \mathrm{~m}$. These spacings allow sensitivity to radio sources that have both extended and reasonably compact structures. The telescope can operate at various frequency bands, between 1.1-105 GHz.

Five of the six antenna stations move along tracks, which allows for variability in the baselines used for observing. The sixth antenna sits on fixed station $3 \mathrm{~km}$ from the fifth, which gives the maximum baseline of the ATCA. Radio images are formed from visibilities, which are correlated signals between pairs of elements of an antenna. To get good sky coverage and sensitivity on the maximum number of spatial scales, the telescope must be allowed to observe for a full 12-hour rotation of the Earth and over a number of the available antenna configurations. Data-reduction and observing strategies for the 1.1-3.1 GHz band are presented in §iii.1.3, and this band is used for a follow-up observation of a peculiar radio source found during the work of §ii.

\section{§i.2.2. Survey science}

Beyond the two radio telescopes described in §i.2.1, survey data has been utilised in this work. In this section, we briefly detail the various sky-surveys, at various wavelengths, that have been used as part of this research.

\section{§i.2.2.1. Radio surveys}

At radio wavelengths, there are four main sky surveys that are used in this work. Firstly, the NVSS (Condon et al., 1998) at 1.4 GHz which covers the entire northern sky down to declinations of -40 degrees. The NVSS has a resolution of 45 arcsec by 45 arcsec and has an average rms noise of approximately $0.45 \mathrm{mJy} \mathrm{beam}^{-1}$. Recently the TGSS (ADR1; Intema et al., 2016) at $147.5 \mathrm{MHz}$ was released as a low-frequency counterpart to the NVSS. The TGSS covers the northern sky and continues its coverage down to declinations of approximately -53 degrees. The TGSS has an average rms of approximately $3.5 \mathrm{mJy}^{\text {beam }}{ }^{-1}$ though is not so sensitive to some diffuse emission as the higher resolution of approximately 25 arcsec by 25 arcsec resolves out flux from extended emission. The southern counterpart to the NVSS is the Sydney University Molonglo Sky Survey (SUMSS; Bock et al., 1999; Mauch et al., 2003) at $843 \mathrm{MHz}$ and covers the southern sky up to a declination of -30 degrees. The resolution is equivalent to the NVSS, and its rms is approximately 2-4 mJy beam ${ }^{-1}$ depending on the particular field and if there are any particularly bright and confusing sources nearby. Finally, there is the VLA Low-frequency Sky Survey redux (VLSSr; Lane et al., 2014) at $74 \mathrm{MHz}$ which is 
another northern survey, extending down to -30 degrees. When comparing to the 6C (Hales et al., 1991) and 8C (Rees, 1990) surveys Lane et al. find that the VLSSr has up to a 30 per cent discrepancy in its flux scale for sources below $1 \mathrm{Jy}$, and so is only used in this work when that is not the case. The average rms for the VLSSr is approximately 100 mJy beam $^{-1}$ and its resolution is $75 \operatorname{arcsec}$ by 75 arcsec.

Those four surveys present the majority of the auxiliary data used in this work. During the course of this research, the GLEAM survey (Wayth et al., 2015) was released which covers the frequency range $72-231 \mathrm{MHz}$. This range is broken up into twenty $7.5 \mathrm{MHz}$ sub-bands, as well as a single $60 \mathrm{MHz}$ band centred on 200 $\mathrm{MHz}$ and three $30 \mathrm{MHz}$ bands centred on 88, 118, and $154 \mathrm{MHz}$. The coverage of GLEAM is from approximately +25 degrees and includes the entire southern sky. The rms varies between the sub-bands, but ranges from approximately 12 $70 \mathrm{mJy}_{\text {beam }}{ }^{-1}$ for the highest and lowest bands respectively. GLEAM is a useful counterpart to the aforementioned surveys, though is not used for research detailed in §ii as there is a larger study of diffuse cluster emission detected with GLEAM being prepared by Johnston-Hollitt et al. The GLEAM survey is used in §iii for follow-up work.

\section{§i.2.2.2. Other surveys and archival data}

The research makes heavy use, however qualitatively, of the Digitized Sky Survey (DSS2) images. The DSS2 is an all-sky survey in the red, blue, and near-infrared bands of the Oschin Schmidt Telescope on Palomar Mountain and the UK Schmidt Telescope at the Anglo-Australian Observatory. Each telescope covers the northern and southern portions of the survey respectively. For the purpose of this work, the DSS2 is used to optically identify potential host galaxies and to visually confirm the centres of clusters. The RÖentgen SATellite (ROSAT; Trümper, 1984) All-Sky Survey (RASS; Voges et al., 1999) is also used as a quick method of checking for significant soft-band X-ray emission near clusters, though no results from RASS are presented in this work. Archival Chandra data with the Advanced CCD Imaging Spectrometer (ACIS) instrument and XMM-Newton data with the European Photon Imaging Camera (EPIC) instrument are used where available for similar reasons, and in a select few clusters we utilise deep (>30 ks exposure) X-ray images from the Representative XMM-Newton Cluster Structure Survey (REXCESS; Böhringer et al., 2007; Pratt et al., 2009) which have been reduced by Dr. Gabriel Pratt. Additionally, Dr. Gabriel Pratt has reduced archival XMM-Newton which is used in §ii. Finally, we make use of AllWISE results of the Widefield Infrared Survey Explorer (WISE; Wright et al., 2010; Mainzer et al., 2011) which covers the sky in mid-infrared with bands at 3.4, 4.6, 12, and $22 \mu \mathrm{m}$. These data are used in §iii within the context of star-formation from a curious galaxy. 


\section{§ii. Diffuse cluster emission within the EoR0 field}

The primary goal of this work has been the search for diffuse cluster emissionrelics and haloes. These objects are of interest as they trace cluster merger events. These events are important in understanding the way in which the Universe evolves through large-scale structure formation. Due to the steep spectral indices of such diffuse emission, low-frequency radio observations are more likely to be successful in detection, and in particular the MWA provides a useful tool to commence such a search. We are able to detect new haloes, relics, phoenices, and further re-observe previously detected emission and provide new measurements of these sources. Finally, we are able to explore cluster haloes within the context of scaling relations between halo properties and cluster properties.

\section{§ii.1. The search for diffuse cluster emission}

\section{§ii.1.1. The Epoch of Reionization 0-hour field}

The cosmological Epoch of Reionization (EoR) is thought to be traced by the redshifted neutral hydrogen $(\mathrm{HI})$ 21-cm line occurring somewhere in the vicinity of 50-200 MHz. A method used to attempt to detect this signal is using an interferometric telescope, such as the GMRT EoR experiment (Paciga et al., 2013), the LOFAR ${ }^{1}$ EoR project (Yatawatta et al., 2013), the MWA EoR project (Bowman et al., 2013; Offringa et al., 2016), and PAPER ${ }^{2}$ (Ali et al., 2015).

As part of the MWA EoR project Offringa et al. present a $45^{\circ} \times 45^{\circ}$ image centred on $\left(\alpha_{\mathrm{J} 2000}, \delta_{\mathrm{J} 2000}\right)=\left(00.00^{\circ},-27.00^{\circ}\right)$, at a frequency of $168 \mathrm{MHz}$ called the EoR0 field. This field is shown in Fig. ii.1. The image is obtained from 45 hours of integration and has a resolution of $\sim 2.3$ arcmin. The EoR0 field is the deepest, confusion limited image made with the MWA. As mentioned in §i.2.1.1, the large number of short $(\leq 60 \mathrm{~m})$ baselines makes the MWA a powerful tool to investigate extended, diffuse emission, as the shorter baselines of an interferometric telescope provide sensitivity to large-scale structure. Data collection, reduction, and imaging for the field used here is explained in detail in Offringa et al. Whilst the primary purpose of the EoR0 field is the study of EoR, the image itself is incredibly sensitive, reaching near the centre of the image down to $\sim 2.3$ mJy beam $^{-1}$ and increasing out towards the edges and in particular corners up to $\sim 100$ mJy beam $^{-1}$. This surface brightness sensitivity makes the EoR0 field useful in the search for steep spectrum cluster haloes and relics. The R.A. and decl. range used here is

\footnotetext{
${ }^{1}$ Low-Frequency Array

${ }^{2}$ Precision Array for Probing the Epoch of Reionization
} 


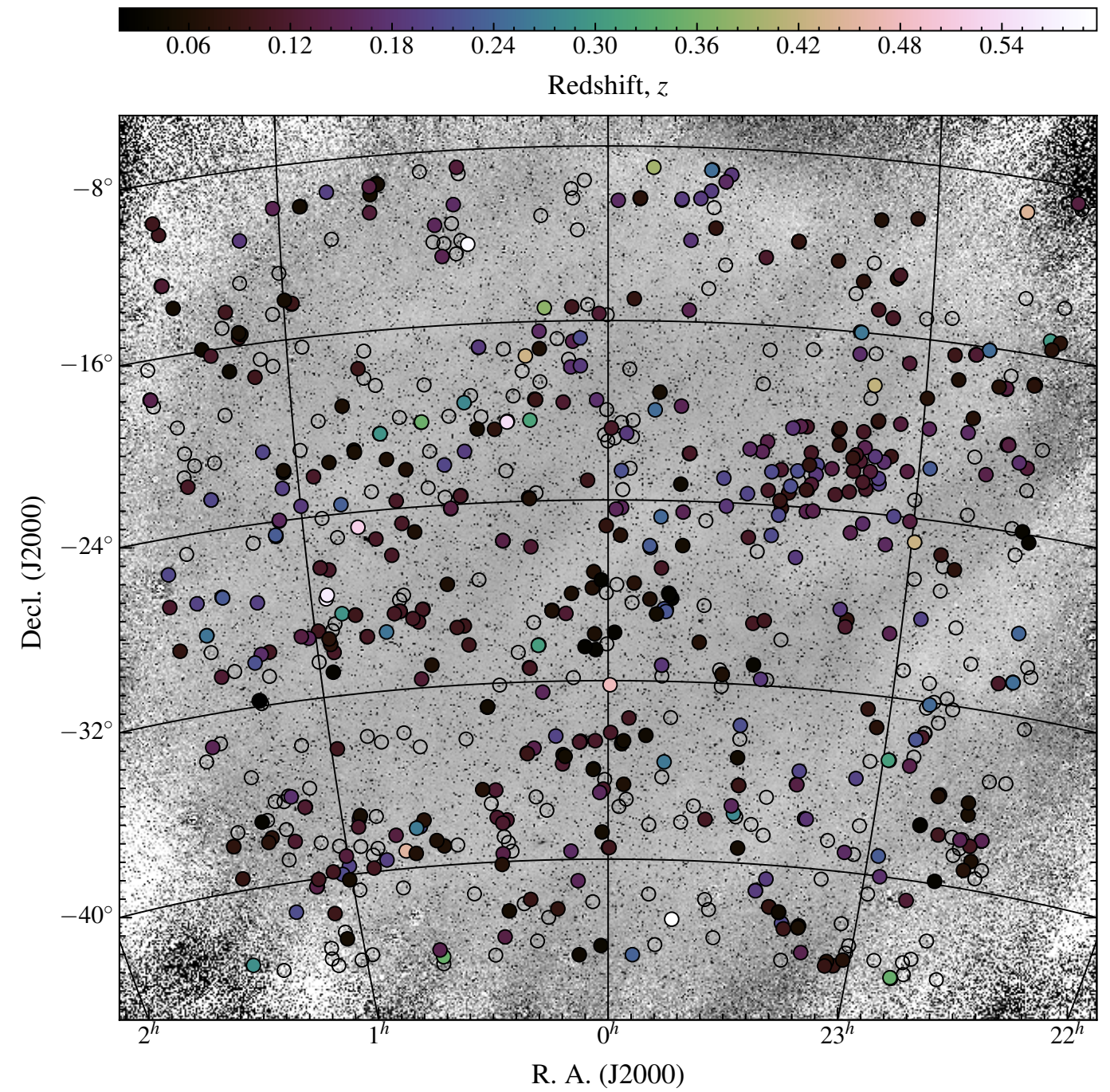

Figure ii.1: The central $\sim 42^{\circ}$ of the Epoch of Reoinization 0-hour field. Overlaid are the positions of galaxy clusters from the Abell catalogues (Abell et al., 1989), Meta-Catalogue of X-ray detected Clusters of galaxies (MCXC; Piffaretti et al., 2011), and the Planck catalogue of Sunyaev-Zel'dovich sources (PSZ1; Planck Collaboration et al., 2015a). We cut the sample of clusters to attempt to avoid the edges of the image where the noise is highest. The filled circles are coloured according to their redshift, and unfilled circles are those without a measured redshift. The sample of clusters is described in §ii.1.2. Note the side-lobe structure of the primary beam appearing in the corners of the image. The colourmap of the redshift distribution is an implementation of CUBEHELIX (Green, 2011). This colourmap is used in various places throughout this work. 
as follows: $\left(337.48^{\circ} \leq \alpha_{\mathrm{J} 2000} \leq 22.49^{\circ}\right)$ and $\left(-44.69^{\circ} \leq \delta_{\mathrm{J} 2000} \leq-08.61^{\circ}\right)$, which is chosen to cut out the most significant noise in the corners and edges of the image.

\section{§ii.1.2. Catalogues of galaxy clusters}

Within the EoR0 field we searched for diffuse emission within a $\sim 2$ Mpc radius around clusters within the following catalogues: Abell revised North, South, and Supplementary (Abell et al., 1989, hereafter ACO) catalogues with 5250 clusters; the Meta-Catalogue of X-ray detected Clusters of galaxies (Piffaretti et al., 2011, hereafter MCXC), with 1743 clusters; the Planck catalogue of Sunyaev-Zel'dovich sources (Planck Collaboration et al., 2015a, hereafter PSZ1) with 1227 clusters. The three catalogues differ in how clusters were detected. For the ACO catalogue, clusters were detected by eye from optical plates. For the MCXC, Piffaretti et al. (2011) compiled and homogenised a collection of X-ray detected clusters from a number of individual catalogues. One of the main purposes of this collection was to remove duplicate entries for the same clusters, but also to actually distinguish between cluster X-ray sources and other extragalactic X-ray sources. The resulting catalogue is therefore not only accurate in its definition of a cluster, but also has accompanying cluster properties, such as redshift and X-ray luminosity. PSZ1 is a catalogue of clusters created from Sunyaev-Zel'dovich (SZ; Sunyaev and Zeldovich, 1970) measurements, where SZ is the effect of the ICM on $\mathrm{CMB}$ photons which provide a tell-tale sign of large mass concentrations, hence clusters. Between the three catalogues there is significant overlap, but within the region encompassed by the EoR0 field, and excluding those clusters that lie too far to the edge of the image, this constitutes 668 unique clusters, 505 unique to ACO, 70 unique to MCXC, and 19 unique to PSZ1, with 24 clusters present in all three catalogues.

All clusters are checked systematically for diffuse cluster emission except 217 clusters in the ACO catalogue without a redshift. For clusters without a redshift we are unable to determine the projected linear distance from the cluster centre, which makes determining if emission is part of the cluster difficult if not at the centre. Whilst this does not pose much problem for haloes, we also consider that ACO clusters without a redshift are unlikely to have auxiliary data in the form of cluster mass, X-ray luminosities, or information on cluster members. We do note, however, that a subset of the PSZ1 cluster do not have a measured redshift though they are searched regardless as they hve auxiliary measured properties. Further, cluster emission serendipitously found in clusters not part of the aforementioned catalogues is investigated when noticed. 
Table ii.1: Existing sky surveys used as auxiliary data to the EoR0 field.

\begin{tabular}{lcccc}
\hline \hline Survey & $\begin{array}{c}\text { Frequency } \\
\text { MHz }\end{array}$ & $\begin{array}{c}\text { Declination } \\
\left(\mathrm{J}_{2000}{ }^{\circ}\right)\end{array}$ & $\begin{array}{c}\text { Resolution } \\
\left({ }^{\prime} \times{ }^{\prime \prime}\right)\end{array}$ & $\begin{array}{c}\sigma_{\text {rms }} \\
\left(\mathrm{mJy} \mathrm{beam}^{-1}\right)\end{array}$ \\
\hline EoR0 & 168 & $-44.69 \leq \delta \leq-08.61$ & $138 \times 138$ & $\gtrsim 2.3$ \\
NVSS & 1400 & $\geq-40$ & $45 \times 45$ & $\sim 0.45$ \\
SUMSS & 843 & $\leq-30$ & $45 \times 45 / \sin (\delta)$ & $\gtrsim 2$ \\
TGSS & 147.5 & $\gtrsim-53$ & $25 \times 25 / \cos (\delta-19)$ & $\sim 3.5$ \\
VLSSr & 74 & $\geq-30$ & $75 \times 75$ & $\gtrsim 100$ \\
\hline
\end{tabular}

\section{§ii.1.3. Source detection and measurement}

\section{§ii.1.3.1. Manual source-finding: eyeballing galaxy clusters}

While source-finding algorithms exist and are put to good use to produce pointsource catalogues, automated source-finding can miss the extended, low surface brightness haloes and relics within clusters. Therefore the EoR0 field is searched by eye for diffuse emission. We use auxiliary data in the form of sky surveys, which are described in §i.2.2, however we do not use the GLEAM survey here as the results of a GLEAM diffuse emission search will be published elsewhere. Table ii. 1 summarises the surveys used, comparing properties to the EoR0 field.

To determine the nature of detected emission, we look for the following:

(i.) high-frequency counterparts $(1.4 \mathrm{GHz}$ and $843 \mathrm{MHz})$,

(ii.) low-frequency counterparts (147.5 and $74 \mathrm{MHz}$ ),

(iii.) optical identifications, and

(iv.) X-ray emission coincident with centrally located radio emission.

(i) and (ii) are used as an easy method of checking if we are looking at blended point sources. (i) gives a quick insight into the spectral index of the source. With significant high frequency emission, at least comparably to $168 \mathrm{MHz}$, a flat spectral index is present which is very uncharacteristic of diffuse cluster emission. (iii) is important as cluster haloes and relics are not associated with an optically visible galaxy, though in the case of cluster haloes there is expected to be a concentration of optically visible galaxies as the halo should be centrally located. If an optically visible galaxy is found at the peak of the diffuse emission or between two lobes, then the likelihood is that of extended, disturbed, or otherwise normal lobes of a radio galaxy. (iv) allows us to confidently classify centrally located diffuse emission as a cluster halo or relic. In particular, Chandra or XMM-Newton observations are detailed enough to provide the position and any elongation of the X-ray emission relative to any centrally located diffuse radio emission. With these points forming the foundations of our search, we eyeballed the subset of clusters described in §ii.1.2 and noted emission that would fall into the category of either halo or relic. Once these objects are found, we wish to look at their spectral properties, including their integrated flux densities over the entire surface of the emission and the associated 
spectral energy distributions (SED) and spectral indices.

\section{§ii.1.3.2. Noise and flux densities}

The EoR0 field is a large image that has greatly varying rms noise throughout. The corners of the image feature heavy noise due to the primary beam null. Offringa et al. (2016) use BANE ${ }^{3}$, a tool that is packaged with the source-finding software, AEGEAN (Hancock et al., 2012), to estimate noise throughout the EoR0 field. The mean noise level is calculated to be $3.2 \pm 0.6 \mathrm{mJy}_{\text {beam }}{ }^{-1}$ for the central $10^{\circ}$ of the image. Large-scale diffuse structure of Galactic origin is seen streaking the image which leads to higher rms noise, however in regions with no Galactic emission the rms can be seen to be as low as $\sim 2$ mJy beam $^{-1}$.

It is also vital to carefully estimate local noise in any ancillary data used at the position of any emission as we are looking at objects that often have emission at or barely above the $3 \sigma_{\text {rms }}$ level at $168 \mathrm{MHz}$. Table ii.1 gives the lower estimate of the rms for each of the surveys, however this varies significantly over the EoR0 field. For the NVSS and TGSS surveys, to obtain local rms values we simply consult the catalogues (Condon et al., 1998; Intema et al., 2016, respectively) and use the local rms noise of the nearest catalogue source. In the case of SUMSS, since no local rms is given in its catalogue (Murphy et al., 2007), we inspect each image and give an estimate of the rms which is usually on the order of $2-4 \mathrm{mJy} \mathrm{beam}^{-1}$, depending quite strongly on whether there is a bright source in the field creating significant imaging artefacts. For the VLSSr images, we use 100-500 mJy beam ${ }^{-1}$, again depending on the individual images.

The EoR0 field does not have a well-defined synthesized beam, which means that integrated flux densities across the field will be inconsistent with other radio data, and we find that the integrated flux density measurements of the EoR0 field differed by a systematic factor of approximately 30 per cent when compared to the nearly equivalent $162-170 \mathrm{MHz}$ band in the recently released GLEAM survey. Fig. ii.2 shows this discrepancy. Sources are measured with AEGEAN on both the EoR0 field and the $162-170 \mathrm{MHz}$ band, within a 5 degree region at the centre of the EoR0 field. Only sources that fit the following criteria are compared:

(i.) $0.1 \leq S_{\nu} \leq 10 \mathrm{Jy}$,

(ii.) $(a b) /\left(B_{\text {maj }} B_{\text {min }}\right)<1.5$,

(iii.) sources in each image are within 5 arcsec of each other,

where $a$ and $b$ are the semi-major and semi-minor axes of the fitted ellipses, and $B_{\text {maj }}, B_{\text {min }}$ are the FWHM of the synthesized beam. Uncertainties are only applied to the flux densities in the $162-170 \mathrm{MHz}$ GLEAM band, and are the quadrature sum of all uncertainties applicable - i.e. the uncertainty in the fitting by AEGEAN and the flux density scale uncertainties inherent to GLEAM and the EoR0 field. The fitting is a weighted least-squares fit, with resulting equation, however we do not use this scale for calibration.

\footnotetext{
${ }^{3}$ https://github.com/PaulHancock/Aegean/wiki/BANE
} 


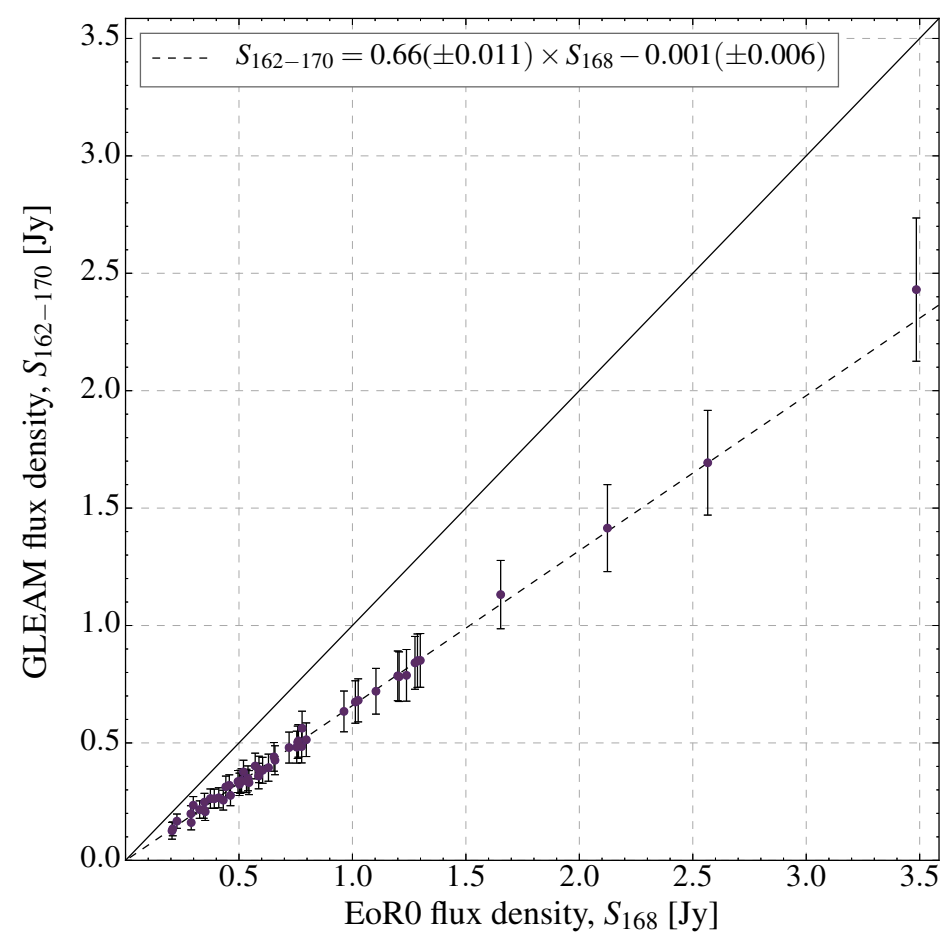

Figure ii.2: Flux density differences for sources between 0.1 and $10 \mathrm{Jy}$ in a 5 degree region of the EoR0 field, comparing with the $162-170 \mathrm{MHz}$ band of GLEAM. The dashed line is a weighted least-squares fit to the data, and the solid line is the expected 1:1 fit. Note that uncertainties in the y direction are the quadrature sum of all applicable uncertainties in the data. Note that the selection criteria included sources up to $10 \mathrm{Jy}$ but no sources were found above 3.5 Jy in this central region.

To scale the integrated flux densities in the EoR0 field we choose six reasonably bright (2-6 Jy) unresolved sources, exhibiting no side-lobe structure and no blending with nearby sources. All six sources show no deviation from a simple power law between $72-231 \mathrm{MHz}$. Fig. ii.3 shows the six calibrators with predicted $168 \mathrm{MHz}$ flux density against measured flux density. From the resultant fit we find a factor $0.690 \pm 0.051$ to be used for calibration of measured integrated flux densities, and this calibration is used through the remainder of this paper.

Flux densities of sources are either calculated by purpose-built PYTHON code ${ }^{4}$ or simply using AEGEAN if sources are blended, as the aforementioned PYTHON code does not fit sources, and assumes each source is discrete. We do not use AEGEAN for all sources as AEGEAN is intended as a point-source-finder, and will give the best results measuring such sources. Each flux measurement has an uncertainty, $\sigma_{S_{\nu}}$, calculated as

$$
\sigma_{S_{\nu}}=\sqrt{\left(0.05 \times S_{\nu}\right)^{2}+\left(0.051 \times S_{\nu}\right)^{2}+\left(\sigma_{\mathrm{rms}} \sqrt{N_{\text {beam }}}\right)^{2}} \quad[\mathrm{Jy}]
$$

where $N_{\text {beam }}$ is the number of beams crossing the extended source. The first term in Eq. ii. 1 is an additional uncertainty added due to flux density scaling in the

\footnotetext{
${ }^{4}$ See Appendix A for details.
} 


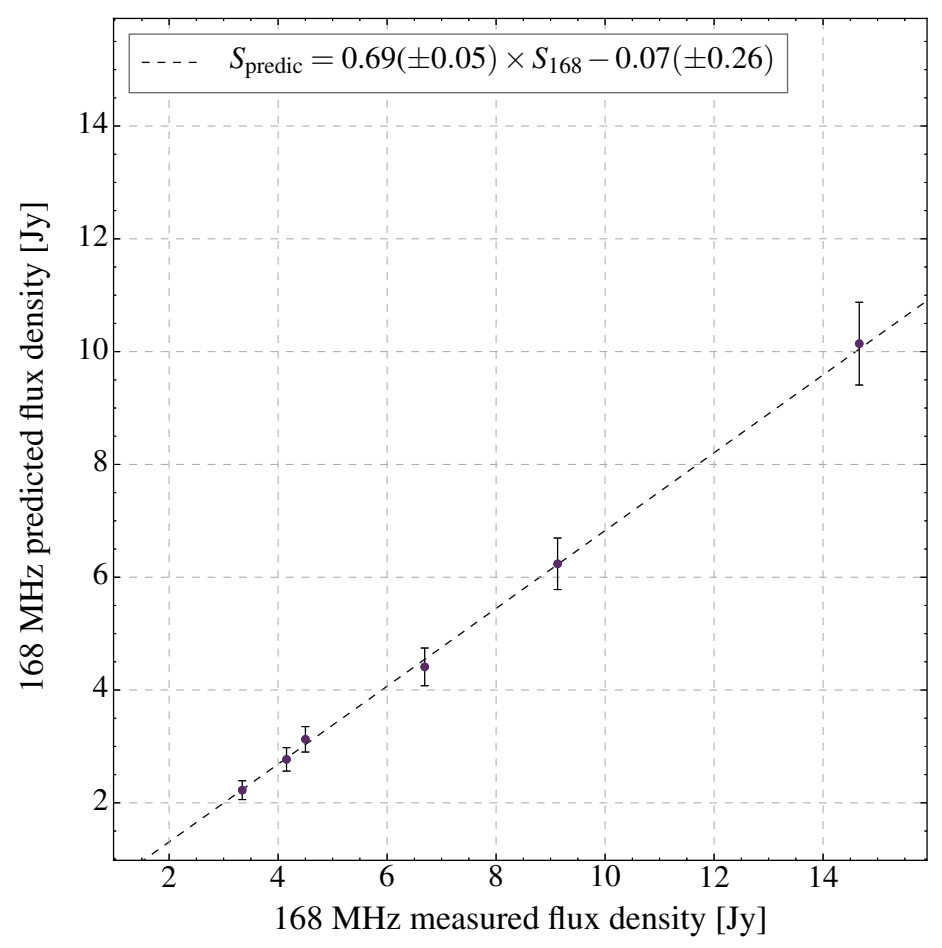

Figure ii.3: Flux densities as measured within the original EoR0 field for the six calibrators, along with their predicted $168 \mathrm{MHz}$ flux density after calculating a spectral index across the GLEAM bands. The dashed line is a weighted least-squares fit to the data, and uncertainties are as in Fig. ii.2.

EoR0 field as described in $\S 4.1$ of Offringa et al. (2016), the second term is the additional uncertainty in rescaling the integrated flux density measurements, and the third term is a standard error given to flux density measurements of extended sources.

\section{\$ii.1.3.3. Spectral indices and source sizes}

Spectral indices of sources are calculated in one of two ways, depending on the number of available measurements. The first method involves approximating a straight line between two flux density measurements at different frequencies, e.g. 168 and $1400 \mathrm{MHz}$, which gives an estimation of the spectral characteristics in that frequency range. A more robust method requires measurements at more than two frequencies. In these cases the spectral index is estimated by weighted least squares fitting to the flux and frequency data in logarithm space, hence fitting a power law to the spectra. We follow fitting recipes present in Hogg et al. (2010), and extend these to be unbiased in estimating the uncertainty in the fitting parameters (see Wolberg, 2006, pp. 50-51 and references therein). While the spectral energy distribution of many astronomical sources (see e.g. Jaffe and Perola 1973, Tribble 1993, Callingham et al. 2017) are not typically well described by a simple power law, over the frequency range here $(74-1400 \mathrm{MHz})$ haloes and relics tend not to show any turnovers or breaks typically do not deviate from the assumed power law 
except in rare instances (e.g. the relic in Abell 2443 which has a break near 325 $\mathrm{MHz}$ reported by Cohen and Clarke, 2011).

In parts of this work we estimate limits to flux densities where no flux, above the rms noise, is seen in an image. In particular, we use this for estimating $1.4 \mathrm{GHz}$ and $147.5 \mathrm{MHz}$ limits when $168 \mathrm{MHz}$ emission has no counterpart in the NVSS or TGSS survey images, respectively. These are used then to impose limits on the spectral indices. For such sources, we estimate the source area at $168 \mathrm{MHz}$, which is a function of the MWA beam at $B_{\text {maj }} \approx 2.3$ arcmin, and attempt to correct for the difference in beam sizes between the VLA (NVSS), GMRT (TGSS), and MWA (EoR0) by naively taking the ratios of $B_{\text {maj }}$ and correcting the area based on this ratio. The limit is then

$$
S_{\text {limit }}=\sigma_{\mathrm{rms}} f A_{168} \times \frac{4 \ln 2}{\pi B_{\mathrm{maj}} B_{\min }} \quad[\mathrm{Jy}]
$$

where $f=B_{\text {maj }} / B_{\text {maj,168 }}$ and $A_{168}$ is the source area measured at $168 \mathrm{MHz}$.

The in-house PYTHON code used also calculates the largest angular scale (LAS) of a source by comparing the angular separation between boundary pixels of a source. This is only possible for sources that are discrete and show no blending. For such sources, we estimate an angular size by making an assumption on how far the diffuse source blends into any nearby point sources. The size characterisation is important to determine if the detection is truly extended. For a non-blended source to be considered extended in this work it must have an LAS that is greater than $1.5 B_{\text {maj }}$, where $B_{\text {maj }} \approx 2.3$ arcmin, which is approximately the expected $B_{\text {maj }}$ of the EoR0 field. 


\section{§ii.2. Results from the EoR0 field}

\section{§ii.2.1. Diffuse cluster emission at $168 \mathrm{MHz}$}

Here we present the diffuse emission detected in the EoR0 field from the ACO, PSZ1 and MCXC catalogues. We detect 34 objects of interest, of which 29 are likely relics, phoenices, or haloes associated with 25 clusters. We find clusters lie within the mass range $1 \times 10^{14} \mathrm{M}_{\odot}<M_{500}<12 \times 10^{14} \mathrm{M}_{\odot}$ for those with measured masses, though those without measured masses may lie below this range, but not likely above it. The detection rate for such emission within the EoR0 field is $\sim 6.4$ per cent, which on average is lower than previous surveys, (e.g. $~ 32$ per cent: Venturi et al. 2007, 2008, 17 per cent: Bernardi et al. 2016, 12 per cent: Shakouri et al. 2016), however as mentioned, previous surveys target the most massive, and X-ray luminous clusters. Included are previously detected relics in Abell 0013, Abell 0085, and Abell 2744 (Slee and Reynolds, 1984; Slee et al., 2001; Govoni et al., 2001), phoenices in Abell 0133 and Abell 4038 (Slee and Reynolds, 1984; Slee and Roy, 1998; Slee et al., 2001), haloes in Abell 2744 and MACS J2243.3-0935 (Govoni et al., 2001; Cantwell et al., 2016), as well as the large, ambiguous emission seen in Abell 0133 (Randall et al., 2010). Included are also 5 radio galaxies on first pass thought to be possible relic, phoenix, or halo candidates. For the purpose of distinguishing between relics and phoenices, we place a limit of $400 \mathrm{kpc}$ as a maximum size of phoenix. Where emission scale approaches this size we look at the spectral index and location, where a linear size approaching $400 \mathrm{kpc}$ with ultra-steep spectral indices $(\alpha<-1.5$, Kempner et al. 2004) and a location closer to the cluster's centre would be suggestive of phoenices rather than relics. Table ii.2 summarises the results of the diffuse emission search. Following this, §ii.2.2 describes each cluster along with the diffuse emission detected within it. Images featuring optical backgrounds are three-colour images with red, green, and blue corresponding to infrared, red, and blue respectively, using data from the DSS2, unless otherwise stated in the corresponding caption. For this section, a flat $\Lambda$ CDM cosmology is adopted with $H_{0}=68 \mathrm{~km} \mathrm{~s}^{-1} \mathrm{Mpc}^{-1}$, $\Omega_{m}=0.3$, and $\Omega_{\Lambda}=0.7$, used to convert angular scales to linear with a given redshift. Note that for all radio, X-ray, and optical images in this work: north is up and east is left unless otherwise stated. 


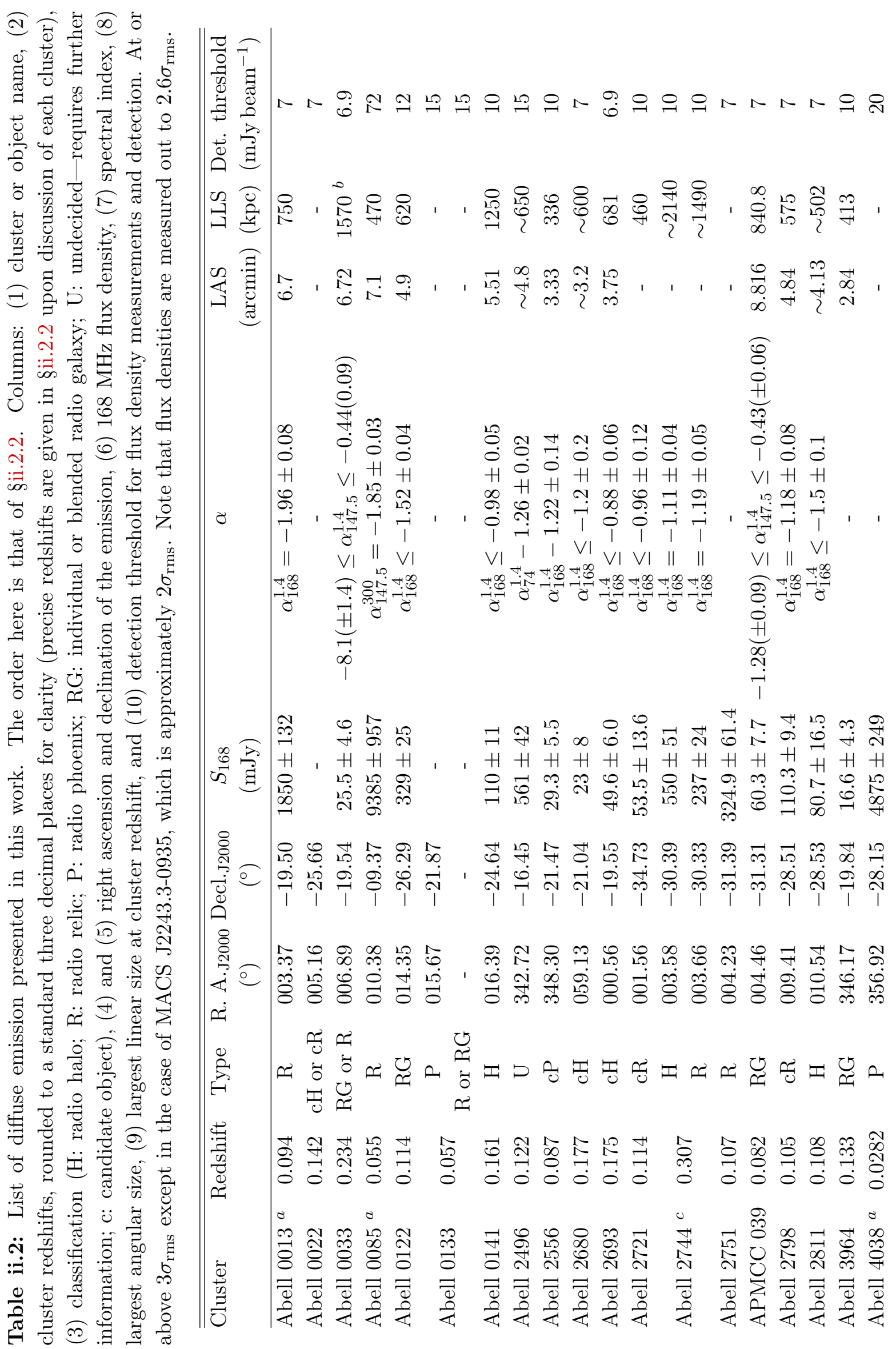




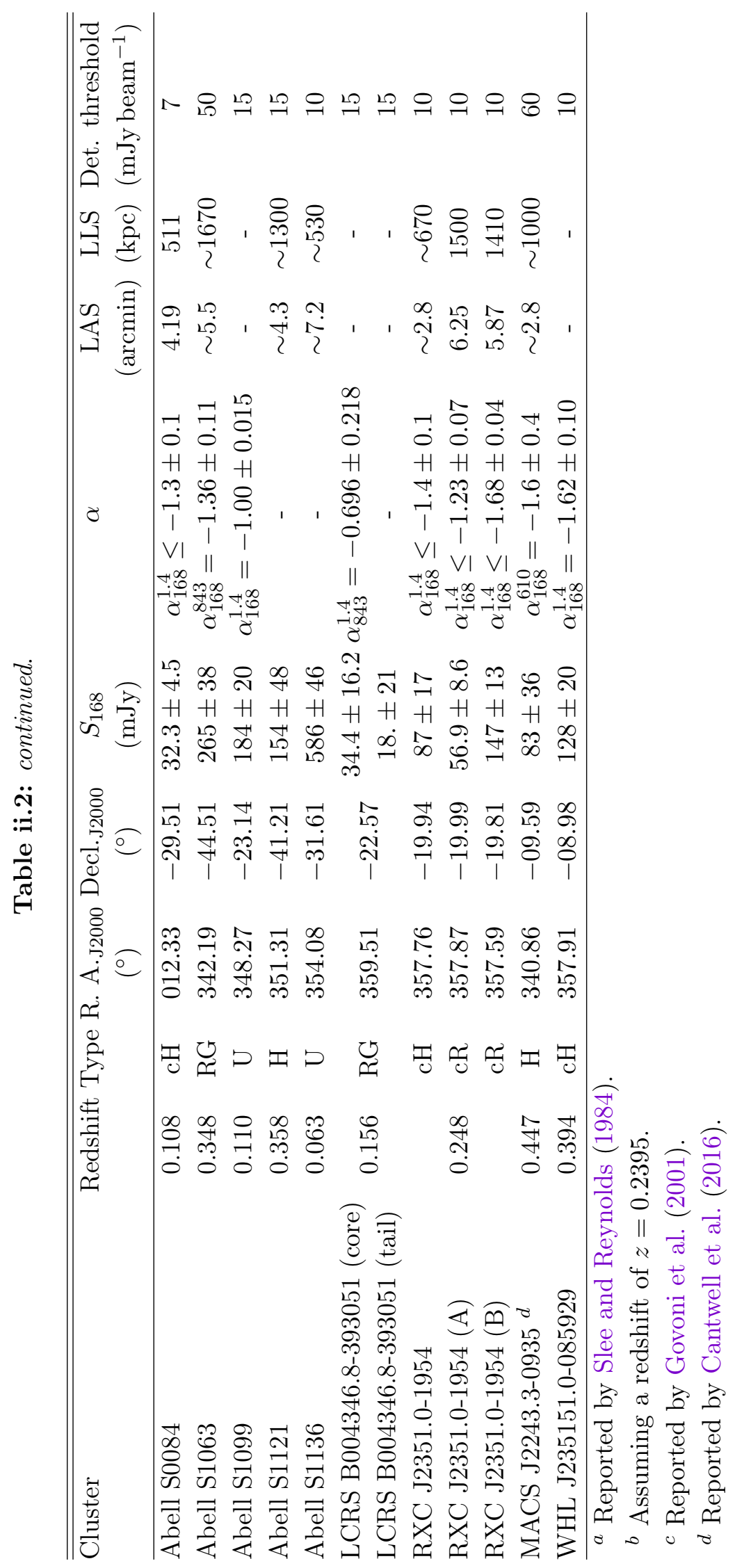




\title{
§ii.2.2. Individual galaxy clusters
}

\author{
§ii.2.2.1. Abell 0013
}

The galaxy cluster Abell 0013 (MCXC J0013.6-1930; PSZ1 G072.48-78.46) has mass $M_{\mathrm{YZ}, 500}=2.79_{-0.38}^{+0.36} \times 10^{14} \mathrm{M}_{\odot}(\mathrm{PSZ} 1)$, a redshift of $z=0.0940$, and X-ray luminosity $L_{500}=1.236332 \times 10^{37} \mathrm{~W}(\mathrm{MCXC})$. Slee and Reynolds (1984) report the detection of a steep-spectrum radio relic after $1.465 \mathrm{GHz}$ and $4.885 \mathrm{GHz}$ VLA imaging. Slee et al. (2001) provide further VLA observations at $1.425 \mathrm{GHz}$ and show filamentary structure in the emission. The top panel of Fig. ii.4 shows an RGB image with MWA, NVSS, and TGSS contours overlaid. The steep spectrum relic is recovered in the MWA image, labelled "A" in the figure, and extends beyond what is expected due to the difference in resolution. The flux density at $168 \mathrm{MHz}$ is measured to be $S_{168}=1.850 \pm 0.132 \mathrm{Jy}$, with an LAS of 6.7 arcmin and largest linear scale (LLS) at the cluster's redshift of $750 \mathrm{kpc}$. Within the NVSS catalogue the source is split into two components: NVSS J001332-193023 and NVSS J001326192950. These sources are reported to have flux densities of $10.7 \pm 2.1 \mathrm{mJy}$ and $18.0 \pm 2.0$ mJy respectively (Condon et al., 1998). With comparison to Fig. 2 of Slee et al. (2001) we conclude that both of these NVSS sources are a single source and so add them to arrive at a flux density of $S_{1.4}=28.7 \pm 4.1 \mathrm{mJy}$ for the emission. We calculate a spectral index between the $1.4 \mathrm{GHz}$ and $168 \mathrm{MHz}$ measurements as $\alpha_{168}^{1.4}=-1.96 \pm 0.08$. Such a steep spectral index, along with apparent proximity to the cluster's core is suggestive of a radio phoenix, however at the projected size of $750 \mathrm{kpc}$ this becomes too large for small-scale turbulence to re-accelerate electrons. This size is indicative of a radio relic. It is entirely possible the relic is further on the periphery, only appearing close to the centre due to projection effects. Slee et al. (2001) report the spectral index between 1.465 $\mathrm{GHz}$ and $1.385 \mathrm{GHz}$ to be $\alpha_{1.385}^{1.465}=-4.4 \pm 0.4$. Due to the resolution of the these observations, it is likely the relic has not been detected in its entirety, resulting in flux being resolved out of the VLA images and resulting in an overly steep spectral index. Slee et al. provide high resolution $1.4 \mathrm{GHz}$ analyses of the relic, showing the structure that is traced by the TGSS and NVSS emission as in the top panel of Fig. ii.4. They consider the possibility of the diffuse emission being old lobes of one of the optically visible cluster members near the core. They identify the most probable host as the brightest cluster galaxies (BCG) 2MASX J001334011929017 and 2MASX J00133853-1930007 with redshifts $z=0.099272 \pm 0.000217$ and $z=0.090529 \pm 0.000157$ (Quintana and Ramirez, 1995) respectively.

The bottom panel of Fig. ii.4 shows exposure corrected, smoothed XMMNewton data (Obs. ID 0200270101, PI Ehle). The bulk of the central radio emission is offset from the main component of the X-ray plasma as well as the optical concentration of galaxies. The offset from the X-ray and optical cores suggests that the emission is not a cluster halo. In addition to the relic " $\mathrm{A}$ ", there is extended emission seen (marked "B" in Fig. ii.4) that has no $1.4 \mathrm{~Hz}$ or $147.5 \mathrm{MHz}$ counterpart. The extension may be related to the nearby point-source, NVSS J001408-193218 

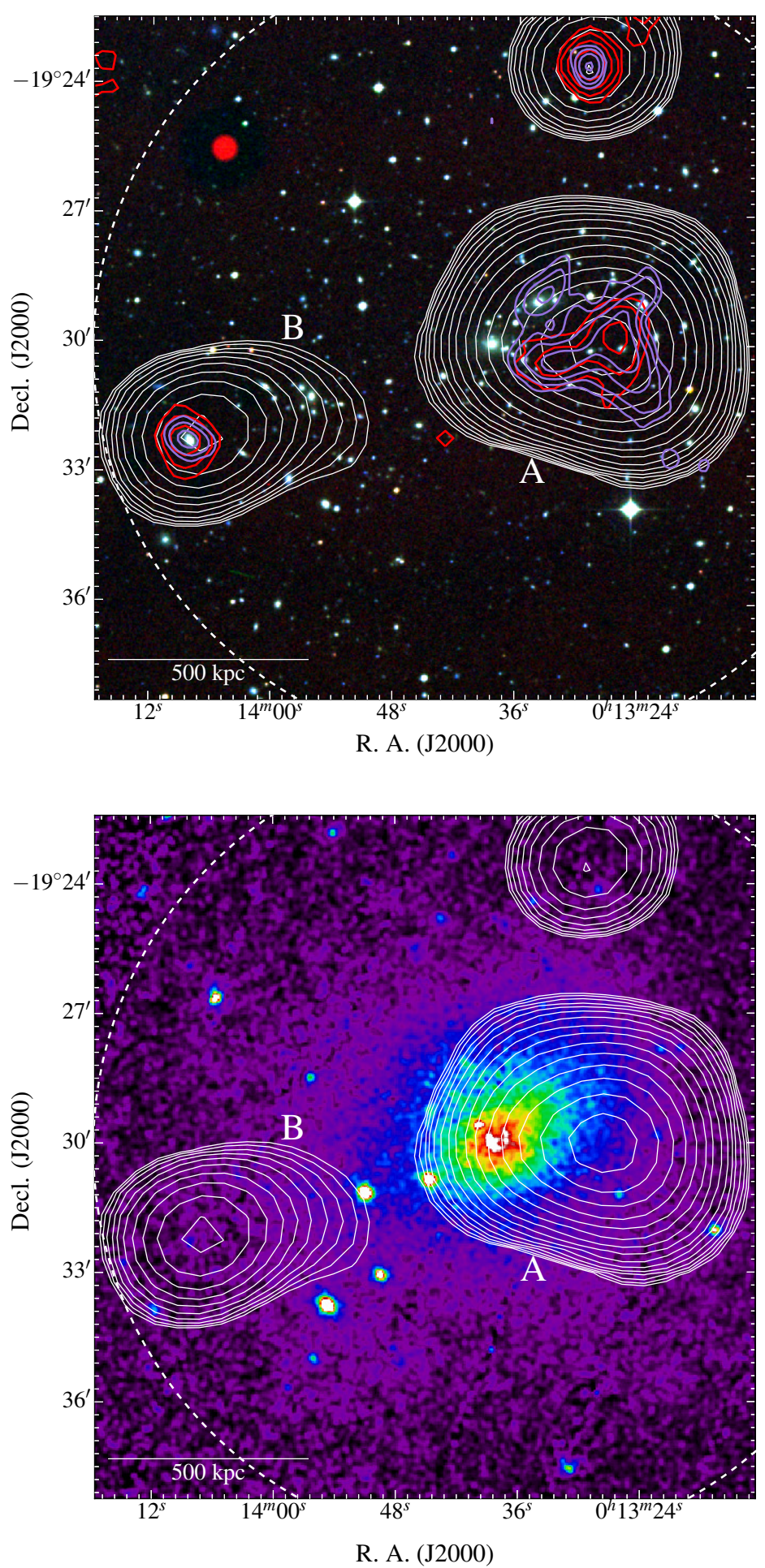

Figure ii.4: Abell 0013. Top: RGB image with contours overlaid as follows: EoR0, white, beginning at $7 \mathrm{mJy}_{\text {beam }}{ }^{-1}$ and increasing by a factor of $\sqrt{2}$; NVSS, red, beginning at $1.5 \mathrm{mJy}_{\text {beam }}{ }^{-1}$ and increasing by a factor of 2 ; TGSS, medium purple, beginning at $13.2 \mathrm{mJy}_{\text {beam }}{ }^{-1}$ also increasing by a factor of 2 . A factor of 2 is chosen here so that optical galaxies remain unobscured by radio contours. The dashed circle is centred on Abell 0013 with a radius of 1 Mpc. Bottom: XMM-Newton X-ray background with EoR0 field contours alongside X-ray contours. 
(2MASX J00140813-1932176) with clear optical ID. However, we the extension seems to trace the optically visible subgroup and may be emission associated with it. This emission may be an example of diffuse emission tracing the accretion shock of an in-falling galaxy group. Shakouri et al. (2016) consider emission associated with the clusters RXC J0225.1-2918 and Abell S0258 as a possible example of this, though even with their polarimetry and higher resolution they are unable to confirm this and we are unable to do so here as well.

This radio relic (and cluster in general) is a good example of the power of the MWA for finding steep-spectrum sources and using higher resolution imaging to properly ascertain their nature. Without auxiliary data we run into issues of classification with the MWA alone, where we know steep-spectrum diffuse cluster emission exists but are unable to definitively classify the emission as either a halo, relic, or otherwise, without further data. Conversely, with only the high-resolution data diffuse flux is resolved out. In the case of Abell 0013, one might argue it shows characteristics of a radio phoenix in its steep spectral index and location near the centre of the cluster (unless its location is being projected onto the central region). However, its newly detected linear size goes against this classification due to the shock and turbulence requirements now becoming cluster-wide rather than local to the emission.

\section{§ii.2.2.2. Abell 0022}

The cluster Abell 0022 (MCXC J0020.7-2542; PSZ1 G042.77-82.97) has a redshift of $z=0.142352 \pm 0.000327$ (Pimbblet et al., 2006), mass $M_{\mathrm{YZ}, 500}=4.56_{-0.44}^{+0.42} \times 10^{14}$ $\mathrm{M}_{\odot}(\mathrm{PSZ} 1)$, and X-ray luminosity $L_{500}=2.872451 \times 10^{37} \mathrm{~W}(\mathrm{MCXC})$. The cluster features extremely diffuse, faint emission that appears to permeate the cluster. Fig. ii.5 shows the emission extending from the centre of the cluster northward. Although we see from the NVSS and TGSS data that the MWA emission is coincident with three point sources: NVSS J002042-254239, associated with a member of the intervening galaxy triple DUKST 473-042; NVSS J002048254437; and NVSS J002058-253957, emission associated with the cluster member 2MASX J00205811-2539516. The MWA data extends considerably further north reminiscent of the cluster halo in Abell 3888 (Shakouri et al., 2016). We do not obtain a flux density measurement for the extended emission as it blends into the emission from 2MASX J00205811-2539516, which has a flux density contribution that we are unable to subtract as there is neither significant emission seen at 1.4 $\mathrm{GHz}$ in the NVSS image, nor in the $147.5 \mathrm{MHz}$ emission in the TGSS image.

XMM-Newton data is shown in the bottom panel of Fig. ii.5 (Obs. ID 0201900301, PI Böhringer), which were taken and reduced as part of the REXCESS survey (Böhringer et al., 2007; Pratt et al., 2009). The $168 \mathrm{MHz}$ radio emission extends far beyond the X-ray emission, however it is likely the bulk of the halo sits coincident with the X-ray peak, but is blending with DUKST 473-042, which has a spectroscopic redshift of $z=0.063821 \pm 0.000500$ (Ratcliffe et al., 1998) and is seen in the centre of the field. We cannot unambiguously classify the extended emission 

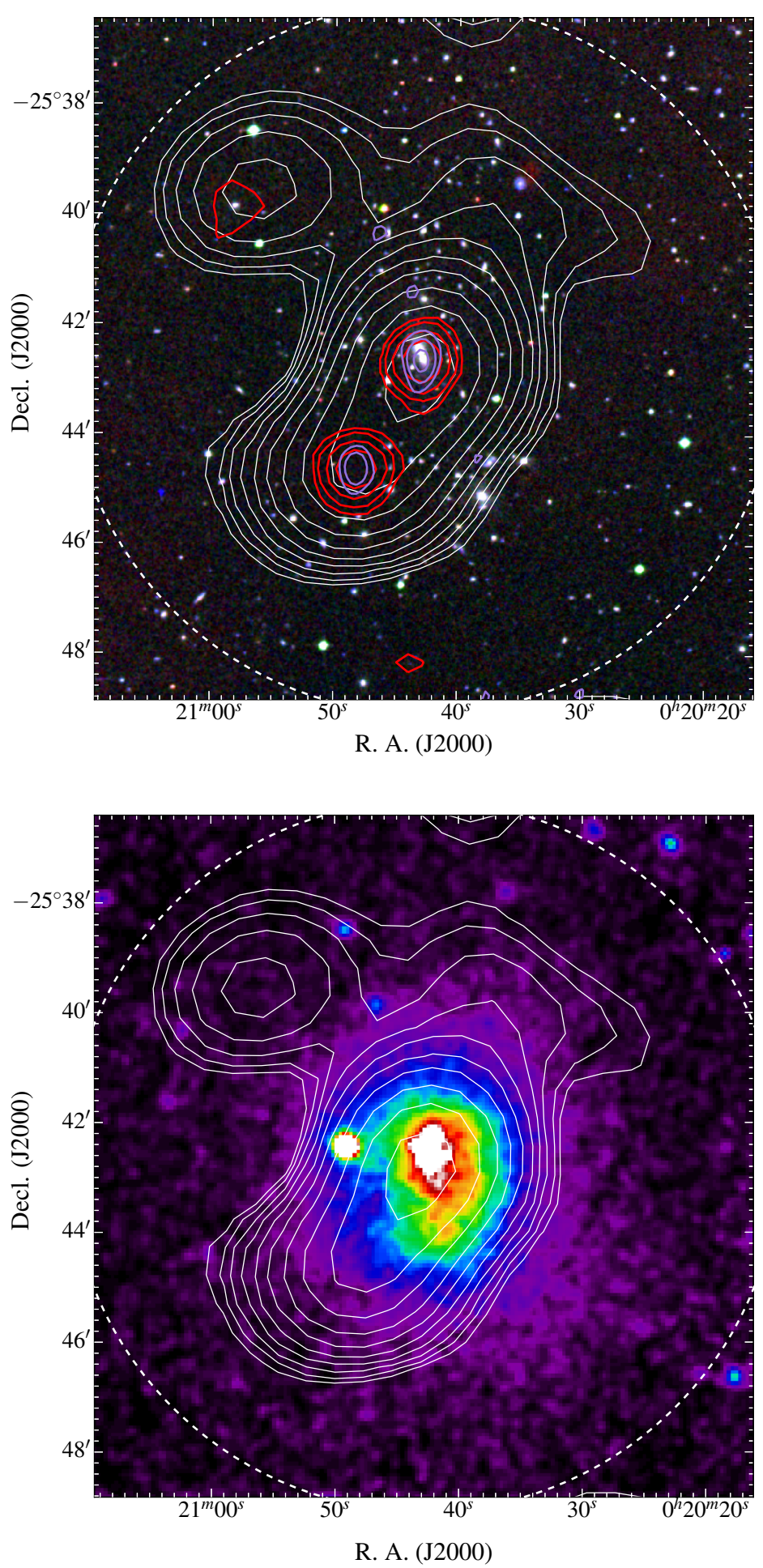

Figure ii.5: Faint candidate halo in Abell 0022. Top: RGB image with contours overlaid as follows: EoR0, white, beginning at $7 \mathrm{mJy}_{\text {beam }}{ }^{-1}$ increasing by a factor of $\sqrt{2}$; NVSS, red, beginning at $1.5 \mathrm{mJy}_{\text {beam }}{ }^{-1}$ increasing by a factor of 2 ; TGSS, medium purple, beginning at $21 \mathrm{mJy}_{\text {beam }}^{-1}$ also increasing by a factor of 2 . The dashed circle is centred on the cluster with a radius of $1 \mathrm{Mpc}$. Bottom: X-ray image from the REXCESS survey with EoR0 contours overlaid as in the top panel along with X-ray contours increasing with a factor of 2 in black. 
as either a candidate halo, relic, or both. We suggest follow-up observations at a higher resolution to disentangle the point sources from the diffuse radio emission.

\section{§ii.2.2.3. Abell 0033}

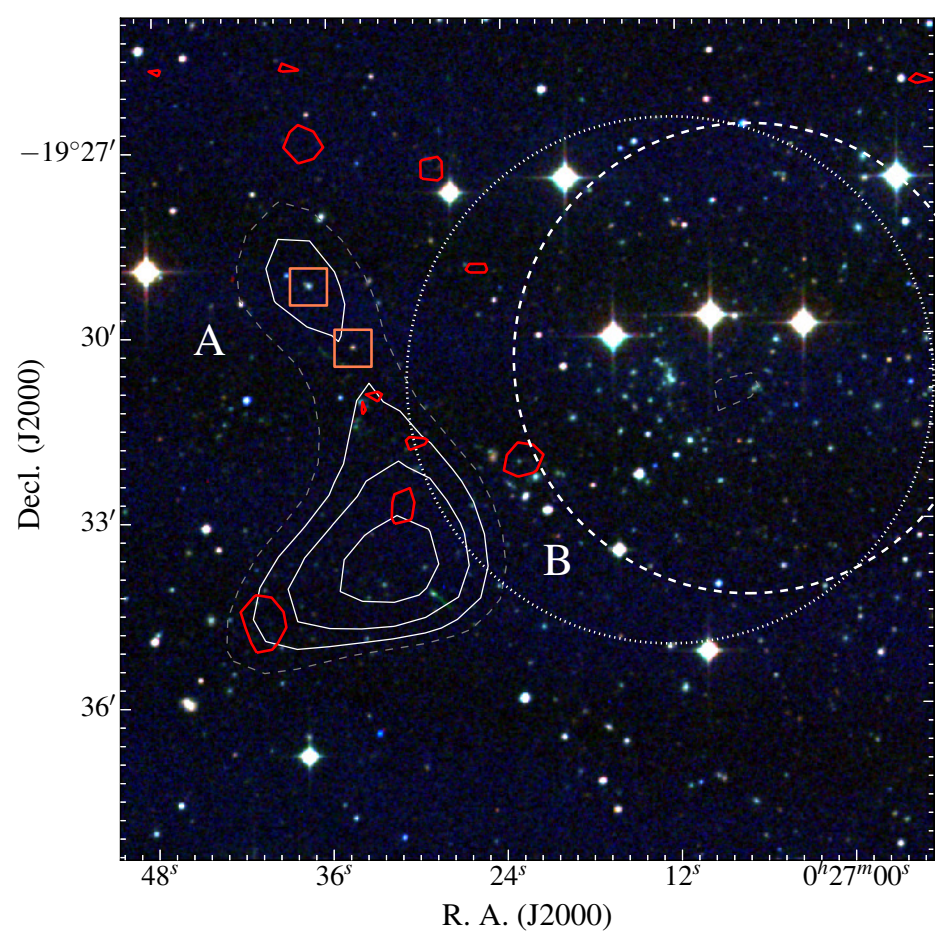

Figure ii.6: Candidate relic on the periphery of Abell 0033. The background is an RGB image with contours overlaid as follows: EoR0, white, $3 \sigma_{\mathrm{rms}}$ beginning at $6.9 \mathrm{mJy}^{-1} \mathrm{beam}^{-1}$ increasing by a factor of $\sqrt{2}$ and grey, dashed, $2 \sigma_{\mathrm{rms}}$ at 4.6 $\mathrm{mJy}_{\text {beam }}{ }^{-1}$; NVSS, red, beginning at $1.5 \mathrm{mJy}_{\text {beam }}{ }^{-1}$ increasing by a factor of 2 . No TGSS emission is seen above the $3 \sigma_{\mathrm{rms}}$ level of $25.8 \mathrm{mJy}_{\text {beam }}{ }^{-1}$. The dashed circle is centre on the position of Abell 0033 and the dotted circled is centred on WHL J002712.5-193045, both with radii Mpc at the reported redshifts. They are suspected to be the same cluster (see main text). The boxes indicate possible optical IDs for the diffuse emission.

Fig. ii.6 shows curious emission on the periphery of both Abell $0033(z=$ 0.28, photometric; Leir and van den Bergh 1977) and WHL J002712.5-193045 ( $z=$ 0.2395, spectroscopic; Wen and Han 2013). The white circles in Fig. ii.6 have 1 Mpc radii about the cluster centres. The two clusters are separated by an angular distance of $\sim 80$ arcsec, and given the clear concentration of optical galaxies seen in the DSS2 images, they are likely the same cluster and we hereafter consider there to be only Abell 0033 at the redshift of $z=0.2395$. The grey, dashed contour in Fig. ii. 6 is at the $2 \sigma_{\text {rms }}$ level to indicate the possibility of the two objects, Obj. $\mathrm{A}$ and $\mathrm{B}$, being a single piece of extended emission on the cluster periphery. If this is the case, the entire structure has a flux density of $S_{168}=25.5 \pm 4.6 \mathrm{mJy}$, and an LAS is 6.72 arcmin which translates to an LLS of $1570 \mathrm{kpc}$ at $z=0.2395$. 
Both the NVSS and TGSS surveys do not show significant emission within the area of the $168 \mathrm{MHz}$ emission. From this we provide upper limits on the $1.4 \mathrm{GHz}$ and $147.5 \mathrm{MHz}$ flux densities of $S_{1.4} \leq 10 \mathrm{mJy}$ and $S_{147.5} \leq 73 \mathrm{mJy}$. This gives a limit on the spectral index between $147.5 \mathrm{MHz}$ and $1.4 \mathrm{GHz}$ of $-8.1( \pm 1.4) \leq \alpha \leq$ $-0.44( \pm 0.09)$, assuming a simple power law to the SED. This range of spectral indices is consistent with either typical radio galaxies of $\alpha=-0.8$ (Condon, 1992), the steep spectrum cluster relics (e.g. de Gasperin et al., 2014), or the dying radio galaxies often found within clusters (e.g. Murgia et al., 2011). However the lowerlimit covers a range that is unrealistic for known astronomical sources so is not definitive for classification purposes or useful in any way.

Upon inspection of the optical data, two possible optical IDs are found and highlighted with boxes in Fig. ii.6. There is an optically visible source reported in the USNO-A2.0 catalogue, USNO-A2.0 0675-00178746, sitting between A and B. The RGB image of Fig. ii.6 shows significant reddening of this optical source, consistent with a high redshift. The shape of the emission is then reminiscent of the lobes of a radio galaxy and the overall morphology is similar to the dead radio galaxy associated with the lenticular galaxy NGC 1534-recently discovered using the MWA (Hurley-Walker et al., 2015) —with faint lobes and steep spectral indices as ancient remnants of old episodes of AGN activity. However, Obj. A has an optically visible galaxy, GALEXASC J002737.37-192909.3, near its centre and so could be associated with it. In this case, Obj. B is unlikely to be associated with Obj. A and may be a cluster radio relic. We offer these possibilities as the origins of this emission, but cannot classify the emission definitively. Note that the LLS presented in Table ii.2 assumes Obj. A and B are a single source and associated with the cluster with a redshift of $z=0.2395$.

\section{§ii.2.2.4. Abell 0085}

Abell 0085 (MCXC J0041.8-0918; PSZ1 G115.20-72.07) is a nearby cluster with a redshift of $z=0.055061 \pm 0.000340$ (Oegerle and Hill, 2001), mass $M_{\mathrm{YZ}, 500}=$ $4.90_{-0.22}^{+0.21} \times 10^{14} \mathrm{M}_{\odot}(\mathrm{PSZ} 1)$ and X-ray luminosity $L_{500}=5.100085 \times 10^{37} \mathrm{~W}(\mathrm{MCXC})$. Slee and Reynolds (1984) report the detection of a phoenix offset from the cluster centre, and Giovannini and Feretti (2000) provide follow-up $300 \mathrm{MHz}$ imaging with the VLA and ascertain an LLS for the radio phoenix of $386 \mathrm{kpc}$ (corrected for this cosmology). $168 \mathrm{MHz}$ emission coincides with the previously detected phoenix (labelled "A" in Fig. ii.7), and has an approximate LLS of $470 \mathrm{kpc}$. The in-house PYTHON script does not deal with blended emission. To obtain the flux density of the steep spectrum emission in Abell 0085 we use AEGEAN with an rms noise of $24 \mathrm{mJy}_{\text {beam }}{ }^{-1}$, seedclip of 3, and floodclip of 2, The flux density is measured to be $S_{168}=9.385 \pm 0.957 \mathrm{Jy}$. $1.4 \mathrm{GHz}$ emission from the NVSS traces the relic as described by Slee et al. The TGSS shows $147.5 \mathrm{MHz}$ emission beyond that of the NVSS despite similar resolutions with an extended structure to the southeast, tracing the emission at $300 \mathrm{MHz}$ shown by Giovannini and Feretti. This extension is also encompassed by the $168 \mathrm{MHz}$ emission. We do not use 

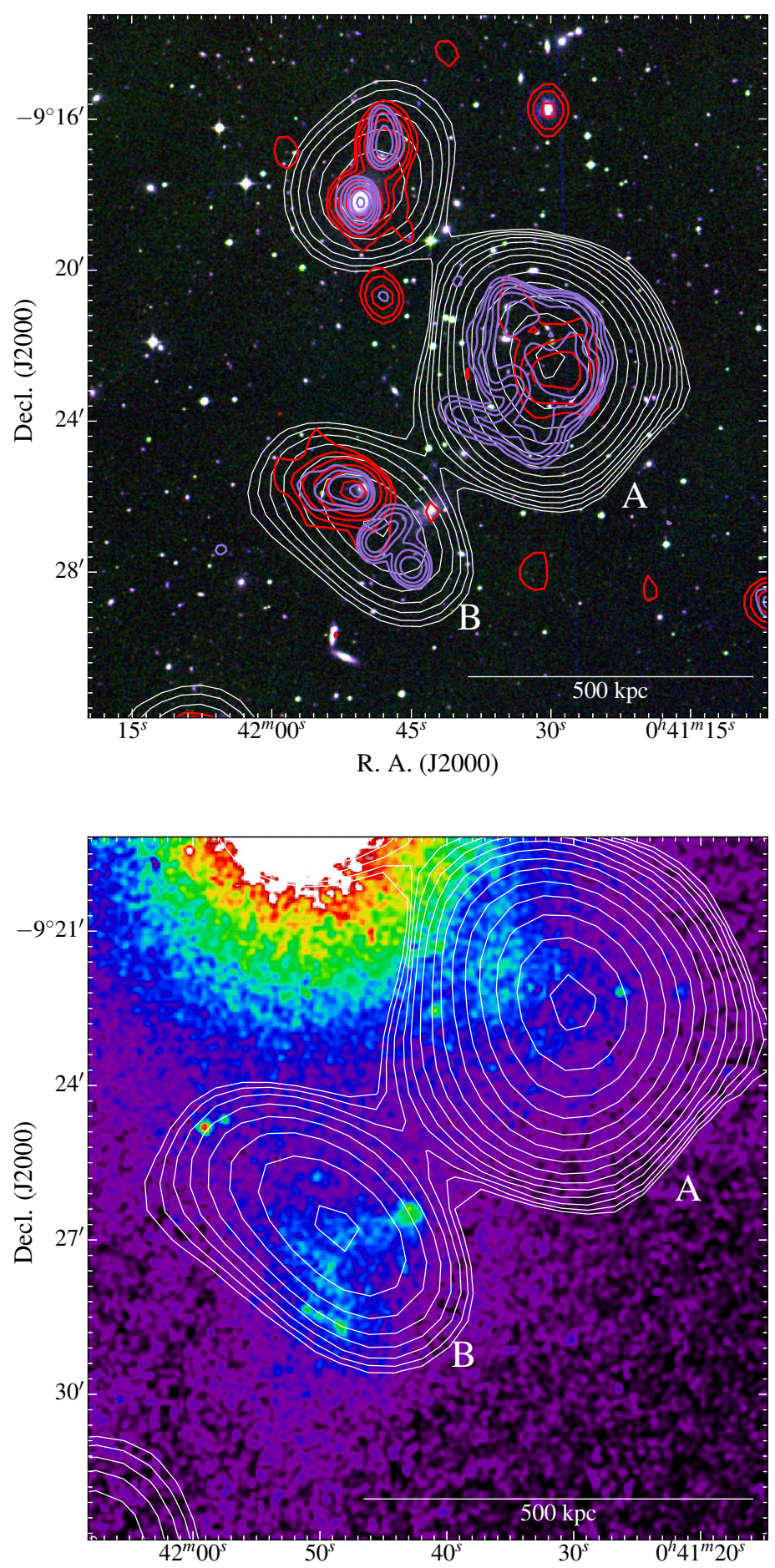

R. A. (J2000)

Figure ii.7: Abell 0085. Top: The background is an RGB image with contours overlaid as follows: EoR0, white, beginning at $49.7 \mathrm{mJy}_{\text {beam }}{ }^{-1}$ increasing by a factor of increasing by $\sqrt{2}$; NVSS, red, beginning at $1.5 \mathrm{mJy}_{\text {beam }}{ }^{-1}$ increasing by a factor of 2; TGSS, medium purple, beginning at $9.6 \mathrm{mJy}_{\text {beam }}^{-1}$ also increasing by factor of 2. Bottom: XMM-Newton image with EoR0 contours overlaid as in the top panel. Both panels show the linear scale at the cluster's redshift. 
the $1.4 \mathrm{GHz}$ flux density from the NVSS as a large portion of the relic is undetected. Rather, we use the $147.5 \mathrm{MHz}$ TGSS image and measure a flux density of $S_{147.5}=10.21 \pm 0.07 \mathrm{Jy}$. We use the $300 \mathrm{MHz}$ measurement of $S_{300}=2.739$ Jy (Giovannini and Feretti, 2000) though include a 10 per cent uncertainty as no uncertainty is quoted by Giovannini and Feretti. Thus, we calculate a spectral index of $\alpha_{147.5}^{300}=-1.85 \pm 0.03$, though note that the TGSS image is likely missing flux due to resolution and missing baselines, which suggests the relic may have an even steeper spectral index.

The radio source to the southeast of the relic (labelled "B" in Fig. ii.7) has extended $168 \mathrm{MHz}$ emission beyond the source seen in the NVSS which is likely associated with the galaxy SDSS J004150.17-092547.4. The TGSS 147.5 MHz data shows two distinct sources within this extended, steep-spectrum emission. The bottom panel of Fig. ii.7 shows a zoomed-in view of Obj. B, with MWA contours overlaid on exposure corrected, smoothed XMM-Newton data (Obs. ID 0723802101, PI de Plaa). Obj. B features an extension to the bulk of the X-ray emitting plasma at the cluster's core. Kempner et al. (2002) suggest that this extension of X-ray emission, along with the complex of radio sources Obj. B, is representative of subcluster asymmetrically merging with the main cluster of Abell 0085 .

\section{§ii.2.2.5. Abell 0122}

Abell 0122 (MCXC J0057.4-2616), with a redshift of $z=0.113478$ (Zaritsky et al., 2006), mass $M_{\mathrm{X}, 500}=1.7267 \times 10^{14} \mathrm{M}_{\odot}$, and X-ray luminosity $L_{500}=0.861163 \times$ $10^{37} \mathrm{~W}(\mathrm{MCXC})$, features a strong diffuse source at its centre. This diffuse emission has a flux density of $S_{168}=329 \pm 25$ mJy with an LAS of 4.9 arcmin. At the redshift of the cluster the emission has a projected LLS of $620 \mathrm{kpc}$. There is no significant $1.4 \mathrm{GHz}$ emission seen with the NVSS survey, though the $147.5 \mathrm{MHz}$ TGSS data shows extended emission. The TGSS ADR1 catalogue splits this into two distinct sources with its higher $7 \sigma_{\text {rms }}$ cutoff for source detections, compared to Fig ii. 8 which has TGSS contours at the $3 \sigma_{\text {rms }}$ level typically necessary for faint diffuse emission detections. We provide a $1.4 \mathrm{GHz}$ flux limit of $S_{1.4} \leq 13 \mathrm{mJy}$ and a corresponding spectral index of $\alpha_{168}^{1.4} \leq-1.52 \pm 0.04$.

The bottom panel of Fig: ii.8 shows exposure corrected, smoothed XMMNewton data (Obs. ID 0504160101, PI Sivanandam). The $168 \mathrm{MHz}$ radio emission fills the X-ray plasma as is typical of cluster radio haloes and the location, steep spectral index, and X-ray emission are suggestive of an ultra-steep spectrum radio halo (USSRH; see e.g. Cassano et al., 2006). However the 147.5 MHz morphology appears to suggest a tailed radio galaxy, or other radio galaxy-related origin. The tailed radio galaxy origin is somewhat hampered by the lack of optical ID coincident with the peak of the $147.5 \mathrm{MHz}$ emission. The BCG, 2MASX J005722882616528 , lies offset from the peak by approximately 10 arcsec. Given the morphology, steep spectral index, and displacement of an optical host, there exists the possibility that the emission is that of an old, dead radio galaxy, likely associated 

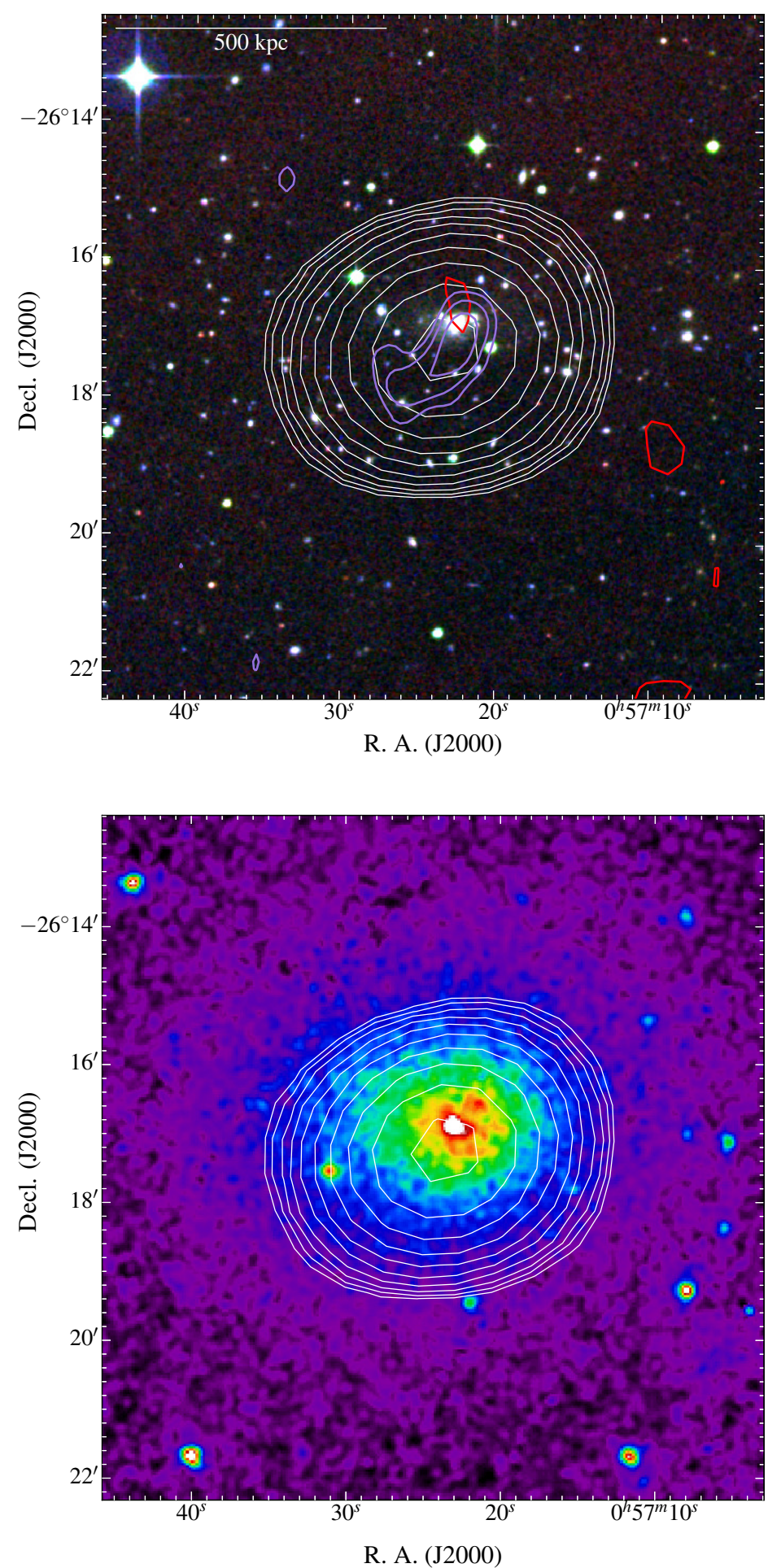

Figure ii.8: Steep-spectrum emission at the centre of Abell 0122. Top: The background is an RGB image with contours overlaid as follows: EoR0, white, beginning at $12 \mathrm{mJy}$ beam $^{-1}$ and increasing by a factor of $\sqrt{2}$; NVSS, red, beginning at 1.5 mJy and increasing by a factor of 2; TGSS, medium purple, beginning at $13.5 \mathrm{mJy}$ also increasing by a factor of 2 . The linear sale is at the cluster's redshift. Bottom: The background image is archival XMM-Newton data with EoR0 contours as in the top panel. The dashed circle is centred on Abell 0122, and has a radius of $1 \mathrm{Mpc}$. 
with the BCG. Abell 0122 shows no evidence in the either X-ray emission or the optical density that would suggest the cluster is undergoing, or had undergone, a merger event. If the turbulent re-acceleration model is correct, a halo in this cluster of this power would be unusual. Given the morphology of the source in the TGSS image, we have classified this emission as a radio galaxy, though note that there is the possibility the emission represents a mini-halo due to the morphologically relaxed nature of the X-ray plasma.

§ii.2.2.6. Abell 0133

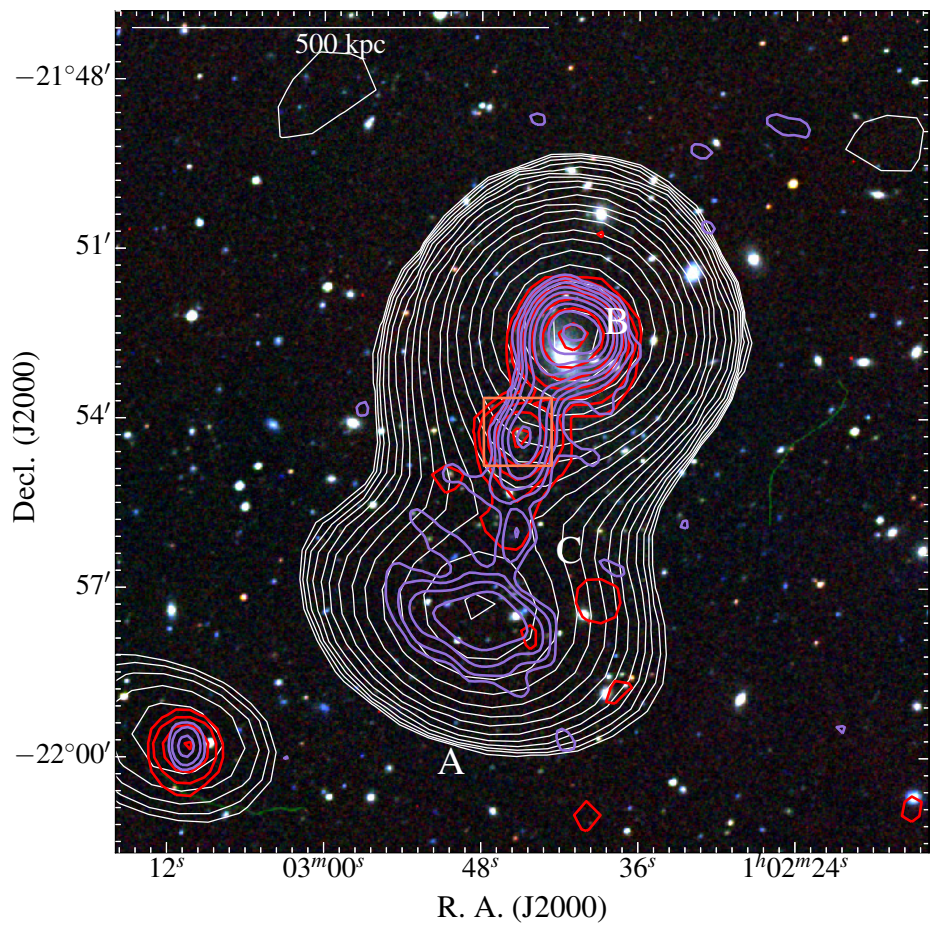

Figure ii.9: The centre of Abell 0133. The background is an RGB image with contours overlaid as follows: EoR0, white, beginning at 15 mJy beam $^{-1}$ increasing with a factor of $\sqrt{2}$; NVSS, red, beginning at $1.5 \mathrm{mJy}_{\text {beam }}^{-1}$ and increasing with a factor of 2; TGSS, medium purple, $13.2 \mathrm{mJy}_{\text {beam }}{ }^{-1}$ also increasing with a factor of 2. The linear scale is at the redshift of the cluster, and Obj. A is the diffuse emission of interest.

Abell 0133 (MCXC J0102.7-2152; PSZ1 G149.55-84.16) has a redshift of $z=$ 0.0562 (Way et al., 1997), X-ray luminosity $L_{\mathrm{X}, 500}=1.460216 \times 10^{37} \mathrm{~W}(\mathrm{MCXC})$, and mass $M_{\mathrm{YZ}, 500}=3.08_{-0.24}^{+0.23} \times 10^{14} \mathrm{M}_{\odot}(\mathrm{PSZ1})$. The cluster has been studied extensively in X-ray (e.g. Reichert et al., 1981; Fujita et al., 2002, 2004) along with the multi-wavelength study by Randall et al. (2010) which all point towards the disturbed, dynamic nature of the the cluster. Further, steep-spectrum radio emission in the form of a radio phoenix was detected by Slee and Reynolds (1984) 30 arcsec from the cD, ESO 541-G013. Further high-resolution follow-up observations by Slee et al. (2001) showed filamentary structure to this phoenix. An important 
result of the X-ray studies show that the X-ray-emitting gas is disturbed, with the possibility of a weak shock (Fujita et al., 2004) creating a tongue-like feature pointing to the northwest. This feature coincides with the phoenix reported by Slee and Reynolds (1984).

Fig. ii.9 shows the cluster centre with the emission of interest, with Obj. A a large, possible lobe, Obj. B the radio phoenix, and the orange square indicating the possible ID for double-lobe-like structure, along with Obj. C, an interesting knot. Randall et al. (2010) discuss the possibility of the entire structure representing a giant, background radio galaxy, with the core and host marked in Fig. ii.9. As part of this interpretation, the phoenix is thought to be a separate entity, most likely associated with the cluster. We consider an alternative explanation not covered by Randall et al. (2010) where the southern lobe A is in fact a relic. This explanation draws on deep radio observations of 1E 0657-56 (the Bullet Cluster; Liang et al., 2000, 2001; Shimwell et al., 2014, 2015; Srinivasan, 2015). Liang et al. (2000) show low resolution radio imaging of the Bullet Cluster, and further X-ray observations provide high resolution imaging to show the directionality of the shock (Markevitch, 2006) with clear diffuse emission located to the east of the west-ward $\mathrm{X}$-ray shock. This piece of diffuse emission is considered a relic, created through back-shock of the massive, merging system (Shimwell et al., 2014). We consider the possibility that the extended filament between the northern B and southern A lobes in Abell 0133 is in fact of similar origin. This scenario would suggest that the southern lobe is a relic similar to the bulb portion of the relic in the Bullet Cluster. The locational difference between the possible relic in Abell 0133 and relic in the Bullet Cluster could be simple projection effects. Obj. C in Fig. ii.9 marks a knot in the filament, clear in the medium purple TGSS contours, and seen in Figure 5(d) of Randall et al. (2010). This has no optical ID so is not necessarily an unassociated point source. In the case of structure being that of a background giant radio galaxy (GRG), there is a requirement that extreme twisting of the core relative to the southern lobe must have occurred, or is in the process of occurring. Whilst such disjoint motion is seen in lobed radio galaxies (e.g. NGC 326: Fanti et al., 1977; Murgia et al., 2001), these galaxies with disturbed lobes typically reside within clusters, where the ICM plays a crucial role in shaping the jets through ram pressure as the galaxy core travels through or precesses within the medium. It may be that the structure is a GRG in a background cluster. The supposed optical host has a redshift of $z=0.2930$ (2MASX J01024529-2154137: Owen et al., 1995; Slee et al., 2001; Randall et al., 2010), however there are no other available redshifts within $\sim 2000 \mathrm{~km} \mathrm{~s}^{-1}$ of $z=0.2930$ near 2MASX J01024529-2154137. We find that the $147.5 \mathrm{MHz}$ TGSS contours in Fig. ii.9 show that the peak of this emission near the core of the GRG does not align with the proposed optical ID, marked with an orange square, though the 1.4 GHz NVSS contours do align well with 2MASX J01024529-2154137. GRGs are found independent of clusters, but the ICM is required for jets to be bent from the axis of the GRG which may be the case here. 
We estimate the extent of the diffuse emission within both the relic and radio galaxy interpretations. If the emission is that of a radio galaxy, we find LAS to be 10.4 arcmin, which at $z=0.2930$ corresponds to an LLS of $2.82 \mathrm{Mpc}$ and at $z=0.0562$ an LLS of $701 \mathrm{kpc}$. In the relic scenario, we consider the southern lobe to be a relic, and measure the east-west dimensions. The LAS is found to be 6.0 arcmin, which corresponds to an LLS of $405 \mathrm{kpc}$ at the cluster's redshift. As mentioned, the filament between the core and southern lobe would likely be part of this relic in this scenario, the size of which is difficult to estimate given any additional emission from the $\mathrm{cD}$, the phoenix, as well as the intruding emission likely associated with 2MASX J01024529-2154137. We do no provide any further certainty on the nature of the emission, merely offer the alternate explanation of radio relic akin to that in the Bullet Cluster. In Table ii.2 we list the phoenix, as well as the the ambiguous emission as both a relic and a radio galaxy.

\section{\$ii.2.2.7. Abell 0141}

Abell 0141 (MCXC J0105.5-2439) is a distant cluster with a redshift of $z=0.230$ (Struble and Rood, 1999). We present a hitherto undetected radio halo at its centre coinciding with the optical mass concentration. The mass and X-ray luminosity are $M_{\mathrm{X}, 500}=4.7167 \times 10^{14} \mathrm{M}_{\odot}$ and $L_{500}=5.160525 \times 10^{37} \mathrm{~W}(\mathrm{MCXC})$. The top panel of Fig. ii.10 shows the cluster with an RGB image as a background with the $168 \mathrm{MHz}$ contours overlaid to illustrate the radio halo's location relative to the cluster.

Previous searches for diffuse radio emission in this cluster includes a search by Venturi et al. (2007) as part of the GMRT Radio Halo Survey (GRHS), in which their $610 \mathrm{MHz}$ images with $1 \sigma=90 \mathrm{\mu Jy}$ beam $^{-1}$ did not detect anything resembling the emission seen at $168 \mathrm{MHz}$. The cluster had been a curiosity due to the lack of a halo as the cluster is undergoing a merger. The bottom panel of Fig. ii.10 shows the dynamic nature clearly in the bi-modality of the X-ray plasma. Further, the optical concentration of galaxies trace the two X-ray peaks and the elongation of the X-ray emission and $168 \mathrm{MHz}$ radio emission. Dahle et al. (2002) comment on the ill-defined optical centre, noting that the two optical density peaks occur $\sim 2$ arcmin apart, with elongation along the north-south axis as seen in the $\mathrm{X}$-ray and radio emission. Thus this new, unambiguous detection of a radio halo in Abell 0141 supports the previous findings in the literature of the association of radio haloes in merging clusters.

The radio halo is measured to have a flux density of $S_{168}=110 \pm 11 \mathrm{mJy}$ and an LAS of 5.51 arcmin, which translates to an LLS of $1.250 \mathrm{Mpc}$. This LLS puts the radio halo within the class of giant radio haloes $(\mathrm{GRH})$ defined to be greater than $1000 \mathrm{kpc}$ in extent. An upper limit to the $1.4 \mathrm{GHz}$ flux density is estimated to be $S_{1.4} \leq 13.7 \mathrm{mJy}$ which places an upper limit on the spectral index as $\alpha_{168}^{1.4} \leq-0.98 \pm 0.05$. A similar limit from the $147.5 \mathrm{MHz}$ TGSS survey would suggest a spectral index $\alpha_{147.5}^{168} \geq-5.6 \pm 0.3$, though this lower limit is not useful for classification purposes. 

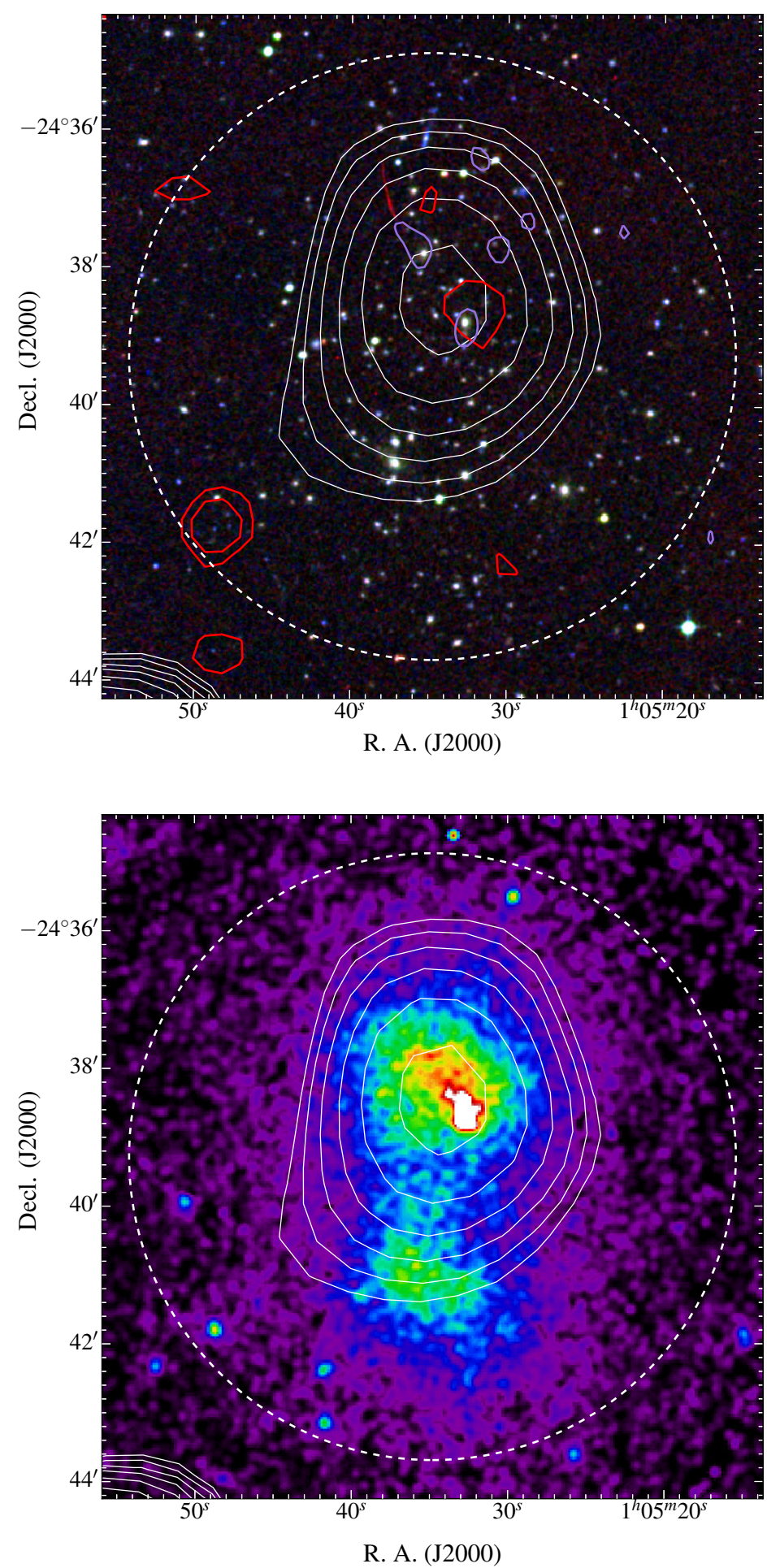

Figure ii.10: Radio halo at the centre of Abell 0141. Top: The background is an RGB image with contours overlaid as follows: EoR0, white, beginning at 10 mJy beam $^{-1}$ and increasing by a factor of $\sqrt{2}$; NVSS, red, beginning at 1.5 mJy beam ${ }^{-1}$ and increasing by a factor of 2 ; TGSS, medium purple, beginning at $13.8 \mathrm{mJy}$ beam $^{-1}$ also increasing by a factor of 2. Bottom: Exposure corrected, smoothed XMM-Newton data (Obs. ID 0693010501, PI Zhang) with EoR0 contours overlaid. In both panels the dashed circle is centred on the cluster with a $1 \mathrm{Mpc}$ radius. 
§ii.2.2.8. Abell 2496

Abell 2496 (MCXC J2251.0-1624; PSZ1 G047.75-60.16) has a redshift of $z=$ 0.1221 , an X-ray luminosity of $L_{500}=2.030655 \times 10^{37} \mathrm{~W}(\mathrm{MCXC})$, and mass $M_{\mathrm{YZ}, 500}=2.98_{-0.44}^{+0.41} \times 10^{14} \mathrm{M}_{\odot}(\mathrm{PSZ} 1)$. Fig. ii.11 shows the centre of the cluster with diffuse emission with an irregular morphology. Assuming that this emission is a single source and that any embedded galaxies do not show significant radio emission, the $168 \mathrm{MHz}$ flux density is measured to be $S_{168}=561 \pm 42 \mathrm{mJy}$, with an estimated LAS of $\sim 4.8$ arcmin translating to an LLS at the cluster's redshift of $\sim 650 \mathrm{kpc}$. The radio source is part of the NVSS catalogue as NVSS J225055-162721 from which we obtain the $1.4 \mathrm{GHz}$ flux density of $S_{1.4}=37.7 \pm 2.0$ mJy (Condon et al., 1998). We obtain flux densities from the TGSS and VLSSr catalogues for sources at the position of the halo of $S_{147.5}=659.4 \pm 67.0 \mathrm{mJy}$ (Intema et al., 2016) and $S_{74}=1.34 \pm 0.25 \mathrm{Jy}$ (Lane et al.). With these and our $168 \mathrm{MHz}$ measurement we obtain a spectral index across the frequency range $74-1400 \mathrm{MHz}$ of $\alpha=-1.26 \pm 0.02$. This is consistent with typical cluster halo spectral indices (Feretti et al., 2012), however it is clear the $168 \mathrm{MHz}$ flux density is being measured over a larger area, as well as including more sources, than the $147.5 \mathrm{MHz}$ TGSS and 1.4 GHz NVSS measurements. This is clearly seen in Fig. ii.11 where there is much more emission to the north of the source, which may be unassociated and is not incorporated in the TGSS and NVSS catalogue values. The emission, while seen in the TGSS and NVSS contours at $3 \sigma_{\mathrm{rms}}$, is not measured for the respective catalogues. Further, due to the higher resolution of the TGSS images, 147.5 MHz flux density measurements of extended emission may be under-estimated due to resolving out flux on larger spacial scales. This has less of an affect in the NVSS images. We note that the TGSS ADR1 has flux density discrepancies, where variations of more than ten per cent are seen within certain parts of the survey ${ }^{5}$. This does not affect all regions within the survey, and in the case of Abell 2496 the flux density in this region does not vary by more than five per cent from the equivalent $151 \mathrm{MHz}$ flux density obtained from the GLEAM survey, present in GLEAM extragalactic catalogue (GLEAM EGC; Hurley-Walker et al., 2017), so we consider the TGSS measurement as accurate on the spacial scales it samples. The bulk of the radio emission is offset from the $\mathrm{X}$-ray emission seen with XMM-Newton data in the bottom panel of Fig. ii.11 (Obs. ID 0765030801, PI Reiprich). The emission does extend towards the X-ray peak, however given the offset the emission is more likely that of a relic. The slight extension of the $168 \mathrm{MHz}$ emission along with the patchy $1.4 \mathrm{GHz}$ NVSS and $147.5 \mathrm{MHz}$ TGSS at the peak of the X-ray may suggest a particularly faint or small halo at the centre of the cluster, perhaps a mini-halo. 

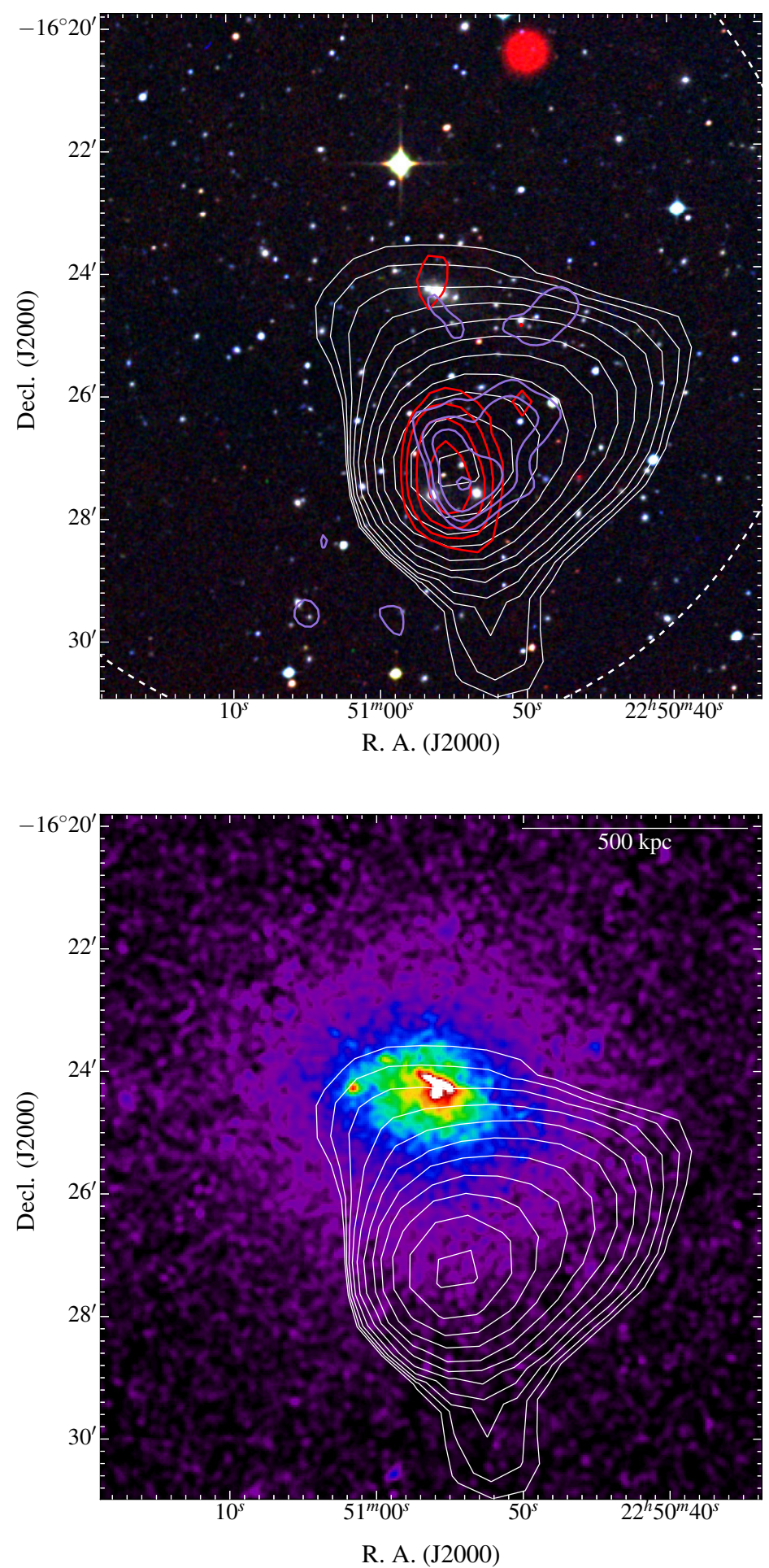

Figure ii.11: Diffuse emission within Abell 2496. Top: The background is an RGB image with contours overlaid as follows: EoR0, white, beginning at $15 \mathrm{mJy}_{\text {beam }}{ }^{-1}$ increasing by a factor of $\sqrt{2}$; NVSS, red, beginning at 1.5 mJy beam $^{-1}$ increasing by a factor of 2 . TGSS, medium purple, $12 \mathrm{mJy}$ beam $^{-1}$ also increasing by a factor of 2. The dashed, white circle is centred on the MCXC coordinates with radius of 1 Mpc. Bottom: Exposure corrected, smoothed XMM-Newton data overlaid with MWA contours as in the top panel. 


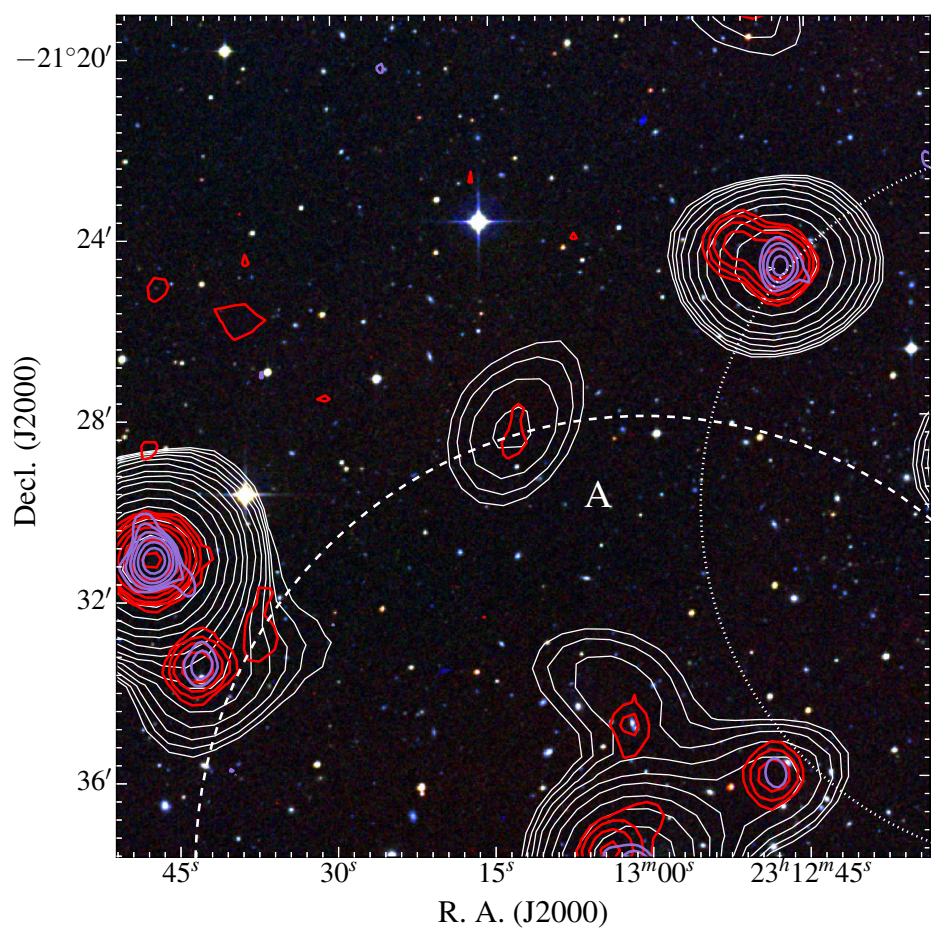

Figure ii.12: Diffuse emission, Obj. A, in Abell 2556. The background image is an RGB image with contours overlaid as follows: EoR0, white, beginning at $10 \mathrm{mJy}$ beam $^{-1}$ and increasing by a factor of $\sqrt{2}$; NVSS, red, beginning at 1.5 mJy beam ${ }^{-1}$ and increasing by a factor of 2 ; TGSS, medium purple, beginning at $13.4 \mathrm{mJy}_{\text {beam }}{ }^{-1}$ also increasing by a factor of 2 . The dashed circle is centred on Abell 2556 and the dotted circle on Abell 2554, each with radii of $1 \mathrm{Mpc}$.

\section{\&ii.2.2.9. Abell 2556 and Abell 2554}

Fig. ii.12 shows the two clusters Abell 2556 (MCXC J2313.0-2137) and Abell 2554 (MCXC J2312.3-2130; PSZ1 G041.51-66.77) which have centres within 13 arcmin of each other, but have redshifts of $z=0.0871$ and $z=0.1108$ (Caretta et al., 2002) respectively. Their respective masses are $M_{\mathrm{X}, 500}=2.4758 \times 10^{14} \mathrm{M}_{\odot}(\mathrm{MCXC})$ and $M_{\mathrm{YZ}, 500}=3.05_{-0.39}^{+0.37} \times 10^{14} \mathrm{M}_{\odot}(\mathrm{PSZ} 1)$. Abell 2556 has an X-ray luminosity of $L_{500}=1.509152 \times 10^{37} \mathrm{~W}(\mathrm{MCXC})$. To the north of Abell 2556, $1 \mathrm{Mpc}$ from its centre (east of Abell 2554, over $1 \mathrm{Mpc}$ ) an elongated diffuse source is seen, labelled "A" in Fig. ii.12, with flux densities $S_{168}=29.3 \pm 5.5 \mathrm{mJy}$ (this work) and $S_{1.4}=2.2 \pm 0.5 \mathrm{mJy}$ (Condon et al., 1998). From these we obtain a steep spectral index of $\alpha_{168}^{1.4}=-1.22 \pm 0.14$. The LAS of the source is 3.33 arcmin which suggests an LLS of $336 \mathrm{kpc}$ at $z=0.0871$ or $416 \mathrm{kpc}$ at $z=0.1108$. The size of the emission does not suggest a radio relic. With no visible optical galaxy at the centre of the emission, lack of significant elongation, size, and steep spectral index suggest a radio phoenix. However, we note that radio phoenices are more often found towards cluster centres but this would be consistent with the spectral index, where phoenices closer to the centre become much steeper.

\footnotetext{
${ }^{5}$ http://tgssadr.strw. leidenuniv.nl/doku.php?id=knownproblems
} 
§ii.2.2.10. Abell 2680

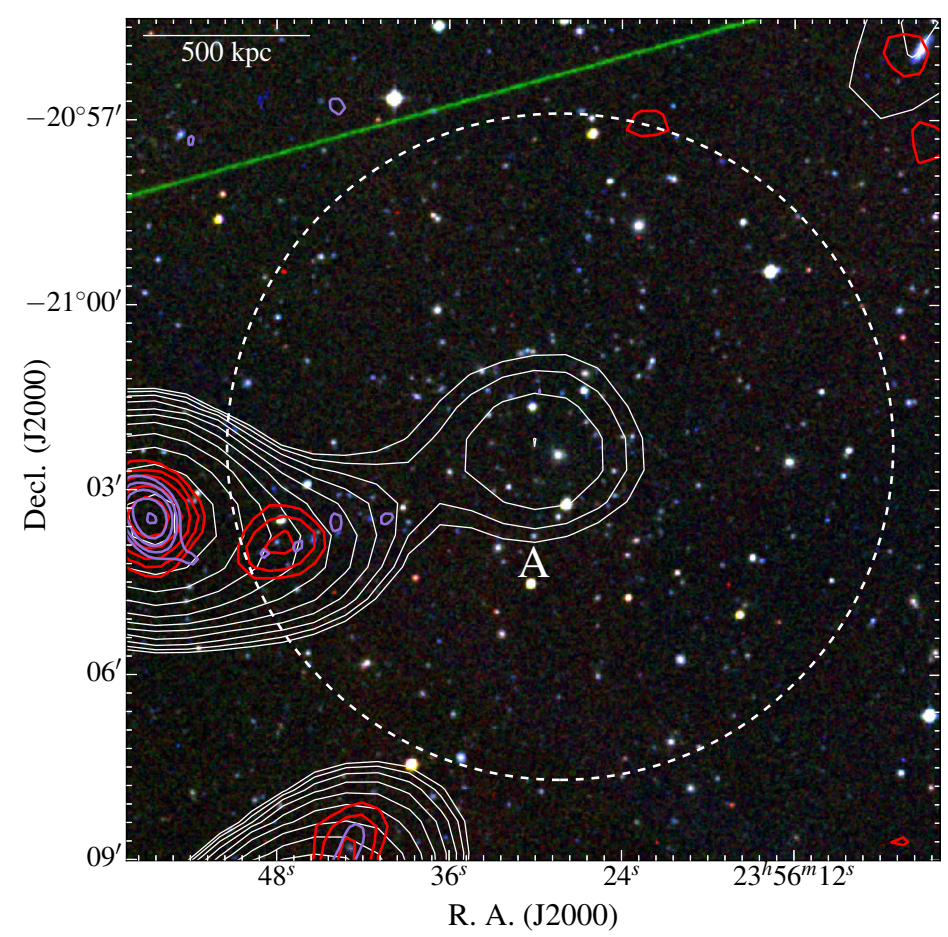

Figure ii.13: Abell 2680. The background image is an RGB image with contours overlaid as follows: EoR0, white, beginning at $7 \mathrm{mJy}_{\text {beam }}{ }^{-1}$ and increasing by a factor of $\sqrt{2}$; NVSS, red, beginning at $1.5 \mathrm{mJy}_{\text {beam }}{ }^{-1}$ and increasing by a factor of 2; TGSS, medium purple, beginning at $11.1 \mathrm{mJy}^{\text {beam }}{ }^{-1}$ also increasing by a factor of 2 . The dashed circle represents is at a $1 \mathrm{Mpc}$ radius about the cluster centre.

Abell 2680 has a photometric redshift of $z=0.1771$ (Wen and Han, 2013). Fig. ii.13 shows a patch of steep-spectrum emission at the centre of the cluster, where both the TGSS and NVSS surveys show no counterparts (Obj. A). The emission may be slightly elongated east-west, though this apparent elongation may just be the result of blending with the eastern sources. The steep-spectrum emission blends with the eastern NVSS sources: NVSS J235647-210352 and NVSS J235656-210326. The eastern-most source, NVSS J235656-210326, has a counterpart in the TGSS however the other source does not and so no spectral index can be calculated thus we do not predict a $168 \mathrm{MHz}$ flux density for the source. We make an approximate measurement of the flux density yielding $S_{168}=22.8 \pm 8.0$ mJy, where the uncertainty is given by Eq. ii.1 with an additional contribution to account for the slight blending to the east. We estimate a $1.4 \mathrm{GHz}$ upper limit of $1.8 \mathrm{mJy}$ to provide an upper limit to the spectral index between $168-1400 \mathrm{MHz}$, $\alpha_{168}^{1.4} \leq-1.2 \pm 0.2$. The LAS is estimated to be $\sim 3.2$ arcmin, which at $z=0.1771$ suggests a projected LLS of $600 \mathrm{kpc}$. The physical extent of the source and coincidence with the cluster centre core suggests a cluster halo. This particular case requires observations at different resolutions to determine if the source is actually extended. 


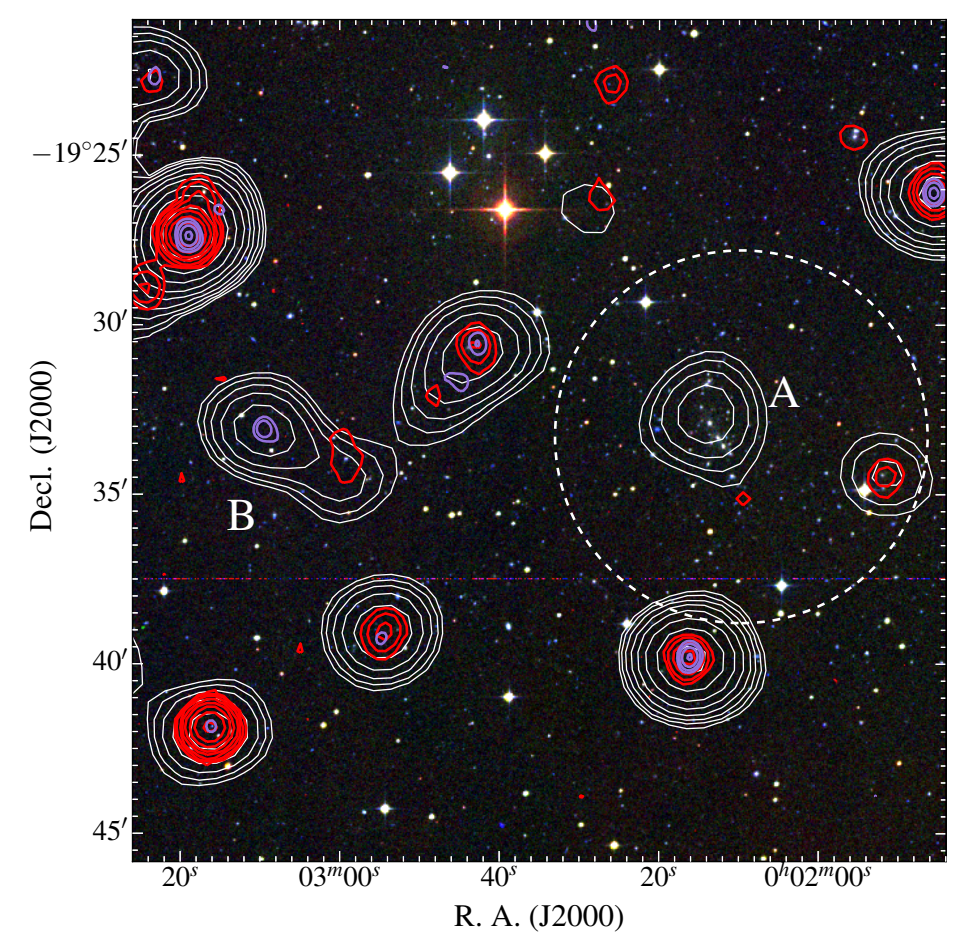

Figure ii.14: Candidate radio halo A and steep-spectrum source B within and nearby Abell 2693. The background is an RGB image with contours overlaid as follows: EoR0, white, beginning at $10 \mathrm{mJy} \mathrm{beam}^{-1}$ and increasing by a factor of $\sqrt{2}$; NVSS, red, beginning at $1.5 \mathrm{mJy}_{\text {beam }}^{-1}$ and increasing by a factor of 2 ; TGSS, medium purple, beginning at $12 \mathrm{mJy}$ beam $^{-1}$ also increasing by a factor of 2 . The dashed circle is centred on the cluster and has a $1 \mathrm{Mpc}$ radius.

Abell 2693 has a photometric redshift of $z=0.173453$ (Coziol et al., 2009) and hosts a candidate radio halo at its centre. The candidate halo, marked A in Fig. ii.14, has an LAS of 3.75 arcmin and an LLS at the cluster's redshift of 681 kpc. The location of the halo traces the optical galaxies. We measure the $168 \mathrm{MHz}$ flux density to be $S_{168}=49.6 \pm 6.0 \mathrm{mJy}$. From the lack of $1.4 \mathrm{GHz}$ emission in the NVSS, we estimate an upper limit to the $1.4 \mathrm{GHz}$ flux density to be $S_{1.4} \leq 7.7$ mJy which imposes an upper limit on the spectral index of $\alpha_{168}^{1.4} \leq-0.88 \pm 0.06$. This limit on the spectral index is in no way conclusive of the classification of radio halo. However, the location, and size suggest that it may be a halo, and we classify this emission as a candidate halo.

To the west of the cluster there is an elongated steep-spectrum source marked B in Fig. ii.14. If the entirety of Obj. B is a single object, then there is a spectral gradient across the source, with a steeper spectral index towards the northeast. It is unlikely this is a cluster relic associated with Abell 2693 as it sits at $\sim 2.5 \mathrm{Mpc}$ from the cluster centre and has the morphology of a head-tail (HT) radio galaxy (Miley et al., 1972). 
Table ii.3: $168 \mathrm{MHz}$ flux densities and spectral indices calculated for blended sources near the centre of Abell 2721.

\begin{tabular}{lcc}
\hline \hline Source & $\begin{array}{c}S_{168} \\
(\mathrm{mJy})\end{array}$ & $\alpha$ \\
\hline \multicolumn{3}{c}{ Prediction using spectral index } \\
\hline NVSS J000553-344434 & $183.5 \pm 6.2$ & $-0.88 \pm 0.04$ \\
NVSS J000614-344730 & $292 \pm 16$ & $-1.15 \pm 0.07$ \\
Diffuse emission & $34 \pm 33$ & $\leq-0.74 \pm 0.04$ \\
\hline \multicolumn{4}{c}{ Measured with AEGEAN } \\
\hline NVSS J000553-344434 & $234 \pm 23$ & - \\
NVSS J000614-344730 & $276 \pm 27$ & - \\
Diffuse emission & $54 \pm 14$ & $\leq-0.96 \pm 0.12$ \\
\hline
\end{tabular}

§ii.2.2.12. Abell 2721

Fig. ii.15 shows Abell 2721 (MCXC J0006.0-3443; PSZ1 G352.35-77.66) which has a redshift of $z=0.114412 \pm 0.000334$ (Zaritsky et al. 2006) with mass $M_{\mathrm{YZ}, 500}=$ $3.77_{-0.37}^{+0.35} \times 10^{14} M_{\odot}(\mathrm{PSZ} 1)$ and X-ray luminosity $L_{500}=1.809494 \times 10^{37} \mathrm{~W}(\mathrm{MCXC})$. Diffuse radio emission is seen at $168 \mathrm{MHz}$ offset to the east of the cluster centre (Obj. A in Fig. ii.15). No emission is detected at $1.4 \mathrm{GHz}$ in NVSS, $843 \mathrm{MHz}$ in SUMSS, or $147.5 \mathrm{MHz}$ in TGSS above their respective $\sim 3 \sigma_{\text {rms }}$ levels. The lack of emission seen in NVSS or SUMSS suggests a steep spectral index, however the lack of emission in TGSS is likely due to lack of sensitivity to extended, low surface brightness emission.

As part of the ATCA REXCESS Diffuse Emission Survey (ARDES), deep 1.4 and $2.1 \mathrm{GHz}$ imaging of the cluster was obtained with the ATCA, finding no evidence of diffuse emission (Shakouri et al., 2016). Given that Abell 2721 was one of the more disturbed clusters in the ARDES sample, the lack of radio halo in the ATCA imaging was previously noted and a upper limit to the $1.4 \mathrm{GHz}$ flux density is provided by Shakouri et al. (in prep.). This limit is $S_{1.4} \leq 7 \mathrm{mJy}$. We estimate the LLS of the diffuse emission to be $\sim 460 \mathrm{kpc}$. In an attempt to get a flux estimate for the diffuse emission at $168 \mathrm{MHz}$, we first calculate the spectral indices of the western and southern point sources, NVSS J000553-344434 and NVSS J000614344730 respectively, such that we might predict the flux density contribution from these sources to the region and subtract them. For NVSS J000553-344434 we obtain catalogue flux densities of $S_{1.4}=29.3 \pm 1.3$ mJy (Condon et al., 1998), $S_{843}=42.4 \pm 1.7 \mathrm{mJy}$ (Mauch et al.), and $S_{147.5}=218.5 \pm 22.4 \mathrm{mJy}$ (Intema et al., 2016). These yield a spectral index of $\alpha=-0.88 \pm 0.04$, giving a $168 \mathrm{MHz}$ flux density of $S_{168}=183.5 \pm 6.2$ The uncertainty in the predicted flux density is obtained through standard unbiased prediction uncertainty (see Wolberg, 2006, pp. 54-57). Similarly, for NVSS J000614-344730 we obtain catalogue flux densities of $S_{1.4}=24.5 \pm 0.9 \mathrm{mJy}$ (Condon et al., 1998), $S_{843}=49.7 \pm 2.1 \mathrm{mJy}$ (Mauch et al., 2003), and $S_{147.5}=305.4 \pm 30.9$ mJy (Intema et al., 2016), yielding the spectral 

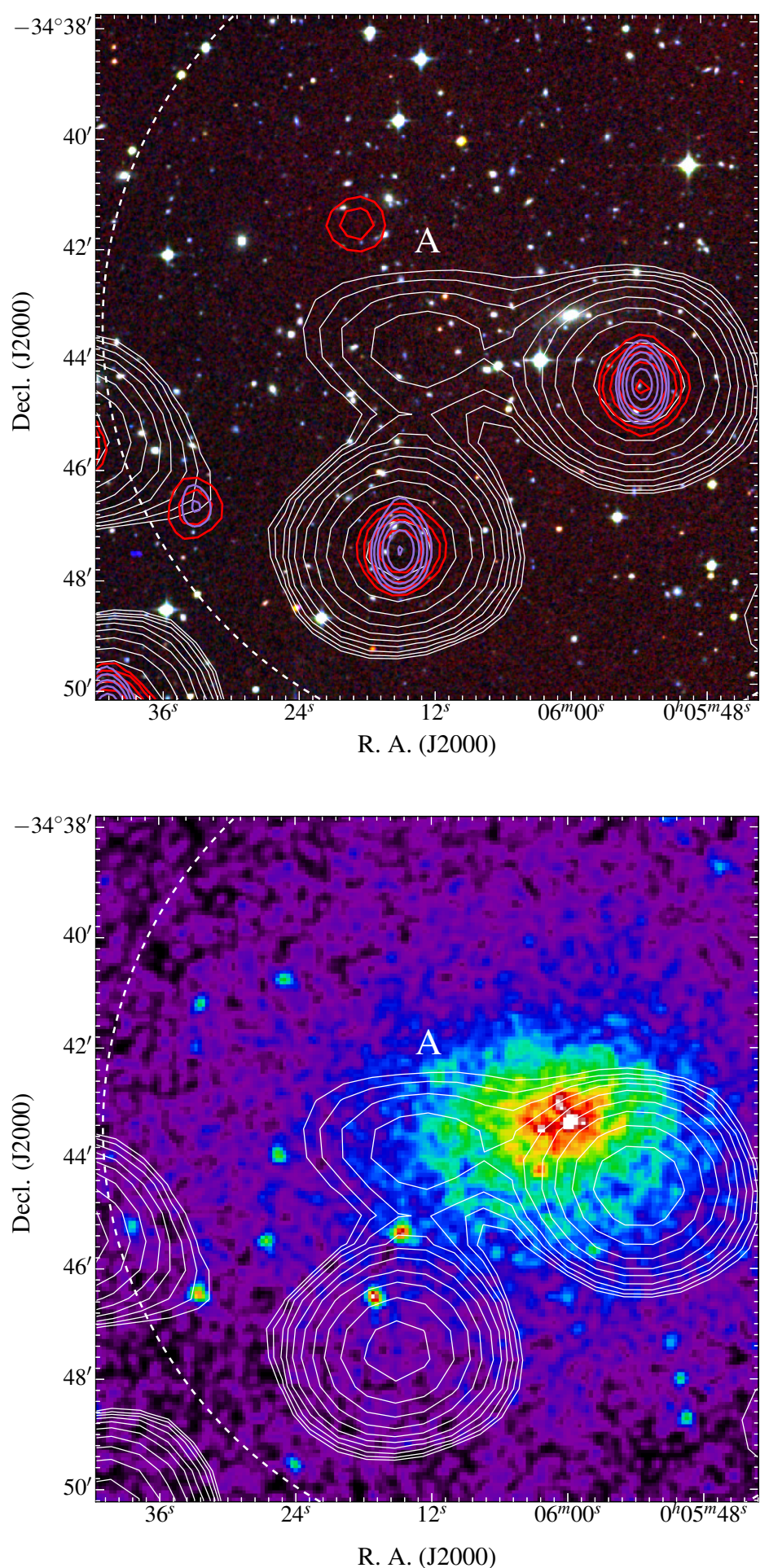

Figure ii.15: Diffuse emission within Abell 2721. Top: The background is an RGB image formed using the DSS2 red, IR, and blue bands, with contours overlaid as follows: EoR0, white, beginning at $10 \mathrm{mJy}$ beam $^{-1}$ increasing by a factor of $\sqrt{2}$; NVSS, red, beginning at $1.5 \mathrm{mJy}_{\text {beam }}{ }^{-1}$ increasing by a factor of 2; TGSS, medium purple, beginning at $8.1 \mathrm{mJy}$ beam $^{-1}$ also increasing by a factor of 2 . Bottom: X-ray image from the REXCESS survey with EoR0 contours overlaid as in the top panel along with X-ray contours increasing with a factor of 2 in black. The dashed circle is centred on the cluster with a radius of $1 \mathrm{Mpc}$. 
index $\alpha=-1.15 \pm 0.07$ giving $S_{168}=292 \pm 16$. The entire structure (diffuse emission, NVSS J000553-344434 and NVSS J000614-344730) is measured to have a flux density of $S_{168}^{\text {tot }}=509 \pm 28$ mJy. Subtracting the point sources gives $S_{168}=$ $34 \pm 33 \mathrm{mJy}$. The large uncertainty in this estimation is the result of combining predicted $168 \mathrm{MHz}$ measurements. This estimate along with the ATCA $1.4 \mathrm{GHz}$ limit provides a spectral index limit of $\alpha_{168}^{1.4} \leq-0.74 \pm 0.04$. We compare these results to those obtained by AEGEAN, which is able to separate out the three components of the structure. This approach is successful, and yields a flux density of $S_{168}=54 \pm 14$ for the diffuse emission, which is consistent with the method of summing the flux density contributions of each pixel within a box roughly surrounding the diffuse emission. The likely cause of the discrepancy are the SED's between 147.5 and $1400 \mathrm{MHz}$, and using those to predict a $168 \mathrm{MHz}$ flux density. We use AEGEAN again to measure the flux densities of NVSS J000553-344434 and NVSS J000614-344730, arriving at $S_{168}=236 \pm 23 \mathrm{mJy}$ and $S_{168}=276 \pm 27 \mathrm{mJy}$ respectively. The total flux of the structure of three sources is then $S_{168}^{\text {tot }}=565 \pm 38$ mJy, which disagrees with the previously measured value using the in-house code, though they agree within their uncertainties. Table ii. 3 summarises the $168 \mathrm{MHz}$ flux densities and spectral indices of the blended sources. The extra flux density likely originates from setting a floodclip value in AEGEAN of $2 \sigma_{\mathrm{rms}}$, which is less than the hard $3 \sigma_{\text {rms }}$ cutoff used with the in-house code. The difference in total flux of the structure is not large enough to be a significant cause of the discrepancy between individual source calculations. Predicting flux densities by way of spectral modelling can fail dramatically if the spectrum is not sampled finely enough to catch the true nature of the SED. We assume that both NVSS sources can be fit with simple power laws. This may not be the case, and without finer sampling in the vicinity of $168 \mathrm{MHz}$ it is difficult to tell whether the power law fits are accurate. Despite this, the AEGEAN result and predicted value of the diffuse emission are consistent to within their uncertainties, though they are large.

The bottom panel of Fig. ii.15 shows the REXCESS X-ray data overlaid with MWA contours. We see that the X-ray emission sits at the centre of the cluster, whereas the diffuse emission seen only at $168 \mathrm{MHz}$ is offset towards the east. While there is the possibility that this is a cluster halo, elongated along the E-W direction and partially obscured by the emission from NVSS J000553-344434, it is more likely that this is a radio relic akin to the relics seen in Abell 0013 or Abell 0085. This candidate relic may be projected onto the centre of the cluster, and its reasonably flat spectral index compared to other relics would be consistent with a peripheral relic. Hence because of its offset and size, and in the absence of any available polarimetry, we classify this new diffuse emission as a candidate radio relic.

\section{§ii.2.2.13. Abell 2744}

Abell 2744 (MCXC J0014.3-3022; PSZ1 G009.02-81.22) is a distant massive X-ray luminous cluster $\left(M_{\mathrm{YZ}, 500}=7.3614 \times 10^{14} \mathrm{M}_{\odot}(\mathrm{PSZ1}), z=0.3066\right.$, and $L_{500}=$ 


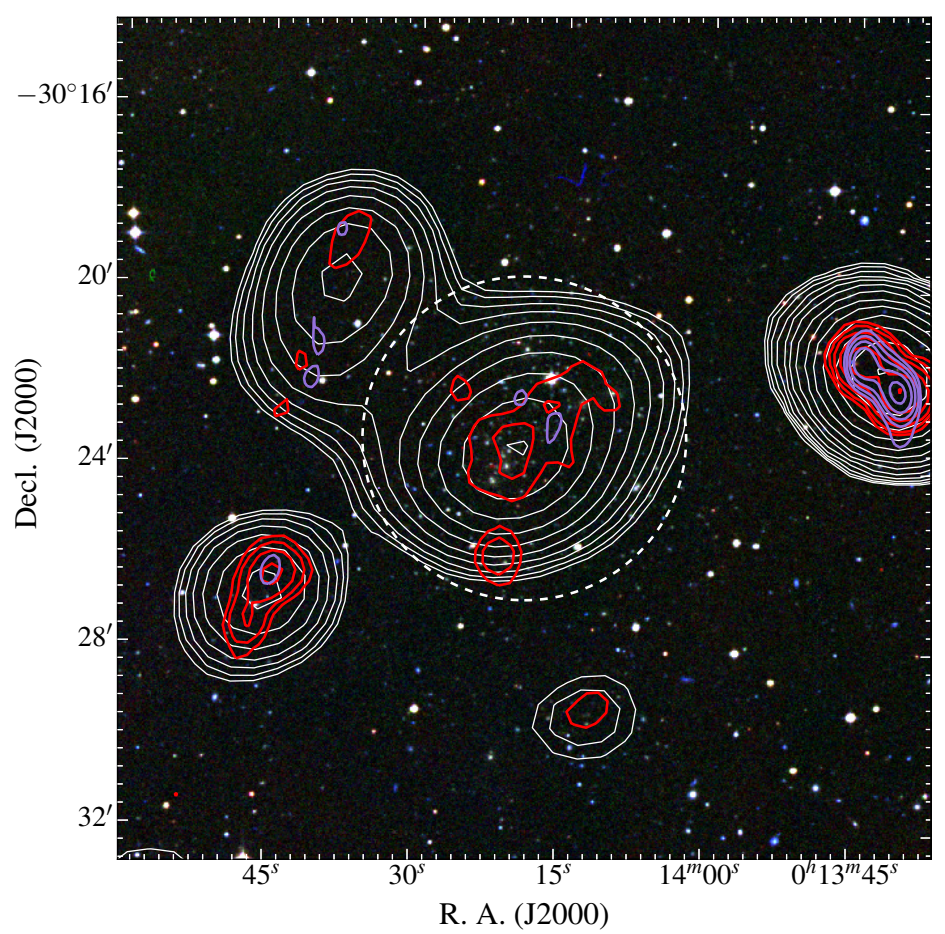

Figure ii.16: Abell 2744 with giant radio halo and relic. The background is an RGB image with contours overlaid as follows: EoR0, white, beginning at $10 \mathrm{mJy}^{\text {beam }}{ }^{-1}$ and increasing by a factor of $\sqrt{2}$; NVSS, red, beginning at $1.5 \mathrm{mJy}^{\text {beam }}{ }^{-1}$ and increasing by a factor of 2 ; TGSS, medium purple, beginning at $12.9 \mathrm{mJy}^{-1}$ beam $^{-1}$ also increasing by a factor of 2 . The dashed circle is centred on the cluster with a radius of $1 \mathrm{Mpc}$.

$\left.11.818114 \times 10^{37} \mathrm{~W}(\mathrm{MCXC})\right)$ and is a Hubble Frontier Fields cluster (Lotz et al., 2014) due to its mass and redshift, showing gravitational lensing of the highredshift background galaxies (see Castellano et al., 2016, for a catalogue of lensed galaxies with redshift and magnification data). Fig. ii.16 shows Abell 2744 with both a centrally located giant radio halo $(\mathrm{GRH}$, defined to have an LLS > $1 \mathrm{Mpc}$ ) and a mega-parsec scale radio relic on its northeast periphery (Govoni et al., 2001). Both of these objects are seen in the EoR0 field at $168 \mathrm{MHz}$, as is expected with such steep-spectrum sources, blending together along that northeast edge of the cluster. As seen in Fig. ii.16, the giant radio halo fills the the entire cluster out to $1 \mathrm{Mpc}$ having an approximate LLS of $2.14 \mathrm{Mpc}$ and the relic with an LLS on the order $1.49 \mathrm{Mpc}$.

We use AEGEAN to measure the integrated flux densities of the halo and relic. AEGEAN detects the two as a single source and fits two components - one for each the halo and relic - which give integrated fluxes of $S_{168}^{\text {halo }}=550 \pm 51 \mathrm{mJy}$ and $S_{168}^{\text {relic }}=237 \pm 24 \mathrm{mJy}$. Venturi et al. (2013) obtain $325 \mathrm{MHz}$ measurements using the GMRT, and use those along with re-reduced $1.4 \mathrm{GHz}$ data from the VLA to estimate spectral indices of $-1.19_{-0.11}^{+0.08}$ and $-1.24 \pm 0.10$ for the halo and relic respectively. We use the $168 \mathrm{MHz}$ flux density measurements along with those present in Venturi et al. to obtain spectral indices of $\alpha_{\text {halo }}=-1.11 \pm 0.04$ and 
$\alpha_{\text {relic }}=-1.19 \pm 0.05$ for the halo and relic. There is little deviation and spectral indices calculated here agree to within the associated uncertainties, however the measurements by Venturi et al. (2013) exclude the bridge emission as well as at least one embedded point source, which are not excluded in these measurements. 


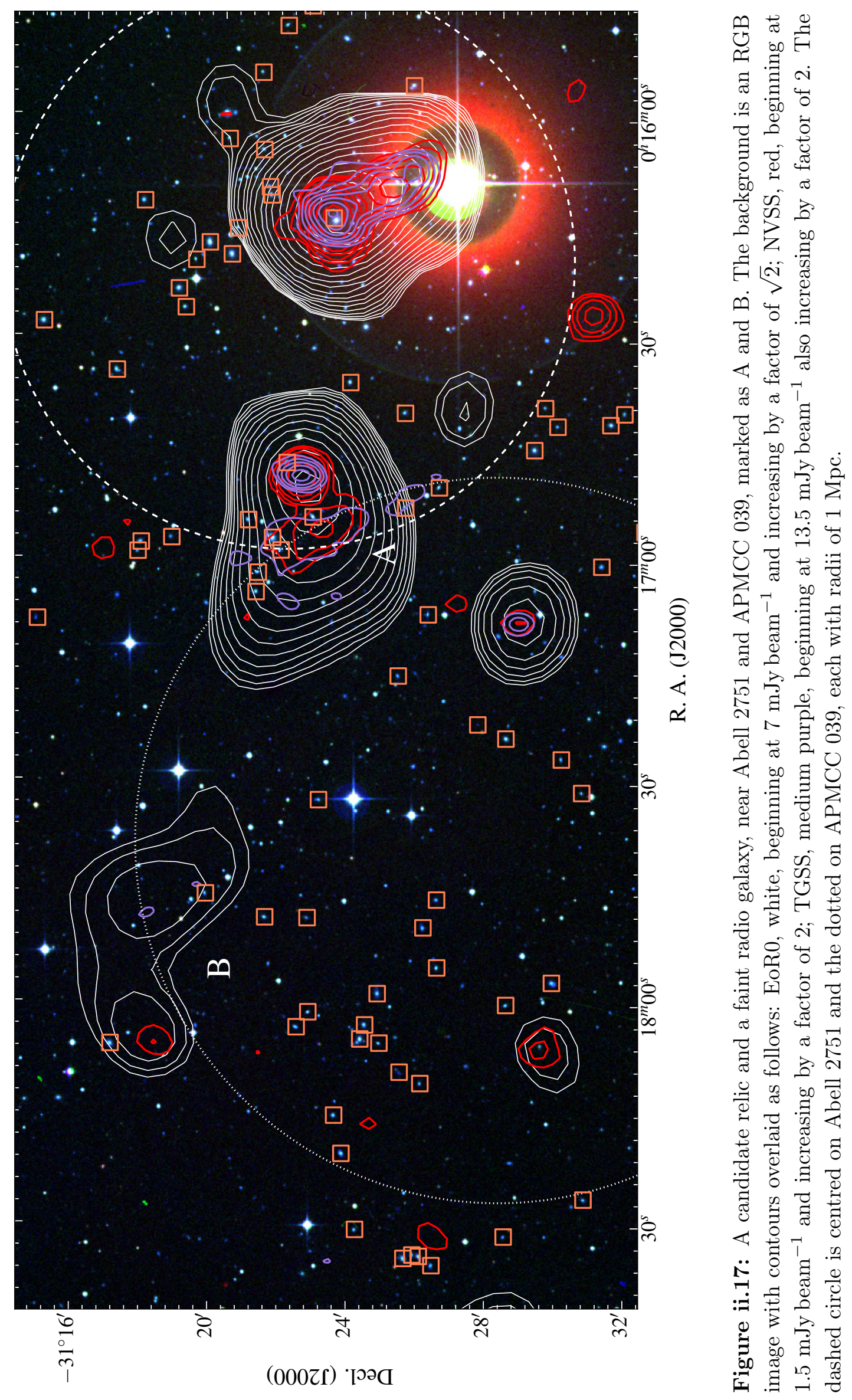




\section{§ii.2.2.14. Abell 2751 and APMCC 039}

Abell 2751 (MCXC J0016.3-3121, $L_{500}=0.495174 \times 10^{37} \mathrm{~W}$ from MCXC) and APMCC 039 have an angular separation of 17.7 arcmin and redshifts of $z=$ 0.107 (Struble and Rood, 1999) and $z=0.082$ (Dalton et al., 1997) respectively. However we note that the redshift for APMCC 039 is photometric, and so we compare the redshifts of the surrounding galaxies to check for the possibility that the two clusters may be interacting. Fig. ii.17 shows the two clusters, with the dashed and dotted circles indicating $1 \mathrm{Mpc}$ radii about the cluster centres at their reported redshifts. The small orange squares indicate galaxies with redshifts in the range $0.1-0.114$, which is $c z \approx 2000 \mathrm{~km} \mathrm{~s}^{-1}$ around the redshift of Abell 2751 . There are no galaxies in the vicinity at the reported redshift of APMCC 039. It is clear from Fig. ii.17 that the two clusters host galaxies of similar redshifts and due to their angular separation are likely interacting.

We detect a new candidate relic to the east of Abell 2751 (Obj. A in Fig. ii.17), blending with the point source NVSS J001648-312223. As the relic sits $\sim 1 \mathrm{Mpc}$ from both Abell 2751 and APMCC039, it may be associated with either cluster. In the case where the clusters are interacting, the relic sits at the intersection point between them. The relic itself is part of the NVSS catalogue as NVSS J001655-312258. The $168 \mathrm{MHz}$ emission appears to simply be an extended radio tail extending from NVSS J001648-312223, however the 147.5 MHz TGSS emission is resolved enough to show that the relic emission is not associated with the point source. Fig. ii.17 shows the emission around these clusters with an RGB image which does not show an obvious optical ID associated with the relic emission. The combined total flux density of the point source and relic is measured to be $S_{168}^{\text {tot }}=$ 843.1 $\pm 60.7 \mathrm{mJy}$. We obtain the following flux densities for NVSS J001648-312223 from catalogues: $S_{1.4}=55.0 \pm 2.1 \mathrm{mJy}$ (Condon et al., 1998), $S_{843}=91.4 \pm 3.2$ mJy (Murphy et al., 2007), $S_{147.5}=365.3 \pm 37.3 \mathrm{mJy}$ (Intema et al., 2016), and $S_{74}=1360 \pm 270 \mathrm{mJy}$ (Lane et al., 2014). Due to uncertainty in the TGSS ADR1 flux density scale in certain regions of the sky, we do not use the $147.5 \mathrm{MHz}$ flux density for sources in this region. We assume a standard power law and fit these measurements to obtain a spectral index $\alpha=-1.07 \pm 0.02$ which we use to predict the $168 \mathrm{MHz}$ flux density as $S_{168}=518.2 \pm 9.4 \mathrm{mJy}$. The flux density of the relic is then estimated to be $S_{168}^{\text {relic }}=324.9 \pm 61.4 \mathrm{mJy}$.

To the north of APMCC 039 is the radio source NVSS J001804-311824 (Obj. B in Fig. ii.17), which at $1.4 \mathrm{GHz}$ in the NVSS appears as a discrete point source with a flux density of $S_{1.4}=4.0 \pm 0.6 \mathrm{mJy}$ (Condon et al., 1998). The EoR0 field however shows emission extended far beyond this source, appearing as a faint tail to this possible radio galaxy. The entire emission is measured to have a $168 \mathrm{MHz}$ flux density of $S_{168}=60.3 \pm 7.7 \mathrm{mJy}$ and an LAS of 8.816 arcmin which at the reported redshift of APMCC 039 translates to an LLS of $840.8 \mathrm{kpc}$, or $1066 \mathrm{kpc}$ at the redshift of Abell 2751. If we take the catalogue $1.4 \mathrm{GHz}$ flux density to be a lower limit to the flux, we can estimate an upper limit to the flux density across the entire source to be $S_{1.4} \leq 24.0 \pm 0.6 \mathrm{mJy}$. We determine limits to the spectral 


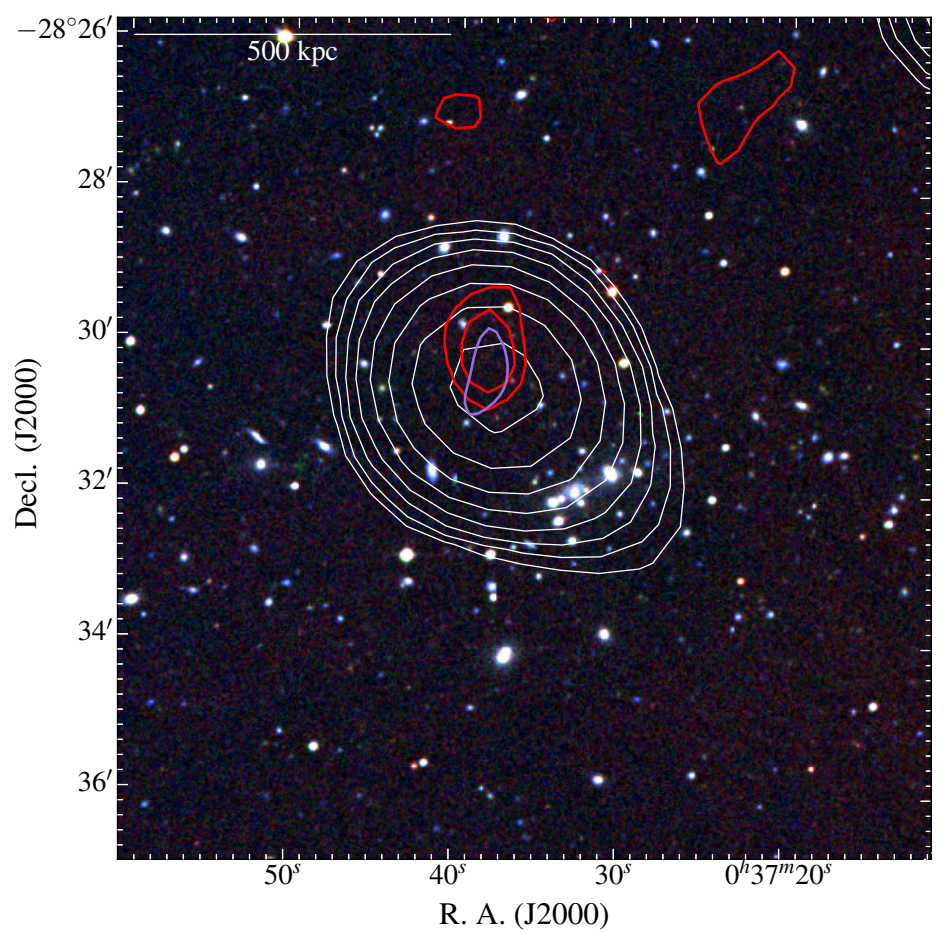

Figure ii.18: Candidate radio relic within Abell 2798. The background is an RGB image with contours overlaid as follows: EoR0, white, beginning at $7 \mathrm{mJy}^{\text {beam }}{ }^{-1}$ increasing by a factor of $\sqrt{2}$; NVSS, red, beginning at $1.5 \mathrm{mJy}_{\text {beam }}{ }^{-1}$ increasing by a factor of 2; TGSS, medium purple, beginning at $13.8 \mathrm{mJy}_{\text {beam }}^{-1}$ also increasing by a factor of 2 . The dashed circle is centred on the cluster with a radius of $1 \mathrm{Mpc}$.

index to be $-1.28( \pm 0.09) \leq \alpha_{168}^{1.4} \leq-0.43( \pm 0.06)$. This range covers normal radio galaxies, flat-spectrum sources, and most steep-spectrum sources so is not useful for classification. The emission lacks a clear optical ID, which may suggest that it is in fact a radio relic associated with APMCC 039. In the case of interacting clusters this is a distinct possibility. However with the morphology and range of spectral indices, we suggest that the emission is from likely a radio galaxy with a potential core described by the source NVSS J001804-311824.

\section{§ii.2.2.15. Abell 2798}

Abell 2798 (MCXC J0037.4-2831) has a redshift of $z=0.105$ (Struble and Rood, 1999) with mass $M_{\mathrm{X}, 500}=1.3146 \times 10^{14} \mathrm{M}_{\odot}$ and X-ray luminosity $L_{500}=0.545534 \times$ $10^{37} \mathrm{~W}$ (MCXC). Fig. ii.18 shows the cluster centre hosting a steep-spectrum radio source, slightly offset from centre. This radio source has a flux density of $S_{168}=110.3 \pm 9.4 \mathrm{mJy}$ and LAS of 4.84 arcmin, which at the cluster's redshift translates to an LLS of $575 \mathrm{kpc}$. Both the TGSS and NVSS surveys show extended emission offset slightly from the centroid of the $168 \mathrm{MHz}$ emission. However there are no optical IDs for this emission. The NVSS source is NVSS J003738-283008 and has a flux density of $S_{1.4}=9.0 \pm 1.3 \mathrm{mJy}$ (Condon et al., 1998). This yields a spectral index for the source of $\alpha_{168}^{1.4}=-1.18 \pm 0.08$. The RASS broad band (0.1- 


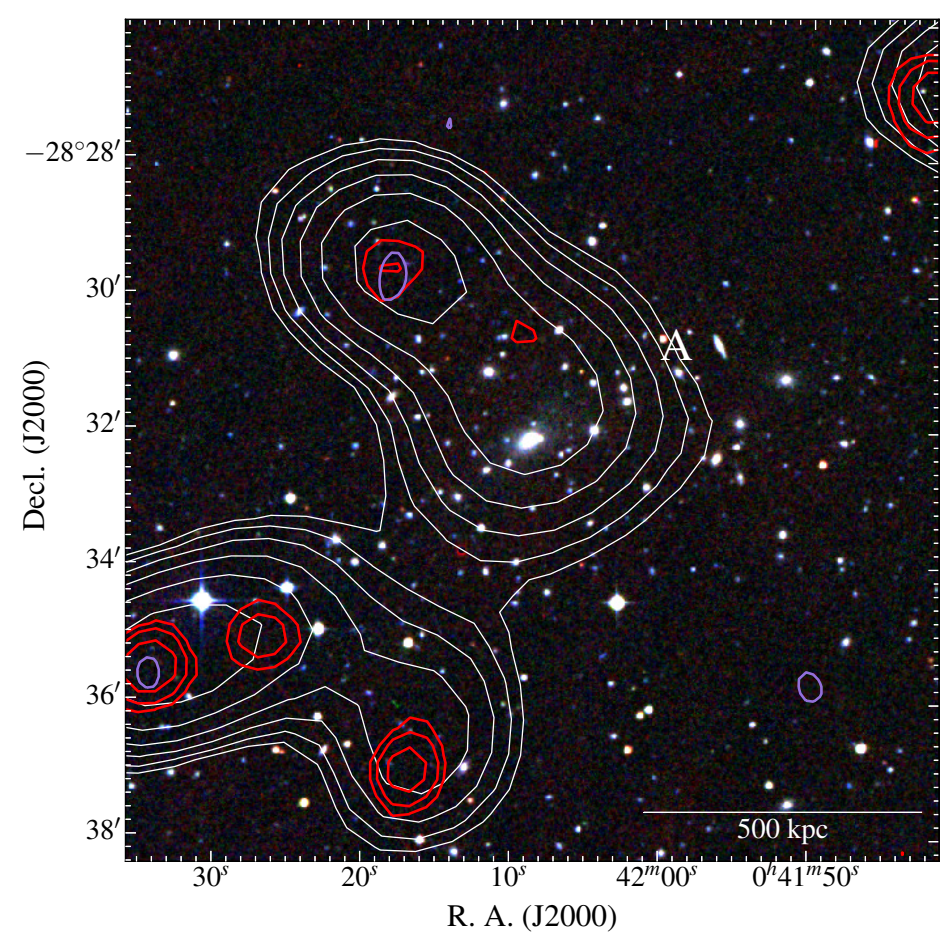

Figure ii.19: Radio halo within Abell 2811. The background is an RGB image with contours overlaid as follows: EoR0, white, beginning at $7 \mathrm{mJy}_{\text {beam }}{ }^{-1}$ and increasing by a factor of $\sqrt{2}$; NVSS, red, beginning at $1.5 \mathrm{mJy}^{-1}$ beam $^{-1}$ and increasing by a factor of 2; TGSS, medium purple, beginning at $12.6 \mathrm{mJy}_{\text {beam }}{ }^{-1}$ also increasing by a factor of 2 . The linear scale is at the redshift of the cluster.

$2.4 \mathrm{keV}$ ) count image shows no significant X-ray emission within the cluster which is consistent with the low cluster mass. We classify this emission similarly to that in Abell 0013: a candidate radio relic either near the cluster centre or projected onto it. Further high-resolution imaging will be necessary to fully determine the nature of this emission.

\section{§ii.2.2.16. Abell 2811}

The cluster Abell 2811 (MCXC J0042.1-2832; PSZ1 G357.94-87.52) has a redshift of $z=0.107908 \pm 0.000500$ (Zaritsky et al., 2006), an X-ray luminosity $L_{500}=$ $2.734146 \times 10^{37} \mathrm{~W}(\mathrm{MCXC})$, and mass $M_{\mathrm{YZ}, 500}=3.67_{-0.37}^{+0.35} \times 10^{14} \mathrm{M}_{\odot}(\mathrm{PSZ} 1)$. Fig. ii.19 shows the cluster and RGB image overlaid with MWA, NVSS, and TGSS contours. At the centre of the cluster we make a new detection of a faint radio halo (Obj. A in Fig. ii.19). The cluster has been studied previously within the context of galactic haloes and the enrichment of the ICM (e.g. Sivanandam et al., 2009) with particular focus on the BCG, 2MASX J00420892-2832087. Further, as part of the XMM-Newton survey of the soft X-ray background Henley and Shelton (2013) consider this emission a galactic halo. However, Sivanandam et al. note that the surrounding X-ray emission is offset from the BCG by $27 \operatorname{arcsec}(\sim 55 \mathrm{kpc})$, which suggests that the clusters is in a dynamic, merging state and that the low-frequency 


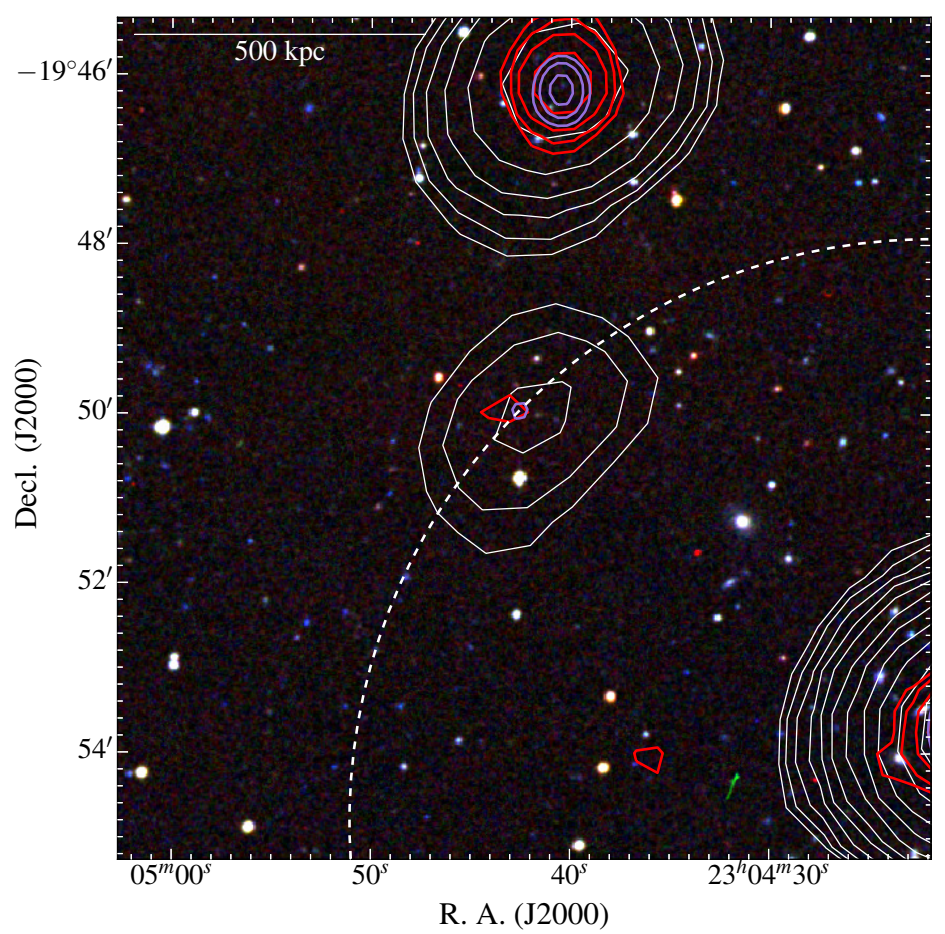

Figure ii.20: Abell 3964. The background is an RGB image with overlaid contours overlaid as follows: EoR0, white, beginning at $10 \mathrm{mJy} \mathrm{beam}^{-1}$ and increasing by a factor of $\sqrt{2}$; NVSS, red, beginning at $1.4 \mathrm{mJy}_{\text {beam }}{ }^{-1}$ and increasing by a factor of 2; TGSS, medium purple, beginning at $10.8 \mathrm{mJy}_{\text {beam }}{ }^{-1}$ also increasing by a factor of 2. The dashed circle is centred on Abell 3964 and has a radius of $1 \mathrm{Mpc}$.

radio emission seen in Fig. ii.19 is a cluster halo and not emission associated with the BCG. This radio halo is on the order of 4.13 armin, which translates to 502 kpc at the cluster's redshift, however the surrounding sources make it difficult to properly ascertain its true size. We measure a flux density of the halo region with AEGEAN, which fits a Gaussian component to the halo, separating it out from the nearby sources it blends with, obtaining $S_{168}=80.7 \pm 16.5 \mathrm{mJy}$. Considering the lack of detection in the NVSS image, we estimate an upper limit to the 1.4 $\mathrm{GHz}$ flux density of $3.1 \mathrm{mJy}$ which yields an upper limit to the spectral index of $\alpha_{168}^{1.4} \leq-1.5 \pm 0.1$. This would place the emission within the realm of the ultra-steep spectrum haloes.

\section{§ii.2.2.17. Abell 3964}

The cluster Abell 3964 has a faint, possibly steep-spectrum source on its NorthEastern periphery, about $1 \mathrm{Mpc}$ from the cluster centre. The cluster has a redshift of $z=0.1325$ (Caretta et al., 2002), and with an LAS of 2.84 arcmin this implies the emission has a projected LLS of $413 \mathrm{kpc}$. The emission has a flux density of $S_{168}=16.6 \pm 4.1$. Despite how faint the emission is at $168 \mathrm{MHz}$, at the $3 \sigma_{\mathrm{rms}}$ level both the NVSS and TGSS surveys show emission as well. There is a faint source visible in the DSS2 red band image at the centre of the image which is likely 


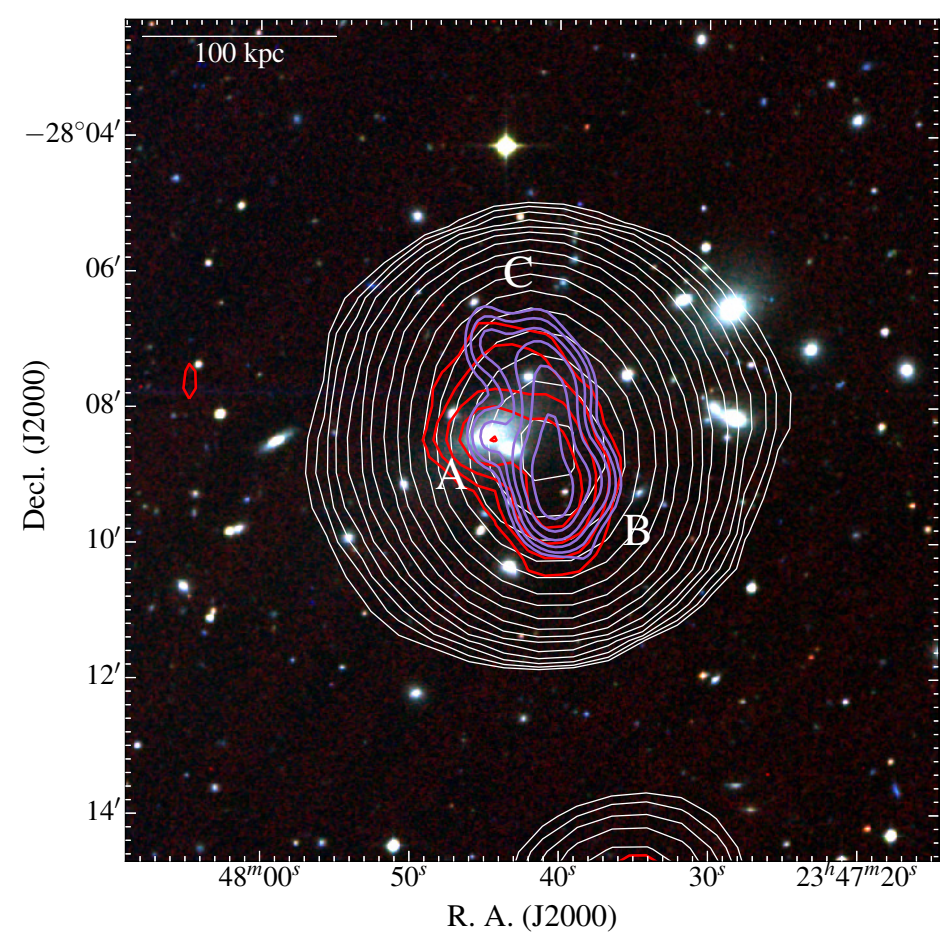

Figure ii.21: The centre of Abell 4038. The background is an RGB image with contours overlaid as follows: EoR0, white, beginning at $20 \mathrm{mJy}^{\text {beam }}{ }^{-1}$ and increasing with a factor of $\sqrt{2}$; NVSS, red, beginning at $1.5 \mathrm{mJy} \mathrm{beam}^{-1}$ and increasing with a factor of 2 ; TGSS, medium purple, beginning at $20 \mathrm{mJy}^{\text {beam }}{ }^{-1}$ also increasing with a factor of 2 . Marked objects are described in the text.

the host galaxy. There is no source in the NASA/IPAC Extragalactic Database (NED) at this position, however upon searching the VizieR service we find the source belongs to the USNO-B1.0 catalogue as USNO-B1.0 0701-0830366. We conclude that this is simply a faint, high-redshift radio galaxy coincidentally on the periphery of Abell 3964. This is left in as an example of such objects that were eventually rejected as, in particular, relic candidates.

\section{Sii.2.2.18. Abell 4038}

Abell 4038 (MCXC J2347.7-2808) is a nearby cluster with a redshift of $z=$ $0.028190 \pm 0.000550$ (Sanders et al., 2011), mass $M_{\mathrm{X}, 500}=2.0383 \times 10^{14} \mathrm{M}_{\odot}$ $(\mathrm{MCXC})$, and X-ray luminosity $L_{500}=1.029501 \times 10^{37} \mathrm{~W}(\mathrm{MCXC})$. A steepspectrum source (Obj. B and $\mathrm{C}$ in Fig. ii.21) is reported by Slee and Reynolds (1984) near the cluster centre without an optically visible host galaxy. Slee and Roy (1998) and Slee et al. (2001) then provide follow-up analyses of this steep-spectrum source and consider it a radio relic (defined as a radio phoenix here). As with Abell 0085 the $168 \mathrm{MHz}$ emission extends beyond the emission seen with the NVSS and TGSS. The emission of the phoenix blends with the radio emission from IC 5358 and 2MASX J23474209-2807335 (Obj. A in Fig. ii.21). Kale and Dwarakanath (2012) present a multi-frequency study of the phoenix with the GMRT, combining 
literature data with their 150,240,606, and $1288 \mathrm{MHz}$ data to subtract the interloping sources. We use results from their observations, as well as additional data from the NVSS at $1.4 \mathrm{GHz}$ (Condon et al., 1998) and at $29.9 \mathrm{MHz}$ from Slee et al. (2001; but see also Finlay and Jones 1973), to estimate the $168 \mathrm{MHz}$ contributions from IC 5358 and 2MASX J23474209-2807335 as 80.6 \pm 1.0 and $12.4 \pm 0.4 \mathrm{mJy}$, respectively. We measure the combined total flux to be $4.875 \pm 0.249 \mathrm{Jy}$ and arrive at $S_{168}=4.782 \pm 0.249$ Jy for the phoenix.

\section{§ii.2.2.19. Abell S0084}

Abell S0084 (MCXC J0049.4-2931) is a cluster added to the Abell catalogue as part of the supplementary catalogue. The cluster has a redshift of $z=0.108041 \pm$ 0.000400 (Zaritsky et al., 2006) and X-ray luminosity $L_{500}=1.438047 \times 10^{37} \mathrm{~W}$ (MCXC). We detect diffuse radio emission at the cluster centre (Fig. ii.22). The cluster was part of the ARDES sample of Shakouri et al. (2016) though no diffuse emission was detected at the centre of the cluster. From ATCA imaging at $1.4 \mathrm{GHz}$ Shakouri et al. obtain an rms noise of $313 \mu \mathrm{Jy}_{\text {beam }}{ }^{-1}$ from which we estimate an upper limit to the $1.4 \mathrm{GHz}$ flux density to be $S_{1.4} \leq 2.2 \mathrm{mJy}$ (Shakouri et al. in prep.). The centroid of the emission is offset from the cluster centre by 63 arcsec and has an LAS of 4.19 arcmin, which at the cluster's redshift is an LLS of $511 \mathrm{kpc}$. The flux density is measured to be $S_{168}=32.3 \pm 4.5 \mathrm{mJy}$. The $147.5 \mathrm{MHz}$ emission is not suggestive of a point source and the lack of detection by Shakouri et al. (2016) suggests a steep spectral index. With the $1.4 \mathrm{GHz}$ upper limit, we estimate an upper limit on the spectral index, $\alpha_{168}^{1400} \leq-1.3 \pm 0.1$. The right panel of Fig. ii.22 shows the REXCESS X-ray data with MWA contours overlaid. There is no cavity present in the X-ray data to suggest that the emission could be the lobes of an AGN and thus likely associated with the cluster itself. Further, Abell S0084 is not a cool core cluster (Pratt et al., 2009) and so we do not suspect this emission is a mini-halo. However the X-ray plasma appears undisturbed and the radio emission is offset from the X-ray peak by $\sim 100 \mathrm{kpc}$ so we only tentatively classify this emission as that of a candidate cluster halo, though note that the emission may be representative of an old or dead radio galaxy near the centre.

\section{\$ii.2.2.20. Abell S1063}

Abell S1063 (MCXC J2248.7-4431; PSZ1 G349.46-59.90) is a Hubble Frontier Fields cluster and features heavy gravitational lensing of the distant background optical galaxies (see e.g. Diego et al., 2016). The cluster has a redshift of $z=$ 0.3475 (Böhringer et al., 2004) with mass $M_{\mathrm{YZ}, 500}=11.41_{-0.44}^{+0.43} \times 10^{14} \mathrm{M}_{\odot}(\mathrm{PSZ} 1)$ and X-ray luminosity $L_{500}=27.166569 \times 10^{37} \mathrm{~W}(\mathrm{MCXC})$. The cluster is near the southern edge of the EoR0 field and so is more affected by noise. Despite this, above a $50 \mathrm{mJy}^{\text {beam }}{ }^{-1}$ level, an elongated piece of emission is unambiguously seen within the cluster. XMM-Newton archival data (Obs. ID 0504630101, PI Andersson, $52.734 \mathrm{ks}, 0.1-12 \mathrm{keV})$, smoothed and exposure corrected, shows 

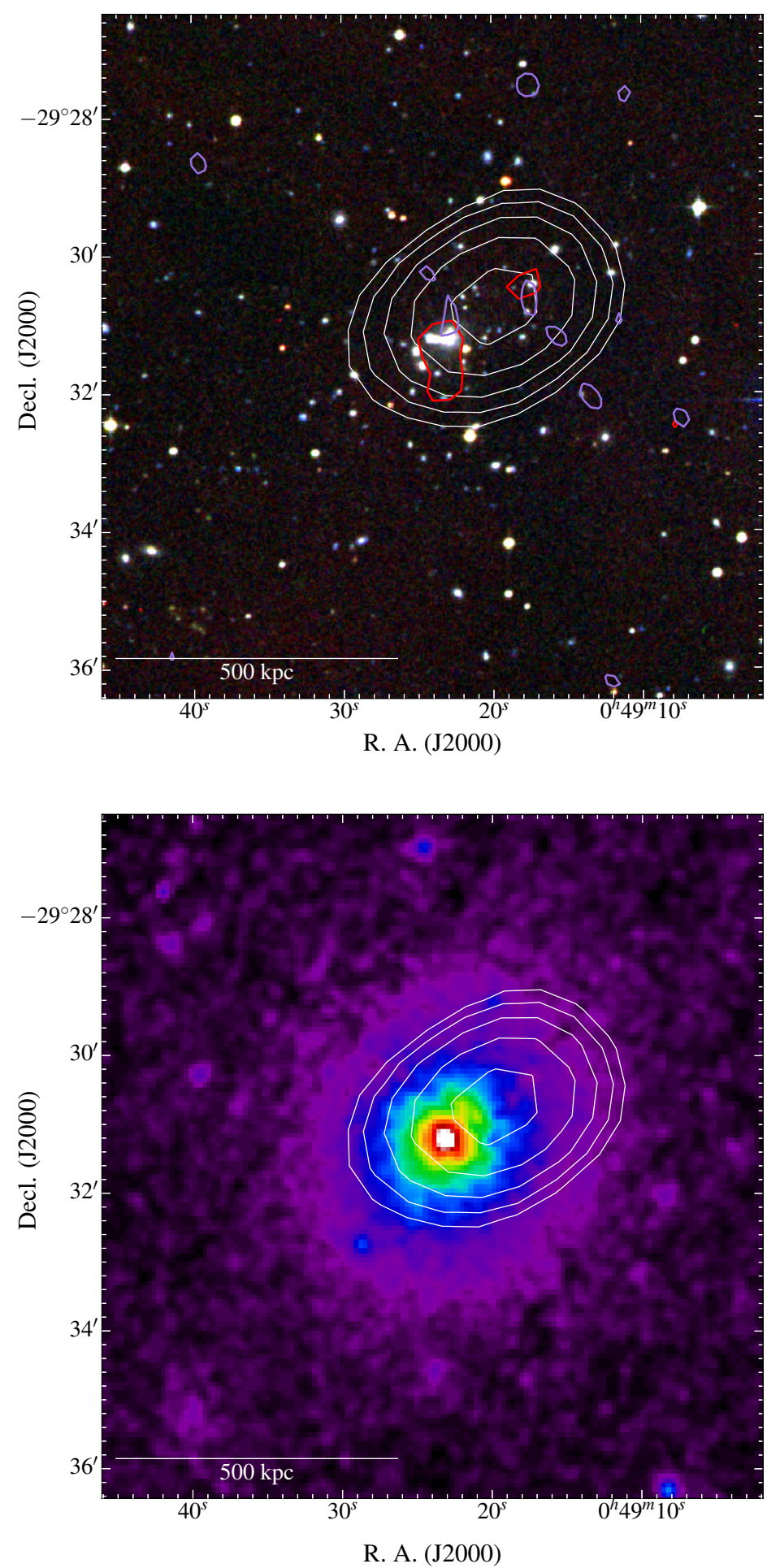

Figure ii.22: The centre of Abell S0084. Top: The background is an RGB image with contours overlaid as follows: EoR0, white, beginning at $7 \mathrm{mJy}_{\text {beam }}{ }^{-1}$ increasing by a factor of $\sqrt{2}$; NVSS, red, beginning at $1.5 \mathrm{mJy}_{\text {beam }}^{-1}$ increasing by a factor of 2; TGSS, medium purple, beginning at $12 \mathrm{mJy}_{\text {beam }}^{-1}$ also increasing by a factor of 2. Bottom: X-ray image from the REXCESS survey with EoR0 contours overlaid as in the top panel along with X-ray contours. 

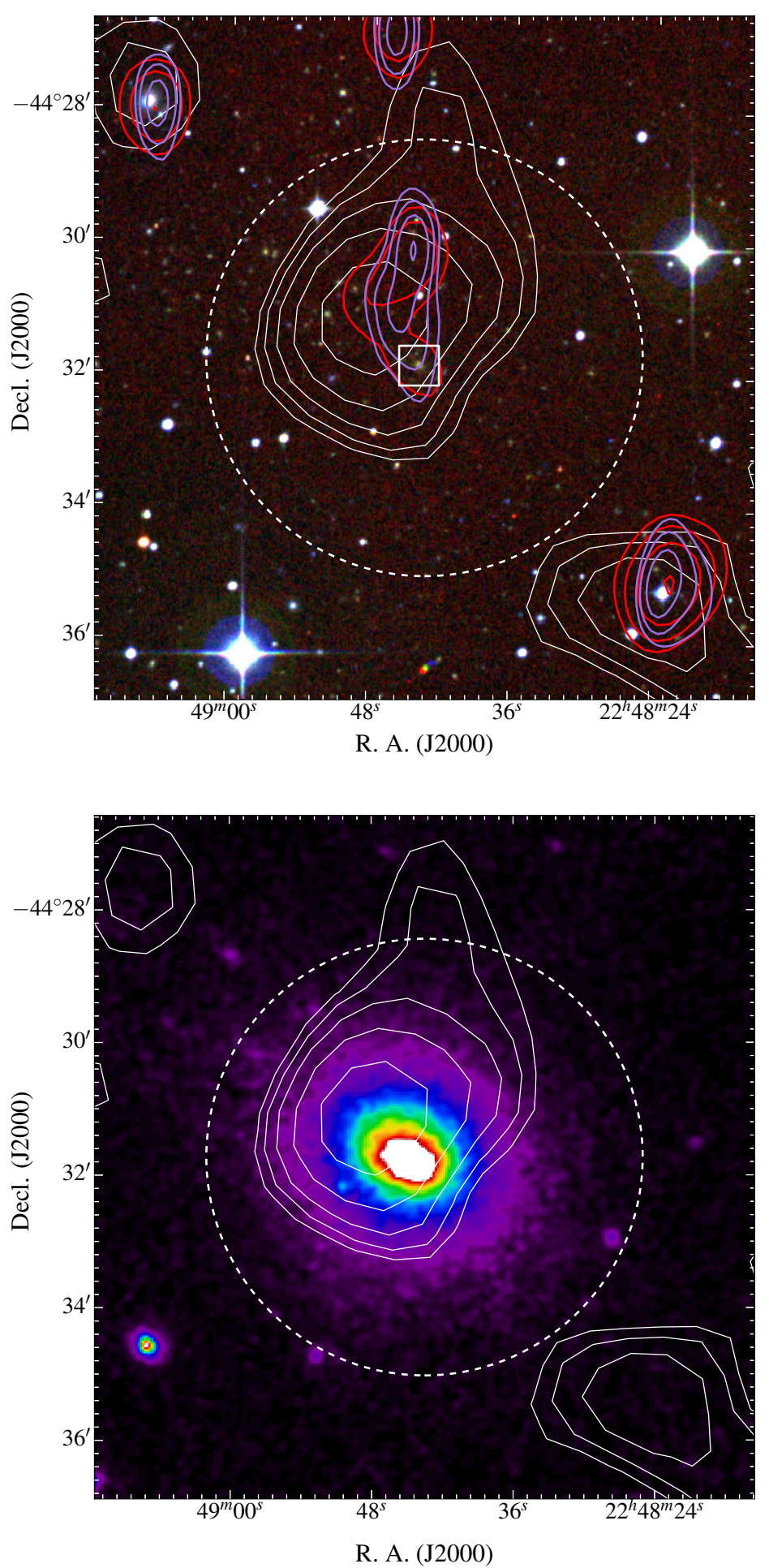

Figure ii.23: Abell S1063. Top: The background is an RGB image with contours overlaid as follows: EoR0, white, beginning at $50 \mathrm{mJy}_{\text {beam }}{ }^{-1}$ increasing by a factor of $\sqrt{2}$; SUMSS, red, beginning at $5 \mathrm{mJy}_{\text {beam }}{ }^{-1}$ increasing by a factor of 2 ; TGSS, medium purple, beginning at $7.8 \mathrm{mJy}^{\text {beam }}{ }^{-1}$ also increasing by a factor of 2. The dashed circle is centred on the cluster (with coordinates from Piffaretti et al. 2011 - see text) and has a radius of $1 \mathrm{Mpc}$. The white box indicates the BCG of the cluster. Bottom: The background image is the XMM-Newton data with EoR0 contours overlaid is in the top panel. 
strong X-ray emission coinciding with the $168 \mathrm{MHz}$ radio emission though their respective peaks lie offset to one another, with the X-ray peak situated at the position of the BCG (see Fig. ii.23). The X-ray emission can be seen to extend further northeast with the peak of the $168 \mathrm{MHz}$ emission occurring in this same direction. The BCG, LCRS B224549.3-444744 (with $z=0.347110 \pm 0.000250$; Guzzo et al. 2009) is marked with a square in Fig. ii.23. Note that the MCXC, PSZ1, and ACO catalogues all report different coordinates for the cluster centre, and it is the MCXC coordinates that coincide with the central BCG, hence the MCXC coordinates are taken as the cluster centre (e.g. in Fig. ii.23).

The emission at $168 \mathrm{MHz}$ has counterparts in the TGSS and SUMSS surveys. The morphology in the TGSS and SUMSS surveys is suggestive of a tailed radio galaxy, with the tail pointing into the cluster centre, however the peak of these TGSS and SUMSS emission does not show an optical ID for such a galaxy. The TGSS catalogue reports a source here with flux density $S_{147.5}=155.5 \pm 16.5 \mathrm{mJy}$ and the SUMSS catalogue has a source with $S_{843}=29.4 \pm 3.0 \mathrm{mJy}$. However, the source lies in a region where the TGSS ADR1 flux densities are systematically low, and so we do not consider the $147.5 \mathrm{MHz}$ flux density here. With the EoR0 field, a flux density (with a cut-off threshold of 50 mJy) of $S_{168}=265 \pm 38$ is measured. We also obtain an LAS of $\sim 5.5$ arcmin and an LLS of $\sim 1670 \mathrm{kpc}$. Taking the ratios of the logarithms of the 843 and $168 \mathrm{MHz}$ flux densities yields a spectral index for the source of $\alpha_{168}^{843}=-1.36 \pm 0.11$. Due to the difference in resolution, it is likely this is an upper limit, where a not insignificant percentage of the 843 $\mathrm{MHz}$ integrated flux density may be resolved out. This will likely be the case with the TGSS measurement of the source also, though it is hard to be certain with the inherent flux density discrepancy that exists already with the TGSS ADR1 data. We provide reduced, archival ATCA data and describe this cluster further in §iii.2.

\section{§ii.2.2.21. Abell S1099}

Abell S1099 is reported to have a redshift of $z=0.110400$ (Caretta et al., 2002). Fig. ii.24 shows the RGB image with MWA, NVSS, and TGSS contours overlaid. The cluster hosts extended, diffuse emission with steep spectral index, perhaps associated with one of the BCGs, 2MASX J23130574-2308369 ( $z=0.108575$; Caretta et al. 2004), which coincides with the peak of the emission at $168 \mathrm{MHz}$. We measure the $168 \mathrm{MHz}$ to be $S_{168}=184 \pm 20 \mathrm{mJy}$, where the uncertainty includes a term to account for the slight blending towards the northwestern source, NVSS J231317-230513. Further, Obj. A in Fig ii.24 appears to be an embedded point source, catalogued as NVSS J231255-230959 (Condon et al., 1998), which is not accounted for. We see that the $1.4 \mathrm{GHz}$ emission is patchy and use the MIRIAD (Sault et al., 1995) task CONVOL to convolve the NVSS image to the 108.87 arcsec $\times 108.87$ arcsec of the EoR0 field to measure the emission. This yields $S_{1.4}=22.3 \pm 6.4 \mathrm{mJy}$, where the uncertainty incorporates the embedded source and the blending with NVSS J231317-230513. We calculate a spectral index of $\alpha_{168}^{1.4}=-1.00 \pm 0.15$ which does not suggest a centrally located cluster 


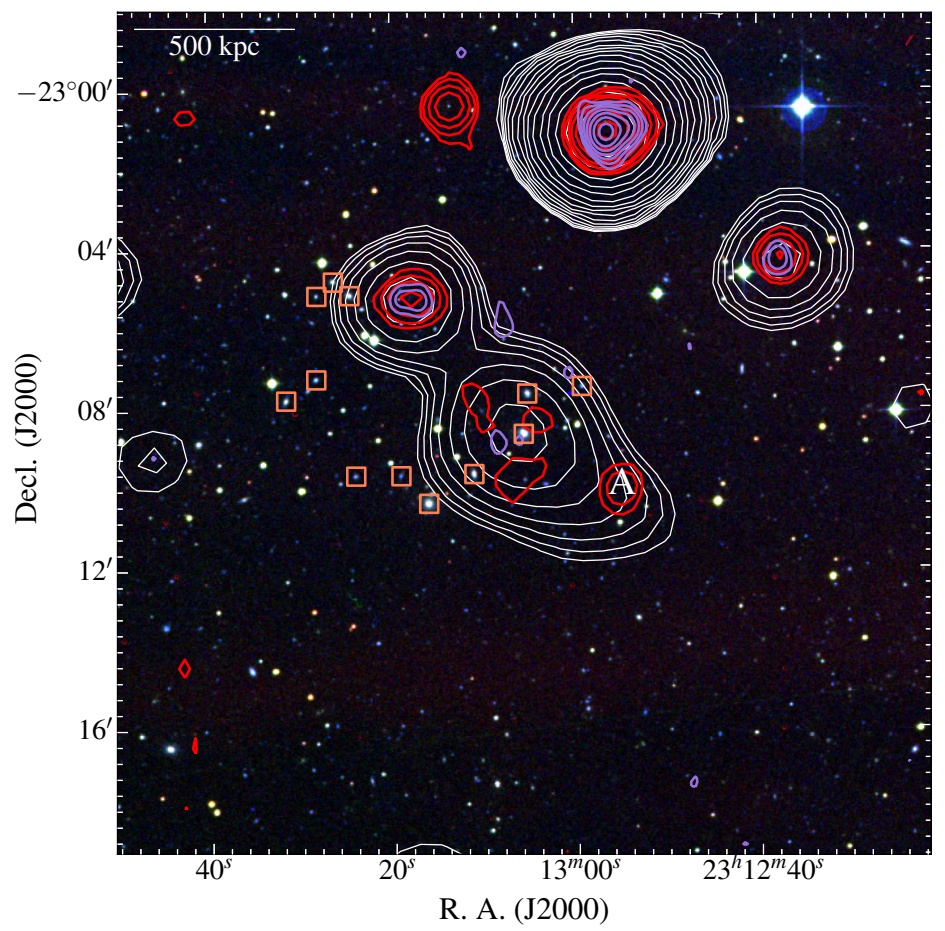

Figure ii.24: Diffuse emission in Abell S1099. The background is an RGB image with contours overlaid as follows: EoR0, white, beginning at $10 \mathrm{mJy}_{\text {beam }}{ }^{-1}$ and increasing with a factor of $\sqrt{2}$; NVSS, red, beginning at $1.5 \mathrm{mJy}^{\text {beam }}{ }^{-1}$ increasing by a factor of 2; TGSS, medium purple, beginning at $11.1 \mathrm{mJy} \mathrm{beam}^{-1}$ also increasing with a factor of 2. The linear scale is at the redshift of Abell S1099. "A" marks a likely embedded source.

relic, though is consistent with the flatter-spectrum haloes or peripheral relics. It is not clear where the cluster centre lies, and with no auxiliary X-ray data beyond RASS we are unable to determine if the emission is that of a cluster halo. The morphology, and apparent steep spectral index are suggestive of a halo, but the BCG at the centre of the emission and the lack of clear cluster centre casts doubt towards this classification.

\section{§ii.2.2.22. Abell S1121}

Abell S1121 (PSZ1 G348.92-67.38) is reported by Coziol et al. (2009) to have a redshift of $z=0.190431$ though Liu et al. (2015) report a redshift of $z=0.3580$ for this system. Assuming $z=0.3580$ as used by PSZ1 the cluster has a mass of $M_{\mathrm{YZ}, 500}=7.05_{-0.60}^{+0.61} \times 10^{14} M_{\odot}$ (PSZ1). The cluster hosts a radio halo, with extended $168 \mathrm{MHz}$ emission located at the cluster centre, with little-to-no SUMSS or TGSS counterparts at 843 and $147.5 \mathrm{MHz}$. There is a bright source to the north of the cluster that in SUMSS is creating artefacts streaking across the SUMSS image for this region. However, it is also important to note that Abell S1121 lies near the edge of the EoR0 field and is in an area of the image with higher noise, hence the $15 \mathrm{mJy}_{\text {beam }}{ }^{-1}$ contours in Fig. ii.25. The top panel of Fig. ii.25 

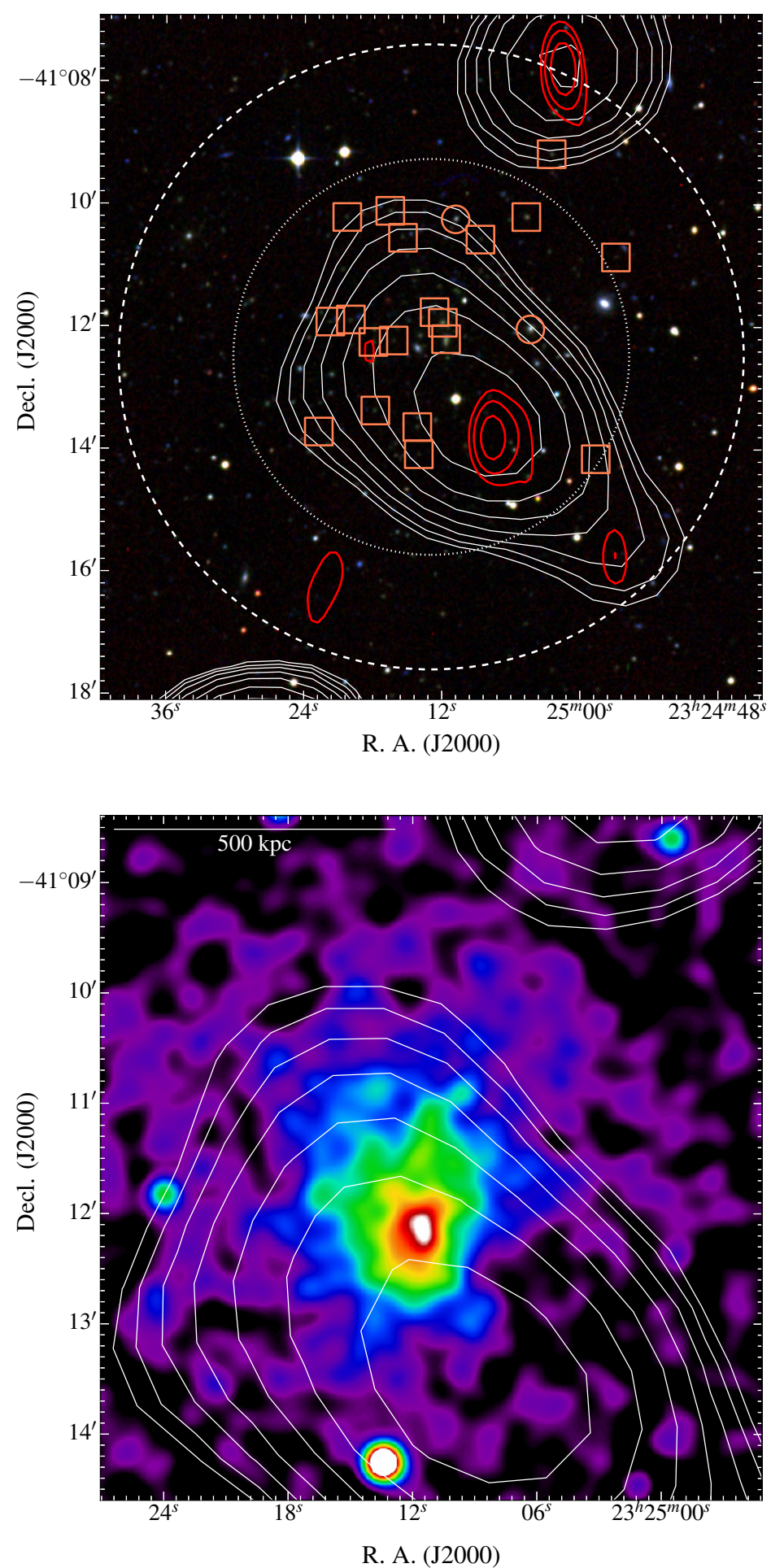

Figure ii.25: Candidate radio halo within Abell S1121. Top: The background is an RGB image with contours overlaid as follows: EoR0, $15 \mathrm{mJy}^{\text {beam }}{ }^{-1}$ increasing by a factor of $\sqrt{2}$ and TGSS, red, beginning at $13.8 \mathrm{mJy}^{-1}$ beam $^{-1}$ also increasing by a factor of 2 . The dashed and dotted circles are centred on the cluster with $1 \mathrm{Mpc}$ radii at each of the reported redshifts. The orange boxes and circles indicate galaxies with available redshifts within $2000 \mathrm{~km} \mathrm{~s}^{-1}$ at the two reported redshifts (see text) Bottom: Archival Chandra data smoothed to $\sim 15$ arcsec resolution with EoR0 contours as in the top panel along with X-ray contours. 
shows galaxies with available redshifts in the range $c z \approx 2000 \mathrm{~km} \mathrm{~s}^{-1}$ around the reported redshifts, with the orange circles associated with $z=0.190431$ and the orange boxes associated with $z=0.3580$. Given the location and numbers of each distribution, we consider the emission (and the cluster) to be at the redshift reported by Liu et al. (2015), $z=0.3580$. There is likely a separate, intervening system along the line-of-sight that Coziol et al. (2009) are measuring.

The bottom panel of Fig. ii.25 shows archival Chandra data (Obs. ID 13405, PI Garmire, exposure time $8.94 \mathrm{ks}, 0.1-13.1 \mathrm{keV}$ ). This X-ray emitting plasma is situated in the core of the cluster, however it has directionality, with morphology like a cone similar to the Bullet Cluster discussed along with Abell 0133. This type of morphology suggests cluster dynamics often coincident with radio haloes. The main component of the diffuse $168 \mathrm{MHz}$ emission coincides with this X-ray core. There are two point sources blended with the diffuse emission: SUMSS J232506-411339 and SUMSS J232457-411542. For SUMSS J232506-411339 we use the SUMSS catalogue measurement of $S_{843}=24.3 \pm 3.0 \mathrm{mJy}$ (Murphy et al., 2007) and assume a spectral index of $\alpha=-0.8 \pm 0.2$ to predict a $168 \mathrm{MHz}$ flux density of $S_{168}=88 \pm 31 \mathrm{mJy}$. Similarly, for SUMSS J232457-411542 we use the catalogue measurement of $S_{843}=11.6 \pm 2.1 \mathrm{mJy}$ (Murphy et al., 2007), also assuming a spectral index of $\alpha=-0.8 \pm 0.2$, to predict $S_{168}=42 \pm 16 \mathrm{mJy}$. The total flux of the emission is measured to be $S_{168}^{\text {tot }}=284 \pm 32 \mathrm{mJy}$. From this we estimate the flux density of the diffuse component to be $S_{168}=154 \pm 48 \mathrm{mJy}$. However, we have previously seen that extrapolating from poorly sampled SEDs - let alone using an assumed spectral index - does not always produce good results.

We estimate the LAS of the diffuse region of the emission to be $\sim 4.3$ arcmin which translates to an LLS of $\sim 1300 \mathrm{kpc}$ at $z=0.3580$. Given the location, size, coincidence with X-ray emission, approximate $168 \mathrm{MHz}$ flux density, and the fact that Abell S1121 is a reasonably massive cluster, we classify the newly detected emission as a giant radio halo.

\section{\$ii.2.2.23. Abell S1136}

Abell S1136 (MCXC J2336.2-3136) has a redshift of $z=0.0625$ (Schwope et al., 2000), X-ray luminosity $L_{500}=0.504007 \times 10^{37} \mathrm{~W}$, and mass $M_{\mathrm{X}, 500}=1.2886 \times 10^{14}$ $\mathrm{M}_{\odot}(\mathrm{MCXC})$. Fig. ii.26 shows the centre of the cluster with an elongated piece of diffuse radio emission appearing strongly at $168 \mathrm{MHz}$, with a patchy counterpart in the TGSS survey at 147.5 MHz. There is no corresponding $1.4 \mathrm{GHz}$ or $843 \mathrm{MHz}$ emission seen in the NVSS or SUMSS surveys. This implies a steep spectral index. The source to the west of the diffuse emission is a double source, catalogued as NVSS J233603-313431. We use AEGEAN once again, and see the diffuse source split into two distinct components. The total flux density of the diffuse source is then measured to be $S_{168}=586 \pm 46 \mathrm{mJy}$.

The RASS broad-band 0.1-2.4 keV image does not show particularly strong $\mathrm{X}$-ray emission at the centre, and the RGB image (Fig. ii.26) shows the optical concentration of galaxies at the centre is offset towards the west of the bulk of the 


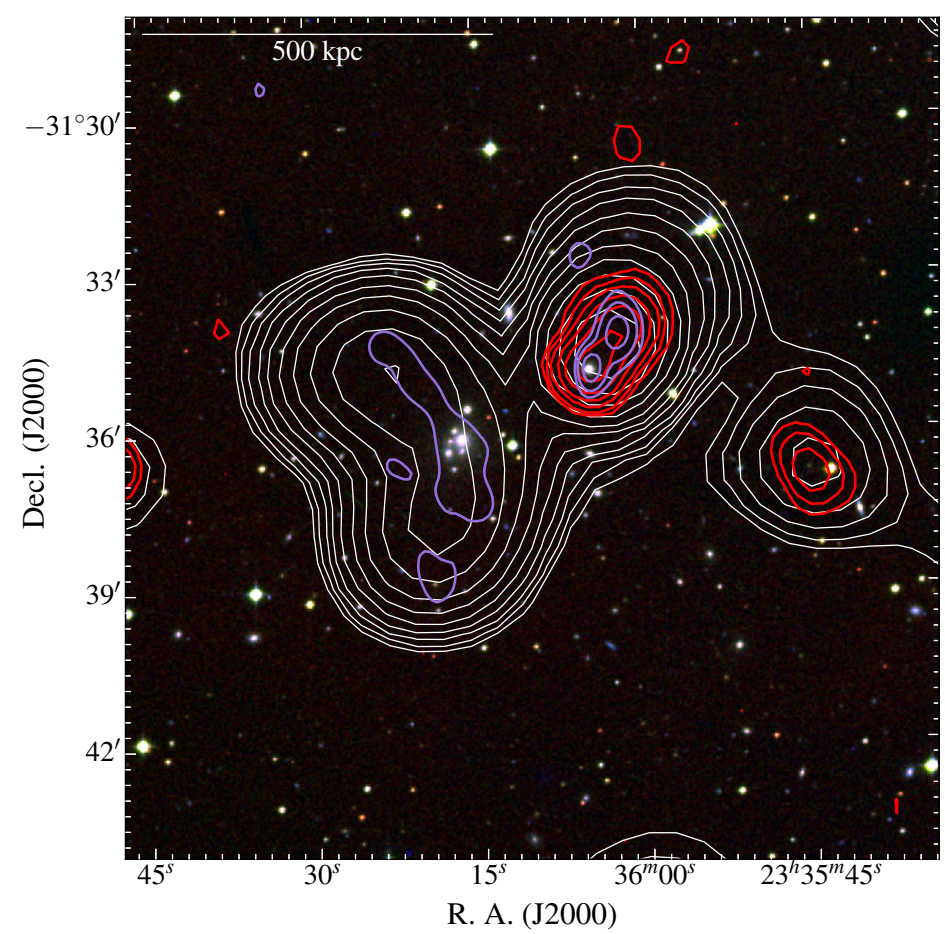

Figure ii.26: Diffuse emission within Abell S1136. The background is an RGB image with contours overlaid as follows: EoR0, white, beginning at $10 \mathrm{mJy}^{\text {beam }^{-1}}$ increasing by a factor of $\sqrt{2}$; NVSS, red, beginning at $1.5 \mathrm{mJy}^{\text {beam }}{ }^{-1}$ increasing by a factor of 2 ; TGSS, medium purple, beginning at 14.4 mJy beam $^{-1}$ also increasing by a factor of 2 .

$168 \mathrm{MHz}$ emission. The elongation is north-south, with an almost bent doublelobed structure, and has an LAS of $\sim 7.2$ arcmin which translates to an LLS of $\sim 530 \mathrm{kpc}$. While the emission could be classified as a cluster halo, alternate explanations are those of cluster relic intervening along the line-of-sight towards the cluster, or a dead radio galaxy likely having a previous association with the BCG, ESO 470-G020. Without polarisation data and higher resolution imaging we do not classify this emission here. A follow-up observation of the cluster with the ATCA is described in §iii.1.

\section{§ii.2.2.24. PSZ1 G307.55-77.87}

PSZ1 G307.55-77.87 is a distant cluster with a photometric redshift of $z=0.4530$ (Planck Collaboration et al., 2014) and mass of $M_{\mathrm{YZ}, 500}=5.69_{-0.88}^{+0.80} \times 10^{14} \mathrm{M}_{\odot}$ (PSZ1). Fig. ii.27 shows diffuse emission towards the cluster periphery. The nearby point source is LCRS B004346.8-393051 which has a redshift of $z=0.155664 \pm$ 0.000450 (Shectman et al., 1996) and is not associated with the cluster, though it may be the source of the diffuse emission within the context of a tail or lobe of a radio galaxy.

In the case that the emission is a cluster relic, we estimate the $168 \mathrm{MHz}$ flux density by estimating the $168 \mathrm{MHz}$ flux density of the LCRS source. The LCRS 


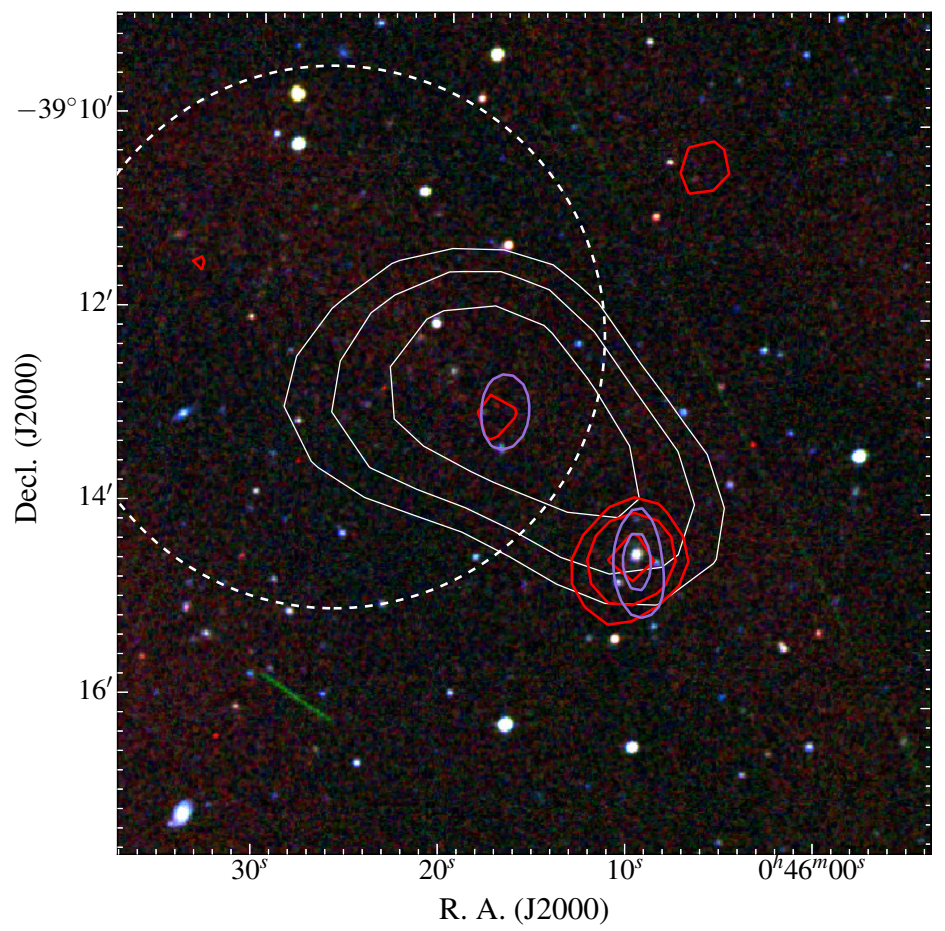

Figure ii.27: The cluster PSZ1 G307.55-77.87. The background is an RGB image with contours overlaid as follows: EoR0, white, beginning at $15 \mathrm{mJy}^{\text {beam }}{ }^{-1}$ increasing by a factor of $\sqrt{2}$; NVSS, red, beginning at $1.5 \mathrm{mJy}_{\text {beam }}{ }^{-1}$ increasing by a factor of 2; TGSS, medium purple, beginning at $7.5 \mathrm{mJy}_{\text {beam }}{ }^{-1}$ also increasing by a factor of 2 . The dashed circle is centred on the cluster with a radius of $1 \mathrm{Mpc}$.

source has 1.4 GHz flux density of $S_{1.4}^{\mathrm{LCRS}}=7.8 \pm 0.5 \mathrm{mJy}$ (Condon et al., 1998) and $843 \mathrm{MHz}$ flux density of $S_{843}^{\mathrm{LCRS}}=11.1 \pm 1.0 \mathrm{mJy}$ (Murphy et al., 2007) which give a spectral index for the LCRS source of $\alpha_{843}^{1.4}=-0.696 \pm 0.218$. Extrapolating from this and $S_{1.4}^{\mathrm{LCRS}}$ yields $S_{168}^{\mathrm{LCRS}}=34.4 \pm 16.2 \mathrm{mJy}$. The total emission of the possible relic and the LCRS source is measured to be $S_{168}^{\text {tot }}=52.7 \pm 12.7 \mathrm{mJy}$. This implies a flux density of $S_{168}=18 \pm 21 \mathrm{mJy}$ for the relic. Given the uncertainty there is not anything further we can say about the SED of the emission. However, the likely explanation is that of a radio galaxy and lobe or tail, not associated with the cluster but with LCRS B004346.8-393051.

\section{§ii.2.2.25. RXC J2351.0-1954}

RXC J2351.0-1954 (PSZ1 G057.09-74.45) has a redshift of $z=0.247700 \pm 0.000190$, a mass $M_{\mathrm{YZ}, 500}=5.60_{0.62}^{0.59} \times 10^{14} \mathrm{M}_{\odot}(\mathrm{PSZ} 1)$, and X-ray luminosity $L_{\mathrm{X}}=(4.33 \pm$ $0.84) \times 10^{37} \mathrm{~W}$ (Chon and Böhringer, 2012). The top panel of Fig. ii.28 shows the cluster and surrounding field, and the bottom panel shows the central region of the cluster. The dashed circle is at $1 \mathrm{Mpc}$ radius and centred on $\left(\alpha_{\mathrm{J} 2000}, \delta_{\mathrm{J} 2000}\right)=$ (357.7703, -19.9132). In the top panel of Fig. ii.28 two steep-spectrum, diffuse sources are located to the southeast (Obj. A) and northwest (Obj. B). The bottom panel of the same figure shows a zoomed-in view of the cluster centre. 

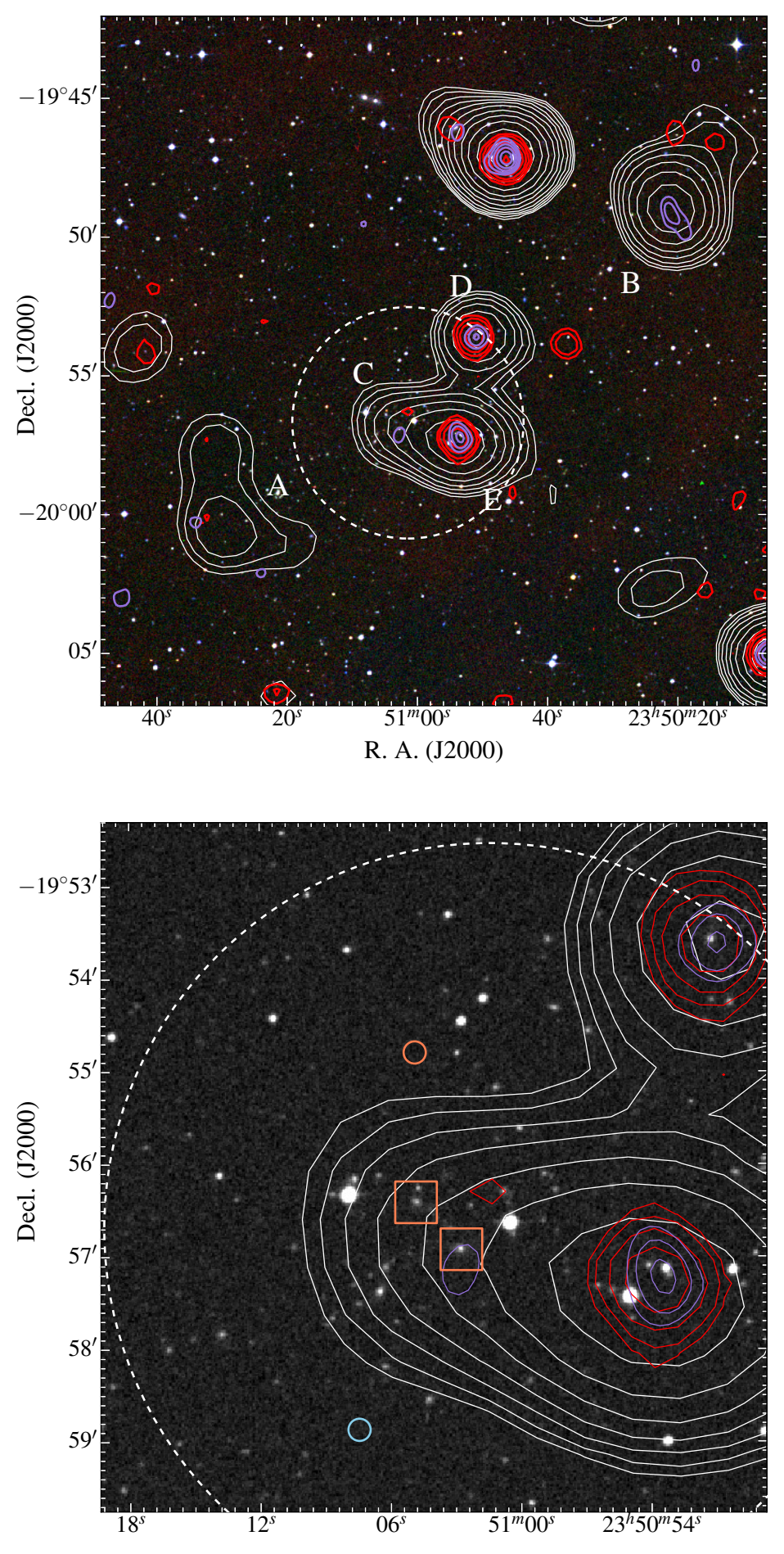

R. A. (J2000)

Figure ii.28: RXC J2351.0-1954. Top: RGB image with contours overlaid as follows: EoR0, white, beginning at $10 \mathrm{mJy}^{-1} \mathrm{beam}^{-1}$ and increasing with a factor of $\sqrt{2}$; NVSS, red, beginning at $1.5 \mathrm{mJy}^{\text {beam }}{ }^{-1}$ and increasing with a factor of 2 ; TGSS, medium purple, beginning at $13.8 \mathrm{mJy}_{\text {beam }}^{-1}$ also increasing with a factor of 2 . The dashed circle is centred on the cluster coordinates with a radius of $1 \mathrm{Mpc}$. Bottom: A smaller field of view of the top panel using the DSS2 red band only, with a small orange circle to denote the cluster's coordinates given by Chon and Böhringer (2012) and orange squares to show galaxies with spectroscopic redshifts in the region. 
Table ii.4: $168 \mathrm{MHz}$ flux densities and spectral indices calculated for blended sources near RXC J2351.0-1954.

\begin{tabular}{lcc}
\hline \hline Source & $\begin{array}{c}S_{168} \\
(\mathrm{mJy})\end{array}$ & $\alpha_{147.5}^{1.4}$ \\
\hline APMUKS(BJ) B234816.36-201016.8 (D) & $78.6 \pm 10.7$ & $-0.50 \pm 0.06$ \\
NVSS J235053-195715 (E) & $56.5 \pm 7.9$ & $-0.59 \pm 0.06$ \\
Central diffuse emission (C) & $87 \pm 17$ & - \\
\hline
\end{tabular}

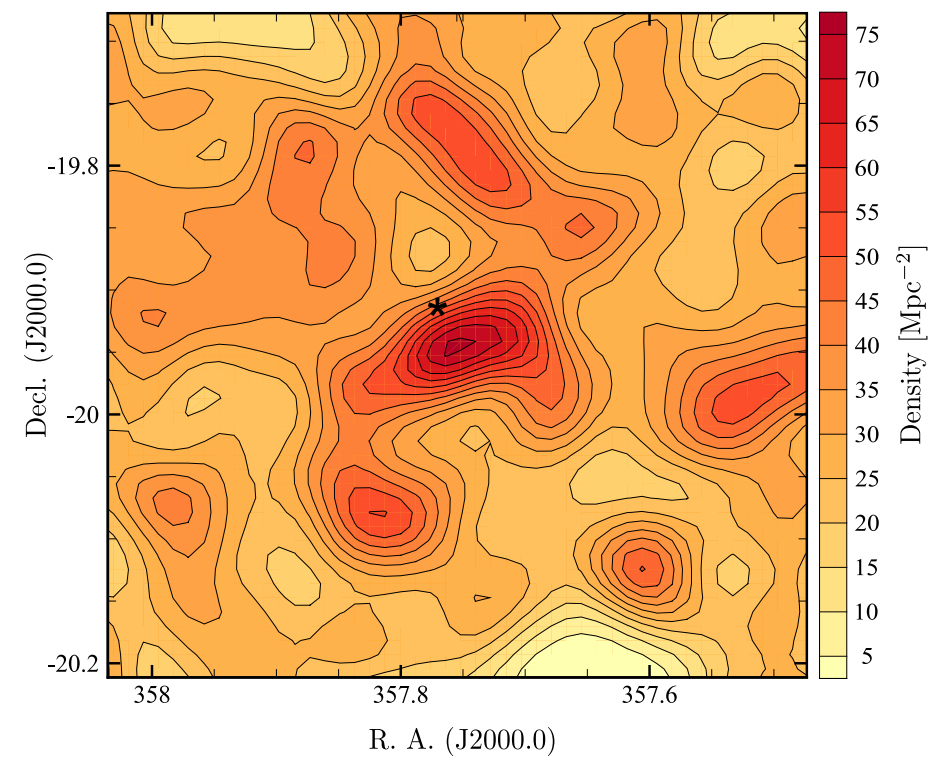

Figure ii.29: Optical density map of the area surrounding RXC J2351.0-1954. The asterisk marks the coordinates of the cluster given by Chon and Böhringer (2012). This map was generated by Dr. Siamak Dehghan.

Obj. A and B have similarities with double relics on the periphery of merging clusters. Obj. A is $\sim 8.35$ arcmin $(\sim 2 \mathrm{Mpc})$ from the cluster centre and Obj. B is $\sim 11.8$ arcmin $(\sim 2.8 \mathrm{Mpc})$. Neither A nor B have an obvious ID visible in the blue, red, or IR bands from the DSS2. The flux densities of the sources are measured to be $S_{168}^{\mathrm{A}}=56.9 \pm 8.6 \mathrm{mJy}$ and $S_{168}^{\mathrm{B}}=147 \pm 13 \mathrm{mJy}$. Neither source appears in the NVSS data at $1.4 \mathrm{GHz}$ so we place upper limits on the $1.4 \mathrm{GHz}$ flux densities of $S_{1.4}^{\mathrm{A}} \leq 4.22$ and $S_{1.4}^{\mathrm{B}} \leq 4.7 \mathrm{mJy}$ places upper limits on the spectral indices of $\alpha_{168}^{1.4, \mathrm{~A}} \leq-1.23 \pm 0.07$ and $\alpha_{168}^{1.4, \mathrm{~B}} \leq-1.68 \pm 0.04$. Obj. B shows $147.5 \mathrm{MHz}$ emission in the TGSS image above $3 \sigma_{\text {rms }}$ but is not part of the TGSS ADR1 catalogue as it is not detected above $7 \sigma_{\text {rms }}$. Further, emission from Obj. B is likely resolved out of the TGSS image due to its higher resolution than the MWA. Obj. A shows the elongated, bent shape that is typical of cluster relics. Obj. B shows a more regular morphology at $168 \mathrm{MHz}$ in the EoR0, though shows elongation similar to a radio galaxy in the TGSS data.

The bottom panel of Fig. ii.28 shows the cluster centre, with the small, or- 
ange circle indicating the central coordinates provided by Chon and Böhringer (2012). Coordinates provided by Planck Collaboration et al. (2015a) lie to the southeast. The red-band DSS2 image shows an optical concentration of galaxies coinciding with $168 \mathrm{MHz}$ radio emission extending from the point source NVSS J235053-195715. The orange squares indicate the positions of galaxies with spectroscopic redshifts, at the approximate redshift of the cluster and reported by Chon and Böhringer (2012). We assume that the centre of the cluster as traced by the optically visible galaxies, which does not coincide with coordinates from Chon and Böhringer. To better define the cluster centre, Dr. Siamak Dehghan produced Fig ii.29, which shows the isodensity map generated from 2972 galaxies within half a degree of the cluster's reported centre, obtained from the SuperCOSMOS Sky Survey (Hambly et al., 2001c,b,a). The contour map was made using a grid of $150 \mathrm{kpc}$ cells (at the redshift of the cluster) and then smoothed via a Gaussian kernel with standard deviation of $250 \mathrm{kpc}$. The density peak at $\alpha_{\mathrm{J} 2000}=357.756$ and $\delta_{\mathrm{J} 2000}=-19.945$ is about 2 arcmin from the reported centre of the cluster. We consider these peak-density coordinates as the cluster centre, which still disagree with the coordinates provided by PSZ1.

The $168 \mathrm{MHz}$ emission enveloping those galaxies at the optical centre of the cluster is likely a cluster halo, with the lack of significant $1.4 \mathrm{GHz}$ NVSS emission implying a steep-spectrum source. We estimate the flux density of this diffuse emission by subtracting the extrapolated flux densities of the two blended sources, APMUKS(BJ) B234816.36-201016.8 and NVSS J235053-195715 (Obj. D and E in Fig. ii.28), using $1.4 \mathrm{GHz}$ and $147.5 \mathrm{MHz}$ flux density measurements from the NVSS and TGSS catalogues respectively (Condon et al., 1998; Intema et al., 2016). The spectral indices and extrapolated $168 \mathrm{MHz}$ flux densities are presented in Table ii.4. The diffuse source is estimated to have a flux density of $S_{168}=87 \pm 17 \mathrm{mJy}$. From the lack of $1.4 \mathrm{GHz}$ emission in the NVSS image above $3 \sigma_{\text {rms }}$ we estimate an upper limit of $S_{1.4} \leq 4.3 \mathrm{mJy}$ resulting in an upper limit of $\alpha_{168}^{1.4} \leq-1.4 \pm 0.1$ for the spectral index. No significant emission is seen in the RASS broad band image, however due to the cluster's redshift this is not surprising. Without supplementary archival Chandra or XMM-Newton data it is difficult to properly classify this emission as a halo. We consider this a newly detected candidate radio halo.

\section{§ii.2.2.26. MACS J2243.3-0935}

Cantwell et al. (2016) report the detection of a radio halo in the merging cluster MACS J2243.3-0935 (MCXC J2243.3-0935; PSZ1 G056.94-55.06), detected using the Karoo Array Telescope-7 telescope and GMRT. The cluster has a redshift of $z=0.447$ (Ebeling et al., 2010), mass $M_{\mathrm{YZ}, 500}=10.07_{-0.60}^{+0.58} \times 10^{14} M_{\odot}(\mathrm{PSZ} 1)$, and X-ray luminosity $L_{500}=15.200000 \times 10^{37} \mathrm{~W}(\mathrm{MCXC})$. Fig. ii.30 shows the the MWA contours overlaid on the RGB image. MACS J2243.3-0935 is near the edge of the EoR0 field and so the region within which it resides features heavy noise. Because of this, the detection is tentative, with the emission barely showing above $2 \sigma_{\text {rms }}$. Fig. ii.30 shows the cluster with $2 \sigma_{\text {rms }}$ contours to emphasise this. 


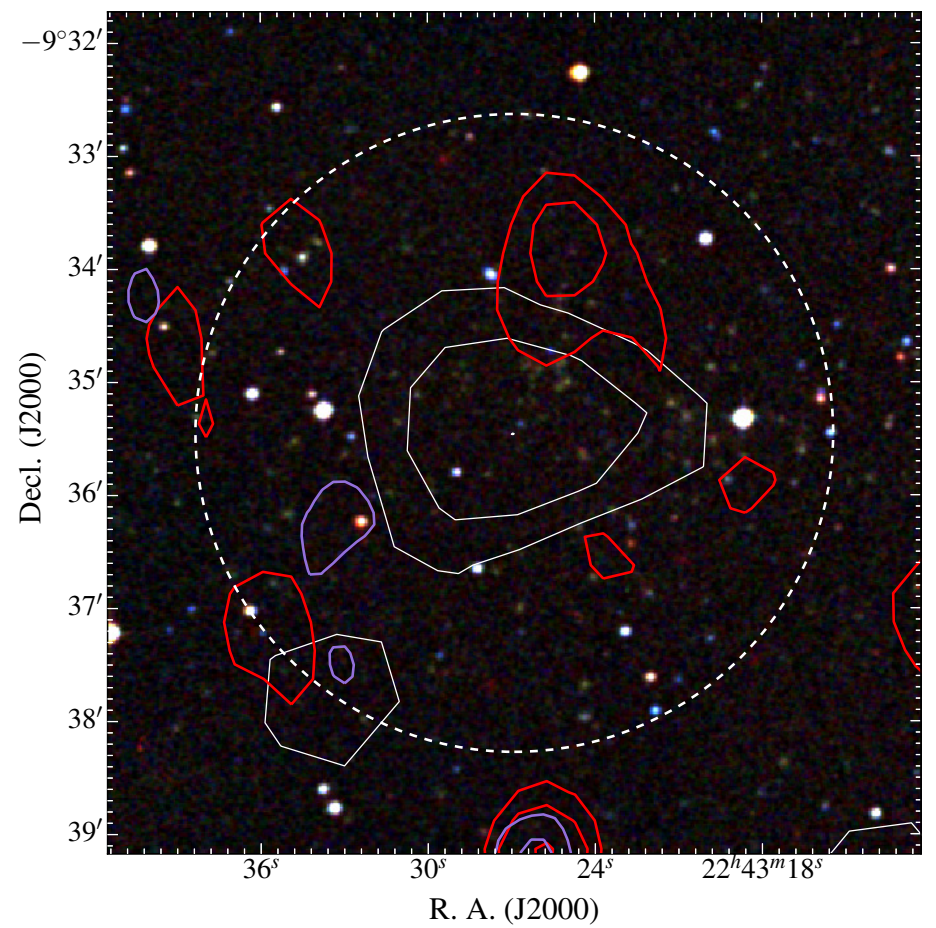

Figure ii.30: MACS J2243.3-0935 with radio halo. The background is an RGB image with contours overlaid as follows: EoR0, white, beginning at $60 \mathrm{mJy}$ beam $^{-1}$ increasing by a factor of $\sqrt{2}$; NVSS, red, beginning at $1.5 \mathrm{mJy}_{\text {beam }}{ }^{-1}$ increasing by a factor of 2; TGSS, medium purple, beginning at $10.2 \mathrm{mJy}_{\text {beam }}^{-1}$ also increasing by a factor of 2 . The dashed circle is centred on the cluster with a radius of $1 \mathrm{Mpc}$. 


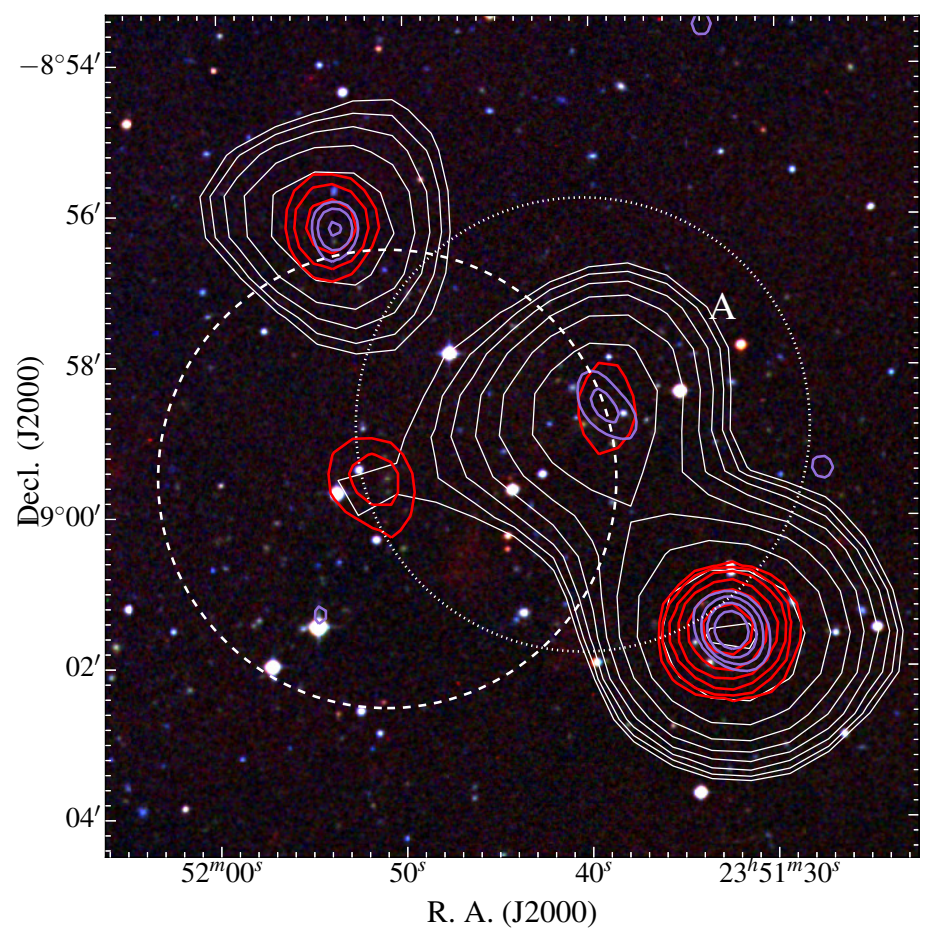

Figure ii.31: Diffuse emission on the periphery of WHL J235151.0-085929 or centre of GMBCG J357.91841-08.97978. The background is an RGB image with contours overlaid as follows: EoR0, white, beginning at $10 \mathrm{mJy} \mathrm{beam}^{-1}$ increasing by a factor of $\sqrt{2}$; NVSS, red, beginning at $1.5 \mathrm{mJy}^{\text {beam }}{ }^{-1}$ increasing by a factor of 2 ; TGSS, medium purple, beginning at $11.7 \mathrm{mJy}_{\text {beam }}^{-1}$ also increasing by a factor of 2 . The dashed circle is centred on the PSZ1 coordinates of WHL J235151.0-085929 with radius $1 \mathrm{Mpc}$. The dotted circle is centred on the cluster GMBCG J357.9184108.97978 with the same $1 \mathrm{Mpc}$ radius.

At this level we measure the $168 \mathrm{MHz}$ flux density to be $S_{168}=83 \pm 36 \mathrm{mJy}$, with an LAS of $\sim 2.8$ arcmin and a corresponding LLS of $\sim 1$ Mpc. With the 610 MHz flux density measured by Cantwell et al. (2016) we obtain a spectral index of $\alpha_{168}^{610}=-1.6 \pm 0.4$. However, these results should be taken with caution due to the noise in this region of the EoR0 field.

\section{§ii.2.2.27. WHL J235151.0-085929}

WHL J235151.0-085929 (PSZ1 G082.31-67.01), a distant cluster with a redshift of $z=0.3939$ (Wen et al., 2012) and mass $M_{\mathrm{YZ}, 500}=5.90_{-0.84}^{+0.78} \times 10^{14} \mathrm{M}_{\odot}(\mathrm{PSZ} 1)$. Fig. ii.31 shows the cluster with an RGB (red, IR, blue) background and radio contours overlaid. Obj. A is a possible diffuse source on the cluster's periphery. The cluster does not show significant X-ray emission in the RASS broad-band image. The location of the emission relative to the cluster centre and the lack of optical ID (see Fig. ii.31) are suggestive of a cluster relic. We note that source is point-like in the EoR0 field image, and that at this resolution-especially given the redshift of the cluster - there is an inherent uncertainty in whether the source 
is extended or not. The NVSS image shows a source that is extended in the north-south direction, beyond that of the beam which is circular. The source does not appear in the TGSS ADR1 catalogue due to their higher $7 \sigma_{\text {rms }}$ cutoff but is present in the NVSS catalogue, with $1.4 \mathrm{GHz}$ flux density of $S_{1.4}=4.1 \pm 0.6$ mJy (Condon et al., 1998). We use AEGEAN here to measure the flux density of the source to be $S_{168}=128 \pm 20 \mathrm{mJy}$. This, in conjunction with the $1.4 \mathrm{GHz}$ measurement, is used to estimate a spectral index of $\alpha_{168}^{1.4}=-1.62 \pm 0.10$.

We do however note that the Gaussian Mixture Brightest Cluster Galaxy (GMBCG; Hao et al., 2010) catalogue reports a cluster at the centre of the emission: GMBCG J357.91841-08.97978, with a photometric redshift of $z=0.4$. It is entirely likely that the emission resides within this cluster. If this is the case the steep spectral index and central location would imply a cluster halo. The two clusters, WHL J235151.0-085929 and GMBCG J357.91841-08.97978, have centres separated by $\sim 2.7$ arcmin which at $z=0.3939$ is $\sim 890 \mathrm{kpc}$. This separation in both angular distance and redshift would suggest either the clusters are interacting or that they are the same cluster. With this in mind we suggest that the emission is a candidate cluster halo, at a redshift of $z=0.3939$, associated with the cluster GMBCG J357.91841-08.97978.

\section{§ii.2.3. On the scaling relations of cluster radio haloes}

\section{§ii.2.3.1. The $P_{1.4}-L_{\mathrm{X}}$ relation}

There exists an empirical relation between the thermal and non-thermal emission of galaxy clusters traced by the synchrotron emission giving the radio halo $1.4 \mathrm{GHz}$ power, $P_{1.4}$ and the thermal Bremsstrahlung X-ray emission giving the cluster $\mathrm{X}$ ray luminosity, $L_{\mathrm{X}}$. The $P_{1.4}-L_{\mathrm{X}}$ scaling relations have been studied and added too as each new halo detection or halo survey is released (e.g. GRHS: I; Venturi et al. 2007 and II; Venturi et al. 2008, EGRHS ${ }^{7}$ : I; Kale et al. 2013 and II; Kale et al. 2015, KAT-7 observations: Bernardi et al. 2016, ARDES: Shakouri et al. 2016) in an attempt to improve understanding about how the thermal X-ray emitting plasma interacts with the synchrotron electron population and how these relations might be caused by the clusters' often dynamical natures.

For those haloes in our sample with measured $168 \mathrm{MHz}$ flux densities and X-ray luminosities, we extrapolate using an assumed spectral index of $\alpha=-1.3 \pm 0.2$ (e.g. Cassano et al. 2013 use -1.3 for haloes with no spectral index) to obtain the $1.4 \mathrm{GHz}$ flux densities. We do not discriminate between ultra-steep spectrum and normal-spectrum radio haloes, as a number of the new detections presented here fall within the ultra-steep category when considering their spectral index limits. We then obtain the $k$-corrected $1.4 \mathrm{GHz}$ radio power, $P_{1.4}$, (see Hogg,

\footnotetext{
${ }^{6}$ Adapted from https://github.com/rsnemmen/nemmen/blob/master/nemmen/stats. py

${ }^{7}$ Extended GMRT Radio Halo Survey
} 


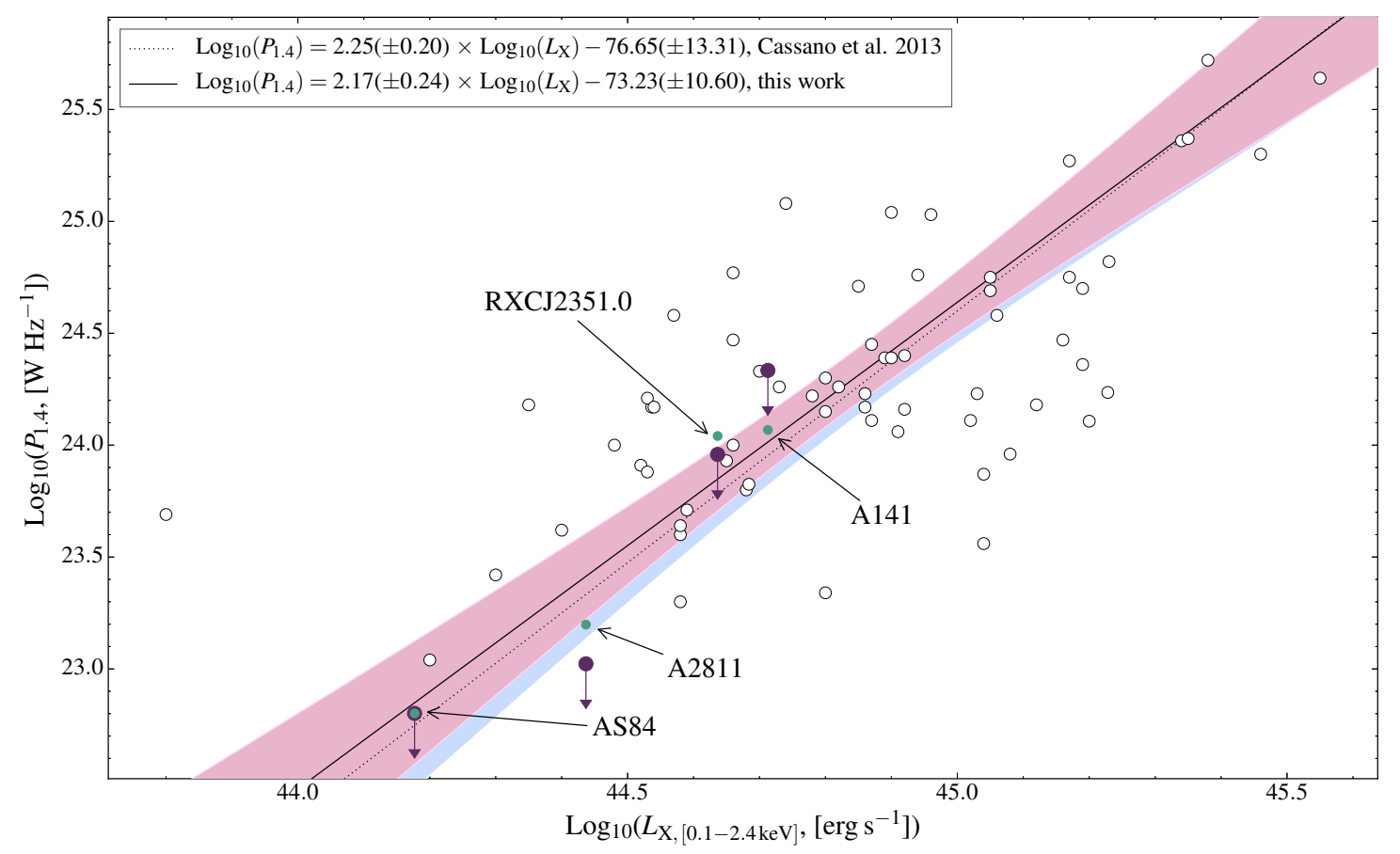

Figure ii.32: The $P_{1.4}-L_{\mathrm{X}}$ scaling relation updated with the halo detections in this paper. Error bars have been omitted for the sake of clarity. The black unfilled circles are haloes from the literature, drawing from the same sample as Shakouri et al. (2016) with the addition of the haloes detected in Triangulum Australis (Scaife et al., 2015), MACS J2243.3-0935 (Cantwell et al., 2016), ACT-CL J0256.5+0006 (Knowles et al., 2016), and PSZ1 G285.0-23.7 (Martinez Aviles et al., 2016). The green, filled circles are new halo detections from this paper, with assumed spectral indices of $\alpha=-1.3 \pm 0.2$. The dark-purple, filled circles are the same new halo detections but with the spectral index limits calculated here, thus they are upper limits to the $1.4 \mathrm{GHz}$ radio halo power. For X-ray luminosities and radio power measurements without quoted uncertainties we assume a standard three per cent uncertainty to allow for BCES fitting (see text). The mauve shaded region represents a 95 per cent confidence interval ${ }^{6}$ associated with the solid black BCES orthogonal fit made to all the data. The powder-blue region is the same for the dotted BCES bisector fit presented by Cassano et al. (2013). The halo associated with the cluster CL $0217+70$ has been included. Note that radio power is computed here assuming a flat $\Lambda \mathrm{CDM}$ cosmology, with $H_{0}=70 \mathrm{~km} \mathrm{~s}^{-1} \mathrm{Mpc}^{-1}, \Omega_{m}=0.3$, and $\Omega_{\Lambda}=0.7$, as compared to the rest of §ii which uses $H_{0}=68 \mathrm{~km} \mathrm{~s}^{-1} \mathrm{Mpc}^{-1}$. 
1999; Hogg et al., 2002) via

$$
P_{1.4}=\frac{4 \pi D_{\mathrm{L}}^{2}(z)}{(1+z)^{1+\alpha}} S_{1.4} \quad\left[\mathrm{~W} \mathrm{~Hz}^{-1}\right]
$$

with the luminosity distance, $D_{\mathrm{L}}(z)$, at the cluster's redshift, with associated error, $\sigma_{P_{1.4}}$,

$$
\sigma_{P_{1.4}}=\frac{4 \pi D_{\mathrm{L}}^{2}(z)}{(1+z)^{1+\alpha}} \sqrt{\left[S_{1.4} \ln (1+z) \sigma_{\alpha}\right]^{2}+\left(\sigma_{S_{1.4}}\right)^{2}} \quad\left[\mathrm{~W} \mathrm{~Hz}^{-1}\right] .
$$

Further, for radio haloes in our sample with spectral index limits we calculate upper limits to the $P_{1.4}$ values. Fig. ii.32 shows the 64 current literature halo detections with $L_{\mathrm{X}}$ measurements along with the newly detected halo in Abell 0141 and the newly detected candidate haloes in Abell 0141, Abell 2811, Abell S0084, and RXC J2351.0-1954. All but three (Triangulum Australis, MACS J2243.3-0935, and ACT-CL J0256.5+0006) of the previous literature detections are included by Shakouri et al. (2016) in their scaling relation plots. For Triangulum Australis we use $L_{\mathrm{X},[0.1-2.4 \mathrm{keV}]}=(4.83 \pm 0.12) \times 10^{44} \mathrm{erg} \mathrm{s}^{-1}$ (Zhao et al., 2013). The cluster Abell 1213 has been removed from our sample as we do not consider this emission to be that of a cluster halo. The diffuse emission detected in Abell 1213 has an LLS of $\sim 250 \mathrm{kpc}$ (LAS $\sim 4.3 \mathrm{arcmin}$ ), and is asymmetric. It is more likely a radio phoenix within our classification scheme, or possibly a mini-halo. Further, as a point of difference from values presented by Shakouri et al. (2016), for Abell 0523 we use the X-ray luminosity estimated by Girardi et al. (2016) of $3.44 \times 10^{44} \mathrm{erg} \mathrm{s}^{-1}$ compared to $1.07 \times 10^{44} \mathrm{erg} \mathrm{s}^{-1}$ reported by Ebeling et al. (1998).

For $L_{\mathrm{X}}$ and $P_{1.4}$ measurements without quoted uncertainties we attribute a standard three per cent error, which is approximately the mean value of the reported uncertainties, such that we can utilise the Bivariate Correlated Errors and intrinsic Scatter (BCES) linear regression method (Akritas and Bershady, 1996) to determine the best-fitting parameters to the data. We use the PYTHON code BCES ${ }^{8}$, a utilisation of which is shown by Nemmen et al. (2012). Table ii.5 summarises the best-fitting parameters for each of the BCES methods. Fig. ii.32 shows the best-fitting line (solid black) of the BCES orthogonal method, with the mauve, shaded region the 95 per cent confidence levels for that fit. The fit shown in Fig. ii.32 uses the assumed $\alpha=-1.3 \pm 0.2$ rather than the upper limits calculated. We also estimate the raw scatter, $\sigma_{\text {raw }}$, in the data via the error-weighted orthogonal distances to the best-fitting regression line via (e.g. Pratt et al., 2009; Cassano et al., 2013)

$$
\sigma_{\text {raw }}^{2}=\frac{(N / N-2)}{\sum_{i=1}^{N} 1 / \sigma_{i}^{2}} \sum_{i=1}^{N} \frac{1}{\sigma_{i}^{2}}\left(Y_{i}-a X_{i}-b\right),
$$

where $N$ is the sample size, $\sigma_{i}{ }^{2}=\sigma_{y_{i}}^{2}+a^{2} \sigma_{x_{i}}^{2}$ for uncertainties $\sigma_{y}, \sigma_{x}$ in $Y, X$, and fitting parameters $a, b$.

\footnotetext{
${ }^{8}$ Available at https://github.com/rsnemmen/BCES
} 
We find that the equivalent BCES orthogonal fit from Cassano et al. (2013) is consistent within the uncertainties of the data, however the raw scatter for equivalent fits has increased with the increase in sample size. New haloes and halo candidates from this work lie where expected. The halo within Abell S0084 is hosted by the second least X-ray luminous cluster in the sample, surpassed only by CL $0217+70$; its location on the $P_{1.4}-L_{\mathrm{X}}$ diagram expected for the cluster's X-ray luminosity assuming the spectral index is close to -1.3 . Such a lowluminosity, low-power detection was expected of the MWA due to its sensitivity to weak, steep-spectrum emission. However, we would also expect to find more ultra-steep spectrum halo candidates, given the frequency of $168 \mathrm{MHz}$ used here. Only one halo in our sample clearly fits this definition: the candidate halo within GMBCG J357.91841-08.97978 with $\alpha_{168}^{1400}=-1.61 \pm 0.10$. This cluster, however, does not have a measured X-ray luminosity, nor significant emission seen in the soft-band RASS image, thus is not included in the present analysis.

There is a single halo that lies far beyond the rest of the sample, residing in the under-luminous cluster CL $0217+70$, detected via its radio emission (Brown et al., 2011). The cluster is somewhat unique in its detection via radio emission, but also in that it features both a halo and a pair of relics. Its nature as under-luminous in the $0.1-2.4 \mathrm{keV} \mathrm{X}$-ray band may be an effect of an uncertain redshift, though we have included the halo assuming the redshift is correct. Table ii.5 includes BCES best-fitting parameters for the four BCES methods, both including and excluding CL $0217+70$. We see that the raw scatter $\left(\sigma_{\text {raw }}\right)$ of the data is lower when excluding CL $0217+70$, and that the overall fits tend to be steeper.

\section{§ii.2.3.2. The $P_{1.4}-M_{500}$ relation}

We also update the known $P_{1.4}-M_{500}$ scaling relation (e.g. Cassano et al. 2013, Shakouri et al. in prep.) similarly. We draw again from the same sample of haloes of Shakouri et al. (2016, in prep.) with the exception of the following clusters: Abell 1213, Abell 2218, as we do not consider these haloes; CL 0217+70, CL 1446+26, Abell 339, Abell 1550, due to lack of $M_{500}$ value; we do not use Abell 0851 as mass estimates through various methods vary from $4.4 \times 10^{14} \mathrm{M}_{\odot}$ (Martinet et al., 2016) to $12.5 \times 10^{14} \mathrm{M}_{\odot}$ (Hoekstra et al., 2015); and we do not use Abell 0800 as the only available mass is that given as part of the MCXC which uses $\mathrm{X}$-ray luminosity as the mass proxy compared to the Compton parameter, $Y_{\mathrm{SZ}}$, as used by PSZ1. For the remaining clusters, mass estimates are taken from PSZ1 except in the cases of MACS J0416.1-2403 (Umetsu et al., 2014) and Abell 0523 (Girardi et al., 2016). We also add the newly detected haloes in Abell S1121 and Abell 0141, and the candidate haloes in Abell 2811, Abell S0084, RXC J2351.01954, and WHL J235151.0-085929, as well as the recent detections in Triangulum Australis, MACS J2243.3-0935, ACT-CL J0256.5+0006, and PSZ1 G285.0-23.7.

Fig. ii.33 shows the updated scaling relation. We use the BCES method again to determine best-fitting parameters to the data. Table ii. 5 summarises the bestfitting parameters for the four BCES methods for both the $P_{1.4}-L_{\mathrm{X}}$ and the $P_{1.4}{ }^{-}$ 
Table ii.5: Best-fitting parameters from the BCES fitting with different methods, where $\log _{10}\left(P_{1.4}\right)=a \log _{10}(X)+b$ for $X=L_{\mathrm{X}}, M_{500}$. The raw scatter in the data, $\sigma_{\text {raw }}$, is computed for each fitting method.

\begin{tabular}{|c|c|c|c|}
\hline Method & $a$ & $b$ & $\sigma_{\text {raw }}$ \\
\hline \multicolumn{4}{|c|}{$P_{1.4}-L_{\mathrm{X}}$, excluding CL $0217+70$} \\
\hline $\operatorname{BCES}(\mathrm{X} \mid \mathrm{Y})$ & $2.61 \pm 0.27$ & $-92.60 \pm 11.91$ & 0.50 \\
\hline $\operatorname{BCES}(\mathrm{Y} \mid \mathrm{X})$ & $1.45 \pm 0.16$ & $-40.82 \pm 7.24$ & 0.36 \\
\hline Bisector & $1.90 \pm 0.11$ & $-60.86 \pm 5.13$ & 0.38 \\
\hline Orthogonal & $2.34 \pm 0.21$ & $-80.84 \pm 9.33$ & 0.45 \\
\hline \multicolumn{4}{|c|}{$P_{1.4}-L_{\mathrm{X}}$, including CL $0217+70$} \\
\hline $\operatorname{BCES}(\mathrm{X} \mid \mathrm{Y})$ & $2.47 \pm 0.26$ & $-86.68 \pm 11.78$ & 0.53 \\
\hline $\operatorname{BCES}(\mathrm{Y} \mid \mathrm{X})$ & $1.32 \pm 0.19$ & $-34.72 \pm 8.38$ & 0.39 \\
\hline Bisector & $1.76 \pm 0.16$ & $-54.59 \pm 7.21$ & 0.41 \\
\hline Orthogonal & $2.17 \pm 0.24$ & $-73.23 \pm 10.60$ & 0.47 \\
\hline \multicolumn{4}{|c|}{$P_{1.4}-M_{500}$} \\
\hline $\operatorname{BCES}(\mathrm{X} \mid \mathrm{Y})$ & $5.31 \pm 0.77$ & $-54.55 \pm 11.42$ & 0.61 \\
\hline $\operatorname{BCES}(\mathrm{Y} \mid \mathrm{X})$ & $2.89 \pm 0.28$ & $-18.61 \pm 4.17$ & 0.44 \\
\hline Bisector & $3.77 \pm 0.30$ & $-31.60 \pm 4.38$ & 0.47 \\
\hline Orthogonal & $5.16 \pm 0.74$ & $-52.29 \pm 10.99$ & 0.59 \\
\hline
\end{tabular}

$M_{500}$ relation. Fig. ii.33 shows the BCES orthogonal fit along with the equivalent BCES orthogonal fit by Cassano et al. (2013). We use a total of 64 haloes in this analysis, and Cassano et al. (2013) use 25. Despite this difference in sample size, we see identical best-fitting parameters for the BCES orthogonal method.

We use Fig. ii.34 as an illustration as to the importance of homogeneous measurements. Fig. ii.34 shows clusters that have masses measured via both the SZ effect and through their X-ray luminosity from the MCXC and PSZ1 catalogue. The MCXC (Piffaretti et al., 2011) estimates $M_{500}$ use the X-ray luminosity of the cluster as a proxy, extrapolating from relations between the two. Similarly, PSZ1 (Planck Collaboration et al., 2015a) estimates mass using the Compton $Y_{\text {SZ }}$ parameter. This illustrates clearly both a systematic discrepancy as well as random error between the types of measurements. The $M_{\mathrm{X}, 500}$ measurements are systematically higher than the $M_{\mathrm{YZ}, 500}$ measurements. There is also large discrepancies between measurements at large redshift, though this is to be expected as objects farther away are more difficult to measure accurately.

\section{§ii.2.4. The detection rate of diffuse cluster emission within the EoR0 field}

Here we examine the detection rate of radio haloes, relics, and phoenices within the EoR0 field and compare these to clusters in which no such emission is seen. For these comparisons we consider only those clusters within the MCXC and PSZ1 


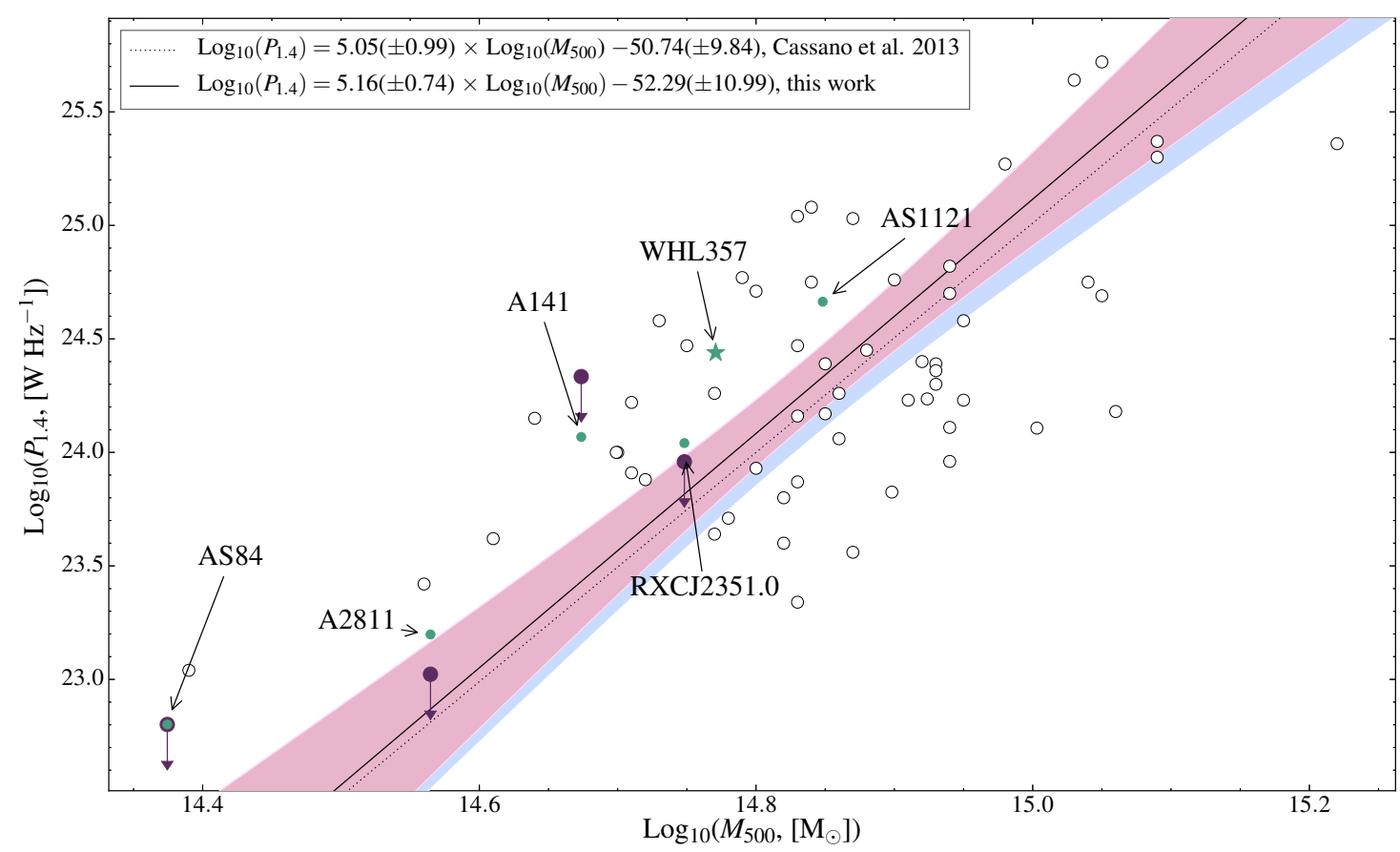

Figure ii.33: The $P_{1.4}-M_{500}$ scaling relation updated with the new halo detections in this paper. The fits are from this work (solid black line with mauve confidence interval) and Cassano et al. (2013; dotted black line with powder-blue confidence interval). The points are as in Fig. ii.32 except the green star indicates a halo with an exactly calculated spectral index. Note that, as with Fig. ii.32 radio power is computed assuming a flat $\Lambda \mathrm{CDM}$ cosmology, with $H_{0}=70 \mathrm{~km} \mathrm{~s}^{-1} \mathrm{Mpc}^{-1}, \Omega_{m}=0.3$, and $\Omega_{\Lambda}=0.7$, as compared to the rest of $\S$ ii which uses $H_{0}=68 \mathrm{~km} \mathrm{~s}^{-1} \mathrm{Mpc}^{-1}$.

catalogue, as a majority of ACO clusters do not have available masses, and a significant portion do not have redshifts. Fig. ii.35 shows cluster mass against redshift. Up until recently it has only been possible to detect radio haloes and relics in nearby clusters, except in the most massive and luminous examples (e.g., Abell 2744). This plot effectively demonstrates the power of the MWA to detect diffuse cluster emission in the redshift range 0.02-0.5, with uniformly distributed masses and redshifts over this range from catalogue-selected clusters. Fig. ii.35 also shows that with such sensitivity we are now probing the diffuse emission in nearby X-ray-emitting low-mass clusters.

The two major limiting factors in the detection of such emission are the resolution and sensitivity of the telescope. The MWA as a low-frequency telescope is limited in its resolution by the maximum baseline at $2873.3 \mathrm{~m}$. The EoR0 field in particular has a beam size of $\sim 2.3$ arcmin, which, when considering only resolution, means that the viable detection range for distant haloes is $\operatorname{LLS}_{500-1000 \mathrm{kpc}}(z) \leq$ 0.22-0.67. Beyond these redshifts, any potential haloes if detected become point sources as they appear the same angular size as the synthesized beam. The second issue is sensitivity; the EoR0 field reaches a sensitivity of approximately 2.3 mJy beam ${ }^{-1}$ in the best regions of the image. The lowest theoretical sensitivity of the Phase I MWA is approximately $1.7 \mathrm{mJy}^{-1}$ beam $^{-1}$ (Franzen et al., 2016). 
Table ii.6: Clusters with diffuse emission and available mass measurements included in Fig. ii.35.

\begin{tabular}{lccc}
\hline \hline Cluster & $\begin{array}{c}M_{500} \\
\left(\times 10^{14} \mathrm{M}_{\odot}\right)\end{array}$ & Type & New \\
\hline Abell 0013 & $2.79^{a}$ & $\mathrm{R}$ & $\times$ \\
Abell 0085 & $4.90^{a}$ & $\mathrm{R}$ & $\times$ \\
Abell 0133 & $3.08^{a}$ & $\mathrm{P}$ & $\times$ \\
Abell 0141 & $4.48^{a}$ & $\mathrm{H}$ & $\checkmark$ \\
Abell 2556 & $2.47^{b}$ & $\mathrm{cP}$ & $\checkmark$ \\
Abell 2721 & $3.77^{a}$ & $\mathrm{cR}$ & $\checkmark$ \\
Abell 2744 & $9.56^{a}$ & $\mathrm{H}, \mathrm{R}$ & $\times$ \\
Abell 2751 & $1.26^{b}$ & $\mathrm{R}$ & $\checkmark$ \\
Abell 2798 & $1.31^{b}$ & $\mathrm{cR}$ & $\checkmark$ \\
Abell 2811 & $3.67^{a}$ & $\mathrm{H}$ & $\checkmark$ \\
Abell 4038 & $2.03^{b}$ & $\mathrm{P}$ & $\times$ \\
Abell S0084 & $2.36^{b}$ & $\mathrm{cH}$ & $\checkmark$ \\
Abell S1121 & $7.05^{a}$ & $\mathrm{H}$ & $\checkmark$ \\
RXC J2351.0-1934 & $5.60^{a}$ & $\mathrm{cH}, \mathrm{cR}, \mathrm{cR}$ & $\checkmark$ \\
MACS J2243.3-0935 & $10.07^{a}$ & $\mathrm{H}$ & $\times$ \\
GMBCG J357.91841-08.97978 & $5.90^{a}$ & $\mathrm{cH}$ & $\checkmark$ \\
\hline${ }^{a} M_{\text {YZ,500 (Planck Collaboration et al., } 2015 a)}$ & & \\
${ }^{a} M_{\mathrm{X}, 500}$ (Piffaretti et al., 2011), truncated to two decimal places.
\end{tabular}




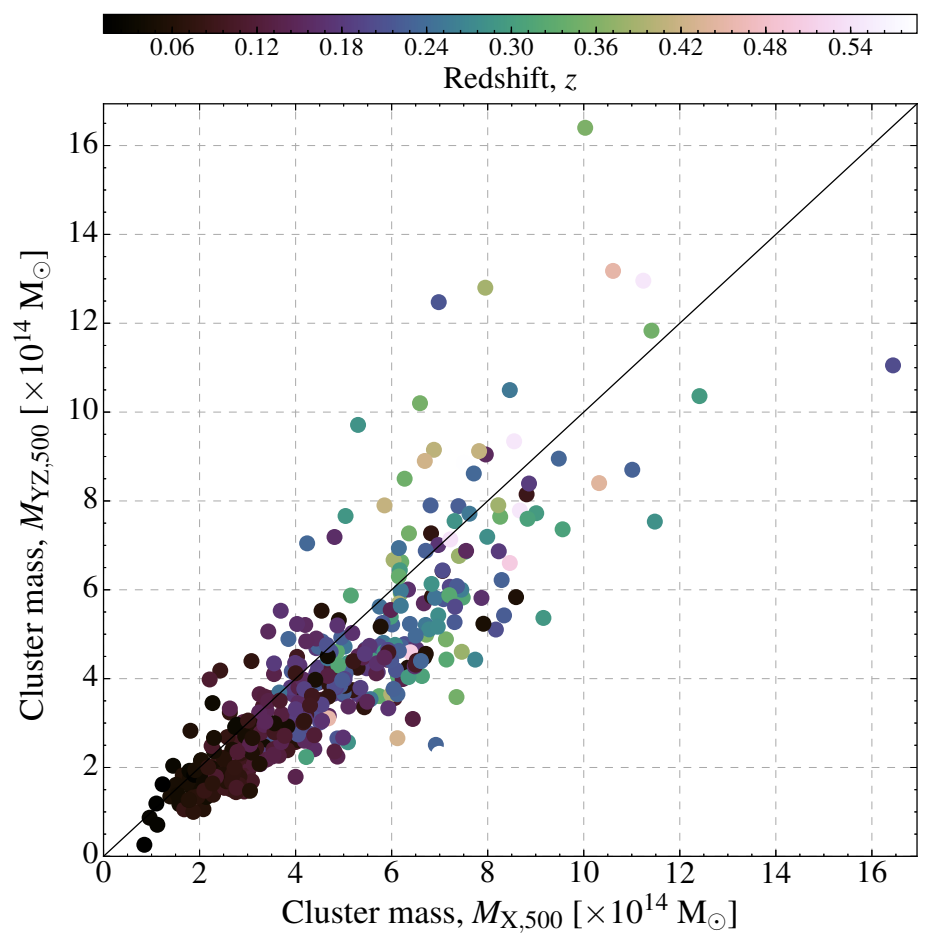

Figure ii.34: The difference between $M_{\mathrm{X}, 500}(\mathrm{MCXC})$ and $M_{\mathrm{YZ}, 500}$ (PSZ1) measurements. This sample is for all clusters within the MCXC and PSZ1 catalogue that have both measurements. $M_{\mathrm{X}, 500}$ measurements utilise the cluster X-ray emission as a proxy for estimated mass, whereas $M_{\mathrm{YZ}, 500}$ uses the Sunyaev-Zel'dovich (SZ) effect as the mass proxy. The solid black line is simply a line indicating equal mass measurements.

However the sensitivity here is not the limiting factor in detecting high-redshift haloes. For the redshift range $z=0.22-0.67$, a halo the size of the beam could theoretically be detected with $1.4 \mathrm{GHz}$ power in the range of $P_{1.4}(z=0.22-0.67) \geq$ $0.3-2 \times 10^{23} \mathrm{~W} \mathrm{~Hz}^{-1}$. This entire range falls below the what is typically seen of cluster haloes (e.g. Cassano et al., 2013; Kale et al., 2015; Shakouri et al., 2016). The solid, black line in Fig. ii.35 shows the theoretical limit for detecting $1 \mathrm{Mpc}$ radio haloes given the $P_{1.4}-M_{500}$ relation found in §ii.2.3.2 and assuming a spectral in index of -1.3 . We see that haloes detected as part of this work lie above this limit, as expected, but note that relics are not bound by the same limit.

The somewhat uniform distribution of detections shown Fig. ii.35 is not surprising given the approximate limits above, and with the sensitivity and resolution of the EoR0 field, the entire redshift space covered by the Planck and MCXC catalogues are available for halo detection.

\section{§ii.3. Conclusions about the EoR0 field}

In this section we have presented diffuse cluster emission seen within the EoR0 field. We have presented 11 haloes, 9 relics, and 3 phoenices, of which 9 are new 


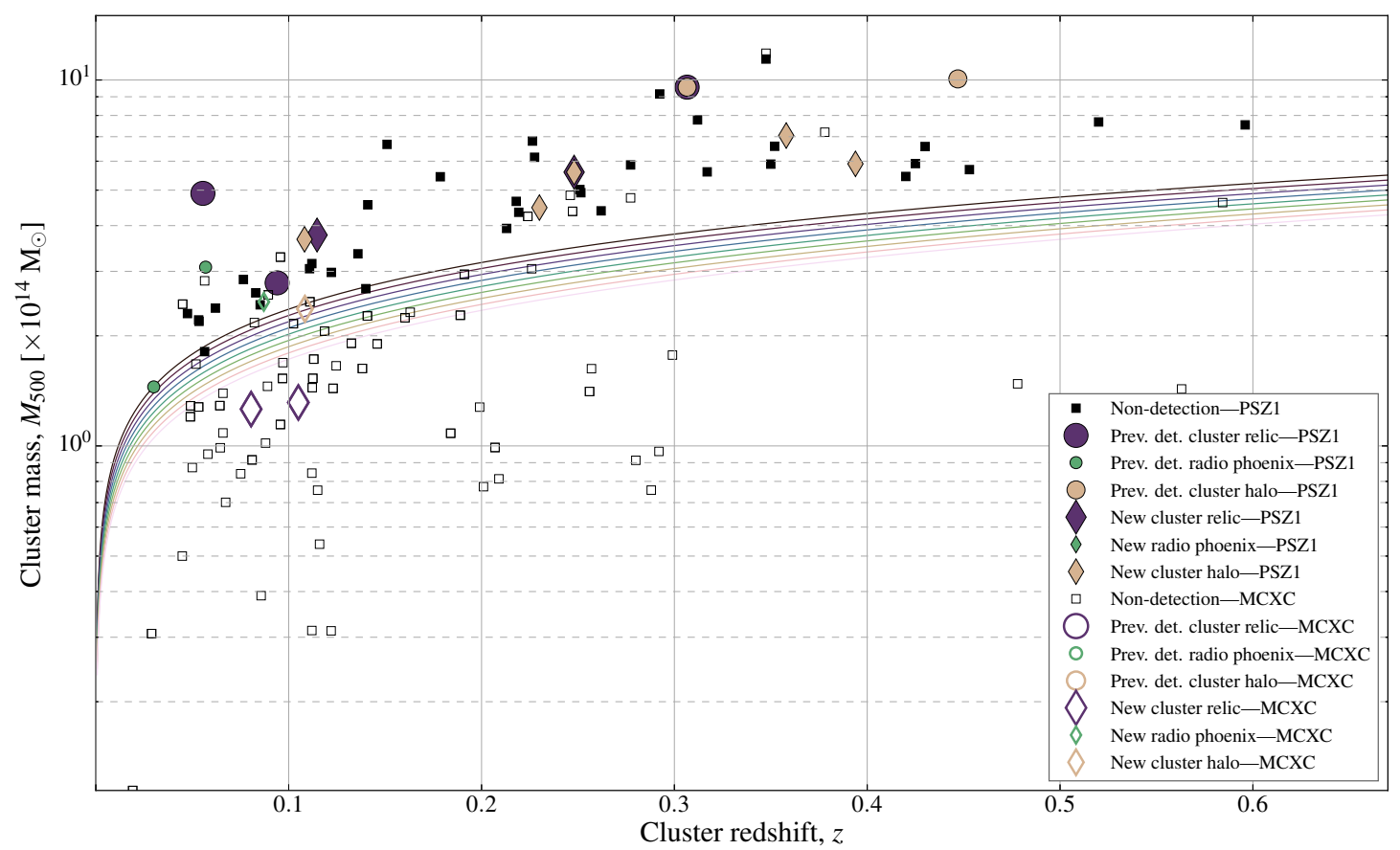

Figure ii.35: Cluster mass against redshift for clusters within the MCXC and PSZ1 catalogues. The filled points are those using the PSZ1 $M_{\mathrm{YZ}, 500}$ measurements and unfilled points are those using the MCXC $M_{500}$ measurements. Where clusters appear in both catalogues we use the PSZ1 $M_{\mathrm{YZ}, 500}$ measurements. The coloured lines indicate the limit at which a $1 \mathrm{Mpc}$ halo can be detected given the $P_{1.4}-M_{500}$ scaling relation in §ii.2.3 assuming a sensitivity of $2.3 \mathrm{mJy}_{\text {beam }}{ }^{-1}$, beam size of 2.3 arcmin, and spectral indices $\{-1.1,-1.2,-1.3,-1.4,-1.5,-1.7,-1.8\}$, where a lower spectral index requires a lower mass cluster. No haloes are detected below this limit. Table ii.6 provides details of the clusters with detected diffuse emission included here. References for redshifts: Abell et al. (1989), Dalton et al. (1994), Alonso et al. (1999), Batuski et al. (1999), de Propris et al. (1999), Jones and Forman (1999), Struble and Rood (1999), Caretta et al. (2002), Zaritsky et al. (2006), Coziol et al. (2009), Wen et al. (2010), Piffaretti et al. (2011), Williamson et al. (2011), Chon and Böhringer (2012), Mahajan et al. (2012), Hicks et al. (2013), Pearson and Batuski (2013), Wen and Han (2013), Planck Collaboration et al. (2014), Bleem et al. (2015), Liu et al. (2015), and Planck Collaboration et al. (2015b). 
haloes, 6 are new relics, and 1 is a new phoenix, or candidates of each. Along with these, we have presented 6 sources that may be either haloes, relics, phoenices, or relic AGN emission from ancient radio galaxies. Where possible we determine 168 $\mathrm{MHz}$ flux densities, estimate angular and linear sizes, and estimate spectral indices or limits. With these detections we examine the incidence rate of such emission, finding that the MWA is beginning to see emission without bias beyond what is present in the catalogues clusters are drawn from. Further, we update the $P_{1.4}-L_{\mathrm{X}}$ and $P_{1.4}-M_{500}$ scaling relations of radio halo power with cluster X-ray luminosity and mass. We find nearly identical best-fitting parameters to the relations as previous works (i.e. Cassano et al. 2013) despite an increase in sample size. 


\section{§iii. Serendipity and follow-up observations}

The primary goal of this work has been the search for diffuse cluster emission using MWA images in the form of the EoRO field and GLEAM. During the course of this, interesting radio emission not considered a halo or relic has been found and examined. This section presents two such objects, as well as a follow-up of a tentative halo. First, a dying radio galaxy at the centre of the cluster Abell S1136, then diffuse emission resembling a cluster halo within Abell S1063, and finally a possible spiral galaxy hosting a radio-loud AGN with disturbed, doublelobed structure.

\section{§iii.1. Diffuse emission within Abell S1136}

\section{§iii.1.1. A dying radio galaxy associated with ESO 470-G020}

The AGN of a radio galaxy has a typical lifetime on the order of $\sim 10^{8} \mathrm{yr}$ (Cordey, 1986) and so there will be a stage in its lifetime where the core does not produce new electrons to power the jets. However, the radio plasma that makes up the lobes does not instantaneously vanish. The rate of energy loss of the electrons is proportional to $-E^{2}$, and so the highest-energy electrons lose energy more quickly. The result is the electron population of the old radio plasma has comparatively more low-energy electrons, giving the steep-spectrum often observed (Komissarov and Gubanov, 1994). Such dying sources are seldom seen, which is a result of both a lack of sensitivity with radio instruments as well as the short lifetime of this dying phase (Cordey, 1986; Giovannini et al., 1988). The first such detection was made by Cordey (1987) with a radio source, B2 0924+30, showing the expected characteristics of a dying radio galaxy associated with IC 2476. Currently, there are still only a small number of confirmed dying radio galaxies, and larger-scale searches have been performed with the hope of detecting more (e.g. Murgia et al., 2011).

Presented in §ii.2.2.23 was the diffuse emission associated with the cluster Abell S1136. We present the cluster once more in Fig. iii.1. The nature of this emission was not understood and the morphology of the source did not appear to represent either a relic or halo. Thus, we considered that the emission may be that of a dying radio galaxy - the old lobes of the central elliptical ESO 470-G020. It is common for BCGs and cD galaxies to host radio lobes, and perhaps ESO 470-G020 was no different. However, the lack of emission at $1.4 \mathrm{GHz}$ suggests a steep spectral index, and since no core is detectable in the EoR0 field we suggest that, if a radio galaxy, it is not in a stage of normal activity. 


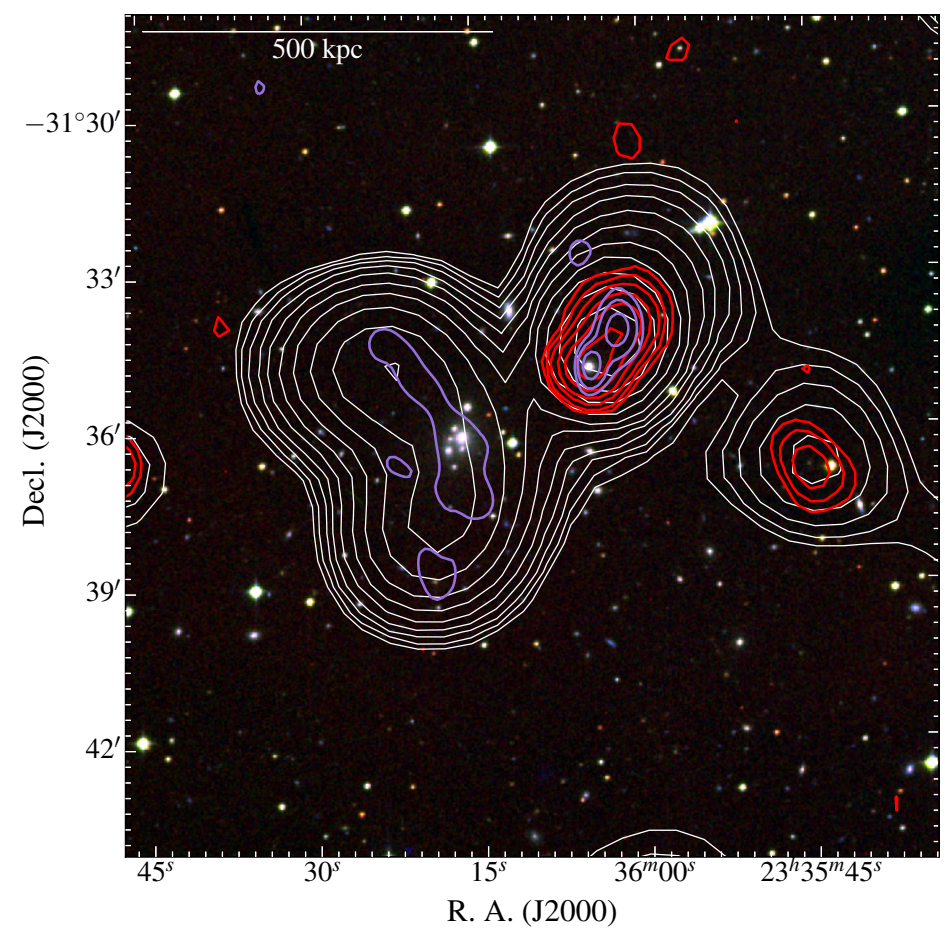

Figure iii.1: The centre of Abell S1136 showing elongated diffuse emission, as presented in §ii.2.2.23. The background is an RGB image with contours overlaid as follows: EoR0, white, beginning at $10 \mathrm{mJy}^{\text {beam }}{ }^{-1}$ increasing by a factor of $\sqrt{2}$; NVSS, red, beginning at $1.5 \mathrm{mJy}_{\text {beam }}{ }^{-1}$ increasing by a factor of 2 ; TGSS, medium purple, beginning at $14.4 \mathrm{mJy}_{\text {beam }}{ }^{-1}$ also increasing by a factor of 2 . 
Table iii.1: The measured flux densities for the diffuse source across the narrow GLEAM bands. The central frequency, $\nu_{c}$, is that given within the FITS header of the GLEAM images, and the local rms noise, $\sigma_{\mathrm{rms}}$, is that calculated by BANE.

\begin{tabular}{cccc}
\hline \hline Band & $\begin{array}{c}\nu_{\mathrm{c}} \\
(\mathrm{MHz})\end{array}$ & $\begin{array}{c}S_{\nu} \\
(\mathrm{mJy})\end{array}$ & $\begin{array}{c}\sigma_{\text {rms }} \\
\left(\mathrm{mJy} \mathrm{beam}^{-1}\right)\end{array}$ \\
\hline $118-126$ & 122.2 & $985 \pm 108$ & 34.0 \\
$126-134$ & 129.9 & $789 \pm 96$ & 35.0 \\
$139-147$ & 142.7 & $697 \pm 77$ & 22.8 \\
$147-154$ & 150.4 & $613 \pm 88$ & 21.5 \\
$154-162$ & 158.1 & $524 \pm 71$ & 18.4 \\
$162-170$ & 165.8 & $506 \pm 70$ & 17.0 \\
$170-177$ & 173.4 & $548 \pm 84$ & 19.1 \\
$177-185$ & 181.1 & $454 \pm 74$ & 17.5 \\
$185-193$ & 188.8 & $428 \pm 68$ & 15.4 \\
$193-200$ & 196.5 & $342 \pm 61$ & 16.7 \\
$200-208$ & 204.2 & $371 \pm 69$ & 17.8 \\
$216-223$ & 219.5 & $367 \pm 85$ & 16.5 \\
$223-231$ & 227.2 & $318 \pm 94$ & 16.9 \\
\hline
\end{tabular}

\section{§iii.1.2. Follow-up analysis with GLEAM}

As the emission within Abell S1136 is detected in all 20 GLEAM narrow bands, we make use of these to measure the spectral index of the entire diffuse region. As seen in Fig. ii.26, the diffuse emission at $168 \mathrm{MHz}$ at the resolution of the MWA blends with the nearby double source, NVSS J233603-313431. Despite this, we make use of AEGEAN to attempt to de-blend the diffuse region and NVSS J233603-313431. This is successful for a majority of the bands, especially at the higher frequencies. However, due to the lower resolution of the low-frequency bands we are unable to disentangle the sources with AEGEAN. Table iii.1 shows the flux densities within each frequency band for the diffuse region. Bands not present are those that had the double and diffuse source fit as a single source, or those that decomposed the emission into three components, the third blending between the diffuse region and NVSS J233603-313431.

Fig. iii.2 shows the SED of the diffuse region within Abell S1136. The spectral index across the GLEAM bands is calculated to be $\alpha=-1.81 \pm 0.12$ which has the steepness expected from the lack of detectable $1.4 \mathrm{GHz}$ or $843 \mathrm{MHz}$ emission from NVSS and SUMSS. The SED here is assuming that contributions from point sources are negligible. This is an appropriate assumption given the lack of detection of point sources in the NVSS or SUMSS within the emission which suggests that any point sources, if present, must not contribute significantly to any flux. Any discrepancy due to embedded sources would be superseded by the uncertainty in the flux density measurements and the following spectral index calculation. 


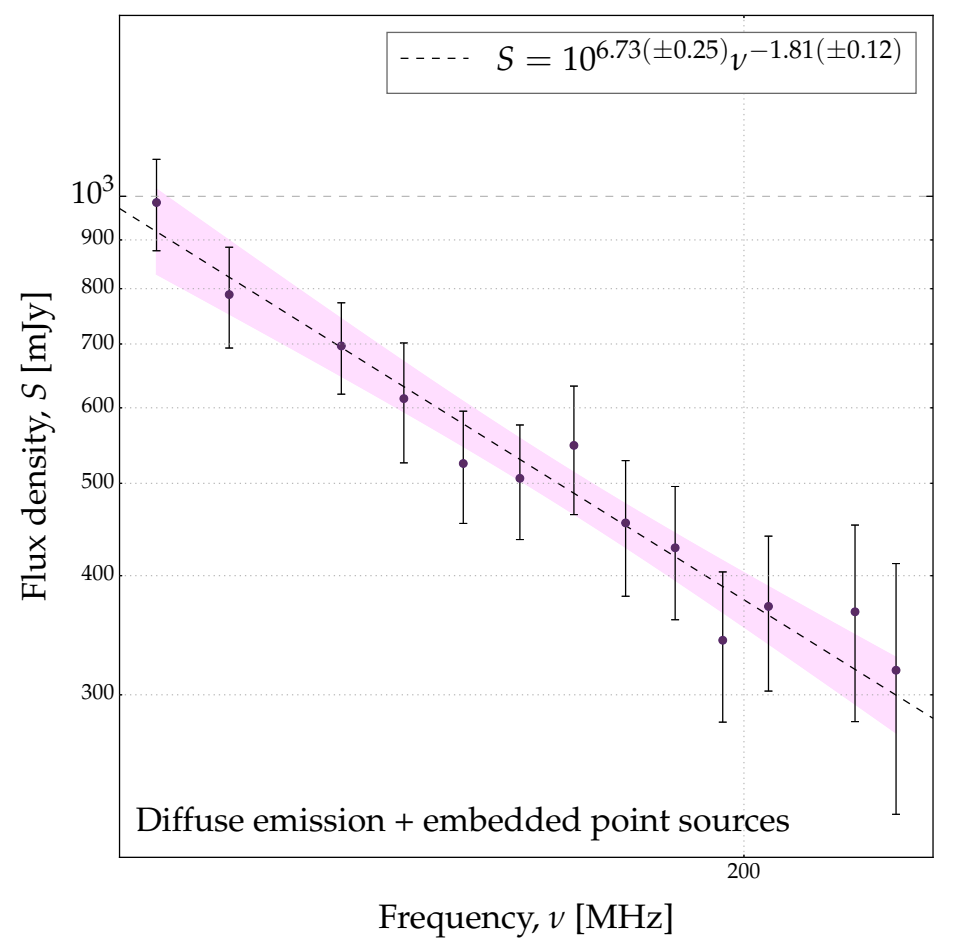

Figure iii.2: The SED of the diffuse emission within Abell S1136. This does not take into account any embedded point sources, which will be discussed in §iii.1.3. The shaded region represents a 95 per cent confidence interval to the fit as in Fig. ii.32.

\section{§iii.1.3. Follow-up observations of Abell S1136 at $2.1 \mathrm{GHz}$}

On April 30, 2016, director's discretionary time (DDT) was used to make an observation of the cluster Abell S1136 with the ATCA in the 6A configuration with the $16 \mathrm{~cm}$ band. We are able to get $\sim 308$ minutes of integration time on the diffuse source, though this is not enough time to allow rotation of the Earth to maximally fill the $u v$ plane. The $6 \mathrm{~A}$ configuration has a largest baseline of 5938.8 $\mathrm{m}$ between the W4 and W392 stations. This large separation allows observations to view the compact cores of radio galaxies. Conversely, the shortest baseline is 627.6 $\mathrm{m}$ between the W4 and W45 stations and gives sensitivity to somewhat extended structures, up to approximately 1 arcmin. However, with only the $6 \mathrm{~A}$ configuration we miss information on spatial scales sampled by the other configurations offered by the ATCA, especially missing out on the 30 metre minimum baseline that the ATCA is capable of. Since 2010, the ATCA has been operating with the Compact Array Broad-band Backend (CABB; Wilson et al., 2011) which allows the use of two intermediate frequency (IF) ranges of 2049 channels or $2048 \mathrm{MHz}$ each. For $16 \mathrm{~cm}$ observations these two IFs are set to the same frequency range as it covers 95 per cent of the available bandwidth in that band.

The $16 \mathrm{~cm}$ band is centred on $2100 \mathrm{MHz}$ and has an effective bandwidth of 1100-3100 MHz. This band gives the closest frequency to the EoR0 field of 168 $\mathrm{MHz}$, but still offers a higher frequency than even the NVSS data, which can allow the exploration of the spectral index of, at the very least, any faint point 


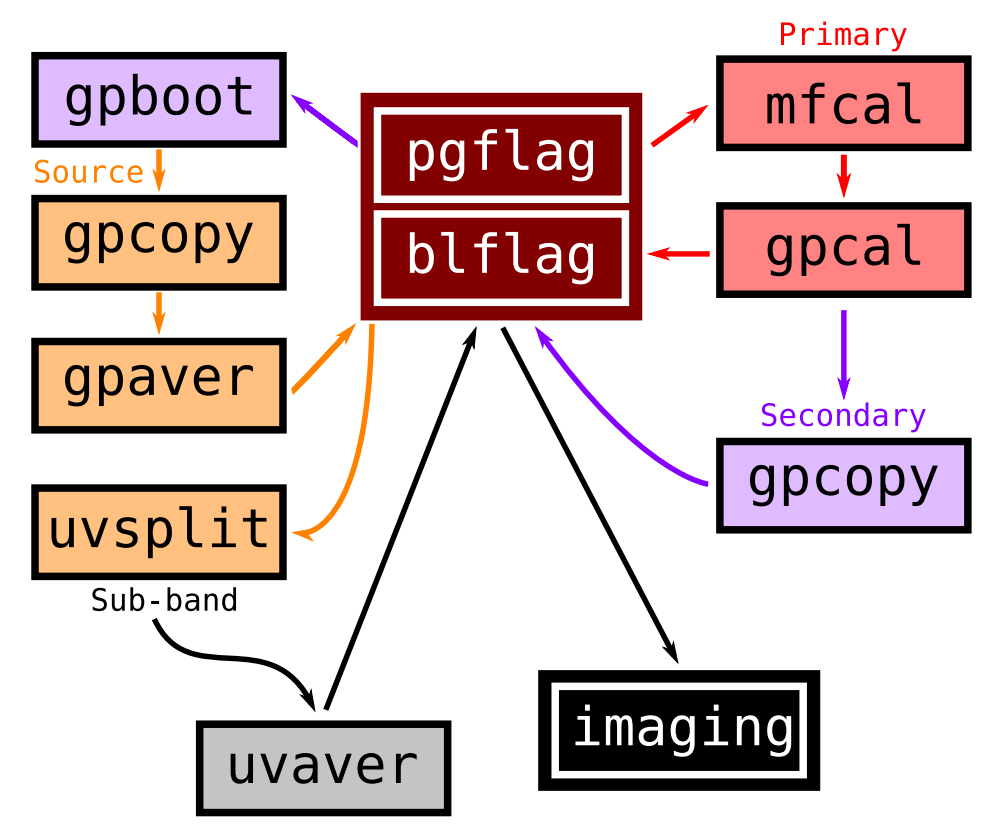

Figure iii.3: The RFI flagging and calibration strategy for the initial four sub-bands of the primary and secondary calibrators and the source. At UVSPLIT each initial sub-band is further split into two sub-bands of $214 \mathrm{MHz}$ each.

sources within the diffuse emission seen in Abell S1136 and in particular the core. Despite the ever-increasing radio frequency interference (RFI) in the $16 \mathrm{~cm}$ band, observations were made without major RFI problems, with the exception of at least one 1000 pseudo-Jy spike of RFI during one of the pointings towards Abell S1136. Beyond that, the RFI contaminating the observations was expected within the $16 \mathrm{~cm}$ band.

\section{§iii.1.3.1. Observing and calibrating}

For most radio observations of astronomical sources, at least two calibration sources are required. These sources help to calibrate the amplitude of the flux density of the telescope response, the bandpass of the telescope, the polarisation leakages from the instrument, and the phase. For observations at $16 \mathrm{~cm}$ with the ATCA, the calibrator PKS B1934-638 is suitable for amplitude and bandpass calibration presuming it is up at the time of observation. At 1.4 GHz, PKS B1938-638 has a flux density of $14.9 \mathrm{Jy}$ (Reynolds, 1994). The secondary calibrator, usually responsible for phase calibration, can be any source that does not require a large slew time of the telescope, that is reasonably strong, and that does not show extended structure at the observation frequency. For the observation of Abell S1136, the quasi-stellar source PKS B2313-340 was chosen as the secondary calibrator as it fit the aforementioned requirements. During the observation, the primary calibrator, PKS B1934-638 is visited first to calibrate the telescope for the observation, and is subsequently visited for a further 20 minutes to get data for calibration during data-processing. The secondary, PKS B2314-340, is observed in 2 minute intervals, for each 20 minute observation of the actual source, Abell S1136. The revisiting 
of the secondary every 20 minutes allows the calibration of the phase throughout the observation.

After the observations, the data, including visibilities of the primary and secondary calibrators, and the source itself, are exported in the RPFITS format written by the correlator of the telescope. The reduction of these data are performed almost entirely within the MIRIAD data-reduction software (Sault et al., 1995). Task ATLOD acts as input, converting from RPFITS to MIRIAD data; UVFLAG is used here to flag end channels of the 2049 available due to bandpass rolloff from lack of sensitivity on the edges; UVSPLIT is used to split the data into individual datasets for the calibrators and source. In this process only the second spectral window (IF) is used, as IF1 and IF2 are identical, and IF2 - in this case - produced data that were more well-behaved. Following this, the first-stage RFI flagging is done, performed on the entire $2048 \mathrm{MHz}$ band, minus those edge channels that have already been flagged. There is further flagged done to end channels that show significant RFI, and we are finally left with a $1716 \mathrm{MHz}$ band, which is subsequently split into four $429 \mathrm{MHz}$ sub-bands.

The following calibration steps are outlined in Fig. iii.3. To begin, MFCAL corrects the bandpass shape of the flux calibrator by comparing the observed shape with the expected shape. GPCAL follows which calibrates the antenna response, and then we pass through a round of RFI flagging. The flagging is done with both the task PGFLAG, using an implementation of the SUMTHRESHOLD method (Offringa et al., 2012) in a manual and automated fashion, and the task BLFLAG removing contaminating visibilities manually. This sequence is repeated until the visibilities are free of any obvious RFI.

Once the primary calibrator is sufficiently flagged and calibrated, we copy the calibration tables to the secondary with GPCOPY and perform the same RFI flagging strategy. Following this, GPBOOT is used to rescale the flux density. These calibration tables are then copied to the source and antenna gains are averaged using GPAVER. Similar to the calibrators, initial RFI flagging is then done, and the sub-bands are then split in two, returning the final eight sub-bands of 214 MHz. As phase errors were present in preliminary images, we use UVAVER to remove visibilities corresponding to baseline-hourangle pairs that were showing such errors. A final found of RFI flagging is done before we move to the imaging process.

\section{§iii.1.3.2. Imaging the data}

The imaging process follows from the calibration. As the bandwidth is large (originally $2 \mathrm{GHz}$ ) we split this into eight sub-bands of $214 \mathrm{MHz}$. This number of sub-bands is chosen to determine where phase errors might occur over the entire bandwidth more easily. We find that a number of the bands feature errors due to phase issues, which are somewhat fixed by culling visibilities on certain baselinehourangle pairs. Once we were satisfied that the remaining visibilities were free of the most problematic errors - likely from time-dependent RFI - the actual imaging 


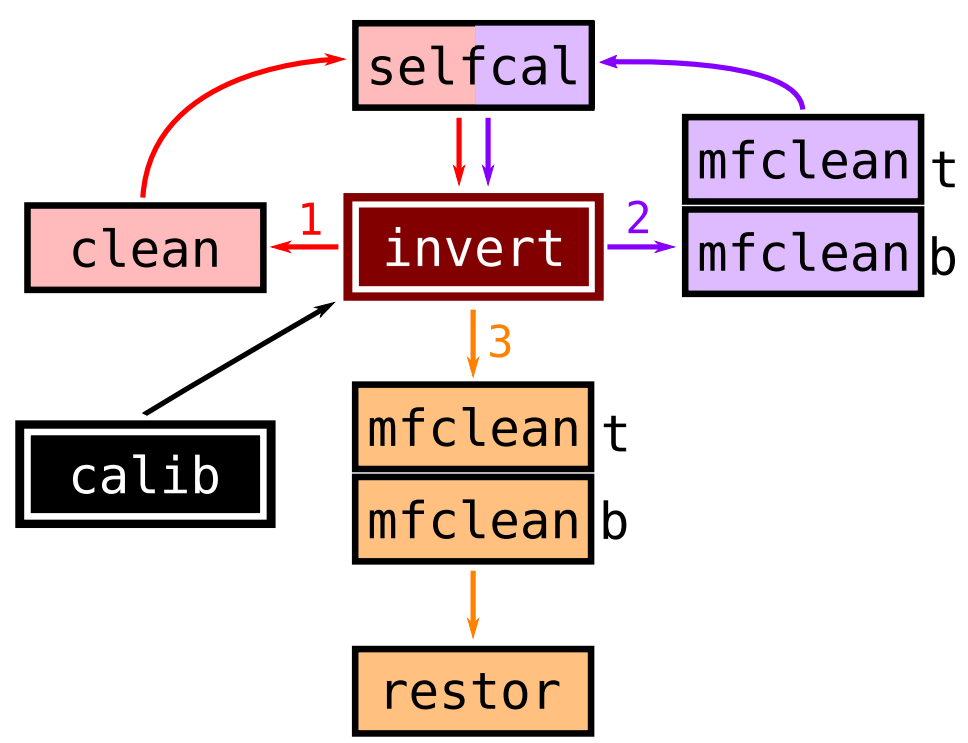

Figure iii.4: ATCA imaging process for the sub-bands of Abell S1136. The two MFCLEAN iterations in each block is for a targeted clean (t) and a blanket clean (b).

begins.

For each final sub-band, the imaging process is outlined in Fig. iii.4. We follow a standard imaging process, first using INVERT to go from visibility space to image space via Fourier transforms. In this instance, we use natural robust 0 weighting in the Briggs weighting scheme in an attempt to enhance any diffuse emission (Briggs, 1995). We follow this with various iterations of deconvolution via cleaning, involving CLEAN and the multi-frequency equivalent, MFCLEAN. These cleaning tasks use an adaptation of the Clark (1980) method, which itself is based on the method of Högbom (1974). The first clean stage is used to get a model to allow for self-calibration with the task SELFCAL, which uses sources within the field above a user-specified clip-level to perform both phase and amplitude calibration. This is performed twice, with the second round using a lower clip level. The second round of cleaning, which involves the second round of self-calibration, using the task MFCLEAN with two iterations. The first uses a targeted clean around the most confusing source in the image. This happens to be a HT galaxy next to our source-of-interest. The extended structure in the HT galaxy's tail is particularly confusing for the clean process, and the brightness of the source overall compounds this. The second iteration of MFCLEAN performs a blanket clean over the inner portion of the image. This effectively cleans around all but the brightest sources. After the second round of self-calibration, we perform one more round of MFCLEAN and finally use the task RESTOR to combine the clean model, synthesized beam, and dirty map produced by the task INVERT to make a real image.

We produced a stacked image of the region centred on $\sim 2.1 \mathrm{GHz}$ by first convolving the images to the lowest band's resolution and using the task LINMOS to perform linear mosiacing. We present this image in Fig. iii.5 and iii.6. To complement this image, we also produce a similar stacked image made from tapered sub-band images. The imaging process for these is simple as it only involves one 
Table iii.2: Sub-band properties for the ATCA observations of Abell S1136. The band is the frequency named by MIRIAD after UVSPLIT and the central frequency, $\nu_{c}$, is the frequency embedded in the FITS file. We use $\nu_{c}$ for calculations. The rms noise is calculated using BANE on cropped images of the central $\sim 1$ degree of the field, and is the rms at the reference pixel of the image.

\begin{tabular}{ccccc}
\hline \hline Image & $\begin{array}{c}\text { Central frequency } \\
(\mathrm{MHz})\end{array}$ & $\begin{array}{c}\text { Restoring beam } \\
\left.\left({ }^{\prime \prime} \times^{\prime \prime}\right)^{\circ}\right)\end{array}$ & $\begin{array}{c}\sigma_{\text {rms }} \\
\left(\mathrm{\mu Jybeam}^{-1}\right)\end{array}$ & $\begin{array}{c}\text { Core flux } \\
(\mathrm{mJy})\end{array}$ \\
\hline 1413 & 1408.5 & $19.31 \times 4.49,-24.3$ & 209.11 & $0.85 \pm 0.21$ \\
1629 & 1672.0 & $16.5 \times 3.78,-24.1$ & 240.96 & $1.00 \pm 0.23$ \\
1845 & 1839.8 & $13.6 \times 3.53,-23.2$ & 170.39 & $0.57 \pm 0.15$ \\
2059 & 2040.5 & $12.08 \times 3.13,-23.2$ & 131.72 & $0.45 \pm 0.12$ \\
2274 & 2274.5 & $10.28 \times 2.61,-22.2$ & 75.16 & $0.51 \pm 0.08$ \\
2488 & 2495.0 & $9.59 \times 2.45,-22.7$ & 69.52 & - \\
2703 & 2726.6 & $10.21 \times 2.46,-27.7$ & 57.91 & - \\
2917 & 2917.6 & $9.33 \times 2.25,-27.8$ & 42.37 & - \\
Stacked & 2114.0 & $19.31 \times 4.49,-24.3$ & 116.67 & - \\
Stacked $^{a}$ & 2097.9 & $77.07 \times 20.25,-51.5$ & 5000 & - \\
\hline
\end{tabular}

${ }^{a}$ Stacked after tapering sub-bands with a 30 arcmin Gaussian.

round of CLEAN. Tapering is done during the inversion from visibility space, where we apply a Gaussian taper of 30 arcmin by 30 arcmin when performing the Fast Fourier Transform (FFT) within the task INVERT. This tapering is done in an attempt to bring out any low-surface-brightness diffuse emission that is seen at low frequencies, where the diffuse emission may be seen for the same reason that more emission is seen within the EoR0 field image than in TGSS - high resolution images can resolve out diffuse flux from a radio map.

\section{§iii.1.4. Results with the ATCA}

After the imaging process, we are left with 8 sub-bands between 1.1 and $3.1 \mathrm{GHz}$. Table iii.2 summarises the sub-band images. In no band is the diffuse emission seen. In the stacked image shown in Fig. iii.6 we do not see even a hint of the emission, however it is clear that there are a number of intruding point sources. One of the motivations for the observation with the ATCA was to determine the spectral index of the proposed core of the emission, ESO 470-G020. We see the core above $4 \sigma_{\text {rms }}$ in 5 of the 8 sub-bands, and measure the integrated flux densities using the in-house PYTHON code. Fig. iii.7 shows the SED within these sub-bands for the core, and the spectral index is calculated to be $\alpha=-1.39 \pm 0.55$. The data are not well behaved and this is likely due to a combination of poor signal-tonoise for the core and perhaps subtle side-lobe artefacts from the nearby HT. We note that further source measuring in this region will produce similar results, and since the diffuse emission is so much brighter than these point sources attempting to subtract the three interloping sources would not increase the accuracy of the 


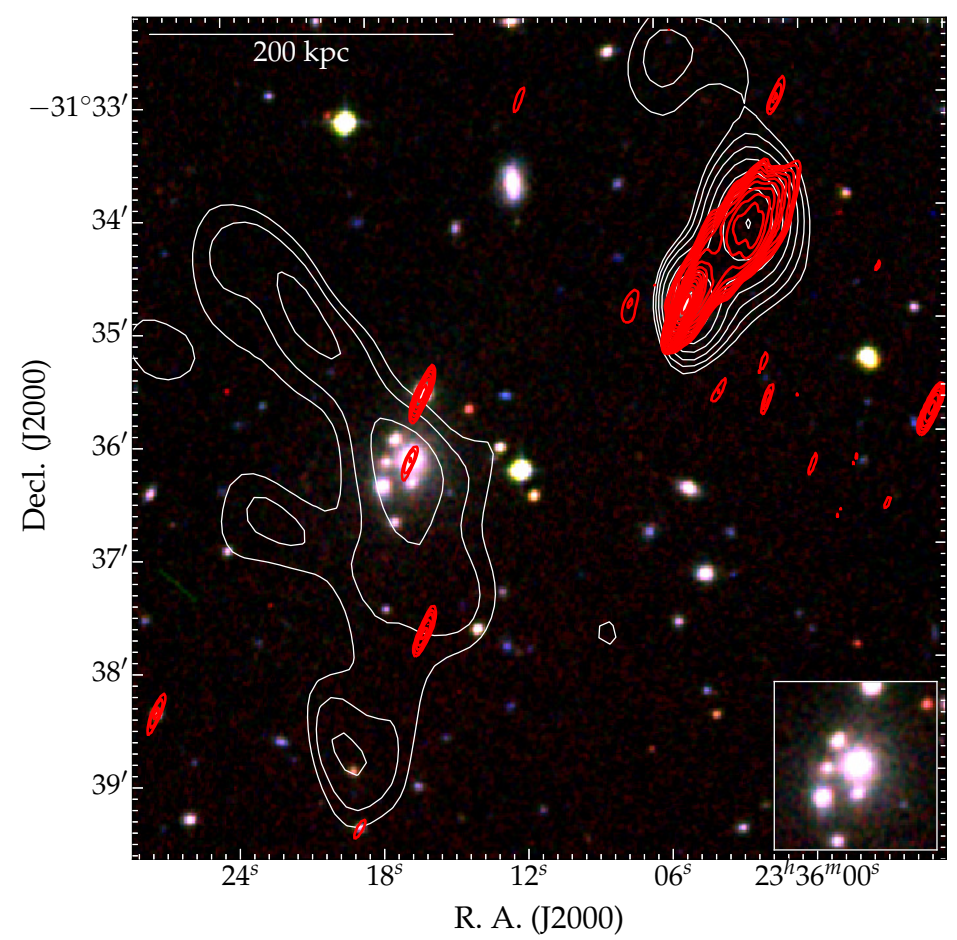

Figure iii.5: The centre of Abell S1136. The background is an RGB image using the IR, red, and blue bands of the DSS2, with contours overlaid as follows: TGSS, white, beginning at $10 \mathrm{mJy}_{\text {beam }}^{-1}$; ATCA $2.1 \mathrm{GHz}$, red, beginning at $0.35 \mathrm{mJy}^{\text {beam }}{ }^{-1}$, both sets increasing with factors of $\sqrt{2}$. Note the spurious sources to the northeast and southwest of the HT galaxy - these are artefacts from the imaging process as the HT is a particularly confusing source for the clean process. The linear scale is at the redshift of the cluster. The inset in the bottom right corner is of the cluster centre. The ATCA stacked image clearly detects the core of the emission and shows its association with ESO 470-G020.

current spectral index measurement within the GLEAM bands, and would require assumptions on the SEDs of each of the three point sources and the diffuse emission itself.

Fig. iii.6 shows the stacked and tapered ATCA images of the cluster. The top panel shows the final stacked image with no tapering, with a single contour of the EoR0 and TGSS images at $10 \mathrm{mJy}^{-1} \mathrm{beam}^{-1}$ (red and black). The bottom panel shows the tapered, stacked image, with contours showing both the TGSS (black) and the non-tapered, stacked ATCA image (red). There is no hint of diffuse emission in this tapered image, though due to side-lobe noise from the bright HT, this is not particularly surprising as any emission may be lost within this noise.

\section{§iii.1.5. Discussion and conclusion on the nature of the emis- sion within Abell S1136}

The emission as seen at low radio frequencies and resolutions (e.g. across the GLEAM bands and within the EoR0 field) is suggestive of some sort of relic AGN 

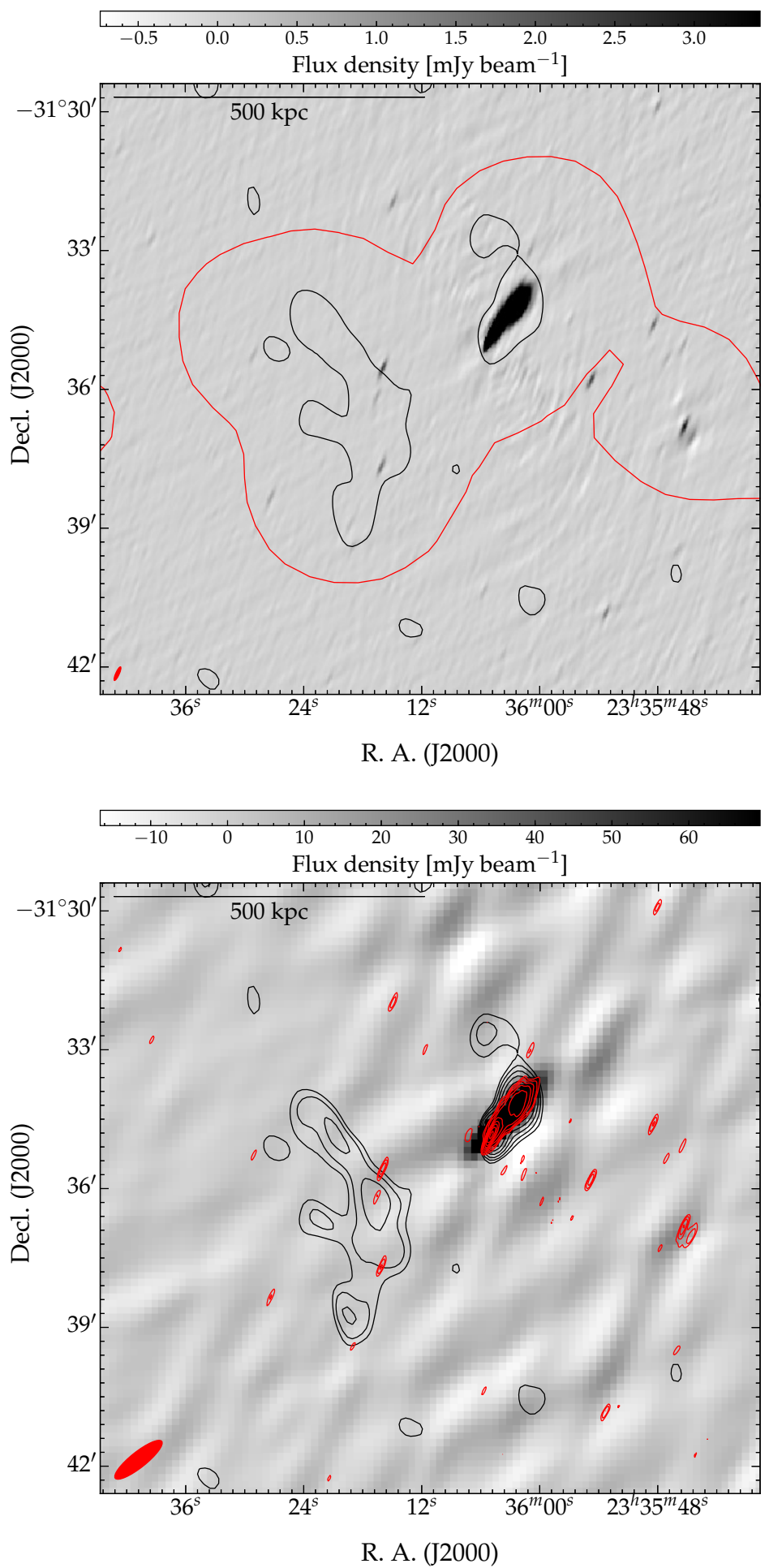

Figure iii.6: Top: The stacked radio image of Abell S1136 centred on $2.1 \mathrm{GHz}$. The single red contour is of the $168 \mathrm{MHz}$ MWA data, and the single black contour is of the $147.5 \mathrm{MHz}$ TGSS data; both contours at $10 \mathrm{mJy}^{\text {beam }}{ }^{-1}$. Bottom: The stacked, tapered radio image, with contours as in Fig. iii.5. The red ellipses in the bottom-left corner of each image are at the size of the synthesized beam of the respective image. The linear scale is at the redshift of the cluster. 


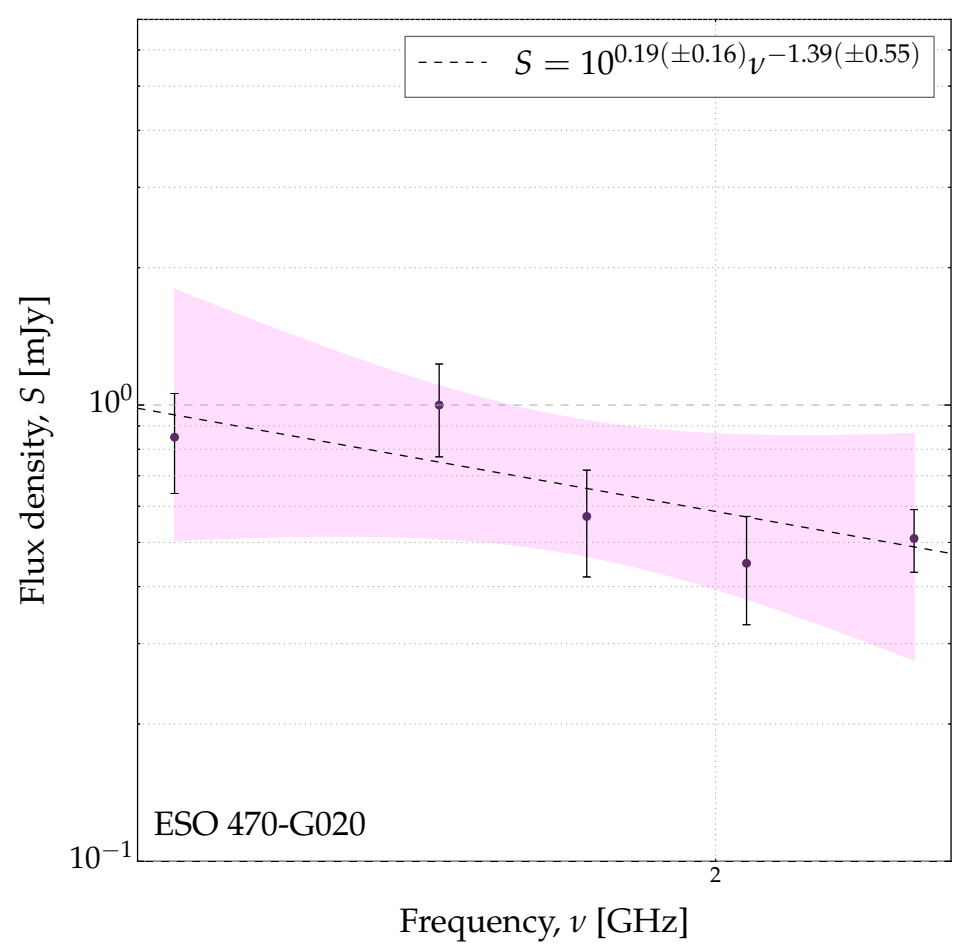

Figure iii.7: The SED of ESO 470-G020 with flux densities from Table iii.2. The core is incredibly faint, and is barely detected in most bands. The three bands not used do not detect the core above $4 \sigma_{\text {rms }}$. The shaded region represents the 95 per cent confidence interval to the fit as in Fig. ii.32.

emission, with tentative lobe-like structure. The TGSS data confirms this, showing a peak at the emission where a possible core galaxy, ESO 470-G020 resides. Other peaks in the TGSS emission do not show optical hosts and are likely the old hotspots of the relic AGN emission. After performing follow-up observations of the cluster with the ATCA in the $16 \mathrm{~cm}$ band, we confirm the presence of a core with weak radio activity and a steep spectral index, likely in its last stages of life. The core is associated with the giant elliptical ESO 470-G020 as expected, and we see another two point sources that are embedded within the diffuse emission nearby, one associated with 2MASX J23361598-3135345 (north with respect to ESO 470-G020) and one previously undetected (south with respect to ESO 470G020). The likeliest scenario for the origin of the emission is from the BCG of the cluster, ESO 470-G020, and relic AGN emission from a central galaxy is consistent with other findings of such dead radio galaxies (e.g. Murgia et al., 2011). 


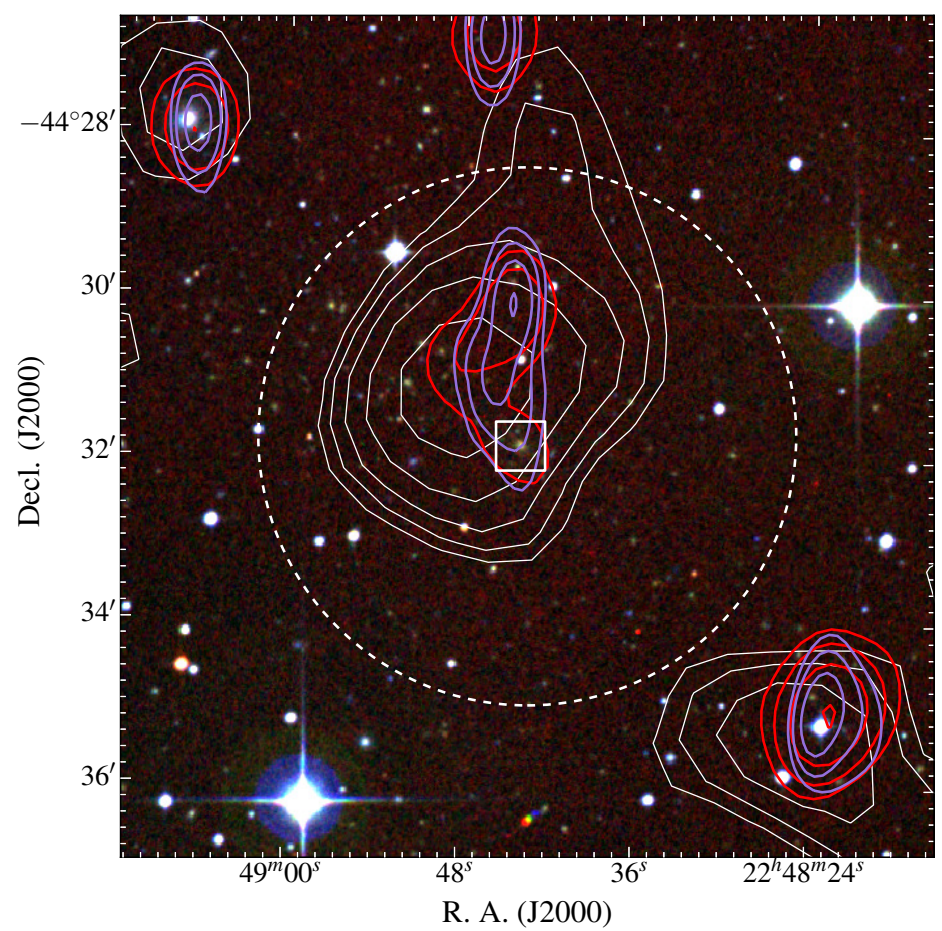

Figure iii.8: The RGB image of Abell S1063 presented in §ii.2.2.20. contours overlaid as follows: EoR0, white, beginning at $50 \mathrm{mJy}^{\text {beam }}{ }^{-1}$ increasing by a factor of $\sqrt{2}$; SUMSS, red, beginning at $5 \mathrm{mJy} \mathrm{beam}^{-1}$ increasing by a factor of 2 ; TGSS, medium purple, beginning at $7.8 \mathrm{mJy}$ beam $^{-1}$ also increasing by a factor of 2 . The dashed circle is centred on the cluster and has a radius of $1 \mathrm{Mpc}$. The white box indicates the BCG of the cluster.

\section{§iii.2. Diffuse emission within Abell S1063}

The nature of the cluster (massive and X-ray luminous) would suggest a halo may be present, and the estimated spectral index of the emission agrees with this hypothesis. However, the morphology of the emission is more reflective of a HT galaxy with a blended point source possibly associated with the BCG, LCRS B224549.3-444744. The cluster has been observed with the ATCA in 2013 with the EW352 configuration (Project code C2837, PI M. Johnston-Hollitt) in a search of diffuse cluster emission from clusters in the South Pole Telescope survey (SPT: Song et al., 2012; Reichardt et al., 2013) though the data had not yielded unambiguous diffuse emission. We use these data in conjunction with observations in the 6A configuration (Project code C2585, PI R. Kale) obtained from the Australia Telescope Online Archive (ATOA) to fully explore the cluster's emission in the frequency range 147.5-3100 MHz. Table iii.3 summarises the properties of the observations used in this work. 
§iii.

Table iii.3: Properties of the ATCA observations of Abell S1063. $\nu_{c}$ is the centre frequency of each observation.

\begin{tabular}{cccccc}
\hline \hline Configuration & Date & $\begin{array}{c}t_{\text {scan }} \\
(\operatorname{mins})\end{array}$ & $\begin{array}{c}\text { Bandwidth } \\
(\mathrm{MHz})\end{array}$ & $\begin{array}{c}\nu_{c} \\
(\mathrm{MHz})\end{array}$ & $\begin{array}{c}\text { Max. scale } \\
(\operatorname{arcmin})\end{array}$ \\
\hline EW352 & $18,20,22-06-2013$ & 224 & 2048 & 2100 & $\sim 21$ \\
6A & $03,04,05-02-2012$ & 543 & 2048 & 2100 & $\sim 1$ \\
\hline
\end{tabular}

\section{§iii.2.1. ATCA data of Abell S1063}

The reduction of ATCA data follows standard procedures of CABB continuum data reduction with MIRIAD outlined in §iii.1.3.

In the data processing, Dr. Qian Zheng first flagged and removed RFI then further flagging the edge channels that are within the bandpass rolloff, as the filters on the correlator have an area of approximately $32 \mathrm{MHz}$ on each side with lessened sensitivity. PKS B1934-638 was used as the bandpass and flux calibrator for both configurations, PKS B2326-477 was the phase calibrator for the EW352 configuration and MRC 2117-614 was the phase calibrator for the 6A configuration. The dataset was split into four sub-bands of $512 \mathrm{MHz}$ width, approximately centred at 1332, 1844, 2356, and $2868 \mathrm{MHz}$.

At this point the data are passed on to the author of this work, and a similar imaging process is followed as outlined in §iii.1.3 (see Fig. iii.4). In the Briggs robust weighting scheme, natural weighting 0 is used in an attempt to enhance any low-surface-brightness, diffuse emission. The multi-frequency clean task MFCLEAN is used for deconvolution using the Clark method. Each sub-band goes through two passes of amplitude and phase self-calibration using the task SELFCAL. The first pass utilises a higher clip level of $50 \mathrm{mJy} \mathrm{beam}^{-1}$ for the lower three bands and $10 \mathrm{mJy}$ beam $^{-1}$ for the highest. The second pass uses a lower clip value of 1 mJybeam ${ }^{-1}$. A single pass of MFCLEAN is used between each round of selfcalibration, where each self-calibration and clean stage uses previously generated clean model as a prior. Finally the sub-bands are convolved to the synthesized beam of the lowest frequency band and stacked using the task LinMos. In this case, it is used to increase the signal-to-noise ratio in the final, stacked $2100 \mathrm{MHz}$ image. This stacked image has an rms noise of $21 \mu \mathrm{Jy}_{\text {beam }}{ }^{-1}$ at the centre, increasing out towards the edges of the image. Table iii.4 summarises the sub-band images, on which we perform measurements. Fig. iii.9 shows the stacked sub-bands convolved to the resolution of the $1332 \mathrm{MHz}$ sub-band centred on approximately $2100 \mathrm{MHz}$ (top panel) and the stacked image after tapering with a 60 arcmin Gaussian beam during the FFT process (bottom panel).

\section{§iii.2.2. Analysis of the central cluster region}

The core of the cluster features four radio sources detected in the ATCA images: The BCG of the cluster, LCRS B224549.3-444744 (Obj. A in Fig. iii.9, the 

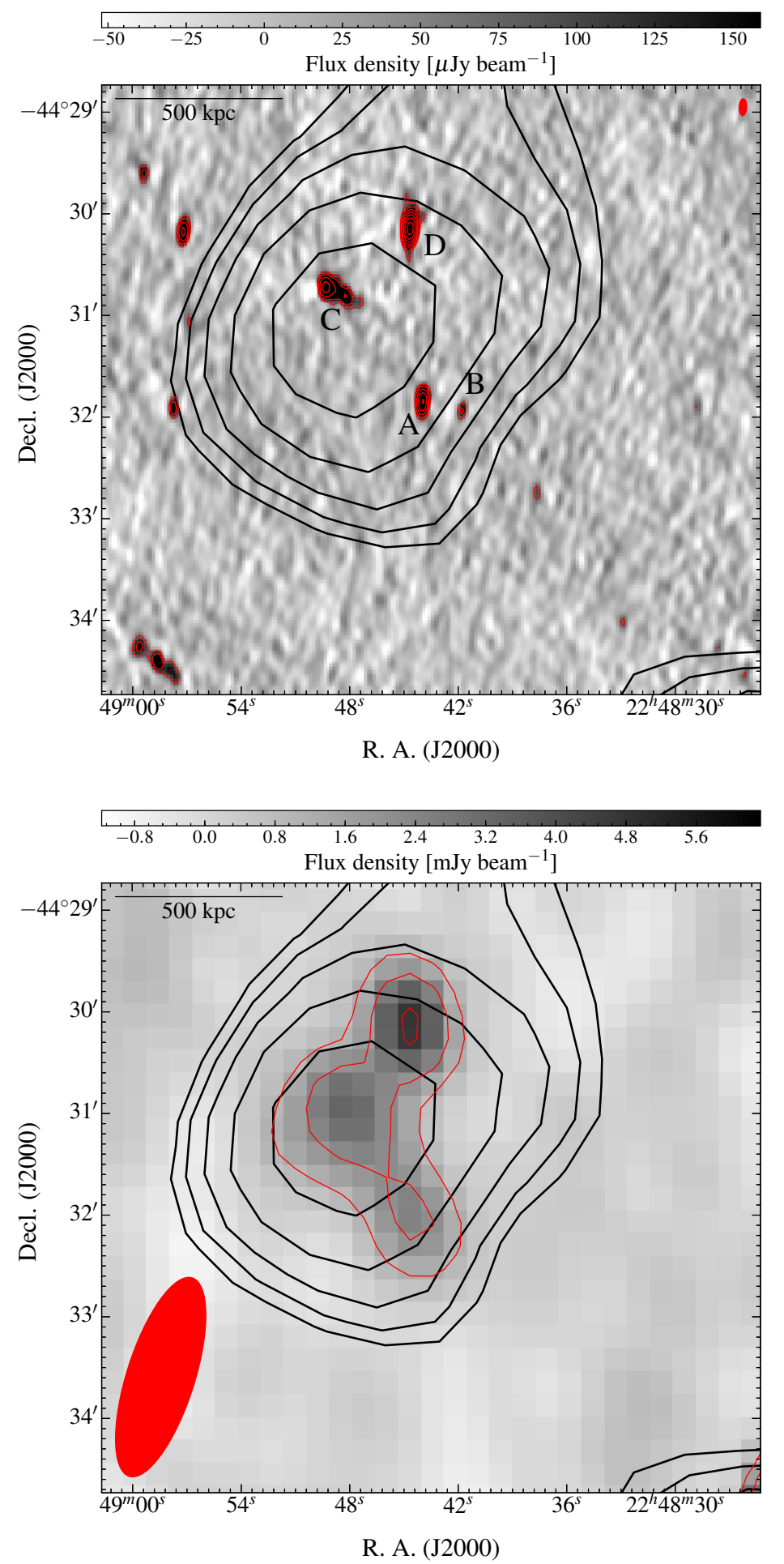

Figure iii.9: Abell S1063. Top: ATCA stacked image of the 1100-3100 MHz band centred on $2100 \mathrm{MHz}$ overlaid with contours as follows: EoR0, black, beginning at 40 $\mathrm{mJy}_{\text {beam }}{ }^{-1}$; ATCA, red, beginning at $72 \mu \mathrm{Jy}_{\text {beam }}{ }^{-1}$. The red ellipse in the topright corner is the size of the synthesized beam. Bottom: Stacked, tapered ATCA image of the same band. The EoR0 contours are as in the bottom panel, with ATCA contours in red, beginning at $1.08 \mathrm{mJy}_{\text {beam }}{ }^{-1}$. The red ellipse in the bottom-left corner is the size of the synthesized beam. ATCA contours in both images are increasing with factors of 2 and the EoR0 contours increase with factors of $\sqrt{2}$. The linear scale is at the cluster's redshift. 
Table iii.4: Sub-band and image properties for the ATCA observations of Abell S1063. The rms noise is calculated using BANE and is for the centre of the image.

\begin{tabular}{cccc}
\hline \hline Image & $\begin{array}{c}\text { Centre frequency } \\
(\mathrm{MHz})\end{array}$ & $\begin{array}{c}\text { Restoring beam } \\
\left({ }^{\prime \prime} \times{ }^{\prime \prime},{ }^{\circ}\right)\end{array}$ & $\begin{array}{c}\sigma_{\text {rms }} \\
\left(\mu \mathrm{Jy} \mathrm{beam}^{-1}\right)\end{array}$ \\
\hline 1332 & 1384 & $9.95 \times 4.45,-3.1$ & 50 \\
1844 & 1873 & $7.57 \times 3.89,1.5$ & 21 \\
2356 & 2349 & $6.10 \times 3.15,0.0$ & 22 \\
2868 & 2811 & $5.13 \times 2.74,-4.0$ & 26 \\
Stacked $^{a}$ & 2034 & $9.95 \times 4.45,-3.1$ & 18 \\
Stacked $^{a}$ & 2251 & $122.9 \times 40.1,-17.7$ & 360 \\
\hline
\end{tabular}

${ }^{a}$ Stacked after tapering sub-bands with a 60 arcmin Gaussian.

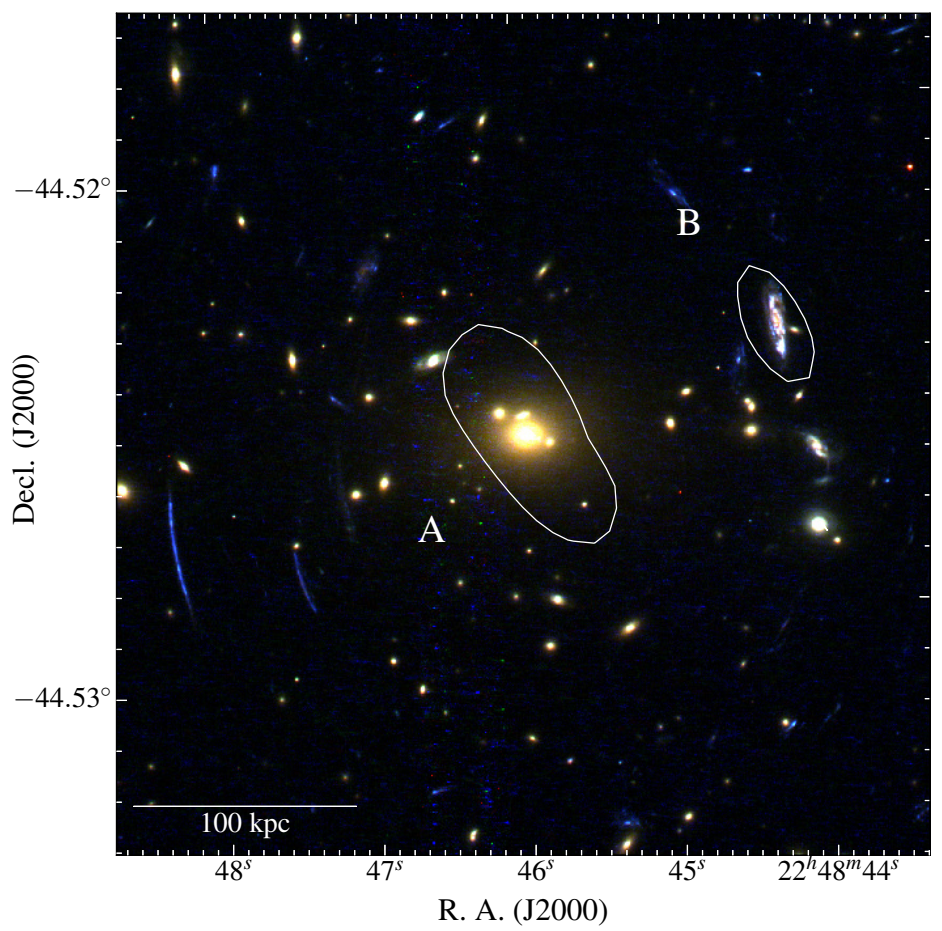

Figure iii.10: The central galaxies of Abell S1063. The background image is a three-colour image made using the HST F814 W, F606 W, and $F 435 \mathrm{~W}$ bands corresponding to red, green, and blue respectively. The HST data is obtained from the Mikulski Archive for Space Telescopes (MAST) from project 14037, PI Lotz. The single white contour is at the $3 \sigma_{\text {rms }}$ level of the stacked ATCA image, and the linear scale is at the redshift of the cluster. The labels are as in the top panel of Fig. iii.9, though note that north is not directly up, which gives a rotation compared to Fig. iii.9. The blue arcs are lensed background galaxies. The spiral (B) shows no lensing in this image. 
central elliptical of Fig. iii.10), is detected in each band, along with the source SUMSS J224845-443025 (Obj. C and D). From the SUMSS and TGSS images it would appear that Obj. D is in fact the core of a HT galaxy, with Obj C its tail. However, upon inspection of the higher resolution ATCA images, we see that the tail is a discrete extended source. This source does not have an optical ID, nor are there sources in NED listed at the peak of the radio emission. The source lies on edge of the X-ray emission from the cluster's centre. For SUMSS J224845-443025 we use AEGEAN to measure the integrated flux density in each sub-band, yielding a spectral index of $\alpha_{1332}^{2868}=-1.11 \pm 0.19$, where flux density measurements have an uncertainty as in Eq. ii.1 but with a 10 per cent term as is typical of the ATCA instead of 5 per cent (plus calibration uncertainty) for the EoR0 field. Fig. iii.10 shows a close-up of the cluster centre, including LCRS B224549.3-444744 and Obj. B, which in this three-colour Hubble Space Telescope (HST) image is shown to be a spiral galaxy with radio emission. This spiral is likely the GALAEXASC source, GALEXASC J224841.77-443157.0, which is considered a lensed background galaxy (Gómez et al., 2012), however this spiral does not show lensing characteristics and likely this association with a lensed galaxy can be attributed to the image being used not having quite the same fidelity as the more recent $H S T$ images.

\section{§iii.2.3. Conclusion on the nature of the emission within Abell S1063}

The high resolution ATCA images confirm that there are embedded point sources within the emission seen at $168 \mathrm{MHz}$ in the EoR0 field. These sources trace the emission seen in SUMSS and the TGSS, though the previous notion that SUMSS J224845-443025 was a HT galaxy is dispelled. While a diffuse cluster halo may be present, it is - with the current data - rendered undetectable. Given that cluster may have recently gone through a merger (Gómez et al., 2012) and its high X-ray luminosity (equivalent to the Bullet Cluster, 1E 0657-56; see e.g. Liang et al., 2000; Markevitch, 2006; Shimwell et al., 2014; Srinivasan, 2015) it was expected that Abell S1063 might host a halo. 


\section{§iii.3. A curious AGN host}

While inspecting the cluster Abell 2685, a radio galaxy with curious morphology, akin to a rose, was observed nearby.

\section{§iii.3.1. Disk galaxies hosting AGN}

Disk galaxies with prominent dust are not typically found to host powerful AGN (see e.g. Abdo et al., 2009), however in rare circumstances have been observed (e.g. the host galaxy of Fornax A, NGC 1316; Dreyer, 1888; Burbidge and Burbidge, 1957). In particular, double-lobed radio sources associated with powerful AGN are seldom seen in such galaxies, and it is thought that the feeble amount of dust in typical ellipticals is what allows the jets to form. Disk galaxies - in particular, spirals - have been seen to produce small jets from a central black hole, classed as Seyfert AGN (Seyfert, 1943; Gallimore et al., 2006; Ulvestad et al., 1981) of various types. This small-scale emission does not typically extended much beyond the optical extent of the host galaxy, and the power is much lower than in normal double-lobed radio galaxies. It is likely the powerful AGN seen are the results of mergers that have created the elliptical galaxies (e.g. Hopkins et al., 2006; Chiaberge and Marconi, 2011). However, there are a small but growing number of spiral galaxies found to host large double-lobed AGN. In this section, we present a curious spiral host of large-scale double-lobed radio emission: ESO 472-G013.

\section{§iii.3.2. The curious host: ESO 472-G013}

The galaxy ESO 472-G013 is unambiguously classified as a spiral (SB: Lauberts 1982; S: Loveday 1996; SBbc: Paturel et al. 2003) in the literature, though this morphology is not inherently clear in the DSS2 images alone. ESO 472-G013 is detected as part of the 6-degree Field Galaxy Survey (6dFGS; Jones et al., 2004) and is reported to have a redshift of $z=0.050718 \pm 0.000150$ (Jones et al., 2009). The galaxy hosts an AGN, with prominent emission from its core as well as a double-lobed structure. The northern lobe appears highly disturbed, which is unusual for a galaxy not part of a galaxy cluster or not within any significantly dense medium. The southern lobe appears much less grandiose in size as well as morphology, though likely this due to projection effects as the southern lobe may be angled away from the observer. Separated by $\sim 112 \operatorname{arcsec}(\sim 111 \mathrm{kpc})$ is ESO $472-$ G012, which is also a spiral galaxy (S: Lauberts 1982; Sbc: Paturel et al. 2003) and has a redshift of $z=0.052193 \pm 0.000053$ (di Nella et al., 1996). ESO 472-G012 shows no peculiar characteristics for a spiral and hosts no detectable radio emission. We mention this companion here due to the close proximity to ESO 472-G013, and discuss in §iii.3.7 the possible interaction between the two spiral galaxies. Another spiral, AM 2356-245 lies 13.8 arcmin away, with a redshift of $z=0.051422 \pm$ 0.000150 (Jones et al., 2009) which shows a one-armed morphology. There are no observable clusters or group within the region at the redshift of ESO 472-G013. 


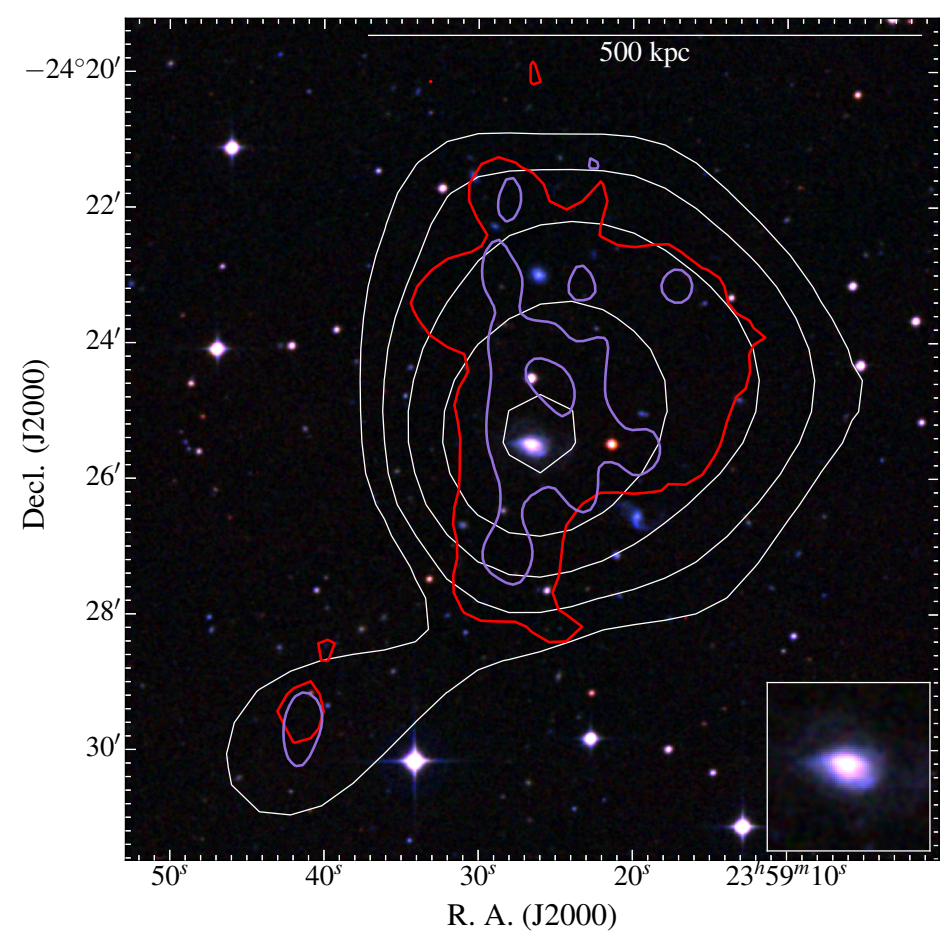

Figure iii.11: RGB image made using the DSS2 IR, red, and blue images with contours overlaid as follows: GLEAM $(200 \mathrm{MHz})$, white, beginning at $20.4 \mathrm{mJy} \mathrm{beam}^{-1}$ and increasing with a factor 2; NVSS, red, a single contour at $1.41 \mathrm{mJy} \mathrm{beam}^{-1}$; TGSS, medium purple, a single contour at $10.5 \mathrm{mJy}_{\text {beam }}{ }^{-1}$. The linear scale is at the redshift of ESO 472-G013. The inset is of ESO 472-G013.

\section{§iii.3.3. Visible and ultraviolet wavelengths}

Fig. iii.11 shows a three-colour DSS2 image with radio contours overlaid. ESO $472-$ G013 is clearly seen at the centre of the image with its companion ESO 471-G012. The spiral nature is not clear from the DSS2 image, instead an irregular morphology is more apparent; deferring classification to the aforementioned publications assuming authors had access to higher fidelity data. If the spiral classification is correct, we see that the lobes of the radio galaxy align approximately perpendicularly to the disk, consistent with previously detected double-lobed spirals. The close companion shows clear barred spiral structure in the DSS2 image, though itself does not feature significant radio emission.

ESO 472-G013 is detected in both the near- and far-ultraviolet (NUV and FUV) bands of the Galaxy Evolution Explorer (GALEX; Martin et al., 2005) All-sky Imaging Survey (AIS). Both ESO 472-G013 and ESO 472-G012 show significant emission in the bands bands, with ESO 472-G012 showing greater intensity in both. We obtain the NUV and FUV magnitudes for ESO 472-G013 from the GALEX AIS catalogue ${ }^{1}: M A G_{\mathrm{NUV}}=19.45 \pm 0.09$ and $M A G_{\mathrm{FUV}}=21.24 \pm 0.26$. We also obtain corresponding flux densities: $S_{\mathrm{NUV}}=60.4 \pm 5.1 \mu \mathrm{Jy}$ and $S_{\mathrm{FUV}}=11.6 \pm 2.8$ $\mathrm{JJy}$.

\footnotetext{
${ }^{1}$ http://galex.stsci.edu/GalexView/
} 


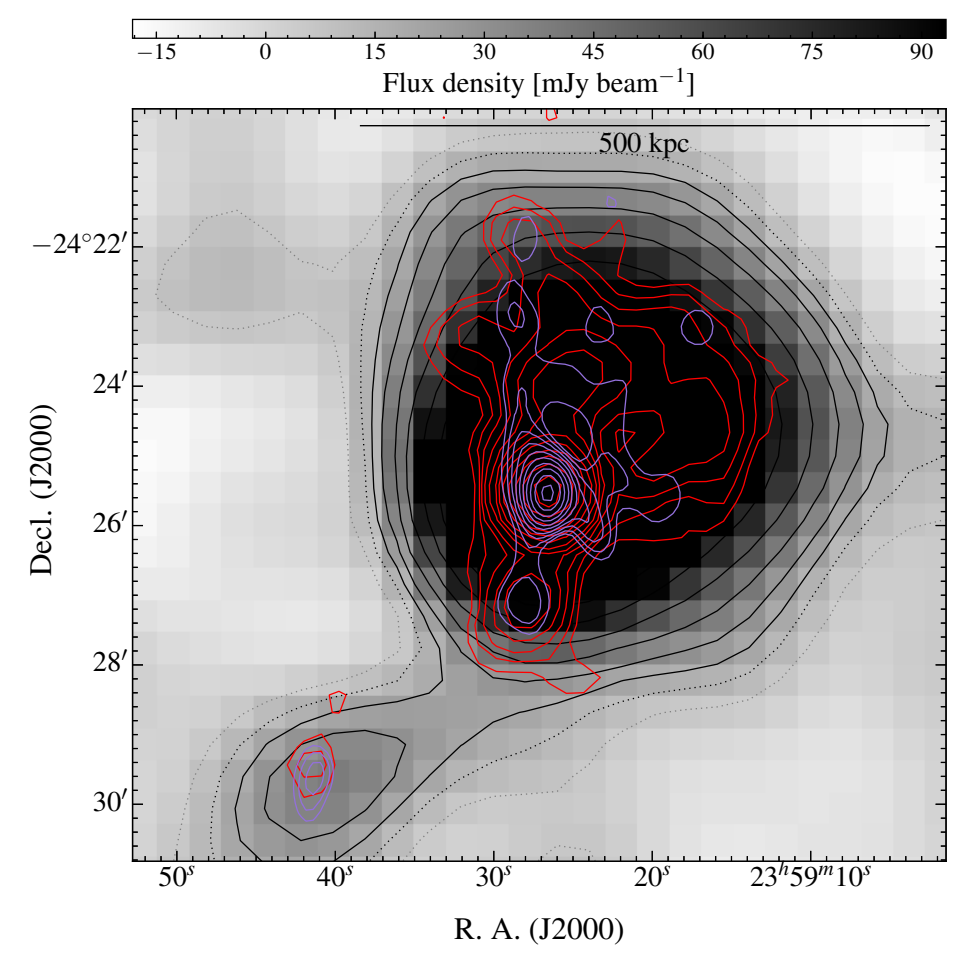

Figure iii.12: GLEAM $200 \mathrm{MHz}$ image of ESO 472-G013 with contours overlaid as follows: GLEAM (200 MHz), black, beginning at $20.4 \mathrm{mJy}^{\text {beam }}{ }^{-1}$; NVSS, red, beginning at $1.41 \mathrm{mJy}^{\text {beam }}{ }^{-1}$; TGSS, medium purple, beginning at $10.5 \mathrm{mJy}^{\text {beam }}{ }^{-1}$. All contours are increasing with a factor of $\sqrt{2}$ and starting at $3 \sigma_{\mathrm{rms}}$. The linear scale is at the redshift of ESO 472-G013.

\section{§iii.3.4. At radio wavelengths}

\section{\$iii.3.4.1. Radio flux density}

The emission was first noticed within the EoR0 field, though we use the GLEAM survey to measure its low-frequency (and resolution) properties. We measure radio flux densities using the custom-built PYTHON code (see Appendix A), which simply sums pixel flux values and corrects for the beam area. An associated error is given as in Eq. ii.1, however for GLEAM at this declination uncertainty in the flux scaling is 8 per cent (Hurley-Walker et al., 2017). Fig. iii.12 shows the GLEAM $200 \mathrm{MHz}$ image with GLEAM, NVSS, and TGSS contours overlaid. Note that despite an expected steeper spectral index for the lobes, the TGSS emission does not trace the entire lobe structure, likely due to lack of sensitivity to flux from extended sources.

We measure the flux density of the combined core and lobe emission at each of the 20 GLEAM narrow bands with the PYTHON code. Prior to this we estimate the noise statistics in each image using BANE. The rms noise of each of the narrow band GLEAM images varies, from 12-65 $\mathrm{mJy}_{\text {beam }}{ }^{-1}$ for the highest- and lowestfrequency bands respectively. In measuring the flux densities, we use a $3 \sigma_{\text {rms }}$ cutoff, where only pixels above this value are included as part of the source. Table iii.5 
Table iii.5: The measured flux densities across the narrow GLEAM bands of the radio emission from ESO 472-G013. The central frequency, $\nu_{c}$, is that given within the FITS header of the GLEAM images, and $\sigma_{\text {rms }}$ for each band is calculated by BANE at the coordinates of ESO 472-G013.

\begin{tabular}{cccc}
\hline \hline Band & $\begin{array}{c}\nu_{\mathrm{c}} \\
(\mathrm{MHz})\end{array}$ & $\begin{array}{c}S_{\nu} \\
(\mathrm{mJy})\end{array}$ & $\begin{array}{c}\sigma_{\text {rms }} \\
\left(\mathrm{mJy} \mathrm{beam}^{-1}\right)\end{array}$ \\
\hline $072-080$ & 76.2 & $1815 \pm 184$ & 65.0 \\
$080-088$ & 87.7 & $1569 \pm 138$ & 30.4 \\
$088-095$ & 83.8 & $1629 \pm 157$ & 49.0 \\
$095-103$ & 91.5 & $1317 \pm 132$ & 45.8 \\
$103-111$ & 99.2 & $1183 \pm 120$ & 40.8 \\
$111-118$ & 106.9 & $1277 \pm 126$ & 38.5 \\
$118-126$ & 118.4 & $1235 \pm 106$ & 16.7 \\
$126-134$ & 114.6 & $1187 \pm 113$ & 31.0 \\
$139-147$ & 122.2 & $1140 \pm 109$ & 29.1 \\
$139-170$ & 129.9 & $961 \pm 93$ & 26.0 \\
$147-154$ & 142.7 & $955 \pm 92$ & 23.9 \\
$154-162$ & 154.2 & $953 \pm 81$ & 11.0 \\
$162-170$ & 150.4 & $858 \pm 82$ & 20.8 \\
$170-177$ & 158.1 & $863 \pm 83$ & 20.2 \\
$177-185$ & 165.8 & $812 \pm 78$ & 18.9 \\
$185-193$ & 173.4 & $808 \pm 77$ & 17.8 \\
$193-200$ & 200.3 & $832 \pm 70$ & 6.8 \\
$200-208$ & 181.1 & $775 \pm 75$ & 18.0 \\
$208-216$ & 188.8 & $734 \pm 70$ & 15.9 \\
$216-223$ & 196.5 & $733 \pm 70$ & 15.1 \\
$223-231$ & 204.2 & $745 \pm 69$ & 14.0 \\
\hline
\end{tabular}


summarises the measured flux densities across the narrow GLEAM bands.

We check the NVSS catalogue (Condon et al., 1998) for flux densities of the emission. The NVSS catalogue breaks the emission into four components: NVSS J235925-242351 and NVSS J235916-242432 for the northern lobe; NVSS J235927242707 for the southern lobe; and NVSS J235926-242530 for the core. The sum of flux densities from these sources is $201.9 \pm 6.9 \mathrm{mJy}$, which we compare to the flux density as measured by the in-house code which gives $194 \pm 3 \mathrm{mJy}$ with a cutoff of $3 \sigma_{\text {rms }}$. The values agree within their uncertainties and we use the lower measured value for the total emission to be consistent with our measured flux densities across the GLEAM bands. We do however use the NVSS catalogue measurement for the core: $87.5 \pm 3.1 \mathrm{mJy}$. We also obtain from the literature the $8.4 \mathrm{GHz}$ flux density of the core from the Combined Radio All-Sky Targeted Eight GHz Survey (CRATES; Healey et al., 2007), and the 147.5 MHz flux density of the core from the TGSS (Intema et al., 2016). Despite the coverage of the VLA Low-frequency Sky Survey (redux, VLSSr; Lane et al., 2014) we do not obtain a flux measurement at 74 $\mathrm{MHz}$ as the flux density scale of the VLSSr below $1 \mathrm{Jy}$ differs sporadically up to approximately 30 per cent.

\section{§iii.3.4.2. Spectral energy distribution from 72-8400 MHz}

We make use of the GLEAM narrow bands and the fine sampling provided over the frequency range $72-231 \mathrm{MHz}$ to estimate the average, integrated spectral index of ESO 472-G013. Due to the low resolution of the GLEAM images in comparison to other surveys such as NVSS and TGSS, we are only able to measure the average spectral index of the entire emission, rather than the core and jets separately. Fig. iii.13 shows the SED between $72-1400 \mathrm{MHz}$, with the $1.4 \mathrm{GHz}$ measurement the sum of the lobes and core components, and the 72-231 MHz GLEAM measurements are of the entire emission. We fit the GLEAM measurements separately as well, which are fit well with a single power law. However, with the inclusion of the $1.4 \mathrm{GHz}$ measurement we find that the simple power law is no longer valid. As the $1.4 \mathrm{GHz}$ measurement is not below the fits of the GLEAM bands, we do not consider that the NVSS imaging has resolved out any of the diffuse emission of the lobes. Instead, we consider that the SED is more complex than a simple power law. The average spectral index is typical of most radio galaxies though the spectral index of the core is lower than average for spirals (e.g. $\langle\alpha\rangle=0.74 \pm 0.12$; Gioia et al., 1982, though this is not for spirals with radio-loud AGN). We find that the core is separated out in the $8.4 \mathrm{GHz}$ or $147.5 \mathrm{MHz}$ measurements of CRATES and the TGSS, as well as the $1.4 \mathrm{GHz}$ measurements of the NVSS. We fit these data to obtain the spectral index of the core of $\alpha_{\text {core }}=-0.555 \pm 0.003$. With this spectral index we calculate the $1.4 \mathrm{GHz}$ power of the core to be $P_{1.4}=(5.21 \pm 0.19) \times 10^{23}$ $\mathrm{W} \mathrm{Hz}{ }^{-1}$.

The top panel of Fig. iii.14 shows the spectral index map of the emission around ESO 472-G013, calculated across the individual narrow GLEAM bands. The bottom panel shows the corresponding spectral index uncertainty map. The spectral 


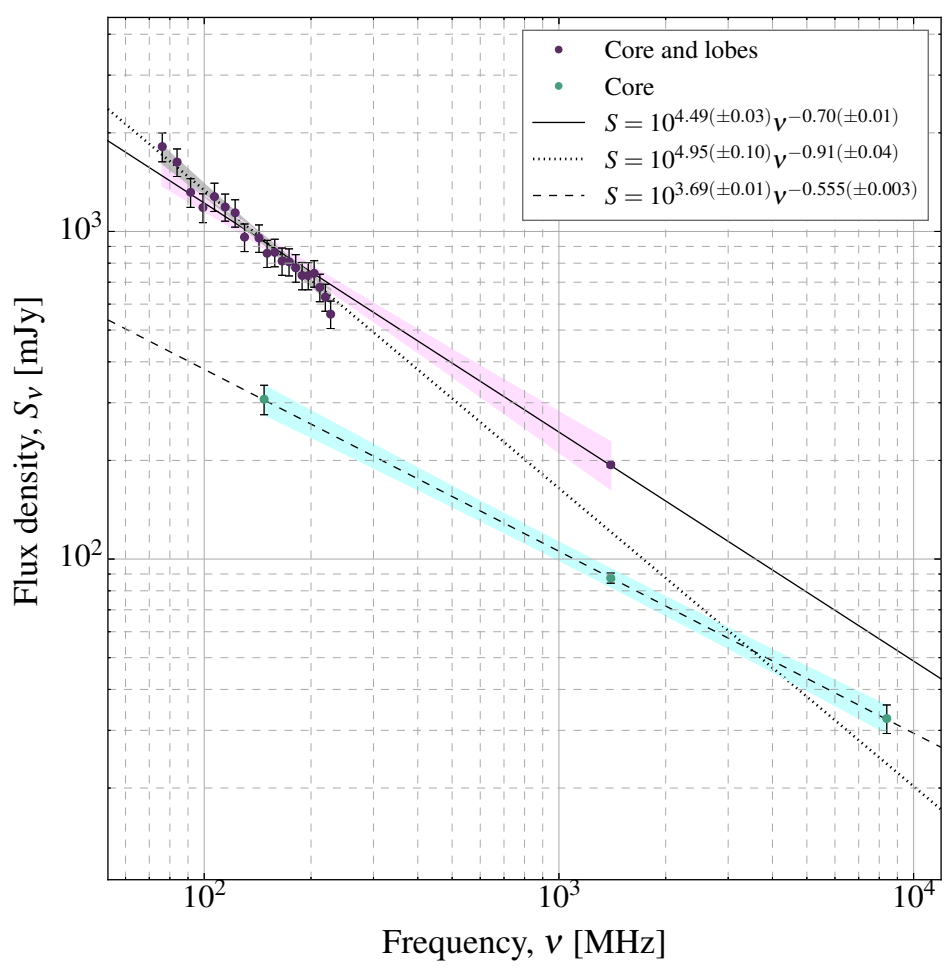

Figure iii.13: Flux density against frequency for the radio emission associated with ESO 472-G013. We show the fit to the GLEAM emission alone (dotted line), as well as the same data with the addition of the $1.4 \mathrm{GHz}$ NVSS point (solid line). This represents the emission from the core and lobes combined. The third fit is to the three measurements of the core only (dashed line). The shaded regions represent 95 per cent confidence intervals for the fits, as in Fig. ii.32. From this we see the GLEAM emission is dominated by the lobes, which have a steeper spectral index than the core. 

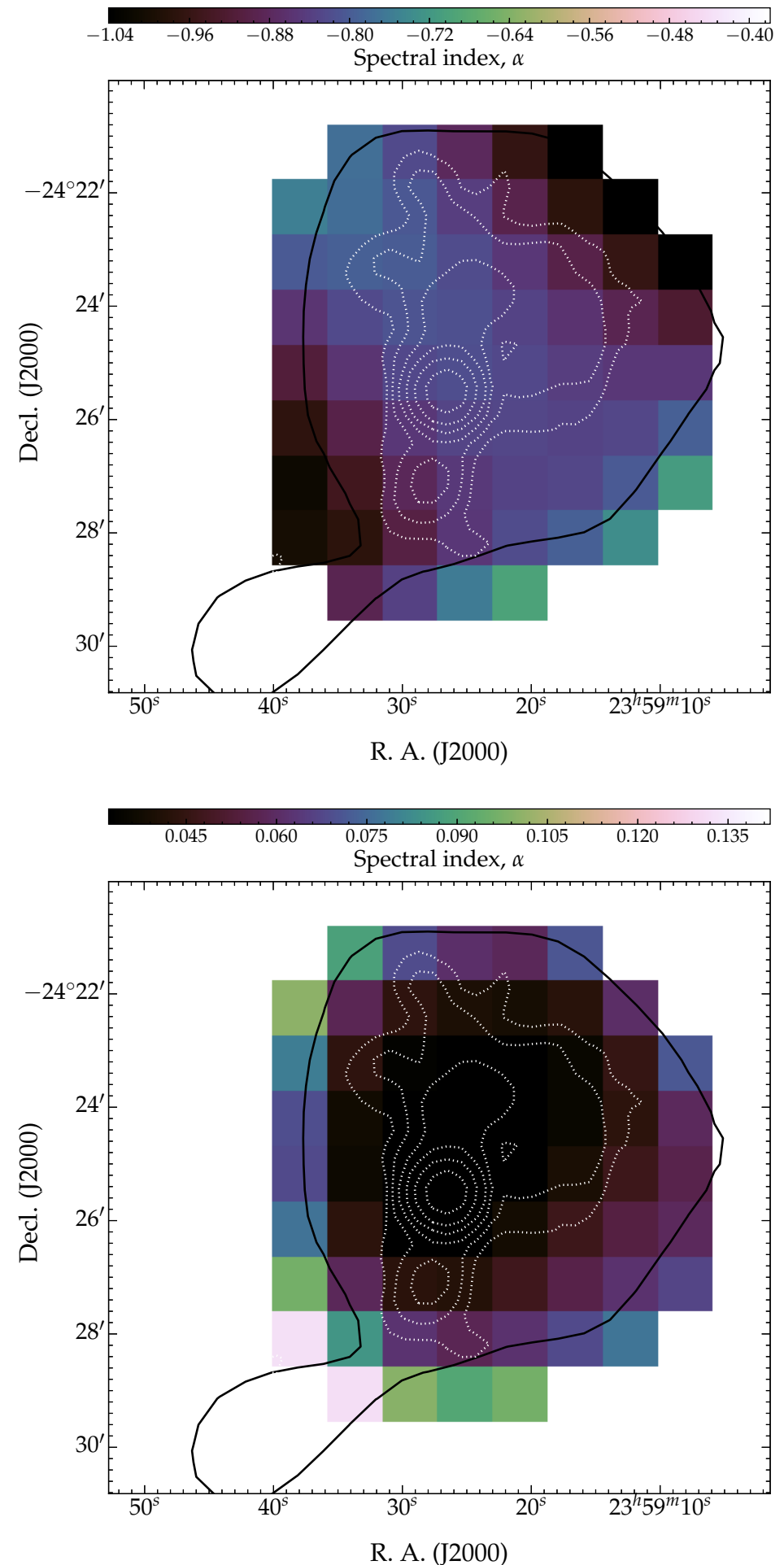

Figure iii.14: Top: The spectral index map of the region around ESO 472-G013 across the 20 narrow GLEAM bands, from 72 to $231 \mathrm{MHz}$. The overlaid white, dotted contours are of the $1.4 \mathrm{GHz}$ NVSS image, starting at $1.5 \mathrm{mJy}^{\mathrm{beam}}{ }^{-1}$ and increasing with a factor of 2 for clarity, and a black, solid contour indicating the $3 \sigma_{\text {rms }}$ level of the $200 \mathrm{MHz}$ GLEAM image. Bottom: As in the top panel except the background is the spectral index uncertainty map. 


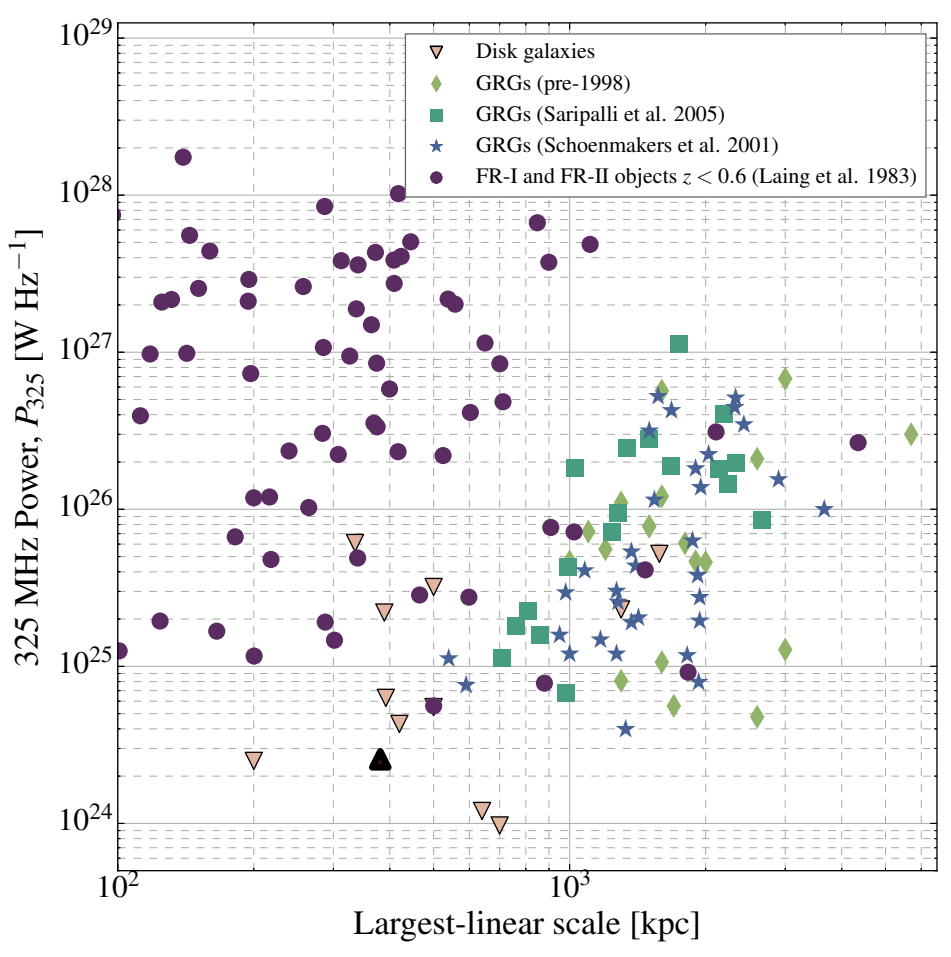

Figure iii.15: $325 \mathrm{MHz}$ rest-frame power against the largest-linear scale for radio galaxies. ESO 472-G013 is represented by an upward-pointing triangle, and other disk galaxies are downward-pointing triangles. The sample of FR-I and FR-II sources shown as circles was compiled by Laing et al. (1983). GRGs compiled by Saripalli et al. (2005) are shown as squares, GRGs reported by Schoenmakers et al. (2001; but see also Schoenmakers et al., 2000), and diamonds are those GRGs compiled in Schoenmakers et al. (2001; see references therein) reported prior to 1998. The cosmology used here is as in Hurley-Walker et al. (2015): a flat $\Lambda$ CDM cosmology with $H_{0}=73 \mathrm{~km} \mathrm{~s}^{-1} \mathrm{Mpc}^{-1}, \Omega_{m}=0.27$, and $\Omega_{\Lambda}=0.73$.

index map is produced by first convolving and regridding GLEAM narrow-band images to the resolution and pixel size of the lowest frequency band $(72-80 \mathrm{MHz})$. This is done using MIRIAD Sault et al. (1995) with tasks CONVOL and REGRID respectively. The FWHM and phase angle for CONVOL are chosen such that the final beam size for the resultant images matched that of the $72-80 \mathrm{MHz}$ band and REGRID used 72-80 MHz band as a template. Due to the low resolution of the 72-80 MHz GLEAM band, the final spectral index map is not able to clearly show the core and lobes as distinct emission, rather blending it all together. Despite this Fig. iii.14 shows steepening towards the edges of the lobes, in the regions farthest from core. This is consistent with typical radio galaxies, where lobes tend towards steeper spectral indices than the cores. The uncertainties in the spectral index calculations increase towards the edges of the emission, and this apparent steepening does not fall outside of these uncertainties. 
Table iii.6: Magnitudes and flux densities as measured by WISE across the four bands, $W 1-4$.

\begin{tabular}{ccccc}
\hline \hline Band & $\begin{array}{c}\lambda \\
(\mu \mathrm{m})\end{array}$ & $\begin{array}{c}M A G \\
(\mathrm{mag})\end{array}$ & $\begin{array}{c}S_{\nu} \\
(\mathrm{mJy})\end{array}$ & $\begin{array}{c}\nu L_{\nu} \\
\left(\times 10^{9} \mathrm{~L}_{\odot}\right)\end{array}$ \\
\hline$W 1$ & 3.4 & $11.407 \pm 0.023$ & $8.31 \pm 0.22$ & - \\
$W 2$ & 4.6 & $11.200 \pm 0.021$ & $5.61 \pm 0.14$ & - \\
$W 3$ & 12 & $8.634 \pm 0.025$ & $9.37 \pm 0.26$ & $3.42 \pm 0.10$ \\
$W 4$ & 22 & $6.876 \pm 0.086$ & $14.6 \pm 1.2$ & $2.90 \pm 0.23$ \\
\hline
\end{tabular}

\section{\$iii.3.4.3. The $\mathrm{P}-\mathrm{D}$ diagram}

We use the spectral index calculated with the GLEAM bands, $\alpha=-0.91 \pm 0.04$, to extrapolate to a $325 \mathrm{MHz}$ rest-frame power of $P_{325}=(2.56 \pm 0.09) \times 10^{24} \mathrm{~W} \mathrm{~Hz}^{-1}$. Fig. iii.15 shows the $325 \mathrm{MHz}$ power against largest-linear size of double-lobed radio sources, adapted from Schoenmakers et al. (2001). We add disk galaxies hosting large-scale radio lobes as in Hurley-Walker et al. (2015), which are shown as down-pointing triangles. We include ESO 472-G013 (up-pointing triangle) and find that the it sits in the low-power, small-size quadrant of the plot. This location is consistent with previously detected disk galaxies hosting large-scale radio lobes which lie between $10^{24}-10^{26} \mathrm{~W} \mathrm{~Hz}^{-1}$ and cover a range of linear sizes and redshifts.

\section{§iii.3.5. At mid-infrared wavelengths}

ESO 472-G013 is detected in the four bands of WISE. The bands $W 1, W 2, W 3$, $W 4$ correspond to $3.4,4.6,12,22 \mu \mathrm{m}$ respectively. We query the AllWISE catalogue (see Cutri et al., 2013) to obtain the $W 1-4$ magnitudes and convert these to flux densities in Jy based on the relation

$$
S_{\nu}=S_{\nu 0} 10^{-0.4 M A G_{\nu}} \quad[\mathrm{Jy}]
$$

where $S_{\nu 0}$ is the frequency-dependent flux density associated with the so-called zero-magnitude, which corresponds to the Vega-calibrated WISE magnitudes, and $M A G$ is the magnitude at a given frequency. A per-band correction is then applied which scales each band based on the in-band spectral index (see Wright et al., 2010; Cutri et al., 2013, though note that notation used here differs). Magnitude measurements are thus converted to flux densities and Table iii.6 summarises the magnitudes, calculated fluxes, and luminosities for selected bands.

\section{§iii.3.6. Star-formation rates}

There exists empirical relationships between infrared, radio, and ultraviolet luminosities of star-forming galaxies and their associated star-formation rates (see e.g. 


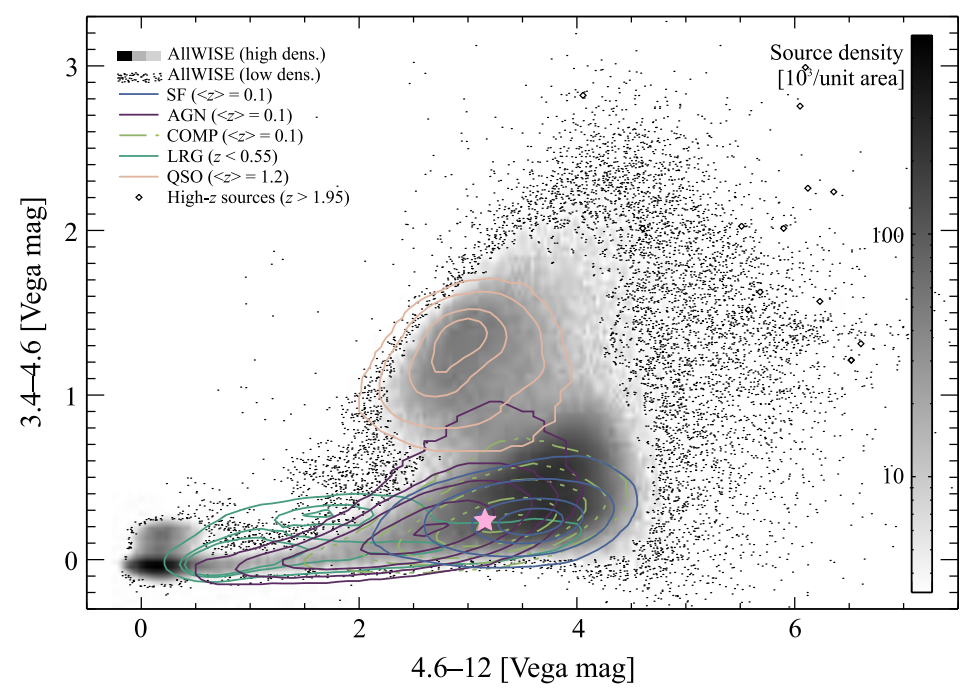

Figure iii.16: The WISE colour-colour plot, taken from Yan et al. (2013) with minor aesthetic editing. We add the location of ESO 472-G013, indicated by a pink star, which finds itself within the intersection of Seyfert AGN and star-forming galaxies (purple and blue contours respectively).

Lequeux 1971, Kennicutt 1983a, Kennicutt 1983b, Condon 1992). In the case of radio, star-formation is related to the radio emission from SNRs and is usually only calculated for stars with masses above $\sim 5 \mathrm{M}_{\odot}$ (see Condon, 1992, for a review). In the far- and mid-infrared, the emission arises due to re-radiation of light absorbed by interstellar dust, likely originating in the optical regime from young stars (Harwit and Pacini, 1975; Johnson et al., 2007), and the ultraviolet traces directly the emission from the same young stars (e.g. Meurer et al., 1995, 1999). SFR measurements between the three methods tend to show deviation, especially when not all radio emission can be attributed to star-formation as in the case of an AGN, where the AGN will cause an overestimate in the radio SFR due to the abundance of synchrotron radiation emitted following the rotation axis of the central black hole. Infrared and ultraviolet may suffer similar overestimates, but these will be much lower than an estimate of the SFR using the $1.4 \mathrm{GHz}$ luminosity for radio-loud AGN.

To determine the SFR of ESO 472-G013, we first look at the infrared WISE images. It is important to note that corrections when converting to flux density from magnitudes are on the order of one part per thousand, and do not substantially affect the estimation of SFR, especially considering the uncertain nature of SFR estimation. Table iii.6 summarises the magnitudes and flux densities of each band. Yan et al. (2013) use colour-colour plots using the WISE bands to explore where different types of galaxies (e.g. star-forming, AGN) lie with respect to each other. Importantly, this gives a qualitative incentive to consider a galaxy as star-forming. We find that ESO 472-G013 has colours $W 1-W 2=0.21 \pm 0.03$ and $W 2-W 3=2.57 \pm 0.03$. Fig. iii.16 shows the location of ESO 472-G013 on the colour-colour diagram of Yan et al. (2013), sitting within the intersection of star-forming and Seyfert galaxies. Thus, we have an incentive to estimate the 
SFR.

To determine the SFR using mid-infrared data, we use the relations presented by Jarrett et al. (2013) (their Equations 1 and 2), which utilise the $W 3$ and $W 4$ bands. These are

$$
\begin{array}{ll}
S F R_{12}=4.91( \pm 0.48) \times 10^{-10} \nu_{12} \frac{L_{12}}{\mathrm{~L}_{\odot}} & {\left[\mathrm{M}_{\odot} \mathrm{yr}^{-1}\right]} \\
S F R_{22}=7.50( \pm 0.08) \times 10^{-10} \nu_{22} \frac{L_{22}}{\mathrm{~L}_{\odot}} & {\left[\mathrm{M}_{\odot} \mathrm{yr}^{-1}\right]}
\end{array}
$$

where the solar luminosity is $L=3.839 \times 10^{33} \mathrm{~W}$. We find that $S F R_{12}=1.68 \pm 0.11$ $\mathrm{M}_{\odot} \mathrm{yr}^{-1}$ and $S F R_{22}=2.17 \pm 0.18 \mathrm{M}_{\odot} \mathrm{yr}^{-1}$. While there is disagreement between these two values, SFR estimations are inherently uncertain, and we consider that this reflects a range of possible values for the true SFR, giving $1.68( \pm 0.11) \leq$ $S F R_{\mathrm{MIR}} \leq 2.17( \pm 0.18) \mathrm{M}_{\odot} \mathrm{yr}^{-1}$ for the mid-infrared SFR. The radio SFR is estimated from the $1.4 \mathrm{GHz}$ flux density of the core only. We use

$$
S F R_{1.4}=\frac{L_{1.4}}{4 \times 10^{21} \mathrm{~W} \mathrm{~Hz}^{-1}} \quad\left[\mathrm{M}_{\odot} \mathrm{yr}^{-1}\right]
$$

from Hopkins (1998) adapted from (Condon, 1992). From the NVSS catalogue, to core is measured to have a flux density of $87.5 \pm 3.1 \mathrm{mJy}$ (Condon et al., 1998) from which we calculate the $1.4 \mathrm{GHz}$ power to be $(5.23 \pm 0.19) \times 10^{23} \mathrm{~W} \mathrm{~Hz}^{-1}$. With this we estimate the radio SFR of $131 \pm 5 \mathrm{M}_{\odot} \mathrm{yr}^{-1}$. This is significantly higher than that calculated with the mid-infrared WISE data though this will be due to the significant radio power contribution of the AGN. Thus $S F R_{1.4}$ is an absolute upper limit to the SFR. Finally, we estimate the SFR from the near-ultraviolet band of the GALEX AIS. This is done through the relation of Schiminovich et al. (2007) adapted from Salim et al. (2007)

$$
S F R_{\mathrm{NUV}}=\frac{L_{\mathrm{NUV}}}{9.26 \times 10^{20} \mathrm{~W} \mathrm{~Hz}^{-1}} \quad\left[\mathrm{M}_{\odot} \mathrm{yr}^{-1}\right]
$$

where NUV is the near-ultraviolet band, 1750-2800 $\AA$. With a NUV luminosity of $(3.51 \pm 0.30) \times 10^{20} \mathrm{~W} \mathrm{~Hz}^{-1}$, this yields $S F R_{\mathrm{NUV}}=0.379 \pm 0.032 \mathrm{M}_{\odot} \mathrm{yr}^{-1}$. This is lower than estimates from the WISE data. However, this is not surprising, as we have not corrected for any attenuation of the NUV band due to dust. This can be corrected for if the attenuation factor is known (or can be calculated, e.g. Johnson et al., 2007). Considering the difference in the NUV and FUV flux densities $(1.79 \pm 0.28)$ we can see from Fig. 13 of Salim et al. (2007) that the attenuation factor is almost certainly $>1$. The attenuation factor cannot be accurately calculated in this case so we consider $S F R_{\mathrm{NUV}}$ to be the absolute lower-limit to the true SFR. Thus, within absolute limits the SFR may lie within $0.379( \pm 0.032) \leq S F R \leq 131( \pm 5) \mathrm{M}_{\odot} \mathrm{yr}^{-1}$. However, due to the power of the radio emission both of the core and the apparent lobes, we can certainly state that the radio power is grossly overestimating $S F R_{1.4}$ due to the presence of a strong AGN. As we cannot determine the AGN contribution, a more reasonable estimate involves only $S F R_{\mathrm{NUV}}$ and $S F R_{\mathrm{MIR}}: 0.379( \pm 0.032) \leq S F R \leq 2.17( \pm 0.18)$ $\mathrm{M}_{\odot} \mathrm{yr}^{-1}$. 


\section{§iii.3.7. Scenarios for the origin of the curious morphology}

Based on the available radio, infrared, and optical data, we consider the following three scenarios for the observed morphology and emission from ESO 472-G013:

- an irregular galaxy with double-lobed structure

- an in-process merger between two galaxies

- a spiral galaxy with a double-lobed structure

In all scenarios, gravitational interaction between ESO 472-G013 and ESO $472-$ G012 may be resulting in the disturbed lobe structure seen, however in the case of an ongoing merger, this may not be necessary. The AGN is likely in its early stages given the spectral index of $\alpha=-0.555 \pm 0.003$ for the core region, and dust is almost certainly present due to the attenuation of the FUV band of GALEX.

In this scenario of an irregular galaxy with double-lobed structure, the irregularity may have been caused by a past merger between two galaxies, at least one of which a spiral. The merger may have triggered a radio-loud AGN, resulting in the radio emission and lobe-structure seen. The irregularity of the galaxy is clear in the DSS2 images, though to confirm this morphology, a higher-resolution follow-up with an optical telescope would be necessary. Irregular galaxies are known to host AGN, usually classed as radio-quiet Seyfert galaxies (Seyfert, 1943) of which spirals are also hosts. This classification is reflected in the WISE colours of Fig. iii.16, which suggest this is a composite Seyfert/star-forming galaxies. However, the radio power of the lobes is sufficient to consider the object as radio-loud (see e.g. Wilson and Colbert, 1995) and given the projected size of $\sim 400 \mathrm{kpc}$ at the redshift of ESO 472-G013 that the classification of Seyfert (or similar radio-quietness) is not correct.

Similar to the previous scenario, we instead consider that the merger is at an earlier stage, with the lobes again being generated by an newly formed AGN, which is consistent with the flat spectral index. In this scenario, the lobes might not be fully-formed, with the potential to increase in size along the lifetime of the AGN perhaps up to the canonical giant radio galaxy (GRG; see e.g. Saripalli et al., 2005) stage with an LLS > $700 \mathrm{kpc}$. This assumes that their projected LLS represents their true LLS. In this scenario is is likely that at least one of the original galaxies was a spiral given the dust present.

A spiral galaxy with double-lobed structure is a possibility, given the literature classifications of ESO 472-G013. In this scenario we are simply seeing a radio-loud spiral galaxy. The galaxy would be similar to those spirals detected by Ledlow et al. (1998); Hota et al. (2011); Bagchi et al. (2014); Mao et al. (2015); Singh et al. (2015), and Mulcahy et al. (2016). Such galaxies are found in underdense environments, e.g. the outskirts of clusters or groups. ESO 472-G013 finds itself with a companion, ESO 472-G012, but also AM 2356-245 approximately 13.8 arcmin away. This environment does not suggest an over-dense region. To explore this scenario, higher-resolution optical imaging will need to be done to see not only the general spiral morphology, but also to determine the orientation of the 
disk. To further complement such an observation, higher-resolution radio imaging, perhaps with the ATCA, might provide a better indication of the orientation of the jets, and the structure of the core.

\section{§iii.3.8. Conclusion}

The galaxy ESO 472-G013 is seen to have a radio-loud AGN at its core, producing large-scale (projected LLS $\sim 400 \mathrm{kpc}$ ) jets which appear to align perpendicularly to the major axis of the galaxy. We show through established WISE colour-colour diagrams that the mid-infrared emission suggests a Seyfert AGN with current star-formation. The infrared data does not suggest the powerful AGN that is observed. We calculate SFR via WISE SFR relationships as well as NUV and 1.4 GHz. The ultraviolet SFR is a lower limit due to dust attenuation, the 1.4 GHz SFR is contaminated by AGN emission, and the MIR SFR is an upper-limit. The somewhat flat spectral index $(\alpha=-0.555 \pm 0.003)$ of the core is suggestive of either new or restarted core activity, which may be implicative of a recent or ongoing merger consistent with an irregular morphology. We suggest that, to confirm the morphology of the host galaxy and the orientation of the jets, follow-up observations would be required. 



\section{§iv. Summary}

During the course of this work, our primary goal was to investigate extended, diffuse emission associated with galaxy clusters within a 45 degree by 45 degree radio image centred on $\mathrm{R}$. A. $=0^{\circ}$, decl. $=-27^{\circ}$. The image has a central frequency of $168 \mathrm{MHz}$ and a resolution of $\sim 2.3$ arcmin with a sensitivity of up to $\sim 2.3 \mathrm{mJy}_{\text {beam }}{ }^{-1}$. The types of emission most interesting to us were radio haloes, relics, and phoenices; emission from relativistic electrons in cluster magnetic fields, associated with the ICM rather than a particular galaxy within a cluster.

In doing so, we have identified, classified and measured $168 \mathrm{MHz}$ radio properties, where possible, of a number of diffuse radio sources in the field including 11 haloes, 9 relics, and 3 phoenices. A subset of these are new discoveries and add to the slowly growing sample of such emission. We update the known $P_{1.4}-L_{\mathrm{X}}$ and $P_{1.4}-M$ relations between radio halo power and cluster X-ray luminosity and mass to find that an increase in sample size greater than a factor of two has yielded consistent fitting parameters to the relations. We see that the detection limit, derived from the $P_{1.4}-M$ relation, of radio haloes with the EoR0 field is upheld, and we find that the MWA is able to push into higher-redshift, lower-mass clusters. However we note that the MWA is still limited by its resolution, which is a function of its current longest baseline of $\sim 3 \mathrm{~km}$, and we still require high-resolution follow-up on a number of ambiguous and otherwise hard to classify steep-spectrum emission that has been detected as part of this work.

As part of follow-up work, we made use of DDT to image the cluster Abell S1136 with the ATCA at $2.1 \mathrm{GHz}$ in the hopes of detecting core emission from a long-dead radio galaxy associated with ESO 470-G020 - the BCG of the cluster. We detected the steep-spectrum, remnant lobes at $168 \mathrm{MHz}$ and within the TGSS images at $147.5 \mathrm{MHz}$, and provided follow-up spectral analysis with GLEAM, providing a spectral index of $\alpha_{118}^{231}=-1.81 \pm 0.12$ averaged over the entire emission. In the ATCA image we detect a faint core and measure a spectral index of $\alpha_{1413}^{2274}=$ $-1.39 \pm 0.55$ which has a large uncertainty due to the low-brightness of the emission. We also find a number of faint point sources embedded as well, though do not provide spectral indices due to their low signal-to-noise ratios.

The cluster Abell S1063 was analysed with archival ATCA data, within which we hoped to find a massive diffuse halo but only radio galaxies were clearly detected in the ATCA images, which had sensitivity to structures up to 21 arcmin. The rms noise in the stacked ATCA image at $2.1 \mathrm{GHz}$ was $18 \mu \mathrm{Jy}$. We had expected to see a halo in this cluster due to the morphology of the blended sources at 168 $\mathrm{MHz}$ but also due to the cluster's massive and X-ray luminous nature.

Finally, ESO 472-G013, a disk galaxy hosting large-scale radio lobes, is investigated after being detected within the EoR0 field. We measure a spectral index across the narrow GLEAM bands of $\alpha_{72}^{231}=-0.91 \pm 0.04$ for the lobes and core 
combined. We note that the core alone has a spectral index, from higher-frequency, higher-resolution imaging, of $\alpha_{\text {core }}=-0.555 \pm 0.003$. In comparison with WISE colour-colour plots we consider that ESO 472-G013 is likely star-forming, and estimate its star-formation rate via its WISE MIR, GALEX NUV, and $1.4 \mathrm{GHz}$ properties. We see that the $1.4 \mathrm{GHz} \mathrm{SFR}$ is overestimated due to the presence of a radio-loud AGN. We compare ESO 472-G013 to other disk galaxies hosting large-scale radio lobes and other radio galaxies within the the context of their 325 $\mathrm{MHz}$ power and LLS measurements, finding that ESO 472-G013 lies within the space occupied by similar disk galaxies. Lastly, we briefly consider the origins of the morphology and AGN activity of ESO 472-G013, considering a number of scenarios that might have led to its current state. 


\section{Bibliography}

Abdo, A. A., Ackermann, M., Ajello, M., et al. (2009). Radio-Loud NarrowLine Seyfert 1 as a New Class of Gamma-Ray Active Galactic Nuclei. The Astrophysical Journal Letters, 707:L142-L147.

Abell, G. O., Corwin, Jr., H. G., and Olowin, R. P. (1989). A catalog of rich clusters of galaxies. The Astrophysical Journal Supplementary Series, 70:1138.

Akritas, M. G. and Bershady, M. A. (1996). Linear Regression for Astronomical Data with Measurement Errors and Intrinsic Scatter. The Astrophysical Journal, 470:706.

Ali, Z. S., Parsons, A. R., Zheng, H., et al. (2015). PAPER-64 Constraints on Reionization: The $21 \mathrm{~cm}$ Power Spectrum at $\mathrm{z}=8.4$. The Astrophysical Journal, 809:61.

Alonso, M. V., Valotto, C., Lambas, D. G., et al. (1999). Velocity dispersion estimates of APM galaxy clusters. Mon. Not. R. Astron. Soc., 308:618-622.

Alvarez, H., Aparici, J., May, J., et al. (2000). The radio continuum spectrum of Centaurus A's large-scale components. Astronomy \&5 Astrophysics, 355:863872.

Baade, W. and Minkowski, R. (1954). On the Identification of Radio Sources. The Astrophysical Journal, 119:215.

Bagchi, J., Durret, F., Neto, G. B. L., et al. (2006). Giant Ringlike Radio Structures Around Galaxy Cluster Abell 3376. Science, 314:791-794.

Bagchi, J., Vivek, M., Vikram, V., et al. (2014). Megaparsec Relativistic Jets Launched from an Accreting Supermassive Black Hole in an Extreme Spiral Galaxy. The Astrophysical Journal, 788:174.

Balick, B. and Brown, R. L. (1974). Intense sub-arcsecond structure in the galactic center. The Astrophysical Journal, 194:265-270.

Batuski, D. J., Miller, C. J., Slinglend, K. A., et al. (1999). Discovery of Extreme Examples of Superclustering in Aquarius. The Astrophysical Journal, 520:491-506.

Bernardi, G., Venturi, T., Cassano, R., et al. (2016). KAT-7 observations of an unbiased sample of mass-selected galaxy clusters. Mon. Not. R. Astron. Soc., 456:1259-1268.

Blandford, R. D. and Rees, M. J. (1974). A 'twin-exhaust' model for double radio sources. Mon. Not. R. Astron. Soc., 169:395-415.

Blasi, P. and Colafrancesco, S. (1999). Cosmic rays, radio halos and nonthermal X-ray emission in clusters of galaxies. Astroparticle Physics, 12:169-183. 
Bleem, L. E., Stalder, B., de Haan, T., et al. (2015). Galaxy Clusters Discovered via the Sunyaev-Zel'dovich Effect in the 2500-Square-Degree SPT-SZ Survey. The Astrophysical Journal Supplementary Series, 216:27.

Bock, D. C.-J., Large, M. I., and Sadler, E. M. (1999). SUMSS: A Wide-Field Radio Imaging Survey of the Southern Sky. I. Science Goals, Survey Design, and Instrumentation. The Astronomical Journal, 117:1578-1593.

Böhringer, H., Schuecker, P., Guzzo, L., et al. (2004). The ROSAT-ESO Flux Limited X-ray (REFLEX) Galaxy cluster survey. V. The cluster catalogue. Astronomy \& Astrophysics, 425:367-383.

Böhringer, H., Schuecker, P., Pratt, G. W., et al. (2007). The representative XMMNewton cluster structure survey (REXCESS) of an X-ray luminosity selected galaxy cluster sample. Astronomy \& Astrophysics, 469:363-377.

Bolton, J. G., Stanley, G. J., and Slee, O. B. (1949). Positions of Three Discrete Sources of Galactic Radio-Frequency Radiation. Nature, 164:101-102.

Bonafede, A., Feretti, L., Murgia, M., et al. (2010). The Coma cluster magnetic field from Faraday rotation measures. Astronomy \& Astrophysics, 513:A30.

Bowman, J. D., Cairns, I., Kaplan, D. L., et al. (2013). Science with the Murchison Widefield Array. Publications of the Astron. Soc. of Australia, 30:e031.

Bravi, L., Gitti, M., and Brunetti, G. (2016). Do radio mini-halos and gas heating in cool-core clusters have a common origin? Mon. Not. R. Astron. Soc., 455:L41-L45.

Briggs, D. S. (1995). High Fidelity Interferometric Imaging: Robust Weighting and NNLS Deconvolution. In American Astronomical Society Meeting Abstracts, volume 27 of Bulletin of the American Astronomical Society, p. 1444.

Brown, R. L. (1982). Precessing jets in Sagittarius A - Gas dynamics in the central parsec of the galaxy. The Astrophysical Journal, 262:110-119.

Brown, S., Duesterhoeft, J., and Rudnick, L. (2011). Multiple Shock Structures in a Radio-selected Cluster of Galaxies. The Astrophysical Journal Letters, 727:L25.

Brunetti, G. and Blasi, P. (2005). Alfvénic reacceleration of relativistic particles in galaxy clusters in the presence of secondary electrons and positrons. Mon. Not. R. Astron. Soc., 363:1173-1187.

Brunetti, G. and Lazarian, A. (2011). Particle reacceleration by compressible turbulence in galaxy clusters: effects of a reduced mean free path. Mon. Not. R. Astron. Soc., 412:817-824.

Brunetti, G. and Lazarian, A. (2016). Stochastic reacceleration of relativistic electrons by turbulent reconnection: a mechanism for cluster-scale radio emission? Mon. Not. R. Astron. Soc., 458:2584-2595.

Brunetti, G., Setti, G., Feretti, L., et al. (2001). Particle reacceleration in the Coma cluster: radio properties and hard X-ray emission. Mon. Not. R. Astron. Soc., 320:365-378. 
Buote, D. A. (2001). On the Origin of Radio Halos in Galaxy Clusters. The Astrophysical Journal Letters, 553:L15-L18.

Burbidge, G. R. and Burbidge, E. M. (1957). The Sources of Radio Emission in NGC 5128 and NGC 1316. The Astrophysical Journal, 125:1.

Callingham, J. R., Ekers, R. D., Gaensler, B. M., et al. (2017). Extragalactic Peaked-Spectrum Radio Sources at Low Frequencies. ArXiv e-prints.

Cantwell, T. M., Scaife, A. M. M., Oozeer, N., et al. (2016). A newly discovered radio halo in merging cluster MACS J2243.3-0935. Mon. Not. R. Astron. Soc., 458:1803-1814.

Caretta, C. A., Maia, M. A. G., Kawasaki, W., et al. (2002). The Aquarius Superclusters. I. Identification of Clusters and Superclusters. The Astronomical Journal, 123:1200-1215.

Caretta, C. A., Maia, M. A. G., and Willmer, C. N. A. (2004). The Aquarius Superclusters. II. Spectroscopic and Photometric Data. The Astronomical Journal, 128:2642-2651.

Cassano, R., Brunetti, G., Norris, R. P., et al. (2012). Radio halos in future surveys in the radio continuum. Astronomy \& Astrophysics, 548:A100.

Cassano, R., Brunetti, G., and Setti, G. (2006). Statistics of giant radio haloes from electron reacceleration models. Mon. Not. R. Astron. Soc., 369:15771595 .

Cassano, R., Ettori, S., Brunetti, G., et al. (2013). Revisiting Scaling Relations for Giant Radio Halos in Galaxy Clusters. The Astrophysical Journal, 777:141.

Castellano, M., Amorín, R., Merlin, E., et al. (2016). The ASTRODEEP Frontier Fields catalogues. II. Photometric redshifts and rest frame properties in Abell2744 and MACS-J0416. Astronomy \& Astrophysics, 590:A31.

Chiaberge, M. and Marconi, A. (2011). On the origin of radio loudness in active galactic nuclei and its relationship with the properties of the central supermassive black hole. Mon. Not. R. Astron. Soc., 416:917-926.

Chon, G. and Böhringer, H. (2012). The ROSAT-ESO flux limited X-ray galaxy cluster survey (REFLEX II). I. Newly identified X-ray luminous clusters at $z \geq 0.2$. Astronomy \& Astrophysics, 538:A35.

Clark, B. G. (1980). An efficient implementation of the algorithm 'CLEAN'. Astronomy $\&$ Astrophysics, 89:377.

Clarke, T. E., Kronberg, P. P., and Böhringer, H. (2001). A New Radio-X-Ray Probe of Galaxy Cluster Magnetic Fields. The Astrophysical Journal Letters, 547:L111-L114.

Cohen, A. S. and Clarke, T. E. (2011). An Ultra-steep-spectrum Radio Relic in the Galaxy Cluster A2443. The Astronomical Journal, 141:149.

Condon, J. J. (1992). Radio emission from normal galaxies. Annual Review of Astronomy \& Astrophysics, 30:575-611. 
Condon, J. J., Cotton, W. D., Greisen, E. W., et al. (1998). The NRAO VLA Sky Survey. The Astronomical Journal, 115:1693-1716.

Cordey, R. A. (1986). Radio sources in giant E and S0 galaxies. Mon. Not. R. Astron. Soc., 219:575-588.

Cordey, R. A. (1987). IC 2476 - A possible relic radio galaxy. Mon. Not. R. Astron. Soc., 227:695-700.

Coziol, R., Andernach, H., Caretta, C. A., et al. (2009). The Dynamical State of Brightest Cluster Galaxies and The Formation of Clusters. The Astronomical Journal, 137:4795-4809.

Cutri, R. M., Wright, E. L., Conrow, T., et al. (2013). Explanatory Supplement to the AllWISE Data Release Products. Technical report, University of California, Los Angeles, and the Jet Propulsion Laboratory/California Institute of Technology.

Dahle, H., Kaiser, N., Irgens, R. J., et al. (2002). Weak Gravitational Lensing by a Sample of X-Ray Luminous Clusters of Galaxies. I. The Data Set. The Astrophysical Journal Supplementary Series, 139:313-368.

Dalton, G. B., Efstathiou, G., Maddox, S. J., et al. (1994). The APM Galaxy Survey - Part Four - Redshifts of Rich Clusters of Galaxies. Mon. Not. R. Astron. Soc., 269:151.

Dalton, G. B., Maddox, S. J., Sutherland, W. J., et al. (1997). The APM Galaxy Survey - V. Catalogues of galaxy clusters. Mon. Not. R. Astron. Soc., 289:263-284.

de Gasperin, F., Intema, H. T., van Weeren, R. J., et al. (2015). A powerful double radio relic system discovered in PSZ1 G108.18-11.53: evidence for a shock with non-uniform Mach number? Mon. Not. R. Astron. Soc., 453:3483-3498.

de Gasperin, F., van Weeren, R. J., Brüggen, M., et al. (2014). A new double radio relic in PSZ1 G096.89+24.17 and a radio relic mass-luminosity relation. Mon. Not. R. Astron. Soc., 444:3130-3138.

de Propris, R., Stanford, S. A., Eisenhardt, P. R., et al. (1999). The K-Band Luminosity Function in Galaxy Clusters to $z \sim 1$. The Astronomical Journal, 118:719-729.

Dehghan, S., Johnston-Hollitt, M., Franzen, T. M. O., et al. (2014). Bent-tailed Radio Sources in the Australia Telescope Large Area Survey of the Chandra Deep Field South. The Astronomical Journal, 148:75.

Dennison, B. (1980). Formation of radio halos in clusters of galaxies from cosmicray protons. The Astrophysical Journal Letters, 239:L93-L96.

di Nella, H., Couch, W. J., Paturel, G., et al. (1996). Are the Perseus-Pisces chain and the Pavo-Indus wall connected? Mon. Not. R. Astron. Soc., 283:367-380.

Diego, J. M., Broadhurst, T., Wong, J., et al. (2016). A free-form mass model of the Hubble Frontier Fields cluster AS1063 (RXC J2248.7-4431) with over one hundred constraints. Mon. Not. R. Astron. Soc., 459:3447-3459. 
Doeleman, S. S., Weintroub, J., Rogers, A. E. E., et al. (2008). Event-horizon-scale structure in the supermassive black hole candidate at the Galactic Centre. Nature, 455:78-80.

Dolag, K. and Enßlin, T. A. (2000). Radio halos of galaxy clusters from hadronic secondary electron injection in realistic magnetic field configurations. Astronomy \& Astrophysics, 362:151-157.

Dreyer, J. L. E. (1888). A New General Catalogue of Nebulæ and Clusters of Stars, being the Catalogue of the late Sir John F. W. Herschel, Bart, revised, corrected, and enlarged. Memoires of the Royal Astronomical Society, 49:1.

Ebeling, H., Edge, A. C., Bohringer, H., et al. (1998). The ROSAT Brightest Cluster Sample - I. The compilation of the sample and the cluster log N-log S distribution. Mon. Not. R. Astron. Soc., 301:881-914.

Ebeling, H., Edge, A. C., Mantz, A., et al. (2010). The X-ray brightest clusters of galaxies from the Massive Cluster Survey. Mon. Not. R. Astron. Soc., 407:83-93.

Enßlin, T. A. and Brüggen, M. (2002). On the formation of cluster radio relics. Mon. Not. R. Astron. Soc., 331:1011-1019.

Enßlin, T. A. and Gopal-Krishna (2001). Reviving fossil radio plasma in clusters of galaxies by adiabatic compression in environmental shock waves. Astronomy E Astrophysics, 366:26-34.

Fanti, C., Fanti, R., Gioia, I. M., et al. (1977). Observations of 40 low luminosity radio galaxies with the Westerbork Synthesis Radio Telescope. Astronomy 86 Astrophysics Suppl. Series, 29:279-292.

Feretti, L., Giovannini, G., Govoni, F., et al. (2012). Clusters of galaxies: observational properties of the diffuse radio emission. Astronomy $\& 5$ Astrophysics Reviews, 20:54.

Finlay, E. A. and Jones, B. B. (1973). Measurements of source strengths at 29.9 MHz. Australian Journal of Physics, 26:389.

Franzen, T. M. O., Jackson, C. A., Offringa, A. R., et al. (2016). The $154 \mathrm{MHz}$ radio sky observed by the Murchison Widefield Array: noise, confusion, and first source count analyses. Mon. Not. R. Astron. Soc., 459:3314-3325.

Frater, R. H., Brooks, J. W., and Whiteoak, J. B. (1992). The Australia Telescope - Overview. Journal of Electrical and Electronics Engineering Australia, 12:103-112.

Fujita, Y., Sarazin, C. L., Kempner, J. C., et al. (2002). Chandra Observations of the Disruption of the Cool Core in A133. The Astrophysical Journal, 575:764778 .

Fujita, Y., Sarazin, C. L., Reiprich, T. H., et al. (2004). XMM-Newton Observations of A133: A Weak Shock Passing through the Cool Core. The Astrophysical Journal, 616:157-168. 
Gallimore, J. F., Axon, D. J., O’Dea, C. P., et al. (2006). A Survey of KiloparsecScale Radio Outflows in Radio-Quiet Active Galactic Nuclei. The Astronomical Journal, 132:546-569.

Gioia, I. M., Gregorini, L., and Klein, U. (1982). High frequency radio continuum observations of bright spiral galaxies. Astronomy \&6 Astrophysics, 116:164174.

Giovannini, G. and Feretti, L. (2000). Halo and relic sources in clusters of galaxies. New Astronomy, 5:335-347.

Giovannini, G., Feretti, L., Gregorini, L., et al. (1988). Radio nuclei in elliptical galaxies. Astronomy \& Astrophysics, 199:73-84.

Giovannini, G., Tordi, M., and Feretti, L. (1999). Radio halo and relic candidates from the NRAO VLA Sky Survey. New Astronomy, 4:141-155.

Girardi, M., Boschin, W., Gastaldello, F., et al. (2016). A multiwavelength view of the galaxy cluster Abell 523 and its peculiar diffuse radio source. Mon. Not. R. Astron. Soc., 456:2829-2847.

Gómez, P. L., Valkonen, L. E., Romer, A. K., et al. (2012). Optical and X-Ray Observations of the Merging Cluster AS1063. The Astronomical Journal, 144:79.

Govoni, F., Feretti, L., Giovannini, G., et al. (2001). Radio and X-ray diffuse emission in six clusters of galaxies. Astronomy \& Astrophysics, 376:803-819.

Govoni, F., Murgia, M., Xu, H., et al. (2013). Polarization of cluster radio halos with upcoming radio interferometers. Astronomy \& Astrophysics, 554:A102.

Green, D. A. (2011). A colour scheme for the display of astronomical intensity images. Bulletin of the Astronomical Society of India, 39:289-295.

Gunn, J. E. and Gott, III, J. R. (1972). On the Infall of Matter Into Clusters of Galaxies and Some Effects on Their Evolution. The Astrophysical Journal, 176:1.

Guzzo, L., Schuecker, P., Böhringer, H., et al. (2009). The REFLEX galaxy cluster survey. VIII. Spectroscopic observations and optical atlas,. Astronomy 86 Astrophysics, 499:357-369.

Hales, S. E. G., Mayer, C. J., Warner, P. J., et al. (1991). The 6C survey of radio sources. IV - The zone delta from 67 to $82 \mathrm{deg}$, alpha from 0 to $24 \mathrm{H}$. Mon. Not. R. Astron. Soc., 251:46-53.

Hambly, N. C., Davenhall, A. C., Irwin, M. J., et al. (2001a). The SuperCOSMOS Sky Survey - III. Astrometry. Mon. Not. R. Astron. Soc., 326:1315-1327.

Hambly, N. C., Irwin, M. J., and MacGillivray, H. T. (2001b). The SuperCOSMOS Sky Survey - II. Image detection, parametrization, classification and photometry. Mon. Not. R. Astron. Soc., 326:1295-1314.

Hambly, N. C., MacGillivray, H. T., Read, M. A., et al. (2001c). The SuperCOSMOS Sky Survey - I. Introduction and description. Mon. Not. R. Astron. Soc., 326:1279-1294. 
Hancock, P. J., Murphy, T., Gaensler, B. M., et al. (2012). Compact continuum source finding for next generation radio surveys. Mon. Not. R. Astron. Soc., 422:1812-1824.

Hao, J., McKay, T. A., Koester, B. P., et al. (2010). A GMBCG Galaxy Cluster Catalog of 55,424 Rich Clusters from SDSS DR7. The Astrophysical Journal Supplementary Series, 191:254-274.

Harwit, M. and Pacini, F. (1975). Infrared galaxies - Evolutionary stages of massive star formation. The Astrophysical Journal Letters, 200:L127-L129.

Healey, S. E., Romani, R. W., Taylor, G. B., et al. (2007). CRATES: An All-Sky Survey of Flat-Spectrum Radio Sources. The Astrophysical Journal Supplementary Series, 171:61-71.

Henley, D. B. and Shelton, R. L. (2013). An XMM-Newton Survey of the Soft XRay Background. III. The Galactic Halo X-Ray Emission. The Astrophysical Journal, 773:92.

Herbig, T. and Birkinshaw, M. (1994). Discovery of a Radio Halo Source in Abell 2163. In American Astronomical Society Meeting Abstracts, volume 26 of Bulletin of the American Astronomical Society, p. 1403.

Hewish, A., Bell, S. J., Pilkington, J. D. H., et al. (1968). Observation of a Rapidly Pulsating Radio Source. Nature, 217:709-713.

Hicks, A. K., Pratt, G. W., Donahue, M., et al. (2013). The X-ray properties of optically selected clusters of galaxies. Mon. Not. R. Astron. Soc., 431:25422553.

Hoekstra, H., Herbonnet, R., Muzzin, A., et al. (2015). The Canadian Cluster Comparison Project: detailed study of systematics and updated weak lensing masses. Mon. Not. R. Astron. Soc., 449:685-714.

Högbom, J. A. (1974). Aperture Synthesis with a Non-Regular Distribution of Interferometer Baselines. Astronomy \& Astrophysics Suppl. Series, 15:417.

Hogg, D. W. (1999). Distance measures in cosmology. ArXiv Astrophysics e-prints.

Hogg, D. W., Baldry, I. K., Blanton, M. R., et al. (2002). The K correction. ArXiv Astrophysics e-prints.

Hogg, D. W., Bovy, J., and Lang, D. (2010). Data analysis recipes: Fitting a model to data. ArXiv e-prints.

Hopkins, A. M. (1998). The Phoenix Multiwavelength Deep Survey. Ph.D. thesis, School of Physics, University of Sydney, NSW, 2006, Australia.

Hopkins, P. F., Hernquist, L., Cox, T. J., et al. (2006). A Unified, Merger-driven Model of the Origin of Starbursts, Quasars, the Cosmic X-Ray Background, Supermassive Black Holes, and Galaxy Spheroids. The Astrophysical Journal Supplementary Series, 163:1-49.

Hota, A., Sirothia, S. K., Ohyama, Y., et al. (2011). Discovery of a spiral-host episodic radio galaxy. Mon. Not. R. Astron. Soc., 417:L36-L40. 
Hurley-Walker, N., Callingham, J. R., Hancock, P. J., et al. (2017). GaLactic and Extragalactic All-sky Murchison Widefield Array (GLEAM) survey - I. A low-frequency extragalactic catalogue. Mon. Not. R. Astron. Soc., 464:11461167.

Hurley-Walker, N., Johnston-Hollitt, M., Ekers, R., et al. (2015). Serendipitous discovery of a dying Giant Radio Galaxy associated with NGC 1534, using the Murchison Widefield Array. Mon. Not. R. Astron. Soc., 447:2468-2478.

Intema, H. T., Jagannathan, P., Mooley, K. P., et al. (2016). The GMRT 150 MHz All-sky Radio Survey: First Alternative Data Release TGSS ADR1. ArXiv e-prints.

Jackson, J. D. (1975). Classical electrodynamics. New York: Wiley, 1975, 2nd ed.

Jaffe, W. J. (1977). Origin and transport of electrons in the halo radio source in the Coma cluster. The Astrophysical Journal, 212:1-7.

Jaffe, W. J. and Perola, G. C. (1973). Dynamical Models of Tailed Radio Sources in Clusters of Galaxies. Astronomy \& Astrophysics, 26:423.

Jarrett, T. H., Masci, F., Tsai, C. W., et al. (2013). Extending the Nearby Galaxy Heritage with WISE: First Results from the WISE Enhanced Resolution Galaxy Atlas. The Astronomical Journal, 145:6.

Johnson, B. D., Schiminovich, D., Seibert, M., et al. (2007). Ultraviolet through Infrared Spectral Energy Distributions from 1000 SDSS Galaxies: Dust Attenuation. The Astrophysical Journal Supplementary Series, 173:392-403.

Johnston-Hollitt, M. (2003). Detection of magnetic fields and diffuse radio emission in Abell 3667 and other rich southern clusters of galaxies. Ph.D. thesis, University of Adelaide.

Jones, C. and Forman, W. (1999). Einstein Observatory Images of Clusters of Galaxies. The Astrophysical Journal, 511:65-83.

Jones, D. H., Read, M. A., Saunders, W., et al. (2009). The 6dF Galaxy Survey: final redshift release (DR3) and southern large-scale structures. Mon. Not. R. Astron. Soc., 399:683-698.

Jones, D. H., Saunders, W., Colless, M., et al. (2004). The 6dF Galaxy Survey: samples, observational techniques and the first data release. Mon. Not. R. Astron. Soc., 355:747-763.

Kale, R. and Dwarakanath, K. S. (2012). Multi-frequency Studies of Radio Relics in the Galaxy Clusters A4038, A1664, and A786. The Astrophysical Journal, 744:46.

Kale, R., Venturi, T., Giacintucci, S., et al. (2013). The Extended GMRT Radio Halo Survey. I. New upper limits on radio halos and mini-halos. Astronomy \& Astrophysics, 557:A99.

Kale, R., Venturi, T., Giacintucci, S., et al. (2015). The Extended GMRT Radio Halo Survey. II. Further results and analysis of the full sample. Astronomy $\&$ Astrophysics, 579:A92. 
Kempner, J. C., Blanton, E. L., Clarke, T. E., et al. (2004). Conference Note: A Taxonomy of Extended Radio Sources in Clusters of Galaxies. In Reiprich, T., Kempner, J., and Soker, N., eds., The Riddle of Cooling Flows in Galaxies and Clusters of galaxies.

Kempner, J. C., Sarazin, C. L., and Ricker, P. M. (2002). Chandra Observations of A85: Merger of the South Subcluster. The Astrophysical Journal, 579:236246.

Kennicutt, R. (1983a). The origin of the nonthermal radio emission in normal disk galaxies. Astronomy \& Astrophysics, 120:219-222.

Kennicutt, Jr., R. C. (1983b). The rate of star formation in normal disk galaxies. The Astrophysical Journal, 272:54-67.

Knowles, K., Intema, H. T., Baker, A. J., et al. (2016). A giant radio halo in a low-mass SZ-selected galaxy cluster: ACT-CL J0256.5+0006. Mon. Not. R. Astron. Soc., 459:4240-4258.

Komissarov, S. S. and Gubanov, A. G. (1994). Relic radio galaxies: evolution of synchrotron spectrum. Astronomy $\&$ Astrophysics, 285.

Laing, R. A., Riley, J. M., and Longair, M. S. (1983). Bright radio sources at 178 $\mathrm{MHz}$ - Flux densities, optical identifications and the cosmological evolution of powerful radio galaxies. Mon. Not. R. Astron. Soc., 204:151-187.

Lane, W. M., Cotton, W. D., van Velzen, S., et al. (2014). The Very Large Array Low-frequency Sky Survey Redux (VLSSr). Mon. Not. R. Astron. Soc., 440:327-338.

Lauberts, A. (1982). ESO/Uppsala survey of the ESO(B) atlas. Garching: European Southern Observatory (ESO), 1982.

Ledlow, M. J., Owen, F. N., and Keel, W. C. (1998). An Unusual Radio Galaxy in Abell 428: A Large, Powerful FR I Source in a Disk-dominated Host. The Astrophysical Journal, 495:227-238.

Leir, A. A. and van den Bergh, S. (1977). A Study of 1889 Rich Clusters of Galaxies. The Astrophysical Journal Supplementary Series, 34:381-403.

Lenc, E., Gaensler, B. M., Sun, X. H., et al. (2016). Low-frequency Observations of Linearly Polarized Structures in the Interstellar Medium near the South Galactic Pole. The Astrophysical Journal, 830:38.

Lequeux, J. (1971). The Radio Continuum of Galaxies. II. The Origin of the Continuum Emission in Spiral Galaxies. Astronomy \&f Astrophysics, 15:42.

Liang, H., Ekers, R. D., Hunstead, R. W., et al. (2001). J06587-5558: a very unusual polarized radio source. Mon. Not. R. Astron. Soc., 328:L21-L26.

Liang, H., Hunstead, R. W., Birkinshaw, M., et al. (2000). A Powerful Radio Halo in the Hottest Known Cluster of Galaxies 1E 0657-56. The Astrophysical Journal, 544:686-701. 
Liu, J., Hennig, C., Desai, S., et al. (2015). Optical confirmation and redshift estimation of the Planck cluster candidates overlapping the Pan-STARRS Survey. Mon. Not. R. Astron. Soc., 449:3370-3380.

Longair, M. S. (1994). High energy astrophysics. Volume 2. Stars, the Galaxy and the interstellar medium. Cambridge University Press, Cambridge (UK), 1994.

Lotz, J., Mountain, M., Grogin, N. A., et al. (2014). The HST Frontier Fields. In American Astronomical Society Meeting Abstracts \#223, volume 223 of American Astronomical Society Meeting Abstracts, p. 254.01.

Loveday, J. (1996). The APM Bright Galaxy Catalogue. Mon. Not. R. Astron. Soc., 278:1025-1048.

Mahajan, S., Raychaudhury, S., and Pimbblet, K. A. (2012). Plunging fireworks: why do infalling galaxies light up on the outskirts of clusters? Mon. Not. R. Astron. Soc., 427:1252-1265.

Mainzer, A., Bauer, J., Grav, T., et al. (2011). Preliminary Results from NEOWISE: An Enhancement to the Wide-field Infrared Survey Explorer for Solar System Science. The Astrophysical Journal, 731:53.

Mao, M. Y., Johnston-Hollitt, M., Stevens, J. B., et al. (2009). Head-tail Galaxies: beacons of high-density regions in clusters. Mon. Not. R. Astron. Soc., 392:1070-1079.

Mao, M. Y., Owen, F., Duffin, R., et al. (2015). J1649+2635: a grand-design spiral with a large double-lobed radio source. Mon. Not. R. Astron. Soc., 446:4176-4185.

Markevitch, M. (2006). Chandra Observation of the Most Interesting Cluster in the Universe. In Wilson, A., ed., The X-ray Universe 2005, volume 604 of ESA Special Publication, p. 723.

Martin, D. C., Fanson, J., Schiminovich, D., et al. (2005). The Galaxy Evolution Explorer: A Space Ultraviolet Survey Mission. The Astrophysical Journal Letters, 619:L1-L6.

Martinet, N., Clowe, D., Durret, F., et al. (2016). Weak lensing study of 16 DAFT/FADA clusters: Substructures and filaments. Astronomy \& Astrophysics, 590:A69.

Martinez Aviles, G., Ferrari, C., Johnston-Hollitt, M., et al. (2016). ATCA observations of the MACS-Planck Radio Halo Cluster Project. I. New detection of a radio halo in PLCK G285.0-23.7. Astronomy \& Astrophysics, 595:A116.

Mauch, T., Murphy, T., Buttery, H. J., et al. (2003). SUMSS: a wide-field radio imaging survey of the southern sky - II. The source catalogue. Mon. Not. R. Astron. Soc., 342:1117-1130.

Meurer, G. R., Heckman, T. M., and Calzetti, D. (1999). Dust Absorption and the Ultraviolet Luminosity Density at z $\sim 3$ as Calibrated by Local Starburst Galaxies. The Astrophysical Journal, 521:64-80. 
Meurer, G. R., Heckman, T. M., Leitherer, C., et al. (1995). Starbursts and Star Clusters in the Ultraviolet. The Astronomical Journal, 110:2665.

Miley, G. K., Perola, G. C., van der Kruit, P. C., et al. (1972). Active Galaxies with Radio Trails in Clusters. Nature, 237:269-272.

Miniati, F., Jones, T. W., Kang, H., et al. (2001). Cosmic-Ray Electrons in Groups and Clusters of Galaxies: Primary and Secondary Populations from a Numerical Cosmological Simulation. The Astrophysical Journal, 562:233-253.

Mulcahy, D. D., Mao, M. Y., Mitsuishi, I., et al. (2016). Discovery of a lowluminosity spiral DRAGN. Astronomy \&6 Astrophysics, 595:L8.

Murgia, M., Parma, P., de Ruiter, H. R., et al. (2001). A multi-frequency study of the radio galaxy NGC 326. I. The data. Astronomy \& Astrophysics, 380:102116.

Murgia, M., Parma, P., Mack, K.-H., et al. (2011). Dying radio galaxies in clusters. Astronomy \&6 Astrophysics, 526:A148.

Murphy, T., Mauch, T., Green, A., et al. (2007). The second epoch Molonglo Galactic Plane Survey: compact source catalogue. Mon. Not. R. Astron. Soc., 382:382-392.

Nemmen, R. S., Georganopoulos, M., Guiriec, S., et al. (2012). A Universal Scaling for the Energetics of Relativistic Jets from Black Hole Systems. Science, $338: 1445$.

Oegerle, W. R. and Hill, J. M. (2001). Dynamics of cD Clusters of Galaxies. IV. Conclusion of a Survey of 25 Abell Clusters. The Astronomical Journal, 122:2858-2873.

Offringa, A. R., Trott, C. M., Hurley-Walker, N., et al. (2016). Parametrizing Epoch of Reionization foregrounds: a deep survey of low-frequency pointsource spectra with the Murchison Widefield Array. Mon. Not. R. Astron. Soc., 458:1057-1070.

Offringa, A. R., van de Gronde, J. J., and Roerdink, J. B. T. M. (2012). A morphological algorithm for improving radio-frequency interference detection. Astronomy \& Astrophysics, 539:A95.

Owen, F. N., Ledlow, M. J., and Keel, W. C. (1995). Optical spectroscopy of radio galaxies in Abell clusters. 1: Redshifts and emission-line properties. The Astronomical Journal, 109:14-25.

Pacholczyk, A. G. (1970). Radio astrophysics. Nonthermal processes in galactic and extragalactic sources. Series of Books in Astronomy and Astrophysics, San Francisco: Freeman, 1970.

Paciga, G., Albert, J. G., Bandura, K., et al. (2013). A simulation-calibrated limit on the H I power spectrum from the GMRT Epoch of Reionization experiment. Mon. Not. R. Astron. Soc., 433:639-647.

Paturel, G., Petit, C., Prugniel, P., et al. (2003). HYPERLEDA. I. Identification and designation of galaxies. Astronomy \& Astrophysics, 412:45-55. 
Pearson, D. W. and Batuski, D. J. (2013). Locating bound structure in an accelerating universe. Mon. Not. R. Astron. Soc., 436:796-806.

Peebles, P. J. E. (1980). The large-scale structure of the universe. Princeton Univ. Press, Princeton, N. J.

Petrosian, V. (2001). On the Nonthermal Emission and Acceleration of Electrons in Coma and Other Clusters of Galaxies. The Astrophysical Journal, 557:560 572 .

Petrosian, V. and East, W. E. (2008). Heating and Acceleration of Intracluster Medium Electrons by Turbulence. The Astrophysical Journal, 682:175-185.

Piffaretti, R., Arnaud, M., Pratt, G. W., et al. (2011). The MCXC: a metacatalogue of x-ray detected clusters of galaxies. Astronomy \& Astrophysics, 534:A109.

Pimbblet, K. A., Smail, I., Edge, A. C., et al. (2006). The Las Campanas/AngloAustralian Telescope Rich Cluster Survey - III. Spectroscopic studies of X-ray bright galaxy clusters at $\mathrm{z}^{\sim}$ 0.1. Mon. Not. R. Astron. Soc., 366:645-666.

Planck Collaboration, Ade, P. A. R., Aghanim, N., et al. (2014). Planck 2013 results. XXIX. The Planck catalogue of Sunyaev-Zeldovich sources. Astronomy E Astrophysics, 571:A29.

Planck Collaboration, Ade, P. A. R., Aghanim, N., et al. (2015a). Planck 2013 results. XXXII. The updated Planck catalogue of Sunyaev-Zeldovich sources. Astronomy \& Astrophysics, 581:A14.

Planck Collaboration, Ade, P. A. R., Aghanim, N., et al. (2015b). Planck intermediate results. XXVI. Optical identification and redshifts of Planck clusters with the RTT150 telescope. Astronomy \& Astrophysics, 582:A29.

Pratley, L., Johnston-Hollitt, M., Dehghan, S., et al. (2013). Using head-tail galaxies to constrain the intracluster magnetic field: an in-depth study of PKS J0334-3900. Mon. Not. R. Astron. Soc., 432:243-257.

Pratt, G. W., Croston, J. H., Arnaud, M., et al. (2009). Galaxy cluster X-ray luminosity scaling relations from a representative local sample (REXCESS). Astronomy \& Astrophysics, 498:361-378.

Prokhorov, D. A. and Churazov, E. M. (2014). Counting gamma rays in the directions of galaxy clusters. Astronomy \& Astrophysics, 567:A93.

Protheroe, R. J. (1999). Acceleration and interaction of ultra high energy cosmic rays. In Duvernois, M. A., ed., Topics in Cosmic-Ray Astrophysics, volume 230, p. 247.

Quintana, H. and Ramirez, A. (1995). Redshifts of 165 Abell and southern rich clusters of galaxies. The Astrophysical Journal Supplementary Series, 96:343358.

Randall, S. W., Clarke, T. E., Nulsen, P. E. J., et al. (2010). Radio and Deep Chandra Observations of the Disturbed Cool Core Cluster Abell 133. The Astrophysical Journal, 722:825-846. 
Ratcliffe, A., Shanks, T., Parker, Q. A., et al. (1998). The Durham/UKST Galaxy Redshift Survey - V. The catalogue. Mon. Not. R. Astron. Soc., 300:417-462.

Rees, N. (1990). A deep 38- $\mathrm{MHz}$ radio survey of the area delta greater than +60 degrees. Mon. Not. R. Astron. Soc., 244:233-246.

Reichardt, C. L., Stalder, B., Bleem, L. E., et al. (2013). Galaxy Clusters Discovered via the Sunyaev-Zel'dovich Effect in the First 720 Square Degrees of the South Pole Telescope Survey. The Astrophysical Journal, 763:127.

Reichert, G., Mason, K. O., Charles, P. A., et al. (1981). Low energy X-ray emission from five galaxy cluster sources. The Astrophysical Journal, 247:803-812.

Reynolds, J. (1994). A Revised Flux Scale for the AT Compact Array. Technical report, Australia Telescope National Facility.

Röttgering, H. J. A., Wieringa, M. H., Hunstead, R. W., et al. (1997). The extended radio emission in the luminous X-ray cluster A3667. Mon. Not. R. Astron. Soc., 290:577-584.

Rybicki, G. B. and Lightman, A. L. (2004). Radiative processes in astrophysics. WILEY-VCH Verlag GmbH \& Co. KGaA, Weinheim.

Salim, S., Rich, R. M., Charlot, S., et al. (2007). UV Star Formation Rates in the Local Universe. The Astrophysical Journal Supplementary Series, 173:267292.

Sanders, J. S., Fabian, A. C., and Smith, R. K. (2011). Constraints on turbulent velocity broadening for a sample of clusters, groups and elliptical galaxies using XMM-Newton. Mon. Not. R. Astron. Soc., 410:1797-1812.

Saripalli, L., Hunstead, R. W., Subrahmanyan, R., et al. (2005). A Complete Sample of Megaparsec-sized Double Radio Sources from the Sydney University Molonglo Sky Survey. The Astronomical Journal, 130:896-922.

Sault, R. J., Teuben, P. J., and Wright, M. C. H. (1995). A Retrospective View of MIRIAD. In Shaw, R. A., Payne, H. E., and Hayes, J. J. E., eds., Astronomical Data Analysis Software and Systems IV, volume 77 of Astronomical Society of the Pacific Conference Series, p. 433.

Scaife, A. M. M., Oozeer, N., de Gasperin, F., et al. (2015). KAT-7 detection of radio halo emission in the Triangulum Australis galaxy cluster. Mon. Not. R. Astron. Soc., 451:4021-4028.

Scheuer, P. A. G. (1974). Models of extragalactic radio sources with a continuous energy supply from a central object. Mon. Not. R. Astron. Soc., 166:513-528.

Scheuer, P. A. G. and Williams, P. J. S. (1968). Radio Spectra. Annual Review of Astronomy \& Astrophysics, 6:321.

Schilizzi, R. T. and McAdam, W. B. (1975). Observations of extended sources at $408 \mathrm{MHz}$. I - The radio structures. Memoires of the Royal Astronomical Society, 79:1-73.

Schiminovich, D., Wyder, T. K., Martin, D. C., et al. (2007). The UV-Optical Color Magnitude Diagram. II. Physical Properties and Morphological Evolution On 
and Off of a Star-forming Sequence. The Astrophysical Journal Supplementary Series, 173:315-341.

Schoenmakers, A. P., de Bruyn, A. G., Röttgering, H. J. A., et al. (2001). A new sample of giant radio galaxies from the WENSS survey. I. Sample definition, selection effects and first results. Astronomy \& Astrophysics, 374:861-870.

Schoenmakers, A. P., Mack, K.-H., de Bruyn, A. G., et al. (2000). A new sample of giant radio galaxies from the WENSS survey. II. A multi-frequency radio study of a complete sample: Properties of the radio lobes and their environment. Astronomy $\&$ Astrophysics Suppl. Series, 146:293-322.

Schwope, A., Hasinger, G., Lehmann, I., et al. (2000). The ROSAT Bright Survey: II. Catalogue of all high-galactic latitude RASS sources with PSPC countrate $\mathrm{CR}>0.2 \mathrm{~s}^{-1}$. Astronomische Nachrichten, 321:1-52.

Seyfert, C. K. (1943). Nuclear Emission in Spiral Nebulae. The Astrophysical Journal, 97:28.

Shakouri, S., Johnston-Hollitt, M., and Pratt, G. W. (2016). The ATCA REXCESS Diffuse Emission Survey (ARDES) - I. Detection of a giant radio halo and a likely radio relic. Mon. Not. R. Astron. Soc., 459:2525-2538.

Shectman, S. A., Landy, S. D., Oemler, A., et al. (1996). The Las Campanas Redshift Survey. The Astrophysical Journal, 470:172.

Shimwell, T. W., Brown, S., Feain, I. J., et al. (2014). Deep radio observations of the radio halo of the bullet cluster 1E 0657-55.8. Mon. Not. R. Astron. Soc., 440:2901-2915.

Shimwell, T. W., Markevitch, M., Brown, S., et al. (2015). Another shock for the Bullet cluster, and the source of seed electrons for radio relics. Mon. Not. R. Astron. Soc., 449:1486-1494.

Singh, V., Ishwara-Chandra, C. H., Sievers, J., et al. (2015). Discovery of rare double-lobe radio galaxies hosted in spiral galaxies. Mon. Not. R. Astron. Soc., 454:1556-1572.

Sivanandam, S., Zabludoff, A. I., Zaritsky, D., et al. (2009). The Enrichment of the Intracluster Medium. The Astrophysical Journal, 691:1787-1806.

Slee, O. B. and Reynolds, J. E. (1984). Steep-spectrum radio sources in clusters of galaxies - The southern sample. Proceedings of the Astronomical Society of Australia, 5:516-529.

Slee, O. B. and Roy, A. L. (1998). An extreme example of a radio relic in Abell 4038. Mon. Not. R. Astron. Soc., 297:L86-L92.

Slee, O. B., Roy, A. L., Murgia, M., et al. (2001). Four Extreme Relic Radio Sources in Clusters of Galaxies. The Astronomical Journal, 122:1172-1193.

Song, J., Zenteno, A., Stalder, B., et al. (2012). Redshifts, Sample Purity, and BCG Positions for the Galaxy Cluster Catalog from the First 720 Square Degrees of the South Pole Telescope Survey. The Astrophysical Journal, 761:22. 
Srinivasan, R. (2015). A Radio View of the Bullet Cluster from $100 \mathrm{MHz}$ to 9 GHz. Master's thesis, Victoria University of Wellington.

Struble, M. F. and Rood, H. J. (1999). A Compilation of Redshifts and Velocity Dispersions for ACO Clusters. The Astrophysical Journal Supplementary Series, 125:35-71.

Sunyaev, R. A. and Zeldovich, Y. B. (1970). Small-Scale Fluctuations of Relic Radiation. Astrophysics and Space Science, 7:3-19.

Tingay, S. J., Goeke, R., Bowman, J. D., et al. (2013). The Murchison Widefield Array: The Square Kilometre Array Precursor at Low Radio Frequencies. Publications of the Astron. Soc. of Australia, 30:e07.

Tribble, P. C. (1993). Radio spectral ageing in a random magnetic field. Mon. Not. R. Astron. Soc., 261:57-62.

Trümper, J. (1984). ROSAT. Physica Scripta Volume T, 7:209-215.

Ulvestad, J. S., Wilson, A. S., and Sramek, R. A. (1981). Radio structures of Seyfert galaxies. II. The Astrophysical Journal, 247:419-442.

Umetsu, K., Medezinski, E., Nonino, M., et al. (2014). CLASH: Weak-lensing Shear-and-magnification Analysis of 20 Galaxy Clusters. The Astrophysical Journal, 795:163.

Venturi, T., Giacintucci, S., Brunetti, G., et al. (2007). GMRT radio halo survey in galaxy clusters at $z=0.2-0.4$. I. The REFLEX sub-sample. Astronomy $\mathcal{E}$ Astrophysics, 463:937-947.

Venturi, T., Giacintucci, S., Dallacasa, D., et al. (2008). GMRT radio halo survey in galaxy clusters at $z=0.2-0.4$. II. The eBCS clusters and analysis of the complete sample. Astronomy \& Astrophysics, 484:327-340.

Venturi, T., Giacintucci, S., Dallacasa, D., et al. (2013). Low frequency follow up of radio haloes and relics in the GMRT Radio Halo Cluster Survey. Astronomy E6 Astrophysics, 551:A24.

Voges, W., Aschenbach, B., Boller, T., et al. (1999). The ROSAT all-sky survey bright source catalogue. Astronomy \& Astrophysics, 349:389-405.

Way, M. J., Quintana, H., and Infante, L. (1997). The Dynamics of the cD Clusters Abell 119 and Abell 133. ArXiv Astrophysics e-prints.

Wayth, R. B., Lenc, E., Bell, M. E., et al. (2015). GLEAM: The GaLactic and Extragalactic All-Sky MWA Survey. Publications of the Astron. Soc. of Australia, 32:e25.

Wen, Z. L. and Han, J. L. (2013). Substructure and dynamical state of 2092 rich clusters of galaxies derived from photometric data. Mon. Not. R. Astron. Soc., 436:275-293.

Wen, Z. L., Han, J. L., and Liu, F. S. (2010). Erratum: "Galaxy Clusters Identified from the Sloan Digital Sky Survey DR6 and their Properties" (2009, ApJS, 183, 197). The Astrophysical Journal Supplementary Series, 187:272-273. 
Wen, Z. L., Han, J. L., and Liu, F. S. (2012). A Catalog of 132,684 Clusters of Galaxies Identified from Sloan Digital Sky Survey III. The Astrophysical Journal Supplementary Series, 199:34.

Whiting, M. T. (2012). DUCHAMP: a 3D source finder for spectral-line data. Mon. Not. R. Astron. Soc., 421:3242-3256.

Williamson, R., Benson, B. A., High, F. W., et al. (2011). A Sunyaev-Zel'dovichselected Sample of the Most Massive Galaxy Clusters in the $2500 \mathrm{deg}^{2}$ South Pole Telescope Survey. The Astrophysical Journal, 738:139.

Wilson, A. S. and Colbert, E. J. M. (1995). The difference between radio-loud and radio-quiet active galaxies. The Astrophysical Journal, 438:62-71.

Wilson, W. E., Ferris, R. H., Axtens, P., et al. (2011). The Australia Telescope Compact Array Broad-band Backend: description and first results. Mon. Not. R. Astron. Soc., 416:832-856.

Wolberg, J. (2006). Data Analysis Using the Method of Least Squares: Extracting the Most Information from Experiments. Springer Berlin Heidelberg: Berlin, Heidelberg.

Wright, E. L., Eisenhardt, P. R. M., Mainzer, A. K., et al. (2010). The Wide-field Infrared Survey Explorer (WISE): Mission Description and Initial On-orbit Performance. The Astronomical Journal, 140:1868-1881.

Yan, L., Donoso, E., Tsai, C.-W., et al. (2013). Characterizing the Mid-infrared Extragalactic Sky with WISE and SDSS. The Astronomical Journal, 145:55.

Yatawatta, S., de Bruyn, A. G., Brentjens, M. A., et al. (2013). Initial deep LOFAR observations of epoch of reionization windows. I. The north celestial pole. Astronomy \& Astrophysics, 550:A136.

Zaritsky, D., Gonzalez, A. H., and Zabludoff, A. I. (2006). The Fundamental Manifold of Spheroids. The Astrophysical Journal, 638:725-738.

Zhao, H.-H., Jia, S.-M., Chen, Y., et al. (2013). A Good Mass Proxy for Galaxy Clusters with XMM-Newton. The Astrophysical Journal, 778:124. 


\section{§A. Source-finding and measuring with Witch- wood}

In what follows we describe source-finding software used during the course of this work. The software does not do anything new, and was written as a pet-project by the author of this work. We describe it here because there is no documentation for it elsewhere, and it was used in the data analysis presented in all sections. The code is hosted at https://github.com/Sunmish/Witchwood.

During the course of this work it became clear the measuring flux densities of radio sources would be a necessary thing over and over again. Source-finding software exists that will take an input image, return sources above some threshold (typically some multiple of an input rms), and calculate the integrated flux density of each source. One such example, AEGEAN does this to great effect, going as far as fitting elliptical Gaussians to disentangle blended point sources. Despite its usefulness, AEGEAN is designed first and foremost for point-source-finding, whereas for this work we require software that simply measures the flux density of an extended source, without considering it as a point source (i.e. we consider the source to be larger than the beam and often of irregular morphology). In this section, we describe a simple source-finding and measuring routine named WITCHWOOD that simply finds pixel groups above a given threshold assumed to be discrete sources, and measures their integrated flux densities as well as calculating their largest angular scales.

This does not add anything beyond what is seen in other source-finding programs, rather it simply does what we needed to it do for most ${ }^{1}$ of our sources. WITCHWOOD is written in PYTHON and makes heavy use of the communitydeveloped package, ASTROPY, as well as NUMPY. It is intended to be used from with other PYTHON scripts or from the PYTHON or IPYTHON console, hence works as a normal function. The main user-functions are measure_forest and measure_tree. The reader is encouraged to ignore the tortured metaphor. The first function performs the main source-finding on an image, returning a catalogue and an optional output FITS file that shows the detected sources and their numbers, as well as an optional annotation file for SAO's DS9 or Karma's Kvis which both simply show the source number of each source. Fig. A.1 shows example FITS image with source counts, as well as the original image with the annotation file overlaid.

The second function performs the source-finding, returning the catalogue, and then finds the closest source to input coordinates. Values are returned as PYTHON variables and optionally printed to the console in a readable format shown in Fig. A.2.

\footnotetext{
${ }^{1}$ For blended sources we use AEGEAN and assume our source can be represented as a point.
} 

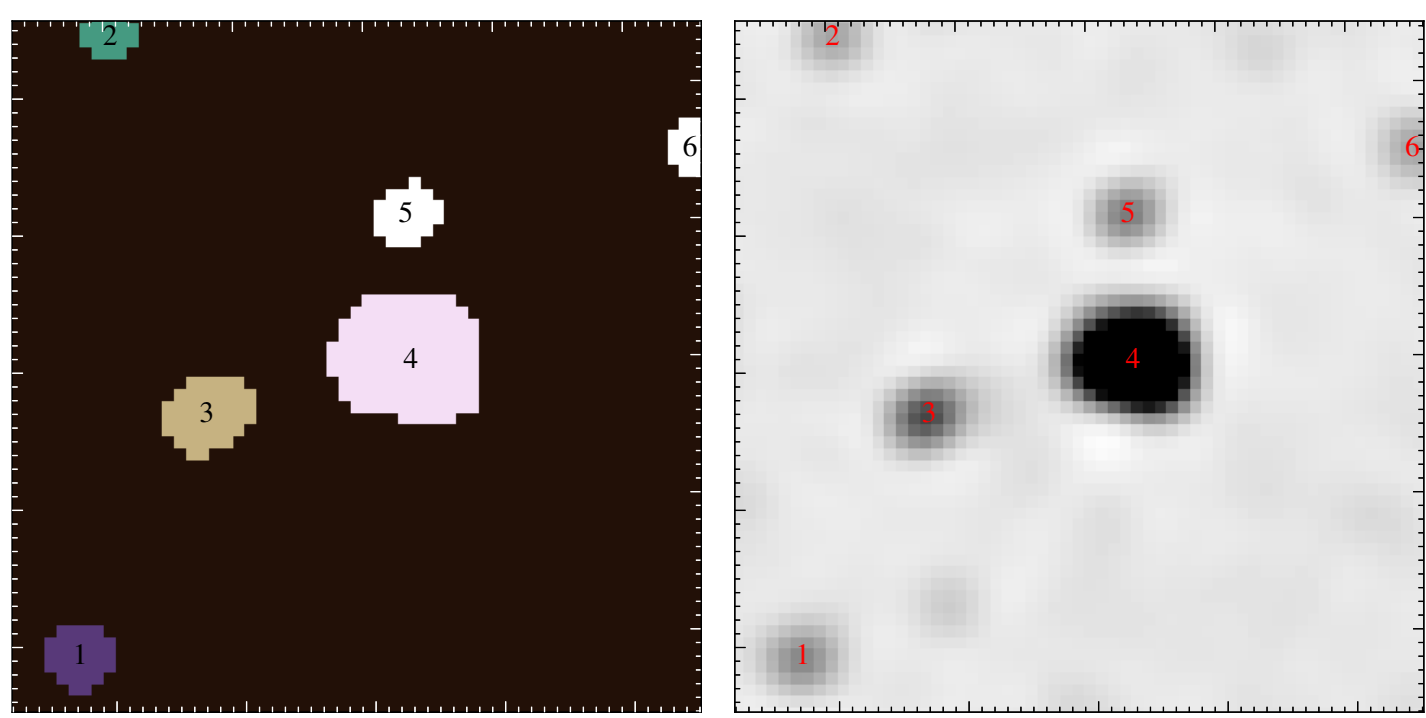

Figure A.1: Example of WITCHWOOD source-finding on an EoR0 field image of the cluster Abell 0013. Left: Sources as detected by WITCHWOOD with an rms of 12 mJy beam ${ }^{-1}$ with a cutoff of $3 \sigma_{\text {rms. }}$. The colourscale is set to the source numbers. Right: The radio image with sources marked as in the left panel.

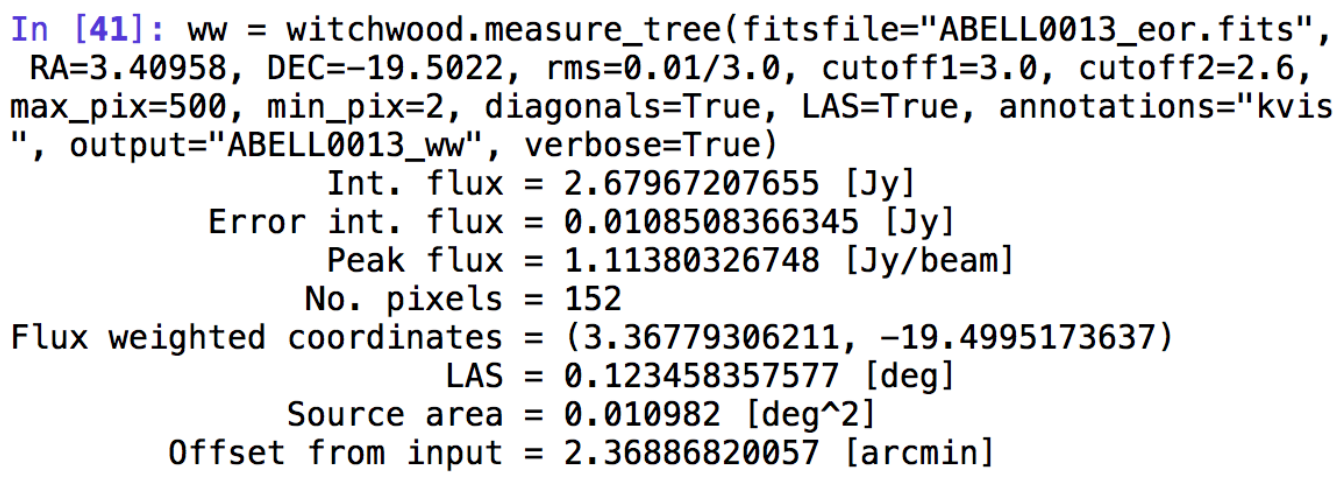

Figure A.2: Example output of WITCHWOOD during single-source-finding. The image was of Abell 0013, and the input coordinates were of the cluster centre. The nearest source is the relic slightly to the east. LAS has been optionally turned on for this measurement. Setting the output to anything but "None" will write out the example FITS file in Fig. A.1. Note that the integrated flux density has not been scaled as per §ii.1.3.2. 


\section{§.1. The floodfill algorithm}

WITCHWOOD works by making use of the floodfill algorithm. The floodfill algorithm is used in many applications (and in fact, many source-finding applications) and works by spreading from some initial point. That is, if we have a 3 by 3 array and start in the centre, we would check each surrounding cell and for any surrounding cells that meet some condition we would add that cell and do the same check with the cells surrounding that one. This continues until no border cells pass the condition. Within the context of WITCHWOOD, we make a pass of the input array and any time a pixel is detected above the input threshold, we look at the surrounding pixels to see if they too are above this threshold. If so, they are added to that source. This process can be time- and process-consuming, so we have a reference array which is simply used to check if we have looked at a pixel previously. This avoids double (and triple and quadruple, et cetera) checking. This continues over the entire input array, and sources - when found and added to - are written to dictionaries for later keeping.

\section{$\S$ A.2. Calculating the flux density and other mea- surements}

\section{$\S$ A.2.1. Flux density}

The primary purpose of WITCHWOOD was never source-finding, rather we needed an easy way of measuring flux densities of sources whose positions we already knew. The measurement of most importance is that of the integrated flux density and its associated error. WITCHWOOD is designed to work with radio images with pixel values in units of $\mathrm{Jy}_{\text {beam }}{ }^{-1}$, thus to make a measurement of the integrated flux density we simply sum the pixel values that comprise our source and correct for the beam. The floodfill algorithm defines which pixels will be used. The integrated flux density is then

$$
S_{\nu}=\sum_{n}^{N} S_{n} \times\left[\frac{\left|c_{1} c_{2}\right| 4 \ln 2}{\pi B_{\text {maj }} B_{\min }}\right] \quad[\mathrm{Jy}],
$$

where the sum is over the $N$ pixels comprising the source, $c_{1}$ and $c_{2}$ are the pixel dimenions, $B_{\text {maj }}$ and $B_{\text {min }}$ are the major and minor axes of the beam FWHM, and the factor of $\ln 2$ accounts for the integral over the Gaussian surface of the beam. The associated uncertainty is

$$
\sigma_{S_{\nu}}=\sigma_{\text {rms }} \sqrt{N_{\text {beam }}} \quad[\mathrm{Jy}],
$$

where $\sigma_{\text {rms }}$ is the user-supplied rms for the map and $N_{\text {beam }}$ is the number of beams crossing the source. We assume the rms does not vary significantly over the source. These flux density measurements are similar to those done by the software DUCHAMP (Whiting, 2012). 


\section{$\S A .2 .2$. Other quantities}

A useful secondary application of WITCHWOOD is the calculation of the largest angular scale (LAS) of any sources found. During the source-finding, any pixels that are the boundary of a detection are written to a separate dictionary tied to the associated source. During the flux density calculation step, we also compare the separations of all boundary pixels with one another. This is a reasonably long task as we need to check $N^{3 / 2}$ separations for $N$ pixels. The greatest of these is considered the LAS.

Finally, we define source positions in two ways in the same way as DUCHAMP. The first is by the brightest pixel of a source. For a point source this would be its centre, but for extended sources this is not necessarily the case. The second position is the flux-weighted coordinates. These are defined to be

$$
\begin{array}{r}
x_{w}=\frac{\sum_{n}^{N} x_{n} S_{n}}{\sum_{n}^{N} S_{n}}, \\
y_{w}=\frac{\sum_{n}^{N} y_{n} S_{n}}{\sum_{n}^{N} S_{n}},
\end{array}
$$

for pixel coordinates $x$ and $y$ which are later converted to world coordinates in degrees by use of ASTROPY. 